\title{
Copper-Catalyzed Cross-Nucleophile Coupling of $\beta$-Allenyl Silanes with Tertiary C-H Bonds: A Radical Approach to Branched 1,3-Dienes
}

Qi-Chao Shan, Lu-Min Hu, Wei Qin, and Xu-Hong Hu*

Institute of Advanced Synthesis, School of Chemistry and Molecular Engineering, Nanjing Tech University,

Nanjing 211816, China

ias_xhhu@njtech.edu.cn (X.-H. Hu)

\section{Table of Contents}

2 Optimization of the reaction conditions S3

3 Preparation of the starting materials S3

3.1 Preparation of $\beta$-allenyl silanes 1a-1q $\quad$ S3

3.2 Preparation of tertiary hydrocarbons $\mathbf{2} \mathbf{b}-\mathbf{2} \mathbf{q} \quad S 9$

$4 \quad$ The general procedure 5 (GP5) for the synthesis of $3 \quad$ S13

5 Scale-up synthesis of product 3aa $\quad$ S13

6 Derivatization reactions of product 3aa $\quad$ S14

$\begin{array}{lr}6.1 \text { Synthesis of compound } 4 & \text { S14 }\end{array}$

6.2 Synthesis of compound $5 \quad \mathrm{~S} 14$

$\begin{array}{lr}6.3 \text { Synthesis of compound } 6 & \mathbf{6} 15\end{array}$

$\begin{array}{ll}6.4 \text { Synthesis of compound } 7 & \mathbf{S 1 5}\end{array}$

$\begin{array}{lr}6.5 \text { Synthesis of compound } 8 & \text { S16 }\end{array}$

$7 \quad$ Mechanistic exploration experiments $\quad$ S16

$\begin{array}{ll}\text { 7.1 Radical trapping experiments } & \mathrm{S} 16\end{array}$

$\begin{array}{ll}7.2 \text { By-product tracking experiment } & \mathrm{S} 17\end{array}$

$8 \quad$ NMR data of the products $\quad S 18$

9 References $\quad$ S26

$10 \quad$ NMR spectra of the starting materials $\quad$ S27

11 NMR spectra of all the products $\quad$ S51 


\section{General methods}

Experiments involving moisture and/or air sensitive components were performed in oven-dried glassware by using Schlenk line techniques with a four-port dual-bank manifold under a nitrogen atmosphere. Commercial solvents and reagents were purchased from Energy-Chemical, Aladdin Bio-Chem Technology, and J\&K Scientific, and were used without further purification.

Analytical thin layer chromatography (TLC) was performed using Merck 60 F254 precoated silica gel plate $(0.2$ $\mathrm{mm}$ thickness). Subsequent to elution, plates were visualized using UV radiation (254 $\mathrm{nm}$ ) on Spectroline Model ENF-24061/F $254 \mathrm{~nm}$. Further visualization was possible by staining with basic solution of potassium permanganate or acidic solution of ceric molybdate, followed by heating on a hot plate. Flash chromatography was performed using Nuotai silica gel (200 - 300 mesh) with distilled solvents. Columns were typically packed as slurry and equilibrated with petroleum ether prior to use.

Proton nuclear magnetic resonance $\left({ }^{1} \mathrm{H} \mathrm{NMR}\right)$ and carbon nuclear magnetic resonance $\left({ }^{13} \mathrm{C} \mathrm{NMR}\right)$ spectroscopy were performed on a Bruker Advance $400 \mathrm{MHz}$ and JEOL $400 \mathrm{MHz}$ spectrometers. Chemical shifts for ${ }^{1} \mathrm{H}$ NMR spectra are reported as in units of parts per million (ppm) downfield from $\mathrm{SiMe}_{4}(\delta 0.0)$ and relative to the signal of chloroform- $d(J=7.264$, singlet). Multiplicities were given as: $s$ (singlet); $d$ (doublet); t (triplet); q (quartet); dd (doublet of doublets); ddd (doublet of doublets of doublets); dddd (doublet of doublets of doublets of doublets); dt (doublet of triplets); $m$ (multiplet), etc. The number of protons ( $n$ ) for a given resonance is indicated by $\mathrm{nH}$. Coupling constants are reported as a $J$ value in $\mathrm{Hz}$. Carbon nuclear magnetic resonance spectra $\left({ }^{13} \mathrm{C} N \mathrm{NR}\right)$ are reported as $\delta$ in units of parts per million (ppm) downfield from $\mathrm{SiMe}_{4}(\delta=0.0)$ and relative to the signal of chloroform- $d(\delta=77.00$, triplet). To clarify the complete signal assignments, "x number" indicates the multiple carbons due to the superposition of chemical shifts.

High resolution mass spectral analysis (HRMS) was performed on Water Q-TOF Premier mass spectrometer (Thermo Electron Corporation). Gas chromatography-mass spectromter (GC-MS) analysis was conducted on a Thermo Scientific DSQ II single quadrupole GC/MS instrument with Thermo Fisher Scientific GC column TG-5MS $(30 \mathrm{~m} \times 0.25 \mathrm{~mm} \times 0.25 \mu \mathrm{m})$. 


\section{Optimization of the reaction conditions}

Table S1. Effect of Oxidants on the Reaction ${ }^{a}$<smiles>C=CC(CS(C)(=O)=O)c1ccccc1</smiles>

$1 \mathrm{a}$<smiles>C=C(C(=C)C(C)(OCC)OCC)c1ccccc1</smiles>

2a
DMSO, $70^{\circ} \mathrm{C}, 12 \mathrm{~h}$

3aa

$\begin{array}{ccc}\text { entry } & \text { oxidant } & \text { yield }(\%)^{b} \\ 1 & \text { DTBP } & 44 \\ 2 & \text { TBHP } & \text { n.d. } \\ 3 & \text { TBPB } & \text { n.d. } \\ 4 & \text { BPO } & \text { n.d. } \\ 5 & \mathrm{LPO} & \text { n.d. } \\ 6 & \mathrm{Ag}_{2} \mathrm{CO}_{3} & \text { n.d. }\end{array}$

${ }^{a}$ Reaction conditions: $1 \mathrm{a}(0.15 \mathrm{mmol}), \mathbf{2 a}(0.225 \mathrm{mmol}), \mathrm{CuBr}_{2}(20 \mathrm{~mol} \%)$ and oxidant $(0.3 \mathrm{mmol})$ in $\mathrm{DMSO}(1.5 \mathrm{~mL})$ at $70{ }^{\circ} \mathrm{C}$ for $12 \mathrm{~h} .{ }^{b}$ Yield of isolated product. n.d. = not detected; TBHP = tert-butyl hydroperoxide; TBPB = tert-butyl peroxybenzoate; BPO = benzoyl peroxide; LPO = lauroyl peroxide.

Table S2. Effect of Solvents on the Reaction ${ }^{a}$

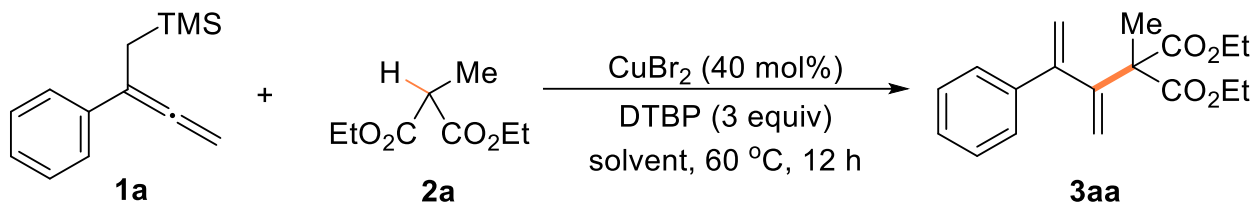

$\begin{array}{ccc}\text { entry } & \text { solvent } & \text { yield }(\%)^{b} \\ 1 & \text { DMSO } & 70 \\ 2 & \text { DMF } & \text { n.d. } \\ 3 & \mathrm{PhCl} & \text { n.d. } \\ 4 & 1,2-\mathrm{DCE} & \text { n.d. } \\ 5 & \mathrm{THF} & \text { trace } \\ 6 & \text { toluene } & \text { n.d. } \\ 7 & \mathrm{CH}_{3} \mathrm{CN} & 13\end{array}$

aReaction conditions: $1 \mathrm{a}(0.21 \mathrm{mmol}), \mathbf{2 a}(0.15 \mathrm{mmol}), \mathrm{CuBr}_{2}(40 \mathrm{~mol} \%)$ and DTBP $(0.45 \mathrm{mmol})$ in solvent $(1.5 \mathrm{~mL})$ at $60{ }^{\circ} \mathrm{C}$ for $12 \mathrm{~h} .{ }^{b}$ Yield of isolated product. n.d. $=$ not detected. 1,2-DCE $=1,2$-dichloroethane.

\section{Preparation of the starting materials}

\subsection{Preparation of $\beta$-allenyl silanes 1a-1q}


<smiles>C=C=C(CS)c1ccccc1</smiles>

$1 a$<smiles>C=C=C(CS(C)(=O)=O)c1ccc(C)cc1</smiles>

1b<smiles>C=C=C(CS(C)(=O)=O)c1ccc(C(C)(C)C)cc1</smiles>

$1 c$<smiles>C=C=C(CS)c1cccc(OC)c1</smiles>

1d<smiles>C=C=C(CS)c1ccc(Cl)cc1</smiles>

$1 e$<smiles>C=C=C(CS)c1ccc(C#N)cc1</smiles>

$1 f$<smiles>C=C=C(CS(C)(=O)=O)c1ccc(C(F)(F)F)cc1</smiles>

$1 \mathrm{~g}$<smiles>C=C=C(CS)c1ccc([N+](=O)[O-])cc1</smiles>

1h<smiles>C=C=C(CS(C)(=O)=O)c1ccc2ccccc2c1</smiles>

$1 \mathrm{~m}$<smiles>C=C=C(CS)c1ccc(C(=O)OCC)cc1</smiles>

$1 \mathrm{i}$<smiles>C=C=C(CS)c1ccsc1</smiles>

1n<smiles>C=C=C(CS)c1cccc(Br)c1</smiles>

$1 \mathrm{j}$<smiles>C=C=C(CS)c1ccccc1F</smiles>

$1 k$<smiles>C=C=C(CS(C)(=O)=O)c1ccccc1C</smiles>

$1 I$<smiles>CS(=O)(=O)CC(=CCCc1ccccc1)c1ccccc1</smiles>

$1 \mathrm{q}$<smiles>CCC(C)C=C=C(CS(C)(=O)=O)c1ccccc1</smiles>

$1 p$<smiles>CCCC=C=C(CS(C)(=O)=O)c1ccccc1</smiles>

10

3.1.1 Compounds $1 \mathrm{a}-1 \mathrm{~m}$ were prepared according to the general procedure 1 (GP1) ${ }^{[1]}$

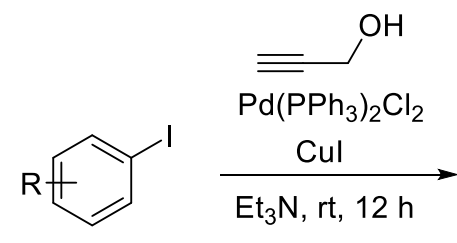<smiles>OCC#Cc1cc[R]cc1</smiles>

S1

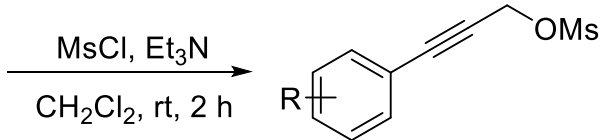

S2

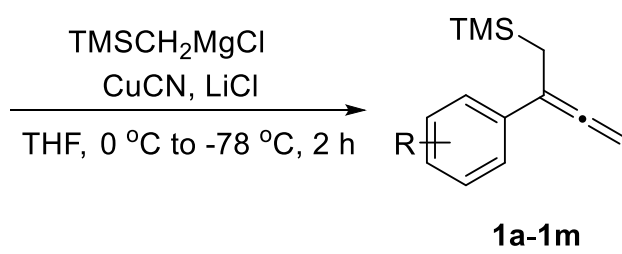

(1) To a solution of aryl iodide (5 mmol, 1.0 equiv) and propargyl alcohol (840.5 mg, $15 \mathrm{mmol}, 3.0$ equiv) in triethylamine (25 mL, $0.2 \mathrm{M})$ was added $\mathrm{Pd}\left(\mathrm{PPh}_{3}\right)_{2} \mathrm{Cl}_{2}(35.1 \mathrm{mg}, 0.05 \mathrm{mmol}, 1 \mathrm{~mol} \%)$ and Cul $(47.6 \mathrm{mg}, 0.25 \mathrm{mmol}$, $5 \mathrm{~mol} \%$ ) under $\mathrm{N}_{2}$ atmosphere. The resulting mixture was stirred at ambient temperature for $12 \mathrm{~h}$. After completion of the reaction as monitored by TLC, the reaction mixture was diluted with EtOAc $(20 \mathrm{~mL})$ and filtered through a short pad of Celite ${ }^{\circledR}$. The filtrate was concentrated in vacuo and the crude residue were purified by flash column chromatography (petroleum ether/EtOAc) on silica gel to afford the propargylic alcohol S1.

(2) To a solution of the individual propargylic alcohol $\mathbf{S 1}$ (1.0 equiv) and triethylamine (2.0 equiv) in $\mathrm{CH}_{2} \mathrm{Cl}_{2}$ $(0.2 \mathrm{M})$ was added methanesulfonyl chloride (1.5 equiv) dropwise at $0{ }^{\circ} \mathrm{C}$ under $\mathrm{N}_{2}$ atmosphere. The reaction 
mixture was allowed to warm to room temperature. After full conversion, saturated aqueous $\mathrm{NaHCO}_{3}(10 \mathrm{~mL})$ was added to quench the reaction and the aqueous layer was extracted with $\mathrm{CH}_{2} \mathrm{Cl}_{2}(15 \mathrm{~mL} \times 3)$. The combined organic extracts were washed with $\mathrm{H}_{2} \mathrm{O}(20 \mathrm{~mL})$ and brine $(10 \mathrm{~mL})$, dried over $\mathrm{Na}_{2} \mathrm{SO}_{4}$ and concentrated in vacuo. The crude products were purified by flash column chromatography (petroleum ether/EtOAc) to afford $\mathbf{S 2}$.

(3) To an ice-cooled suspension of $\mathrm{CuCN}$ (3.0 equiv) and $\mathrm{LiCl}$ (6.0 equiv) in anhydrous THF ( $0.1 \mathrm{M})$ was added a solution of $\mathrm{Me}_{3} \mathrm{SiCH}_{2} \mathrm{MgCl}$ (1.0 M in $\mathrm{Et}_{2} \mathrm{O}, 2.5$ equiv). After being stirred for $25 \mathrm{~min}$, the mixture was cooled to $78^{\circ} \mathrm{C}$ and a solution of $\mathbf{S 2}(1.0$ equiv) in THF $(5 \mathrm{~mL})$ was added dropwise. After $2 \mathrm{~h}$, the reaction was quenched with saturated aqueous $\mathrm{NH}_{4} \mathrm{Cl}(5 \mathrm{~mL})$. The reaction mixture was extracted with $\mathrm{Et}_{2} \mathrm{O}(20 \mathrm{~mL} \times 3)$. The combined organic layers were washed with brine $(10 \mathrm{~mL})$, dried over $\mathrm{Na}_{2} \mathrm{SO}_{4}$ and concentrated in vacuo. The crude products were purified by flash column chromatography (petroleum ether/EtOAc) to afford $\beta$-allenyl silanes $\mathbf{1 a - 1 m}$.

\subsubsection{Compounds 10-1q were prepared according to the general procedure 2 (GP2) ${ }^{[1]}$}

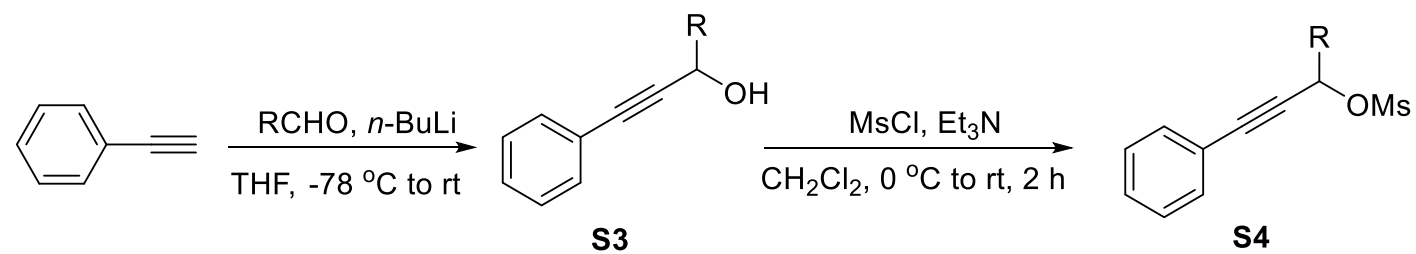<smiles>[R]C=C(CS(C)(=O)=O)c1ccccc1</smiles>

(1) To a $100 \mathrm{~mL}$ round bottom flask equipped with a stir bar was added phenylacetylene $(1.02 \mathrm{~g}, 10 \mathrm{mmol}, 1.0$ equiv) and THF (20 mL) and then cooled to $-78^{\circ} \mathrm{C}$. $n$-BuLi (2.5 M in hexane, $4.0 \mathrm{~mL}, 1.0$ equiv) was added dropwise and the slightly yellow solution was stirred for $30 \mathrm{~min}$. The corresponding aldehyde (10.0 mmol, 1.0 equiv) was added and the mixture was allowed to warm to room temperature and further stirred for $1 \mathrm{~h}$. After completion, the reaction was quenched with aqueous $\mathrm{HCl}(1.0 \mathrm{M})$ and then extracted with $\mathrm{CH}_{2} \mathrm{Cl}_{2}(20 \mathrm{~mL} \times 3)$. The combined organic layers were washed with brine $(10 \mathrm{~mL})$, dried over $\mathrm{Na}_{2} \mathrm{SO}_{4}$, and concentrated in vacuo. The crude product was purified by flash column chromatography (petroleum ether/EtOAc) to provide the corresponding propargylic alcohol S3.

(2) To a solution of the individual propargylic alcohol $\mathbf{S 3}$ (1.0 equiv) and triethylamine (2.0 equiv) in $\mathrm{CH}_{2} \mathrm{Cl}_{2}$ $(0.2 \mathrm{M})$ was added methanesulfonyl chloride (1.5 equiv) dropwise at $0{ }^{\circ} \mathrm{C}$ under $\mathrm{N}_{2}$ atmosphere. The reaction mixture was allowed to warm to room temperature and stirred for $2 \mathrm{~h}$. After completion, saturated aqueous $\mathrm{NaHCO}_{3}$ $(15 \mathrm{~mL})$ was added and the aqueous layer was extracted with $\mathrm{CH}_{2} \mathrm{Cl}_{2}(15 \mathrm{~mL} \times 3)$. The combined organic extracts were washed with $\mathrm{H}_{2} \mathrm{O}(20 \mathrm{~mL})$ and brine $(10 \mathrm{~mL})$, dried over $\mathrm{Na}_{2} \mathrm{SO}_{4}$ and concentrated in vacuo. The crude product was purified by flash column chromatography (petroleum ether/EtOAc) to afford S4.

(3) To an ice-cooled suspension of $\mathrm{CuCN}$ (3.0 equiv) and $\mathrm{LiCl}$ (6.0 equiv) in anhydrous THF ( $0.1 \mathrm{M})$ was added 
a solution of $\mathrm{Me}_{3} \mathrm{SiCH}_{2} \mathrm{MgCl}$ (1.0 M in $\mathrm{Et}_{2} \mathrm{O}, 2.5$ equiv). After being stirred for 25 min, the mixture was cooled to $78{ }^{\circ} \mathrm{C}$ and a solution of the $\mathbf{S 4}$ (1.0 equiv) in THF $(5 \mathrm{~mL})$ was added. After $2 \mathrm{~h}$, the reaction was quenched with saturated aqueous $\mathrm{NH}_{4} \mathrm{Cl}(5 \mathrm{~mL})$. The reaction mixture was extracted with $\mathrm{Et}_{2} \mathrm{O}(20 \mathrm{~mL} \times 3)$. The combined organic layers were washed with brine $(10 \mathrm{~mL})$, dried over $\mathrm{Na}_{2} \mathrm{SO}_{4}$ and concentrated in vacuo. The crude products were purified by flash column chromatography (petroleum ether/EtOAc) to afford $\beta$-allenyl silanes 10-1q.

\section{Trimethyl(2-phenylbuta-2,3-dien-1-yl)silane (1a)}<smiles>C=C=C(CS(C)(=O)=O)c1ccccc1</smiles>

The title compound was prepared according to GP1 and isolated as a colorless oil $(647.8 \mathrm{mg}$, $3.20 \mathrm{mmol}, 64 \%) .{ }^{1} \mathrm{H}$ NMR (400 MHz, Chloroform- $\left.d\right) \delta 7.42-7.37(\mathrm{~m}, 2 \mathrm{H}), 7.30-7.26(\mathrm{~m}, 3 \mathrm{H})$, 4.94 (t, $J=2.5 \mathrm{~Hz}, 2 \mathrm{H}), 1.57$ (t, $J=2.5 \mathrm{~Hz} .2 \mathrm{H}), 0.12(\mathrm{~s}, 9 \mathrm{H})$. The spectral data matched those reported previously. ${ }^{[1]}$

\section{Trimethyl(2-(p-tolyl)buta-2,3-dien-1-yl)silane (1b)}<smiles>C=C=C(CS)c1ccc(C)cc1</smiles>

The title compound was prepared according to GP1 and isolated as a colorless oil (616.7 mg, $2.85 \mathrm{mmol}, 57 \%) .{ }^{1} \mathrm{H}$ NMR (400 MHz, Chloroform- $d$ ) $\delta 7.30$ (d, $\left.J=8.2 \mathrm{~Hz}, 2 \mathrm{H}\right), 7.12$ (d, $J=8.0 \mathrm{~Hz}, 2 \mathrm{H}), 5.01(\mathrm{t}, J=2.6 \mathrm{~Hz}, 2 \mathrm{H}), 2.34(\mathrm{~s}, 3 \mathrm{H}), 1.79(\mathrm{t}, J=2.6 \mathrm{~Hz}, 2 \mathrm{H}), 0.02(\mathrm{~s}, 9 \mathrm{H})$. ${ }^{13} \mathrm{C}$ NMR $(101 \mathrm{MHz}$, Chloroform-d) $\delta 209.0,136.0,134.3,128.9 \times 2,126.0 \times 2,102.1,77.1$, 21.0, 18.5, -1.0 × 3. HRMS (ESI) m/z: $[\mathrm{M}+\mathrm{H}]^{+}$Calcd for $\mathrm{C}_{14} \mathrm{H}_{21} \mathrm{Si} 217.1407$; Found: 217.1413

\section{(2-(4-(tert-Butyl)phenyl)buta-2,3-dien-1-yl)trimethylsilane (1c)}<smiles>C=C(CS(C)(=O)=O)c1ccc(C(C)(C)C)cc1</smiles>

The title compound was prepared according to GP1 and isolated as a colorless oil $(775.4$ mg, $3.00 \mathrm{mmol}, 60 \%) .{ }^{1} \mathrm{H}$ NMR (400 MHz, Chloroform- $d$ ) $\delta 7.25$ (s, 4H), $4.92(\mathrm{t}, J=2.7 \mathrm{~Hz}$, $2 \mathrm{H}), 1.70(\mathrm{t}, J=2.6 \mathrm{~Hz}, 2 \mathrm{H}), 1.23(\mathrm{~s}, 9 \mathrm{H}),-0.06(\mathrm{~s}, 9 \mathrm{H}) .{ }^{13} \mathrm{C} \mathrm{NMR}(101 \mathrm{MHz}$, Chloroformd) $\delta 209.2,149.3,134.4,125.8 \times 2,125.1 \times 2,102.0,77.2,34.4,31.3 \times 3,18.4,-1.0 \times 3$. HRMS (ESI) m/z: [M + H] $]^{+}$Calcd for $\mathrm{C}_{17} \mathrm{H}_{27} \mathrm{Si} 259.1877$; Found: 259.1882.

\section{(2-(3-Methoxyphenyl)buta-2,3-dien-1-yl)trimethylsilane (1d)}

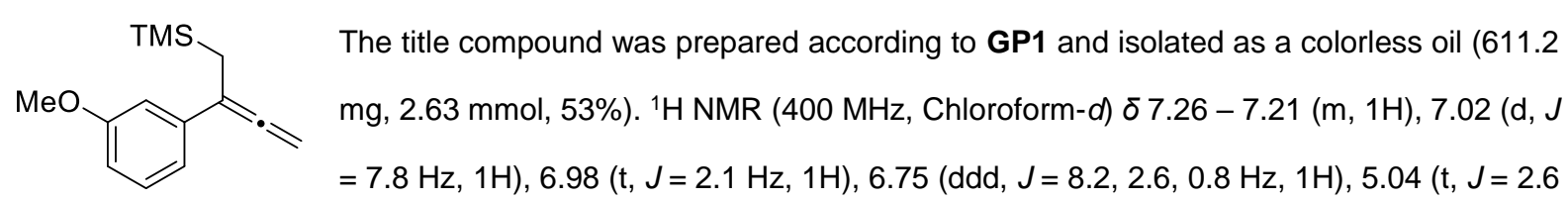
$\mathrm{Hz}, 2 \mathrm{H}), 3.82(\mathrm{~s}, 3 \mathrm{H}), 1.80(\mathrm{t}, J=2.6 \mathrm{~Hz}, 2 \mathrm{H}), 0.04(\mathrm{~s}, 9 \mathrm{H}) .{ }^{13} \mathrm{C} \mathrm{NMR}(101 \mathrm{MHz}$, Chloroform-d) $\delta 209.2,159.4$, 138.9, 129.0, 118.7, 112.0, 111.7, 102.2, 77.3, 55.1, 18.5, $-1.1 \times 3 . \mathrm{HRMS}(\mathrm{ESI}) \mathrm{m} / \mathrm{z}:[\mathrm{M}+\mathrm{H}]^{+}$Calcd for $\mathrm{C}_{14} \mathrm{H}_{21} \mathrm{OSi}$ 233.1356; Found: 233.1362 . 
<smiles>C=C=C(CS)c1ccc(Cl)cc1</smiles>

The title compound was prepared according to GP1 and isolated as a colorless oil (698.6 mg, $2.95 \mathrm{mmol}, 59 \%) .{ }^{1} \mathrm{H}$ NMR (400 MHz, Chloroform- $\left.d\right) \delta 7.35-7.30$ (m, 2H), 7.27 (q, $J=$ $1.1 \mathrm{~Hz}, 1 \mathrm{H}), 7.26-7.25(\mathrm{~m}, 1 \mathrm{H}), 5.03(\mathrm{t}, J=2.6 \mathrm{~Hz}, 2 \mathrm{H}), 1.76(\mathrm{t}, J=2.6 \mathrm{~Hz}, 2 \mathrm{H}), 0.01(\mathrm{~s}$, $9 H) .{ }^{13} \mathrm{C}$ NMR (101 MHz, Chloroform-d) $\delta 209.2,136.0,132.1,128.4 \times 2,127.5 \times 2,101.7$, 77.9, 18.5, $-0.9 \times 3$. HRMS (ESI) m/z: [M + H] Calcd for $\mathrm{C}_{13} \mathrm{H}_{18} \mathrm{ClSi} 237.0861$; Found: 237.0866.

\section{4-(1-(Trimethylsilyl)buta-2,3-dien-2-yl)benzonitrile (1f)}<smiles>C=C=C(CS)c1ccc(C#N)cc1</smiles>

The title compound was prepared according to GP1 and isolated as a colorless oil (534.3 mg, $2.35 \mathrm{mmol}, 47 \%) .{ }^{1} \mathrm{H}$ NMR (400 MHz, Chloroform- $d$ ) $\delta 7.58$ (d, $\left.J=8.5 \mathrm{~Hz}, 2 \mathrm{H}\right), 7.49$ (d, $J=8.5 \mathrm{~Hz}, 2 \mathrm{H}), 5.12(\mathrm{t}, J=2.6 \mathrm{~Hz}, 2 \mathrm{H}), 1.79(\mathrm{t}, J=2.7 \mathrm{~Hz}, 2 \mathrm{H}), 0.02(\mathrm{~s}, 9 \mathrm{H}) .{ }^{13} \mathrm{C}$ NMR $(101$ $\mathrm{MHz}$, Chloroform- $d) \delta 210.1,142.7,132.0 \times 2,126.7 \times 2,119.3,109.7,102.0,78.5,18.2,-$ $1.0 \times 3$. HRMS $(E S I) \mathrm{m} / \mathrm{z}:[M+H]^{+}$Calcd for $\mathrm{C}_{14} \mathrm{H}_{18} \mathrm{NSi} 228.1203$; Found: 228.1208 .

\section{Trimethyl(2-(4-(trifluoromethyl)phenyl)buta-2,3-dien-1-yl)silane (1g)}<smiles>C=CC(CS(C)(=O)=O)c1ccc(C(F)(F)F)cc1</smiles>
The title compound was prepared according to GP1 and isolated as a colorless oil (567.8 mg, $2.10 \mathrm{mmol}, 42 \%) .{ }^{1} \mathrm{H}$ NMR (400 MHz, Chloroform- $\left.d\right) \delta 7.57-7.45(\mathrm{~m}, 4 \mathrm{H}), 5.07(\mathrm{t}, J$ $=2.4 \mathrm{~Hz}, 2 \mathrm{H}), 1.79(\mathrm{t}, J=2.6 \mathrm{~Hz}, 2 \mathrm{H}), 0.00(\mathrm{~s}, 9 \mathrm{H}) .{ }^{13} \mathrm{C} \mathrm{NMR}(101 \mathrm{MHz}$, Chloroform- $d) \delta$ 209.7, 141.3, 128.3 (q, $J=32.3 \mathrm{~Hz}), 126.3 \times 2,125.0 \times 2$ (q, $J=3.9 \mathrm{~Hz}), 124.3$ (q, $J=$ $271.8 \mathrm{~Hz}), 101.8,77.9,18.3,-1.1 \times 3 .{ }^{19} \mathrm{~F}$ NMR $\left(377 \mathrm{MHz}\right.$, Chloroform- $d$ ) $\delta-62.18 . \mathrm{HRMS}(\mathrm{ESI}) \mathrm{m} / \mathrm{z}:[\mathrm{M}+\mathrm{H}]^{+}$Calcd for $\mathrm{C}_{14} \mathrm{H}_{18} \mathrm{~F}_{3} \mathrm{Si} 271.1124$; Found: 271.1130 .

\section{Trimethyl(2-(4-nitrophenyl)buta-2,3-dien-1-yl)silane (1h)}<smiles>C=C(CS(=O)(=O)[O-])c1ccc([N+](=O)[O-])cc1</smiles>

The title compound was prepared according to GP1 and isolated as a colorless oil (494.7 $\mathrm{mg}, 2.00 \mathrm{mmol}, 40 \%) .{ }^{1} \mathrm{H}$ NMR (400 MHz, Chloroform- $\left.d\right) \delta 8.15(\mathrm{~d}, J=9.0 \mathrm{~Hz}, 2 \mathrm{H}), 7.54$ (d, $J=8.9 \mathrm{~Hz}, 2 \mathrm{H}), 5.14(\mathrm{t}, J=2.6 \mathrm{~Hz}, 2 \mathrm{H}), 1.82(\mathrm{t}, J=2.6 \mathrm{~Hz}, 2 \mathrm{H}), 0.03(\mathrm{~s}, 9 \mathrm{H}) \cdot{ }^{13} \mathrm{C} \mathrm{NMR}$ $(101 \mathrm{MHz}$, Chloroform-d) $\delta 210.3,146.0,144.7,126.6 \times 2,123.4 \times 2,101.8,78.4,18.2,-$ $1.1 \times 3 . \mathrm{HRMS}(\mathrm{ESI}) \mathrm{m} / \mathrm{z}:[\mathrm{M}+\mathrm{H}]^{+}$Calcd for $\mathrm{C}_{13} \mathrm{H}_{18} \mathrm{NO}_{2} \mathrm{Si} 248.1101$; Found: 248.1107 .

\section{Ethyl 4-(1-(trimethylsilyl)buta-2,3-dien-2-yl)benzoate (1i)}

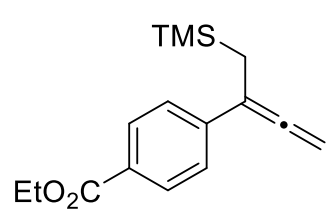

The title compound was prepared according to GP1 and isolated as a colorless oil (823.3 $\mathrm{mg}, 3.00 \mathrm{mmol}, 60 \%) .{ }^{1} \mathrm{H} \mathrm{NMR}(400 \mathrm{MHz}$, Chloroform- $d$ ) $\delta 7.97(\mathrm{~d}, J=8.6 \mathrm{~Hz}, 2 \mathrm{H}), 7.46$ (d, $J=8.5 \mathrm{~Hz}, 2 \mathrm{H}), 5.08(\mathrm{t}, J=2.6 \mathrm{~Hz}, 2 \mathrm{H}), 4.36(\mathrm{q}, J=7.2 \mathrm{~Hz}, 2 \mathrm{H}), 1.81$ (t, $J=2.6 \mathrm{~Hz}$, $2 \mathrm{H}), 1.39(\mathrm{t}, J=7.2 \mathrm{~Hz}, 3 \mathrm{H}), 0.01$ (s, 9H). ${ }^{13} \mathrm{C}$ NMR (101 MHz, Chloroform- $d$ ) $\delta 209.8$, 166.5, 142.2, $129.4 \times 2,128.2,125.9 \times 2,102.1,77.7,60.8,18.2,14.3,-1.1 \times 3 . \mathrm{HRMS}(\mathrm{ESI}) \mathrm{m} / \mathrm{z}:[\mathrm{M}+\mathrm{H}]^{+}$Calcd for $\mathrm{C}_{16} \mathrm{H}_{23} \mathrm{O}_{2} \mathrm{Si}$ 275.1462; Found: 275.1467. 


\section{(2-(3-Bromophenyl)buta-2,3-dien-1-yl)trimethylsilane (1j)}

The title compound was prepared according to GP1 and isolated as a colorless oil (787.6
$\mathrm{mg}, 2.80 \mathrm{mmol}, 56 \%) .{ }^{1} \mathrm{H} \mathrm{NMR}(400 \mathrm{MHz}, \mathrm{Chloroform}-\mathrm{d}) \delta 7.55(\mathrm{t}, \mathrm{J}=2.0 \mathrm{~Hz}, 1 \mathrm{H}), 7.31$ (ddt, 2H), 0.02 (s, 9H). ${ }^{13} \mathrm{C}$ NMR (101 MHz, Chloroform- $\left.d\right) \delta 209.2,139.8,129.6,129.3,129.1,124.6,122.4,101.5,77.9$, 18.3, $-1.1 \times 3$. HRMS $(E S I) \mathrm{m} / \mathrm{z}:[\mathrm{M}+\mathrm{H}]^{+}$Calcd for $\mathrm{C}_{13} \mathrm{H}_{18}{ }^{79} \mathrm{BrSi} 281.0356$; Found: 281.0361 .

\section{(2-(2-Fluorophenyl)buta-2,3-dien-1-yl)trimethylsilane (1k)}<smiles>C=C=C(CS)c1ccccc1F</smiles>

The title compound was prepared according to GP1 and isolated as a colorless oil $(517.8 \mathrm{mg}$, $2.35 \mathrm{mmol}, 47 \%) .{ }^{1} \mathrm{H}$ NMR (400 MHz, Chloroform- $d$ ) $\delta 7.31-7.29(\mathrm{~m}, 1 \mathrm{H}), 7.19$ (dddd, $J=8.1$, 7.0, 5.0, $1.8 \mathrm{~Hz}, 1 \mathrm{H}), 7.08(\mathrm{td}, J=7.5,1.3 \mathrm{~Hz}, 1 \mathrm{H}), 7.01$ (ddd, $J=11.1,8.1,1.3 \mathrm{~Hz}, 1 \mathrm{H}), 4.88(\mathrm{t}$, $J=2.6 \mathrm{~Hz}, 2 \mathrm{H}), 1.84(\mathrm{td}, J=2.6,0.9 \mathrm{~Hz}, 2 \mathrm{H}),-0.04(\mathrm{~s}, 9 \mathrm{H}) .{ }^{13} \mathrm{C} \mathrm{NMR}(101 \mathrm{MHz}$, Chloroform- $d)$ $\delta 209.4$ (d, $J=1.4 \mathrm{~Hz}$ ), 160.0 (d, $J=248.4 \mathrm{~Hz}$ ), 129.7 (d, $J=3.9 \mathrm{~Hz}), 128.3$ (d, $J=8.3 \mathrm{~Hz}), 126.5$ (d, $J=12.5 \mathrm{~Hz}$ ), $123.8(\mathrm{~d}, J=3.8 \mathrm{~Hz}), 115.9(\mathrm{~d}, J=22.7 \mathrm{~Hz}), 97.8,75.1(\mathrm{~d}, J=1.5 \mathrm{~Hz}), 21.0(\mathrm{~d}, J=2.9 \mathrm{~Hz}),-1.3 \times 3 .{ }^{19} \mathrm{~F} \mathrm{NMR}(377$ $\mathrm{MHz}$, Chloroform- $d$ ) $\delta$-112.96. HRMS (ESI) m/z: [M+H]+ Calcd for $\mathrm{C}_{13} \mathrm{H}_{18} \mathrm{FSi} 221.1156$; Found: 221.1161.

\section{Trimethyl(2-(o-tolyl)buta-2,3-dien-1-yl)silane (1I)}<smiles>C=C=C(CS)c1ccccc1C</smiles>

The title compound was prepared according to GP1 and isolated as a colorless oil $(586.4 \mathrm{mg}$, $2.71 \mathrm{mmol}, 54 \%) .{ }^{1} \mathrm{H}$ NMR (400 MHz, Chloroform- $\left.d\right) \delta 7.28-7.23(\mathrm{~m}, 1 \mathrm{H}), 7.21-7.13(\mathrm{~m}, 3 \mathrm{H})$, $4.78(\mathrm{t}, J=2.7 \mathrm{~Hz}, 2 \mathrm{H}), 2.40(\mathrm{~s}, 3 \mathrm{H}), 1.78(\mathrm{t}, J=2.7 \mathrm{~Hz}, 2 \mathrm{H}),-0.00(\mathrm{~s}, 9 \mathrm{H}) .{ }^{13} \mathrm{C} \mathrm{NMR}(101 \mathrm{MHz}$, Chloroform- $d$ ) $\delta 207.5,138.6,135.5,130.5,127.9,126.7,125.7,101.1,74.6,23.0,20.5,-1.2 \times$ 3. HRMS (ESI) m/z: $[\mathrm{M}+\mathrm{H}]^{+}$Calcd for $\mathrm{C}_{14} \mathrm{H}_{21} \mathrm{Si} 217.1407$; Found: 217.1413 .

\section{Trimethyl(2-(naphthalen-2-yl)buta-2,3-dien-1-yl)silane (1m)}

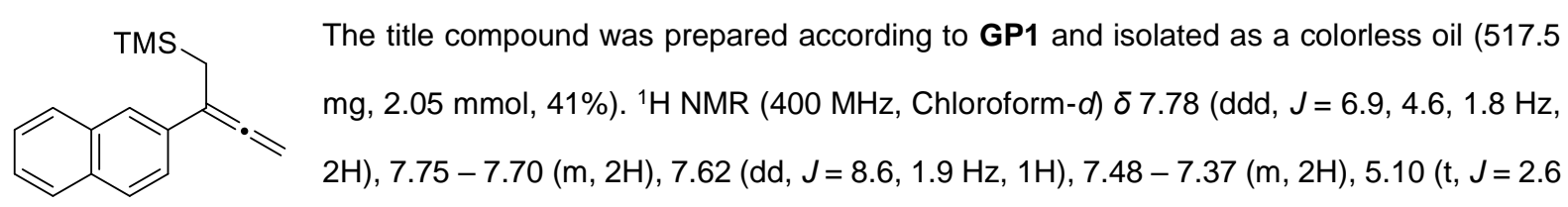
$\mathrm{Hz}, 2 \mathrm{H}), 1.92$ (t, $J=2.6 \mathrm{~Hz}, 2 \mathrm{H}), 0.04(\mathrm{~s}, 9 \mathrm{H}) .{ }^{13} \mathrm{C}$ NMR (101 MHz, Chloroform-d) $\delta$ 209.9, 134.5, 133.4, 132.2, 127.9, 127.5, 127.5, 126.0, 125.5, 125.3, 123.9, 102.6, 77.7, 18.4, -1.0 × 3. HRMS (ESI) m/z: [M + H]+ Calcd for $\mathrm{C}_{17} \mathrm{H}_{21} \mathrm{Si} 253.1407$; Found: 253.1413.

\section{Trimethyl(2-(thiophen-3-yl)buta-2,3-dien-1-yl)silane (1n)}

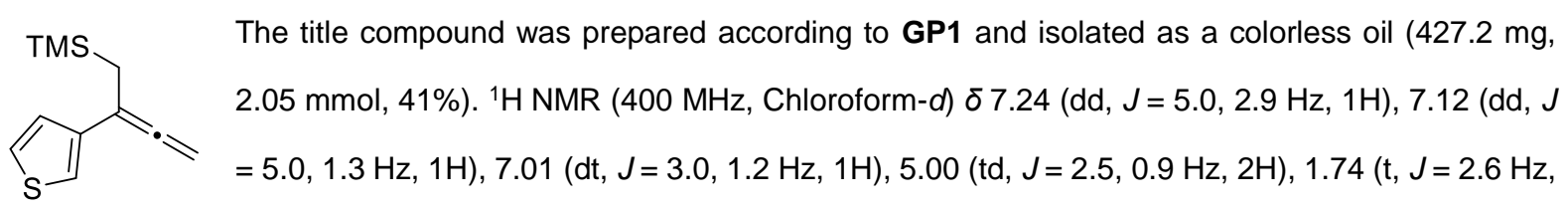


2H), $0.03(\mathrm{~s}, 9 \mathrm{H}) .{ }^{13} \mathrm{C}$ NMR (101 MHz, Chloroform-d) $\delta 209.5,139.3,126.9,125.1,119.2,98.6,77.1,19.3,-1.1 \times$ 3. HRMS (ESI) m/z: [M + H] Calcd for $\mathrm{C}_{11} \mathrm{H}_{17} \mathrm{SSi} 209.0815$; Found: 209.0820 .

\section{Trimethyl(2-phenylhepta-2,3-dien-1-yl)silane (10)}

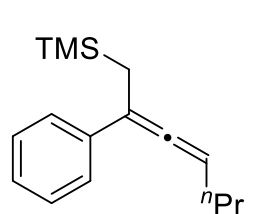

The title compound was prepared according to GP2 and isolated as a colorless oil $(974.5 \mathrm{mg}$, $3.99 \mathrm{mmol}, 40 \%) .{ }^{1} \mathrm{H}$ NMR (400 MHz, Chloroform- $d$ ) $\delta 7.40$ (dd, J = 8.5, $1.4 \mathrm{~Hz}, 2 \mathrm{H}$ ), 7.29 (dd, $J=8.5,7.0 \mathrm{~Hz}, 2 \mathrm{H}), 7.20-7.14(\mathrm{~m}, 1 \mathrm{H}), 5.41(\mathrm{tt}, J=6.9,2.4 \mathrm{~Hz}, 1 \mathrm{H}), 2.09(\mathrm{dt}, J=7.7$, $6.9 \mathrm{~Hz}, 2 \mathrm{H}), 1.80$ (dd, $J=4.6,2.4 \mathrm{~Hz}, 2 \mathrm{H}), 1.51$ (h, $J=7.4 \mathrm{~Hz}, 2 \mathrm{H}), 0.97(\mathrm{t}, J=7.3 \mathrm{~Hz}, 3 \mathrm{H})$, 0.01 (s, 9H). ${ }^{13} \mathrm{C}$ NMR (101 MHz, Chloroform-d) $\delta 204.2,138.5,128.0,126.1 \times 2,126.1 \times 2,102.4,93.1,31.6$, 22.7, 19.0, 13.9, -1.1 × 3. HRMS (ESI) m/z: $[M+H]^{+}$Calcd for $\mathrm{C}_{16} \mathrm{H}_{25} \mathrm{Si}$ 245.1720; Found: 245.1725 .

\section{Trimethyl(2-phenylocta-2,3-dien-1-yl)silane (1p)}

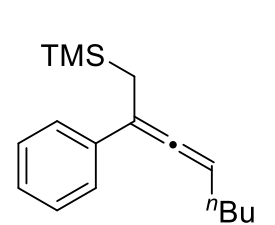

The title compound was prepared according to GP2 and isolated as a colorless oil $(784.7 \mathrm{mg}$, $3.04 \mathrm{mmol}, 30 \%) .{ }^{1} \mathrm{H}$ NMR (400 MHz, Chloroform- $d$ ) $\delta 7.44-7.37(\mathrm{~m}, 2 \mathrm{H}), 7.32-7.28$ (m, $2 \mathrm{H}), 7.20-7.13(\mathrm{~m}, 1 \mathrm{H}), 5.46-5.37(\mathrm{~m}, 1 \mathrm{H}), 2.11(\mathrm{q}, J=7.1 \mathrm{~Hz}, 2 \mathrm{H}), 1.85-1.74(\mathrm{~m}, 2 \mathrm{H})$, $1.49-1.36(\mathrm{~m}, 4 \mathrm{H}), 0.91(\mathrm{t}, J=7.2 \mathrm{~Hz}, 3 \mathrm{H}), 0.01(\mathrm{~s}, 9 \mathrm{H}) .{ }^{13} \mathrm{C}$ NMR $(101 \mathrm{MHz}$, Chloroform- $d)$ $\delta 204.1,138.5,128.0 \times 2,126.1,126.1 \times 2,102.5,93.3,31.6,29.2,22.4,19.0,14.0,-1.1 \times 3 . H R M S(E S I) ~ m / z: ~[M$ $+\mathrm{H}]^{+}$Calcd for $\mathrm{C}_{17} \mathrm{H}_{27} \mathrm{Si} 259.1877$; Found: 259.1882 .

\section{(2,5-Diphenylpenta-2,3-dien-1-yl)trimethylsilane (1q)}

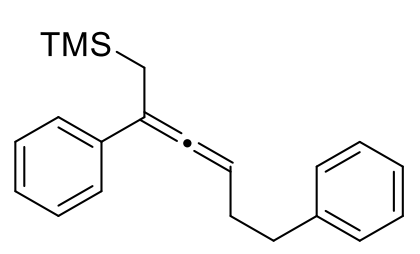

The title compound was prepared according to GP2 and isolated as a colorless oil (1.26 g, $4.11 \mathrm{mmol}, 41 \%) .{ }^{1} \mathrm{H}$ NMR (400 MHz, Chloroform- $\left.d\right) \delta 7.38-7.31(\mathrm{~m}, 6 \mathrm{H})$, $7.31-7.21(\mathrm{~m}, 4 \mathrm{H}), 5.51$ (tt, $J=6.7,2.4 \mathrm{~Hz}, 1 \mathrm{H}), 2.86(\mathrm{td}, J=7.5,2.1 \mathrm{~Hz}, 2 \mathrm{H})$, $2.56-2.44(\mathrm{~m}, 2 \mathrm{H}), 1.82(\mathrm{~d}, J=2.5 \mathrm{~Hz}, 2 \mathrm{H}), 0.07$ (s, 9H). ${ }^{13} \mathrm{C}$ NMR $(101 \mathrm{MHz}$,

Chloroform- $d$ ) $\delta 204.3,141.7,138.1,128.6 \times 2,128.3 \times 2,128.0 \times 2,126.2,126.1 \times 2,125.8,103.0,92.5,35.7$, 31.5, 18.9, -1.1 × 3. HRMS (ESI) m/z: $[\mathrm{M}+\mathrm{H}]^{+}$Calcd for $\mathrm{C}_{21} \mathrm{H}_{27} \mathrm{Si} 307.1877$; Found: 307.1882.

\subsection{Preparation of tertiary hydrocarbons $2 b-2 q$}


<smiles>COC(=O)C(C)OC</smiles>

$2 b$<smiles>CCOC(=O)C(CC1CC1)C(=O)OCC</smiles>

$2 \mathrm{~g}$<smiles>CCOCC(CC)C(=O)OCC</smiles>

2c<smiles>CCOC(=O)C(Cc1ccccc1)C(=O)OCC</smiles>

2d<smiles>CCOCC(C[14C])C(=O)OCC</smiles>

$2 e$<smiles>CCOC(=O)C(F)C(=O)OCC</smiles>

$2 f$<smiles>CCOCC(CC1CCCC1)C(=O)OCC</smiles>

$2 h$<smiles>CCOC(=O)CCCC(OCC)C(=O)OCC</smiles>

2i<smiles>CCOCC(CC[OH2+])C(=O)OCC</smiles>

2j<smiles>CCOC(C)C(C)COC</smiles>

2k

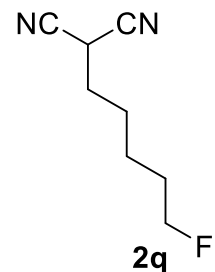

$2 q F$

2I

$2 \mathrm{~m}$

$2 n$<smiles>CC(C#N)C#N</smiles>

20<smiles>N#CC(C#N)CCC1OCCO1</smiles>

$2 p$

Compounds $\mathbf{2 b} \mathbf{- 2 d}, \mathbf{2 f}, \mathbf{2 k}$, and $\mathbf{2 l}$ were commercial available. Compounds $\mathbf{2 e}, \mathbf{2} \mathbf{g}-\mathbf{2} \mathbf{j}$ were prepared according to the general procedure $3 .^{[2]}$ Compound $\mathbf{2} \mathbf{m}^{[3]}, \mathbf{2} \mathbf{n}^{[4]}$, and $\mathbf{2} \mathbf{o}^{[5]}$ were prepared according to the known procedures. Compounds $\mathbf{2} \mathbf{p}$ and $\mathbf{2 q}$ were prepared according to the general procedure $4{ }^{[6]}$

\subsubsection{The general procedure 3 (GP3) for the synthesis of $2 \mathrm{e}, 2 \mathrm{~g}-2 \mathrm{j}^{[2]}$}

$$
\mathrm{EtO}_{2} \mathrm{C} \smile \mathrm{CO}_{2} \mathrm{Et}+\mathrm{R}-\mathrm{Br} \underset{\mathrm{THF}, \mathrm{rt}, 12 \mathrm{~h}}{\longrightarrow} \mathrm{EtO}_{2} \mathrm{C}_{\mathrm{R}} \mathrm{CO}_{2} \mathrm{Et}
$$

To a stirred solution of diethyl malonate $(1.60 \mathrm{~g}, 10 \mathrm{mmol}, 1.0$ equiv) in THF (10 mL) was added $\mathrm{NaH}(60 \%$ in mineral oil, $0.42 \mathrm{mg}, 10.5 \mathrm{mmol}, 1.05$ equiv) in several portions at room temperature. The mixture was stirred at this temperature for $10 \mathrm{~min}$. $\mathrm{R}-\mathrm{Br}(1.0$ equiv, $10 \mathrm{mmol}$ ) was slowly added to the mixture, then the solution was stirred for $12 \mathrm{~h}$ at room temperature. After completion of the reaction (as judged by TLC), the reaction mixture was quenched with saturated aqueous $\mathrm{NH}_{4} \mathrm{Cl}(10 \mathrm{~mL})$ and extracted with EtOAc $(30 \mathrm{~mL} \times 3)$. The combined organic phase was washed with brine $(10 \mathrm{~mL})$, dried over $\mathrm{Na}_{2} \mathrm{SO}_{4}$, filtered and concentrated in vacuo. The residue was purified by flash column chromatography (petroleum ether/EtOAc) to give $\alpha$-substituted malonates $\mathbf{2 e}, \mathbf{2 g}-\mathbf{2 j}$.

\section{Diethyl 2-(2-oxopropyl)malonate (2e)}

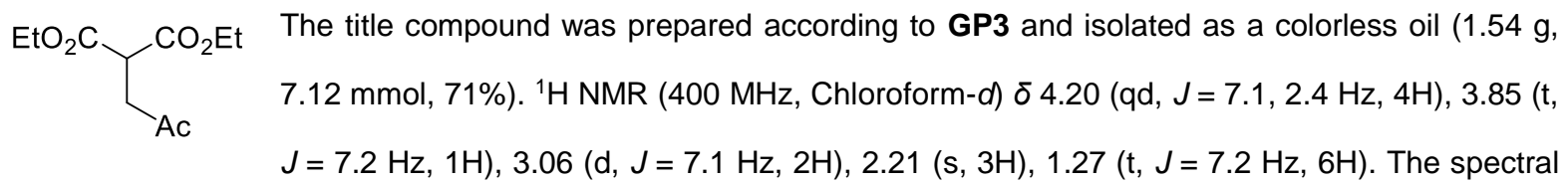
data matched those reported previously. ${ }^{[7]}$

\section{Diethyl 2-(cyclopropylmethyl)malonate (2g)}




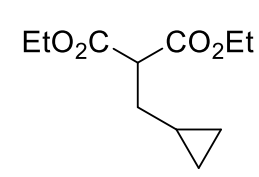

The title compound was prepared according to GP3 and isolated as a colorless oil (1.61 g, $7.51 \mathrm{mmol}, 75 \%) .{ }^{1} \mathrm{H}$ NMR (400 MHz, Chloroform- $d$ ) $\delta 4.20$ (q, $\left.J=7.1 \mathrm{~Hz}, 4 \mathrm{H}\right), 3.44$ (t, $J=$ $7.5 \mathrm{~Hz}, 1 \mathrm{H}), 1.80(\mathrm{t}, J=7.3 \mathrm{~Hz}, 2 \mathrm{H}), 1.27(\mathrm{t}, J=7.1 \mathrm{~Hz}, 6 \mathrm{H}), 0.79-0.68(\mathrm{~m}, 1 \mathrm{H}), 0.49-0.41$

$(\mathrm{m}, 2 \mathrm{H}), 0.12-0.06(\mathrm{~m}, 2 \mathrm{H})$. The spectral data matched those reported previously. ${ }^{[2]}$

\section{Diethyl 2-(cyclopentylmethyl)malonate $(2 \mathrm{~h})$}

$\mathrm{EtO}_{2} \mathrm{C} \mathrm{CO}_{2} \mathrm{Et}$ The title compound was prepared according to GP3 and isolated as a colorless oil $(1.52 \mathrm{~g}$, $6.27 \mathrm{mmol}, 63 \%) .{ }^{1} \mathrm{H}$ NMR (400 MHz, Chloroform- $d$ ) $\delta 4.19$ (q, $J=7.2 \mathrm{~Hz}, 4 \mathrm{H}$ ), 3.44 (t, $J=7.7$ $\mathrm{Hz}, 1 \mathrm{H}), 1.79(\mathrm{t}, J=7.3 \mathrm{~Hz}, 2 \mathrm{H}), 1.75-1.65(\mathrm{~m}, 4 \mathrm{H}), 1.26(\mathrm{t}, J=7.1 \mathrm{~Hz}, 6 \mathrm{H}), 1.22-1.10(\mathrm{~m}$, $3 \mathrm{H}), 0.91$ (dd, $J=12.9,9.7 \mathrm{~Hz}, 2 \mathrm{H}$ ). The spectral data matched those reported previously. ${ }^{[2]}$

\section{Triethyl butane-1,1,4-tricarboxylate (2i)}<smiles>CCOCCCC(COCC)C(=O)OCC</smiles>

The title compound was prepared according to GP3 and isolated as a colorless oil (1.46 g, $5.32 \mathrm{mmol}, 53 \%) .{ }^{1} \mathrm{H}$ NMR (400 MHz, Chloroform- $d$ ) $\delta 4.25-4.15(\mathrm{~m}, 4 \mathrm{H}), 4.11$ (q, $J=7.1$ $\mathrm{Hz}, 2 \mathrm{H}), 3.33(\mathrm{t}, J=7.5 \mathrm{~Hz}, 1 \mathrm{H}), 2.33(\mathrm{t}, J=7.5 \mathrm{~Hz}, 2 \mathrm{H}), 1.97-1.87(\mathrm{~m}, 2 \mathrm{H}), 1.69-1.60$ $(\mathrm{m}, 2 \mathrm{H}), 1.31-1.20(\mathrm{~m}, 9 \mathrm{H})$. The spectral data matched those reported previously. ${ }^{[8]}$

\section{Diethyl 2-(2-((tert-butyldimethylsilyl)oxy)ethyl)malonate (2j)}

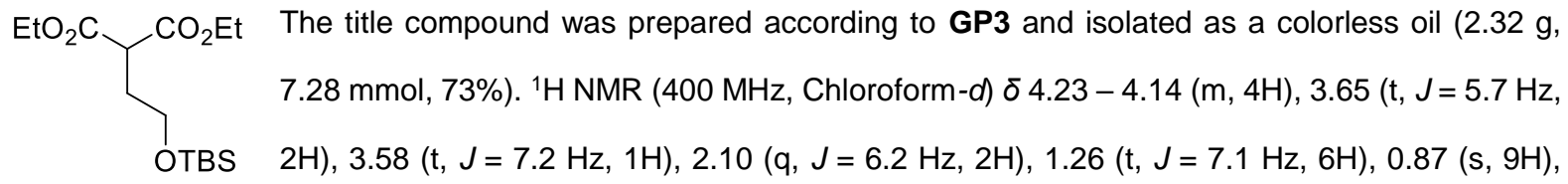

$0.02(\mathrm{~s}, 6 \mathrm{H})$. The spectral data matched those reported previously. ${ }^{[9]}$

\subsubsection{Synthesis of $2 \mathrm{~m}^{[3]}$}

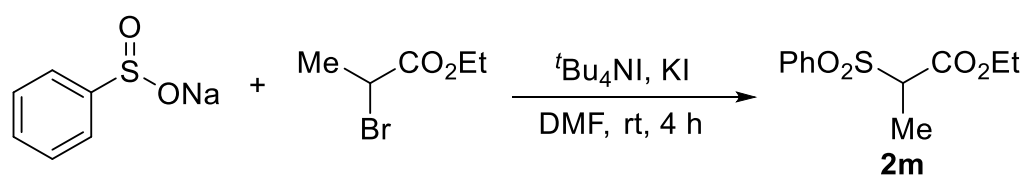

To a solution of sodium benzenesulfinate $(0.82 \mathrm{~g}, 5 \mathrm{mmol}, 1.0$ equiv) in DMF (30 mL) was added ethyl 2bromopropionate $(1.09 \mathrm{~g}, 6 \mathrm{mmol}, 1.2$ equiv), tetrabutylammonium iodide (184.7 $\mathrm{mg}, 0.5 \mathrm{mmol}, 0.1$ equiv), potassium iodide $(1.00 \mathrm{~g}, 6 \mathrm{mmol}, 1.2$ equiv). The reaction mixture was stirred at room temperature for $4 \mathrm{~h}$ and then quenched with saturated aqueous $\mathrm{NaHCO}_{3}(10 \mathrm{~mL})$. The mixture was extracted with $\mathrm{CH}_{2} \mathrm{Cl}_{2}(20 \mathrm{~mL} \times 3)$. The combined organic extracts were washed with brine $(10 \mathrm{~mL})$, dried over $\mathrm{Na}_{2} \mathrm{SO}_{4}$. Removal of the solvent in vacuo gave $2 \mathrm{~m}$ (831.1 mg, $3.43 \mathrm{mmol}, 69 \%) .{ }^{1} \mathrm{H}$ NMR (400 MHz, Chloroform- $d$ ) $\delta 7.89$ (dd, J = 8.4, $\left.1.3 \mathrm{~Hz}, 2 \mathrm{H}\right), 7.74-$ $7.66(\mathrm{~m}, 1 \mathrm{H}), 7.58(\mathrm{t}, J=7.7 \mathrm{~Hz}, 2 \mathrm{H}), 4.11(\mathrm{q}, J=7.1 \mathrm{~Hz}, 2 \mathrm{H}), 4.05(\mathrm{q}, J=7.2 \mathrm{~Hz}, 1 \mathrm{H}), 1.57(\mathrm{~d}, J=7.1 \mathrm{~Hz}, 3 \mathrm{H})$, $1.16(\mathrm{t}, J=7.2 \mathrm{~Hz}, 3 \mathrm{H})$. The spectral data matched those reported previously. ${ }^{[3]}$ 
3.2.3 Synthesis of $2 \mathbf{n}^{[4]}$

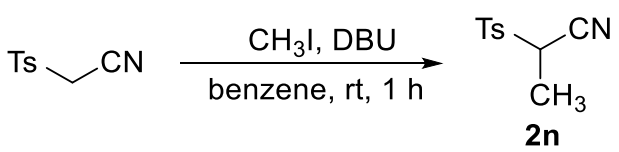

To a stirred solution of tosylacetonitrile ( $390.5 \mathrm{mg}, 2 \mathrm{mmol}, 1.0$ equiv) and DBU (334.9 mg, $2 \mathrm{mmol}, 1.1$ equiv) in benezene $(10 \mathrm{~mL})$ was added $\mathrm{CH}_{3} \mathrm{l}(567.8 \mathrm{mg}, 4 \mathrm{mmol}, 2.0$ equiv) and the solution was stirred at room temperature for $1 \mathrm{~h}$. The precipitated DBU-HI salt was removed by filtration and the residue was concentrated in vacuo to give the crude product. The crude product was purified by flash column chromatography (petroleum ether/EtOAc) to give a colorless liquid $2 \mathrm{n}(226.0 \mathrm{mg}, 1.08 \mathrm{mmol}, 54 \%) .{ }^{1} \mathrm{H}$ NMR $(400 \mathrm{MHz}$, Chloroform- $d$ ) $\delta 7.88$ (d, $J=8.1 \mathrm{~Hz}, 2 \mathrm{H}), 7.43(\mathrm{~d}, J=8.0 \mathrm{~Hz}, 2 \mathrm{H}), 3.97(\mathrm{q}, J=7.2 \mathrm{~Hz}, 1 \mathrm{H}), 2.48(\mathrm{~s}, 3 \mathrm{H}), 1.69$ (d, $J=7.2 \mathrm{~Hz}, 3 \mathrm{H})$.

\subsubsection{Synthesis of $20^{[5]}$}

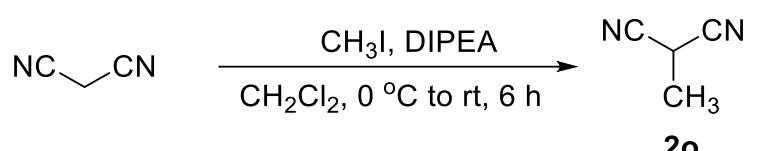

To a solution of malononitrile ( $330.3 \mathrm{mg}, 5 \mathrm{mmol}, 1.0$ equiv) and $N, N$-diisopropylethylamine (DIPEA, $646.2 \mathrm{mg}$, $5 \mathrm{mmol}, 1.0$ equiv) in $\mathrm{CH}_{2} \mathrm{Cl}_{2}(10 \mathrm{~mL})$ was added $\mathrm{CH}_{3} \mathrm{l}\left(709.7 \mathrm{mg}, 5 \mathrm{mmol}, 1.0\right.$ equiv) at $0{ }^{\circ} \mathrm{C}$, then the solution was allowed to warm to room temperature and stirred for $4 \mathrm{~h}$. The reaction mixture was concentrated in vacuo to give crude product, which was purified by flash column chromatography (petroleum ether/EtOAc) to afford a colorless liquid 20 (152.2 mg, $1.9 \mathrm{mmol}, 38 \%)^{1} \mathrm{H}$ NMR (400 MHz, Chloroform- $\left.d\right) \delta 3.79$ (q, $\left.J=7.3 \mathrm{~Hz}, 1 \mathrm{H}\right), 1.79$ (d, $J=7.3$ $\mathrm{Hz}, 3 \mathrm{H})$. The spectral data matched those reported previously. ${ }^{[5]}$

\subsubsection{The general procedure 4 (GP4) for the synthesis of $2 p$ and $2 q^{[6]}$}

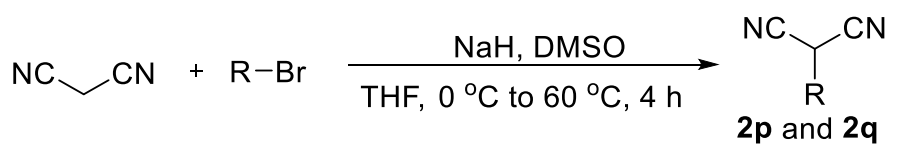

To a stirred suspension of $\mathrm{NaH}$ ( $60 \%$ in mineral oil, $0.40 \mathrm{~g}, 10 \mathrm{mmol}, 1.0$ equiv) in THF ( $30 \mathrm{~mL}$ ) at $0{ }^{\circ} \mathrm{C}$ was added a solution of malononitrile $(0.65 \mathrm{~g}, 10 \mathrm{mmol}, 1.0$ equiv) in THF (10 mL). After $30 \mathrm{~min}$, DMSO (5 mL) and a solution of alkyl bromide (10 mmol 1.0 equiv) in THF $(10 \mathrm{~mL})$ were added, and the mixture was heated to $60^{\circ} \mathrm{C}$ and stirred for $4 \mathrm{~h}$. The reaction mixture was quenched with saturated aqueous $\mathrm{NH}_{4} \mathrm{Cl}(10 \mathrm{~mL})$, and extracted with $\mathrm{Et}_{2} \mathrm{O}(30 \mathrm{~mL} \times 3)$. The organic layer was washed with $\mathrm{H}_{2} \mathrm{O}(10 \mathrm{~mL})$ and brine $(10 \mathrm{~mL})$, dried over $\mathrm{Na}_{2} \mathrm{SO}_{4}$, and concentrated in vacuo. The residue was purified by silica gel column chromatography (petroleum ether/EtOAc) to give $2 \mathbf{p}$ and $\mathbf{2 q}$.

\section{2-(2-(1,3-Dioxolan-2-yl)ethyl)malononitrile (2p)}


NC_CN The title compound was prepared according to GP4 and isolated as a colorless oil (701.3 mg, 4.22 mmol, 42\%). ${ }^{1} \mathrm{H}$ NMR (400 MHz, Chloroform- $\left.d\right) \delta 4.93(\mathrm{t}, J=3.8 \mathrm{~Hz}, 1 \mathrm{H}), 4.05(\mathrm{t}, J=7.5 \mathrm{~Hz}, 1 \mathrm{H})$, $4.02-3.93(\mathrm{~m}, 2 \mathrm{H}), 3.92-3.81(\mathrm{~m}, 2 \mathrm{H}), 2.19(\mathrm{q}, J=7.1 \mathrm{~Hz}, 2 \mathrm{H}), 1.99(\mathrm{td}, J=6.9,3.8 \mathrm{~Hz}, 2 \mathrm{H}) .{ }^{13} \mathrm{C}$ NMR (101 MHz, Chloroform-d) $\delta 112.8$ × 2, 102.7, 65.2 × 2, 29.4, 25.4, 22.4. HRMS (ESI) m/z: [M

$+\mathrm{H}]^{+}$Calcd for $\mathrm{C}_{8} \mathrm{H}_{11} \mathrm{~N}_{2} \mathrm{O}_{2}$ 167.0815; Found: 167.0820 .

\section{2-(5-Fluoropentyl)malononitrile (2q)}

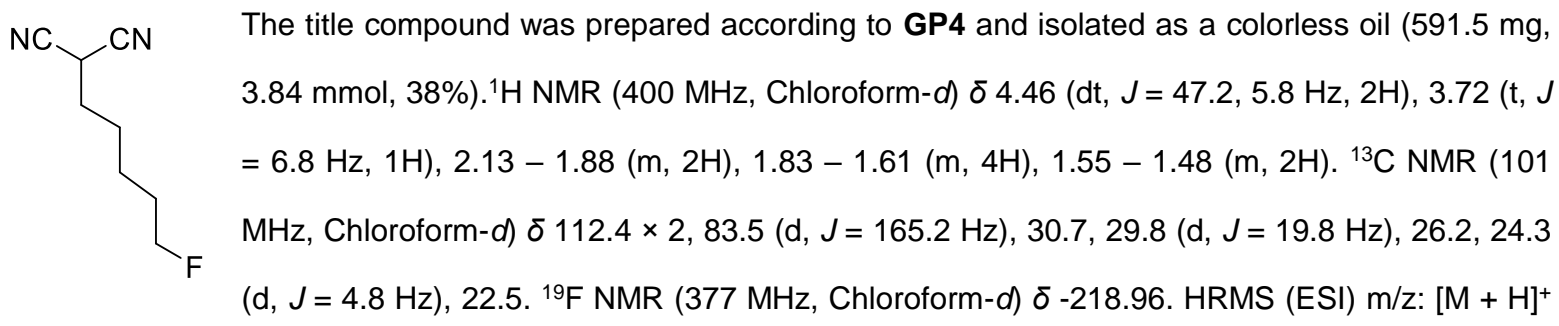

Calcd for $\mathrm{C}_{8} \mathrm{H}_{12} \mathrm{FN} 2$ 155.0979; Found: 155.0982.

\section{The general procedure 5 (GP5) for the synthesis of 3}

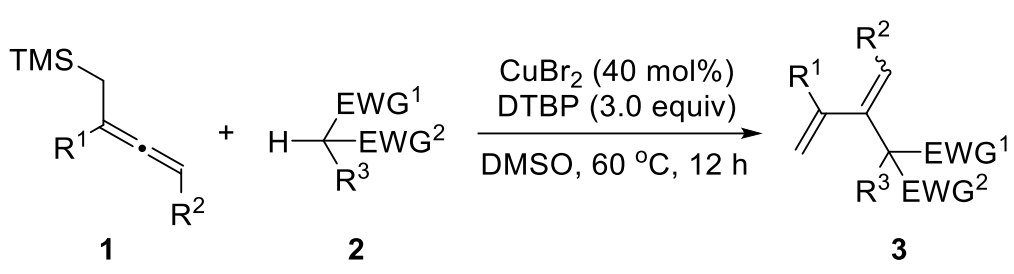

An oven-dried $10 \mathrm{~mL}$ Schlenk tube equipped with a stir bar was charged with $\mathrm{CuBr}_{2}(13.4 \mathrm{mg}, 0.06 \mathrm{mmol}, 40$ mol\%) and the tube was evacuated and backfilled with $\mathrm{N}_{2}$ for 3 times. 1 (0.21 mmol, 1.4 equiv), 2 (0.15 mmol, 1.0 equiv), DTBP (65.8 mg, $0.45 \mathrm{mmol}, 3.0$ equiv) and DMSO (1.5 mL) were successively added via syringe under $\mathrm{N}_{2}$ atmosphere. The sealed tube was placed into a preheated oil bath at $60^{\circ} \mathrm{C}$ with stirring for $12 \mathrm{~h}$. After cooling to room temperature, the mixture was diluted with water $(10 \mathrm{~mL})$. The layers were separated and the aqueous layer was extracted with $\mathrm{CH}_{2} \mathrm{Cl}_{2}(10 \mathrm{~mL} \times 3)$. The combined organic layers were rinsed with brine $(10 \mathrm{~mL})$, dried over $\mathrm{Na}_{2} \mathrm{SO}_{4}$, and concentrated in vacuo. The resultant residue was purified by flash column chromatography (petroleum ether/EtOAc) on silica gel to afford 3.

\section{Scale-up synthesis of product 3aa}

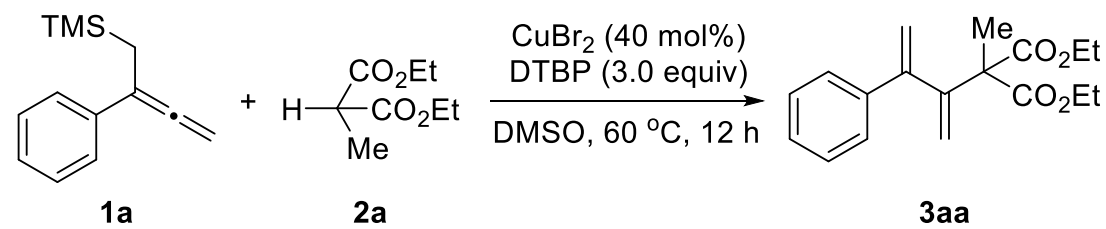

An oven-dried $50 \mathrm{~mL}$ Schlenk tube equipped with a stir bar was charged with $\mathrm{CuBr}_{2}(89.3 \mathrm{mg}, 0.4 \mathrm{mmol}, 40$ mol\%) and the tube was evacuated and backfilled with $\mathrm{N}_{2}$ for 3 times. 1a (283.3 mg, 1.4 mmol, 1.4 equiv), 2a 
(174.2 mg, $1 \mathrm{mmol}, 1.0$ equiv), DTBP (438.7 mg, $3 \mathrm{mmol}, 3.0$ equiv) and DMSO (10 mL) were successively added via syringe under $\mathrm{N}_{2}$ atmosphere. The sealed tube was placed into a preheated oil bath at $60{ }^{\circ} \mathrm{C}$ with stirring for $12 \mathrm{~h}$. After cooling to room temperature, the mixture was diluted with water (40 mL). The layers were separated and the aqueous layer was extracted with $\mathrm{CH}_{2} \mathrm{Cl}_{2}(30 \mathrm{~mL} \times 3)$. The combined organic layers were rinsed with brine $(10 \mathrm{~mL})$, dried over anhydrous $\mathrm{Na}_{2} \mathrm{SO}_{4}$, filtered and the filtrate was concentrated in vacuo. The resultant residue was purified by flash column chromatography (petroleum ether/EtOAc) on silica gel to afford the coupling product 3aa $(214.7 \mathrm{mg}, 0.71 \mathrm{mmol}, 71 \%)$.

\section{Derivatization reactions of product 3aa}

\subsection{Synthesis of compound 4}<smiles>C=C(C(=C)C(C)(COCC)C(=O)OCC)c1ccccc1</smiles>

3aa<smiles>O=C1C=CC(=O)N1c1ccccc1</smiles>

O toluene, $170^{\circ} \mathrm{C}, 24 \mathrm{~h}$<smiles>CCOC(=O)C(C)(C(=O)OCC)C1=C(c2ccccc2)CC2C(=O)N(c3ccccc3)C(=O)C2C1</smiles>

4, $81 \%$

To a solution of 3 aa $(30.2 \mathrm{mg}, 0.10 \mathrm{mmol}, 1.0$ equiv) in toluene $(3.0 \mathrm{~mL})$ was added $N$-phenylmaleimide (34.6 $\mathrm{mg}, 0.20 \mathrm{mmol}, 2.0$ equiv), then the mixture was stirred at $170{ }^{\circ} \mathrm{C}$ for $24 \mathrm{~h}$. Toluene was removed in vacuo, and the reaction mixture was purified by flash column chromatography (petroleum ether/EtOAc) on silica gel to afford the compound 4 as a colorless oil (38.5 mg, $0.081 \mathrm{mmol}, 81 \%)$. ${ }^{1} \mathrm{H}$ NMR (400 MHz, Chloroform- $d$ ) $\delta 7.51-7.43$ $(\mathrm{m}, 2 \mathrm{H}), 7.42-7.36(\mathrm{~m}, 1 \mathrm{H}), 7.35-7.29(\mathrm{~m}, 2 \mathrm{H}), 7.27(\mathrm{t}, J=1.6 \mathrm{~Hz}, 1 \mathrm{H}), 7.24-7.15(\mathrm{~m}, 2 \mathrm{H}), 7.15-7.04(\mathrm{~m}, 2 \mathrm{H})$, $3.89(\mathrm{q}, J=7.1 \mathrm{~Hz}, 2 \mathrm{H}), 3.68-3.47(\mathrm{~m}, 2 \mathrm{H}), 3.37-3.28(\mathrm{~m}, 2 \mathrm{H}), 2.90(\mathrm{dd}, J=15.0,3.0 \mathrm{~Hz}, 1 \mathrm{H}), 2.73-2.66(\mathrm{~m}$, 2H), $2.61-2.49(\mathrm{~m}, 1 \mathrm{H}), 1.43(\mathrm{~s}, 3 \mathrm{H}), 1.14(\mathrm{t}, J=7.1 \mathrm{~Hz}, 3 \mathrm{H}), 1.04(\mathrm{t}, J=7.2 \mathrm{~Hz}, 3 \mathrm{H}) .{ }^{13} \mathrm{C} \mathrm{NMR}(101 \mathrm{MHz}$, Chloroform- $d$ ) $\delta 178.6,178.2,170.7,170.5,141.2,137.6,132.1,131.4,129.2 \times 2,128.7,128.3 \times 2,127.9 \times 2$, 127.2, 126.6 × 2, 62.61, 61.4, 59.9, 40.2, 40.1, 34.1, 29.3, 22.4, 13.8, 13.6. HRMS (ESI) m/z: [M + H]+ Calcd for $\mathrm{C}_{28} \mathrm{H}_{30} \mathrm{NO}_{6}$ 476.2068; Found: 476.2073.

\subsection{Synthesis of compound 5}<smiles>C=C(C(=C)C(C)(C)OCC)c1ccccc1</smiles>

$3 a a$<smiles>O=C1C=CC(=O)O1</smiles>

toluene, $170^{\circ} \mathrm{C}, 36 \mathrm{~h}$<smiles>CCOC(=O)C(C)(C)C1=C(c2ccccc2)CC2C(=O)OC(=O)C2C1</smiles>

5, $72 \%$

To a solution of $3 \mathbf{a a}(30.2 \mathrm{mg}, 0.10 \mathrm{mmol}, 1.0$ equiv) in toluene $(3 \mathrm{~mL})$, maleic anhydride $(19.6 \mathrm{mg}, 0.20 \mathrm{mmol}$, 2.0 equiv) was added, then the mixture stirred at $170^{\circ} \mathrm{C}$ for $36 \mathrm{~h}$. Toluene was removed in vacuo, and the reaction mixture was purified by flash column chromatography (petroleum ether/EtOAc) on silica gel to afford the compound 
5 as a colorless oil $(29.0 \mathrm{mg}, 0.072 \mathrm{mmol}, 72 \%) .{ }^{1} \mathrm{H}$ NMR $(400 \mathrm{MHz}$, Chloroform- $d$ ) $\delta 7.27(\mathrm{~d}, J=6.7 \mathrm{~Hz}, 2 \mathrm{H}), 7.24$ - $7.18(\mathrm{~m}, 1 \mathrm{H}), 7.09-6.99(\mathrm{~m}, 2 \mathrm{H}), 3.94-3.86(\mathrm{~m}, 1 \mathrm{H}), 3.84-3.75(\mathrm{~m}, 3 \mathrm{H}), 3.50-3.40(\mathrm{~m}, 2 \mathrm{H}), 2.79(\mathrm{dd}, J=$ 15.0, $2.4 \mathrm{~Hz}, 1 \mathrm{H}), 2.70-2.60(\mathrm{~m}, 2 \mathrm{H}), 2.60-2.51(\mathrm{~m}, 1 \mathrm{H}), 1.38(\mathrm{~s}, 3 \mathrm{H}), 1.16(\mathrm{dt}, J=13.9,7.1 \mathrm{~Hz}, 6 \mathrm{H}) .{ }^{13} \mathrm{C} \mathrm{NMR}$ (101 MHz, Chloroform- $d$ ) $\delta 173.7,173.4,170.6,170.4,140.9,137.8,131.7,128.1 \times 2,128.0 \times 2,127.5,61.7,61.7$, 60.0, 40.8, 40.4, 33.4, 29.4, 22.1, 13.7, 13.7. HRMS (ESI) m/z: $[\mathrm{M}+\mathrm{H}]^{+}$Calcd for $\mathrm{C}_{22} \mathrm{H}_{25} \mathrm{O}_{7}$ 401.1595; Found: 401.1600 .

\subsection{Synthesis of compound 6}<smiles>C=C(C(=C)C(C)(C(C)=O)C(=O)OCC)c1ccccc1</smiles>

3aa<smiles>N#CC(C#N)=C(C#N)C#N</smiles>

toluene, $160^{\circ} \mathrm{C}, 12 \mathrm{~h}$<smiles>CCOC(=O)C(C)(C)C1=C(c2ccccc2)C[C@@](C)(C#N)[C@H](C#N)C1</smiles>

6, $71 \%$

To a solution of 3 aa $(30.2 \mathrm{mg}, 0.10 \mathrm{mmol}, 1.0$ equiv) in toluene $(3 \mathrm{~mL})$ was added tetracyanoethylene $(25.6$ $\mathrm{mg}, 0.20 \mathrm{mmol}, 2.0$ equiv), then the mixture stirred at $160{ }^{\circ} \mathrm{C}$ for $12 \mathrm{~h}$. Toluene was removed in vacuo, and the reaction mixture was purified by flash column chromatography (petroleum ether/EtOAc) on silica gel to afford the compound 6 as a colorless oil (30.5 mg, $0.071 \mathrm{mmol}, 71 \%) .{ }^{1} \mathrm{H}$ NMR (400 MHz, Chloroform- $d$ ) $\delta 7.40-7.32$ (m, 3H), 7.09 (dd, J = 6.6, $2.9 \mathrm{~Hz}, 2 \mathrm{H}), 4.21-3.89(\mathrm{~m}, 4 \mathrm{H}), 3.31(\mathrm{~s}, 2 \mathrm{H}), 3.27(\mathrm{~s}, 2 \mathrm{H}), 1.28-1.22(\mathrm{~m}, 9 \mathrm{H}) .{ }^{13} \mathrm{C} \mathrm{NMR}$ $(101 \mathrm{MHz}$, Chloroform- $d) \delta 169.6 \times 2,137.0,131.9,129.1,128.9 \times 2,128.1 \times 2,126.2,110.4 \times 2,110.2 \times 2,62.4$ $\times 2,59.5,40.5,38.0,37.7,35.1,22.1,13.8 \times 2$. HRMS $(E S I) ~ m / z:[M+H]^{+}$Calcd for $\mathrm{C}_{24} \mathrm{H}_{23} \mathrm{~N}_{4} \mathrm{O}_{4} 431.1714$; Found: 431.1719

\subsection{Synthesis of compound 7}

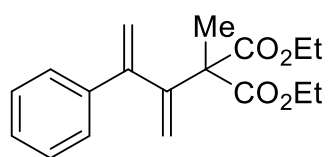

$3 a a$

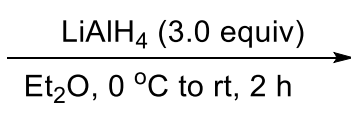

Lithium aluminium hydride ( $11.4 \mathrm{mg}, 0.30 \mathrm{mmol}, 3.0$ equiv) was taken up in $\mathrm{Et}_{2} \mathrm{O}(1 \mathrm{~mL})$ and then cooled to 0 ${ }^{\circ} \mathrm{C}$. To the $0{ }^{\circ} \mathrm{C}$ stirred grey mixture was added dropwise 3 aa $\left(30.2 \mathrm{mg}, 0.1 \mathrm{mmol}, 1.0\right.$ equiv) in a solution of $\mathrm{Et}_{2} \mathrm{O}$ $(1 \mathrm{~mL})$. The resulting mixture was warmed to room temperature and stirred for $2 \mathrm{~h}$. The mixture was cooled back to $0{ }^{\circ} \mathrm{C}$ and carefully quenched with $\mathrm{H}_{2} \mathrm{O}(5 \mathrm{~mL})$ to give a white milky mixture, which was then extracted with $\mathrm{Et}_{2} \mathrm{O}$ $(10 \mathrm{~mL} \times 3)$. The combined organic layers were washed with brine $(5 \mathrm{~mL})$ and purified by flash column chromatography $\left(\mathrm{CH}_{2} \mathrm{Cl}_{2} / \mathrm{CH}_{3} \mathrm{OH}\right)$ on silica gel to afford the compound 7 as a colorless oil (19.9 mg, $0.091 \mathrm{mmol}$, 91\%). ${ }^{1} \mathrm{H}$ NMR (400 MHz, Chloroform- $d$ ) $\delta 7.43-7.38(\mathrm{~m}, 2 \mathrm{H}), 7.34-7.27(\mathrm{~m}, 3 \mathrm{H}), 5.38(\mathrm{~d}, \mathrm{~J}=1.5 \mathrm{~Hz}, 1 \mathrm{H}), 5.36$ $(\mathrm{d}, J=1.8 \mathrm{~Hz}, 1 \mathrm{H}), 5.27(\mathrm{~d}, J=1.6 \mathrm{~Hz}, 1 \mathrm{H}), 5.09(\mathrm{~d}, J=1.8 \mathrm{~Hz}, 1 \mathrm{H}), 3.59(\mathrm{~d}, J=11.2 \mathrm{~Hz}, 2 \mathrm{H}), 3.48(\mathrm{~d}, J=11.0$ 
$\mathrm{Hz}, 2 \mathrm{H}$ ), 2.04 (br s, 2H), 0.93 (s, 3H). ${ }^{13} \mathrm{C}$ NMR (101 MHz, Chloroform-d) $\delta$ 151.5, 150.7, 140.4, $128.5 \times 2,128.0$, $126.3 \times 2,117.3,115.8,68.3 \times 2$, 45.8, 19.9. HRMS (ESI) m/z: $[\mathrm{M}+\mathrm{H}]^{+}$Calcd for $\mathrm{C}_{14} \mathrm{H}_{19} \mathrm{O}_{2} 219.1380$; Found: 219.1385 .

\subsection{Synthesis of compound 8}<smiles>C=C(C(=C)C(C)(C)OCC)c1ccccc1</smiles>

3aa

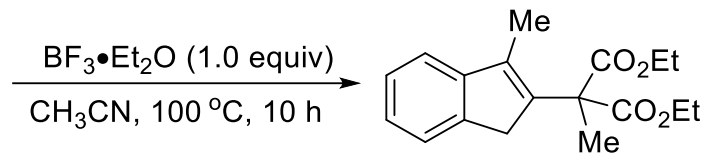

8, $74 \%$

To a solution of 3 aa $(30.2 \mathrm{mg}, 0.10 \mathrm{mmol}, 1.0$ equiv) in acetonitrile $(2 \mathrm{~mL})$ was added boron trifluoride etherate (14.6 mg, $0.10 \mathrm{mmol}, 1.0$ equiv, $46.5 \%$ in $\mathrm{Et}_{2} \mathrm{O}$ ). Then, the mixture stirred at $100{ }^{\circ} \mathrm{C}$ for $10 \mathrm{~h}$ under $\mathrm{N}_{2}$ atmosphere. Acetonitrile was removed in vacuo and the reaction mixture was purified by flash column chromatography (petroleum ether/EtOAc) on silica gel to afford the compound 8 as a colorless oil (22.4 mg, $0.074 \mathrm{mmol}, 74 \%)$. ${ }^{1} \mathrm{H}$ NMR (400 MHz, Chloroform- $d)$ d 7.39 (d, J = 7.3 Hz, 1H), $7.33-7.28(\mathrm{~m}, 2 \mathrm{H}), 7.20-7.16(\mathrm{~m}, 1 \mathrm{H}), 4.30-4.12(\mathrm{~m}$, 4H), $3.43(\mathrm{~d}, J=2.3 \mathrm{~Hz}, 2 \mathrm{H}), 2.05$ (t, $J=2.2 \mathrm{~Hz}, 3 \mathrm{H}), 1.77(\mathrm{~s}, 3 \mathrm{H}), 1.25(\mathrm{t}, J=7.1 \mathrm{~Hz}, 6 \mathrm{H}) .{ }^{13} \mathrm{C} \mathrm{NMR}(101 \mathrm{MHz}$, Chloroform- $d$ ) $\delta 171.5 \times 2,146.9,141.7,137.0,136.9,126.2,124.8,123.1,118.8,61.7 \times 2,56.0,39.7,22.5,14.0$ $\times 2$, 12.0. HRMS (ESI) m/z: [M + H] $]^{+}$Calcd for $\mathrm{C}_{18} \mathrm{H}_{23} \mathrm{O}_{4}$ 303.1591; Found: 303.1596.

\section{Mechanistic exploration experiments}

\subsection{Radical trapping experiments}<smiles>C=C=C(CC(C)(C)C)c1ccccc1</smiles>

(1.4 equiv)

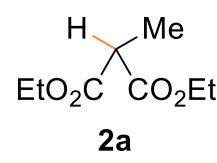

$(0.15 \mathrm{mmol})$

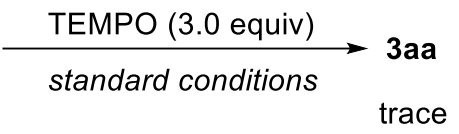
trace ace<smiles>C=[GeH2]</smiles>

An oven-dried $10 \mathrm{~mL}$ Schlenk tube equipped with a stir bar was charged with $\mathrm{CuBr}_{2}(13.4 \mathrm{mg}, 0.06 \mathrm{mmol}, 40$ mol\%), 2,2,6,6-tetramethylpiperidine $\mathrm{N}$-oxide (TEMPO, $70.3 \mathrm{mg}, 0.45 \mathrm{mmol}, 3.0$ equiv) and the tube was evacuated and backfilled with $\mathrm{N}_{2}$ for 3 times. 1a (42.5 mg, $0.21 \mathrm{mmol}, 1.4$ equiv), 2a (26.1 mg, $0.15 \mathrm{mmol}, 1.0$ equiv), DTBP (65.8 mg, $0.45 \mathrm{mmol}, 3.0$ equiv) and DMSO (1.5 mL) were successively added via syringe under $\mathrm{N}_{2}$ atmosphere. The mixture was stirred at $60^{\circ} \mathrm{C}$ for $12 \mathrm{~h}$ under $\mathrm{N}_{2}$ atmosphere. After cooling to room temperature, the mixture was diluted with water $(10 \mathrm{~mL})$. The layer was separated and the aqueous layer was extracted with $\mathrm{CH}_{2} \mathrm{Cl}_{2}(10 \mathrm{~mL} \times$ 3). The combined organic layers were rinsed with brine $(10 \mathrm{~mL})$, dried over $\mathrm{Na}_{2} \mathrm{SO}_{4}$, and concentrated in vacuo. Traces of the coupling product 3aa was observed by TLC analysis. The resultant residue was purified by flash column chromatography (petroleum ether/EtOAc) on silica gel to afford 9 (42.5 mg, $0.129 \mathrm{mmol}, 86 \%) .{ }^{1} \mathrm{H}$ NMR 
(400 MHz, Chloroform- $d) \delta 4.63-4.01(\mathrm{~m}, 4 \mathrm{H}), 1.73(\mathrm{~s}, 3 \mathrm{H}), 1.65-1.38(\mathrm{~m}, 6 \mathrm{H}), 1.27$ (t, J= 7.2 Hz, 6H), $1.22(\mathrm{~s}$, 6H), $1.02(\mathrm{~s}, 6 \mathrm{H}) \cdot{ }^{13} \mathrm{C}$ NMR (101 MHz, Chloroform- $\left.d\right) \delta 170.2 \times 2,85.3,61.5 \times 2,60.1 \times 2,40.6 \times 2,32.9 \times 2,20.5$ $\times 2,17.8,16.8,13.9 \times 2 . \mathrm{HRMS}(\mathrm{ESI}) \mathrm{m} / \mathrm{z}:[\mathrm{M}+\mathrm{H}]^{+}$Calcd for $\mathrm{C}_{17} \mathrm{H}_{32} \mathrm{NO}_{5}$ 330.2275; Found: 330.2281 .<smiles>C=C=C(C[As])c1ccccc1</smiles>

(1.4 equiv)<smiles>CCOC(C)C(OCC)OCC</smiles>

$2 a$

$(0.15 \mathrm{mmol})$

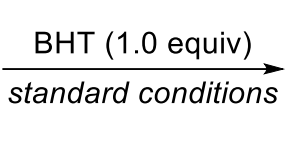

4<smiles>C=C(C(=C)C(C)(C)C(=O)OCC)c1ccccc1</smiles>

trace

An oven-dried $10 \mathrm{~mL}$ Schlenk tube equipped with a stir bar was charged with $\mathrm{CuBr}_{2}(13.4 \mathrm{mg}, 0.06 \mathrm{mmol}, 40$ mol\%), 2,6-di-tert-butyl-4-methylphenol (BHT, $33.1 \mathrm{mg}, 0.15 \mathrm{mmol}, 1.0$ equiv) and the tube was evacuated and backfilled with $\mathrm{N}_{2}$ for 3 times. 1a (42.5 mg, $0.21 \mathrm{mmol}, 1.4$ equiv), 2a (26.1 mg, $0.15 \mathrm{mmol}, 1.0$ equiv), DTBP (65.8 $\mathrm{mg}, 0.45 \mathrm{mmol}, 3.0$ equiv) and DMSO $(1.5 \mathrm{~mL})$ were successively added via syringe under $\mathrm{N}_{2}$ atmosphere. The mixture was stirred at $60^{\circ} \mathrm{C}$ for $12 \mathrm{~h}$ under $\mathrm{N}_{2}$ atmosphere. After cooling to room temperature, the mixture was diluted with water $(10 \mathrm{~mL})$. The layer was separated and the aqueous layer was extracted with $\mathrm{CH}_{2} \mathrm{Cl}_{2}(10 \mathrm{~mL} \times$ 3). The combined organic layers were rinsed with brine $(10 \mathrm{~mL})$, dried over $\mathrm{Na}_{2} \mathrm{SO}_{4}$, and concentrated in vacuo. Traces of the desired product 3aa was observed from ${ }^{1} \mathrm{H}$ NMR spectrometry of the crude mixture.

\subsection{By-product tracking experiment}<smiles>C=C=C(C[As])c1ccccc1</smiles>

(1.4 equiv)<smiles>CCOC(=O)C(C)OCC</smiles>

$2 a$

$(0.15 \mathrm{mmol})$

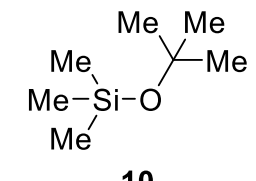

Detected by GC-MS

Following the standard conditions for the preparation of $\mathbf{3 a a}$, by-product $\mathbf{1 0}$ in the reaction crude mixture was detected by GC-MS analysis $\left(\mathrm{CH}_{2} \mathrm{Cl}_{2}\right.$ as solvent) in comparison with commercial available tertbutoxytrimethylsilane.
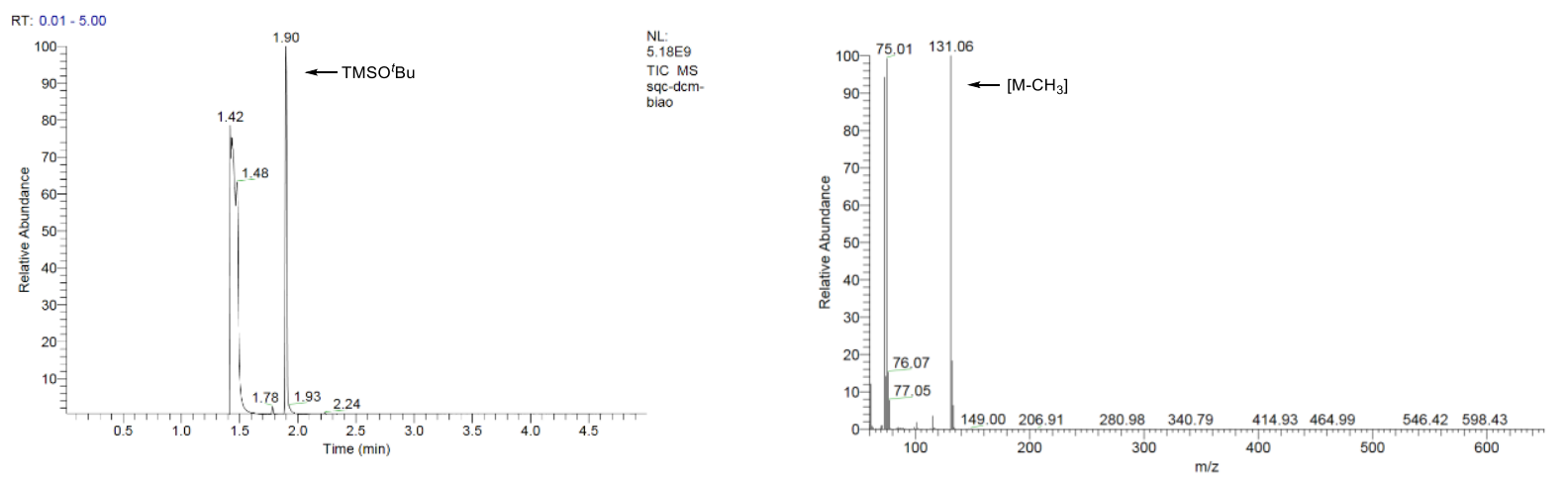

Figure S1. GC-MS spectrum for commercial available tert-butoxytrimethylsilane 

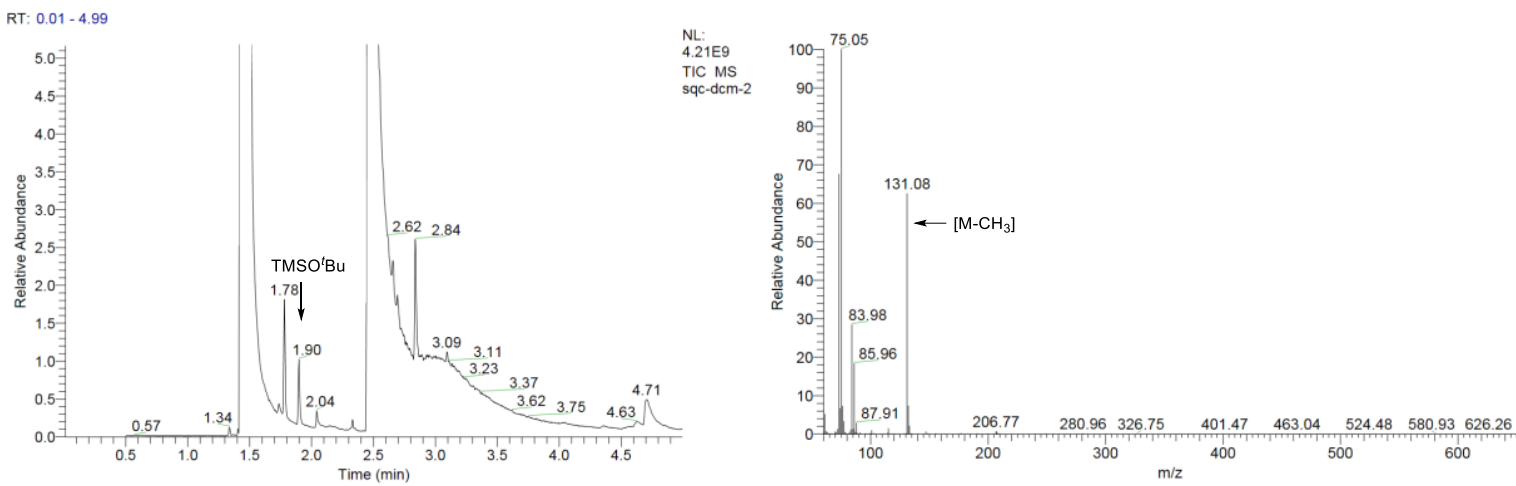

Figure S2. GC-MS spectrum of the reaction mixture

\section{NMR data of the products}

Diethyl 2-methyl-2-(3-phenylbuta-1,3-dien-2-yl)malonate (3aa)<smiles>C=C(C(=C)C(C)(OCC)C(C)=O)c1ccccc1</smiles>

The title compound was prepared according to GP5 and isolated as a yellow oil ( 31.8 mg, $0.105 \mathrm{mmol}, 70 \%) .{ }^{1} \mathrm{H}$ NMR (400 MHz, Chloroform- $\left.d\right) \delta 7.41-7.35(\mathrm{~m}, 2 \mathrm{H}), 7.32$ $-7.25(\mathrm{~m}, 3 \mathrm{H}), 5.44(\mathrm{~s}, 1 \mathrm{H}), 5.37(\mathrm{~d}, J=1.3 \mathrm{~Hz}, 1 \mathrm{H}), 5.32(\mathrm{~s}, 1 \mathrm{H}), 5.22(\mathrm{~d}, J=1.4 \mathrm{~Hz}$, 1H), 4.09 (dddd, $J=17.9,10.7,7.1,3.6 \mathrm{~Hz}, 4 \mathrm{H}), 1.52(\mathrm{~s}, 3 \mathrm{H}), 1.21$ (t, $J=7.1 \mathrm{~Hz}, 6 \mathrm{H}) .{ }^{13} \mathrm{C}$ NMR $(101 \mathrm{MHz}$, Chloroform-d) $\delta 171.1 \times 2,148.8,146.4,140.6,128.0 \times 2,127.6,127.2 \times 2,120.5,116.0,61.5 \times 2,59.4,22.0$, $13.9 \times 2$. HRMS (ESI) m/z: [M + H] $]^{+}$Calcd for $\mathrm{C}_{18} \mathrm{H}_{23} \mathrm{O}_{4}$ 303.1591; Found: 303.1588 .

\section{Diethyl 2-methyl-2-(3-(p-tolyl)buta-1,3-dien-2-yl)malonate (3ba)}<smiles>C=C(C(=C)C(C)(OCC)C(C)=O)c1ccc(C)cc1</smiles>

The title compound was prepared according to GP5 and isolated as a yellow oil (33.5 mg, $0.106 \mathrm{mmol}, 71 \%) .{ }^{1} \mathrm{H}$ NMR (400 MHz, Chloroform- $d$ ) $\delta 7.29$ (d, $J=8.2$ $\mathrm{Hz}, 2 \mathrm{H}), 7.10(\mathrm{~d}, J=7.9 \mathrm{~Hz}, 2 \mathrm{H}), 5.44(\mathrm{~d}, J=0.6 \mathrm{~Hz}, 1 \mathrm{H}), 5.36(\mathrm{~d}, J=1.5 \mathrm{~Hz}, 1 \mathrm{H})$, $5.33(\mathrm{~s}, 1 \mathrm{H}), 5.18(\mathrm{~d}, J=1.5 \mathrm{~Hz}, 1 \mathrm{H}), 4.20-4.03(\mathrm{~m}, 4 \mathrm{H}), 2.33(\mathrm{~s}, 3 \mathrm{H}), 1.53(\mathrm{~s}, 3 \mathrm{H}), 1.23(\mathrm{t}, J=7.1 \mathrm{~Hz}, 6 \mathrm{H}) .{ }^{13} \mathrm{C}$ NMR (101 MHz, Chloroform-d) $\delta 171.2 \times 2,148.6,146.4,137.7,137.4,128.7 \times 2,127.1 \times 2,120.3,115.2,61.5 \times$ 2, 59.3, 21.9, 21.1, $13.9 \times 2$. HRMS (ESI) m/z: [M + H] Calcd for $\mathrm{C}_{19} \mathrm{H}_{25} \mathrm{O}_{4} 317.1747$; Found: 317.1753.

\section{Diethyl 2-(3-(4-(tert-butyl)phenyl)buta-1,3-dien-2-yl)-2-methylmalonate (3ca)}<smiles>C=C(C(=C)C(C)(C)C(=O)OCC)c1ccc(C(C)(C)C)cc1</smiles>
The title compound was prepared according to GP5 and isolated as a yellow oil (35.1 mg, $0.098 \mathrm{mmol}, 65 \%) .{ }^{1} \mathrm{H}$ NMR (400 MHz, Chloroform- $\left.d\right) \delta 7.35-7.29$ (m, $4 \mathrm{H}), 5.44(\mathrm{~d}, J=0.7 \mathrm{~Hz}, 1 \mathrm{H}), 5.38(\mathrm{~d}, J=1.5 \mathrm{~Hz}, 1 \mathrm{H}), 5.33(\mathrm{~d}, J=0.8 \mathrm{~Hz}, 1 \mathrm{H})$, $5.18\left(\mathrm{~d}, J=1.5 \mathrm{~Hz}, 1 \mathrm{H}\right.$ ), 4.09 (qq, $J=10.7,7.1 \mathrm{~Hz}, 4 \mathrm{H}$ ), $1.55(\mathrm{~s}, 3 \mathrm{H}), 1.31(\mathrm{~s}, 9 \mathrm{H}), 1.21$ (t, $J=7.1 \mathrm{~Hz}, 6 \mathrm{H}$ ). ${ }^{13} \mathrm{C}$ NMR (101 MHz, Chloroform- $d) \delta 171.2 \times 2,150.6,148.5,146.4,137.6,126.8 \times 2,124.9 \times 2,120.3,115.3,61.5 \times$ 2, 59.4, 34.5, $31.3 \times$ 3, 22.0, $13.9 \times$ 2. HRMS (ESI) m/z: $[\mathrm{M}+\mathrm{H}]^{+}$Calcd for $\mathrm{C}_{22} \mathrm{H}_{31} \mathrm{O}_{4}$ 359.2217; Found: 359.2222. 
Diethyl 2-(3-(3-methoxyphenyl)buta-1,3-dien-2-yl)-2-methylmalonate (3da)<smiles>C=C(C(=C)C(C)(C)C(=O)OCC)c1cccc(OC)c1</smiles>

The title compound was prepared according to GP5 and isolated as a yellow oil (31.3 mg, $0.094 \mathrm{mmol}, 63 \%) .{ }^{1} \mathrm{H}$ NMR (400 MHz, Chloroform- $d$ ) $\delta 7.21$ (t, $J=7.9$ $\mathrm{Hz}, 1 \mathrm{H}), 6.99$ (dt, $J=7.7,1.3 \mathrm{~Hz}, 1 \mathrm{H}), 6.95$ (dd, $J=2.6,1.7 \mathrm{~Hz}, 1 \mathrm{H}), 6.81$ (ddd, $J$ $=8.1,2.6,0.9 \mathrm{~Hz}, 1 \mathrm{H}), 5.45(\mathrm{~s}, 1 \mathrm{H}), 5.40(\mathrm{~d}, J=1.4 \mathrm{~Hz}, 1 \mathrm{H}), 5.35(\mathrm{~s}, 1 \mathrm{H}), 5.23(\mathrm{~d}, J=1.5 \mathrm{~Hz}, 1 \mathrm{H}), 4.19-4.03(\mathrm{~m}$, 4H), $3.80(\mathrm{~s}, 3 \mathrm{H}), 1.53(\mathrm{~s}, 3 \mathrm{H}), 1.23(\mathrm{t}, J=7.1 \mathrm{~Hz}, 6 \mathrm{H}) \cdot{ }^{13} \mathrm{C}$ NMR $(101 \mathrm{MHz}$, Chloroform- $d) \delta 171.1 \times 2,159.3$, 148.6, 146.2, 142.0, 129.0, 120.6, 119.7, 116.1, 113.1, 112.9, $61.5 \times 2$, 59.3, 55.2, 21.9, $13.8 \times 2 . \mathrm{HRMS}(\mathrm{ESI})$ $\mathrm{m} / \mathrm{z}:[\mathrm{M}+\mathrm{H}]^{+}$Calcd for $\mathrm{C}_{19} \mathrm{H}_{25} \mathrm{O}_{5} 333.1697$; Found: 333.1702 .

\section{Diethyl 2-(3-(4-chlorophenyl)buta-1,3-dien-2-yl)-2-methylmalonate (3ea)}<smiles>C=C(C(=C)C(C)(C)C(=O)OCC)c1ccc(Cl)cc1</smiles>

The title compound was prepared according to GP5 and isolated as a yellow oil (32.3 $\mathrm{mg}, 0.096 \mathrm{mmol}, 64 \%) .{ }^{1} \mathrm{H}$ NMR (400 MHz, Chloroform-d) $\delta 7.35-7.31(\mathrm{~m}, 2 \mathrm{H})$, $7.27-7.25(\mathrm{~m}, 2 \mathrm{H}), 5.46(\mathrm{~s}, 1 \mathrm{H}), 5.37(\mathrm{~d}, J=1.3 \mathrm{~Hz}, 1 \mathrm{H}), 5.32(\mathrm{~s}, 1 \mathrm{H}), 5.24(\mathrm{~d}, J=$ $1.2 \mathrm{~Hz}, 1 \mathrm{H}), 4.20-3.99(\mathrm{~m}, 4 \mathrm{H}), 1.53(\mathrm{~s}, 3 \mathrm{H}), 1.22(\mathrm{t}, J=7.1 \mathrm{~Hz}, 6 \mathrm{H}) .{ }^{13} \mathrm{C}$ NMR $(101 \mathrm{MHz}$, Chloroform-d) $\delta 171.0$ $\times 2,147.7,146.0,139.1,133.5,128.6 \times 2,128.2 \times 2,120.8,116.5,61.6 \times 2,59.3,22.0,13.9 \times 2 . H R M S(E S I) ~ m / z:$ $[\mathrm{M}+\mathrm{H}]^{+}$Calcd for $\mathrm{C}_{18} \mathrm{H}_{22} \mathrm{ClO}_{4} 337.1201$; Found: 337.1207 .

\section{Diethyl 2-(3-(4-cyanophenyl)buta-1,3-dien-2-yl)-2-methylmalonate (3fa)}<smiles>C=C(C(=C)C(C)(C(C)=O)C(=O)OCC)c1ccc(C#N)cc1</smiles>

The title compound was prepared according to GP5 and isolated as a yellow oil (27.8 mg, $0.085 \mathrm{mmol}, 57 \%) .{ }^{1} \mathrm{H}$ NMR $(400 \mathrm{MHz}$, Chloroform- $d) \delta 7.63-7.56(\mathrm{~m}$, 2H), $7.54-7.48(\mathrm{~m}, 2 \mathrm{H}), 5.52(\mathrm{~s}, 1 \mathrm{H}), 5.49(\mathrm{~d}, J=0.9 \mathrm{~Hz}, 1 \mathrm{H}), 5.39(\mathrm{~d}, J=1.0 \mathrm{~Hz}$, $1 \mathrm{H}), 5.32(\mathrm{~s}, 1 \mathrm{H}), 4.11$ (qq, $J=10.7,7.1 \mathrm{~Hz}, 4 \mathrm{H}), 1.54(\mathrm{~s}, 3 \mathrm{H}), 1.22(\mathrm{t}, J=7.1 \mathrm{~Hz}, 6 \mathrm{H}) .{ }^{13} \mathrm{C} \mathrm{NMR}(101 \mathrm{MHz}$, Chloroform- $d$ ) $\delta 170.8 \times 2,147.4,145.5,145.3,132.0 \times 2,127.8 \times 2,121.5,118.8,118.7,111.2,61.7 \times 2,59.3$, 22.2, $13.9 \times 2$. HRMS (ESI) m/z: $[\mathrm{M}+\mathrm{H}]^{+}$Calcd for $\mathrm{C}_{19} \mathrm{H}_{22} \mathrm{NO}_{4}$ 328.1543; Found: 328.1549.

\section{Diethyl 2-methyl-2-(3-(4-(trifluoromethyl)phenyl)buta-1,3-dien-2-yl)malonate (3ga)}<smiles>C=C(C(=C)C(C)(OCC)C(=O)OCC)c1ccc(C(F)(F)F)cc1</smiles>

The title compound was prepared according to GP5 and isolated as a yellow oil (31.2 mg, $0.084 \mathrm{mmol}, 56 \%) .{ }^{1} \mathrm{H}$ NMR (400 MHz, Chloroform- $\left.d\right) \delta 7.60-7.46$ (m, $4 \mathrm{H}), 5.50(\mathrm{~s}, 1 \mathrm{H}), 5.45(\mathrm{~d}, J=1.1 \mathrm{~Hz}, 1 \mathrm{H}), 5.34(\mathrm{~d}, J=1.1 \mathrm{~Hz}, 1 \mathrm{H}), 5.33(\mathrm{~s}, 1 \mathrm{H})$, 4.10 (qq, $J=10.8,7.1 \mathrm{~Hz}, 4 \mathrm{H}), 1.55(\mathrm{~s}, 3 \mathrm{H}), 1.22$ (t, $J=7.1 \mathrm{~Hz}, 6 \mathrm{H}) .{ }^{13} \mathrm{C} \mathrm{NMR}(101 \mathrm{MHz}$, Chloroform- $d) \delta 171.1 \times$ 2, 147.8, 146.0, 144.4 (q, $J=1.3 \mathrm{~Hz}), 129.7$ (q, $J=32.4 \mathrm{~Hz}), 127.7 \times 2,125.2 \times 2$ (q, J = 4.1 Hz), 124.3 (q, J= 272.1 Hz), 121.3, 117.9, $61.7 \times 2,59.4,22.3,14.0 \times 2 .{ }^{19} \mathrm{~F}$ NMR (376 MHz, Chloroform-d) $\delta-62.38 . \mathrm{HRMS}(\mathrm{ESI})$ $\mathrm{m} / \mathrm{z}:[\mathrm{M}+\mathrm{H}]^{+}$Calcd for $\mathrm{C}_{19} \mathrm{H}_{22} \mathrm{~F}_{3} \mathrm{O}_{4} 371.1465$; Found: 371.1470 .

Diethyl 2-methyl-2-(3-(4-nitrophenyl)buta-1,3-dien-2-yl)malonate (3ha) 
$\mathrm{COO}_{\mathrm{CO}_{2} \mathrm{Et}}^{\mathrm{Me}}$

The title compound was prepared according to GP5 and isolated as a yellow oil (19.9 mg, $0.057 \mathrm{mmol}, 38 \%) .{ }^{1} \mathrm{H}$ NMR (400 MHz, Chloroform- $\left.d\right) \delta 8.19-8.14$ (m, 2H), $7.65-7.54(\mathrm{~m}, 2 \mathrm{H}), 5.55-5.52(\mathrm{~m}, 2 \mathrm{H}), 5.43(\mathrm{~d}, J=0.9 \mathrm{~Hz}, 1 \mathrm{H}), 5.34(\mathrm{~s}$, $1 \mathrm{H}), 4.20-4.00(\mathrm{~m}, 4 \mathrm{H}), 1.56(\mathrm{~s}, 3 \mathrm{H}), 1.22(\mathrm{t}, J=7.1 \mathrm{~Hz}, 6 \mathrm{H}) .{ }^{13} \mathrm{C}$ NMR $(101 \mathrm{MHz}$, Chloroform- $d) \delta 170.8 \times 2$, $147.3,147.1,147.1,145.5,128.0 \times 2,123.4 \times 2,121.7,119.2,61.7 \times 2,59.3,22.2,13.9 \times 2 . H R M S(E S I) m / z:[M$ $+\mathrm{H}]^{+}$Calcd for $\mathrm{C}_{18} \mathrm{H}_{22} \mathrm{NO}_{6}$ 348.1442; Found: 348.1447.

\section{Diethyl 2-(3-(4-(ethoxycarbonyl)phenyl)buta-1,3-dien-2-yl)-2-methylmalonate (3ia)}<smiles>C=C(C(=C)C(C)(C)OCC)c1ccc(C(=O)OCC)cc1</smiles>

The title compound was prepared according to GP5 and isolated as a yellow oil (35.9 mg, $0.096 \mathrm{mmol}, 64 \%) .{ }^{1} \mathrm{H}$ NMR $(400 \mathrm{MHz}$, Chloroform- $d$ ) $\delta 7.97$ (d, $J=$ $8.4 \mathrm{~Hz}, 2 \mathrm{H}), 7.46(\mathrm{~d}, J=8.4 \mathrm{~Hz}, 2 \mathrm{H}), 5.51-5.46(\mathrm{~m}, 2 \mathrm{H}), 5.34(\mathrm{~s}, 2 \mathrm{H}), 4.37$ (q, $J=7.1 \mathrm{~Hz}, 2 \mathrm{H}), 4.17-4.04(\mathrm{~m}, 4 \mathrm{H}), 1.53(\mathrm{~s}, 3 \mathrm{H}), 1.39(\mathrm{t}, J=7.1 \mathrm{~Hz}, 3 \mathrm{H}), 1.22$ $(\mathrm{t}, J=7.1 \mathrm{~Hz}, 6 \mathrm{H}) .{ }^{13} \mathrm{C}$ NMR $(101 \mathrm{MHz}$, Chloroform- $d) \delta 171.0 \times 2,166.4,148.1,145.9,145.1,129.5,129.4 \times 2$, $127.1 \times 2,121.0,117.7,61.6 \times 2,60.9,59.3,22.0,14.3,13.9 \times 2 . \mathrm{HRMS}(\mathrm{ESI}) \mathrm{m} / \mathrm{z}:[\mathrm{M}+\mathrm{H}]^{+}$Calcd for $\mathrm{C}_{21} \mathrm{H}_{27} \mathrm{O}_{6}$ 375.1802; Found: 375.1808 .

\section{Diethyl 2-(3-(3-bromophenyl)buta-1,3-dien-2-yl)-2-methylmalonate (3ja)}<smiles>C=C(C(=C)C(C)(OCC)OCC)c1cccc(Br)c1</smiles>

The title compound was prepared according to GP5 and isolated as a yellow oil (31.9 mg, $0.084 \mathrm{mmol}, 56 \%) .{ }^{1} \mathrm{H} \mathrm{NMR}(400 \mathrm{MHz}$, Chloroform- $d) \delta 7.53$ (t, $\left.J=1.9 \mathrm{~Hz}, 1 \mathrm{H}\right)$, 7.39 (ddd, $J=7.9,2.0,1.1 \mathrm{~Hz}, 1 \mathrm{H}), 7.32(\mathrm{dt}, J=7.8,1.3 \mathrm{~Hz}, 1 \mathrm{H}), 7.17(\mathrm{t}, J=7.9 \mathrm{~Hz}$, 1H), $5.47(\mathrm{~s}, 1 \mathrm{H}), 5.39(\mathrm{~d}, J=1.2 \mathrm{~Hz}, 1 \mathrm{H}), 5.33(\mathrm{~s}, 1 \mathrm{H}), 5.27(\mathrm{~d}, J=1.2 \mathrm{~Hz}, 1 \mathrm{H}), 4.23-3.96(\mathrm{~m}, 4 \mathrm{H}), 1.54(\mathrm{~s}, 3 \mathrm{H})$, $1.23(\mathrm{t}, J=7.1 \mathrm{~Hz}, 6 \mathrm{H}) .{ }^{13} \mathrm{C} N \mathrm{NMR}(101 \mathrm{MHz}$, Chloroform- $d) \delta 171.0 \times 2,147.6,145.9,142.8,130.6,130.2,129.6$, 125.9, 122.2, 121.0, 117.2, $61.6 \times 2,59.2,22.1,13.9 \times 2$. HRMS $(E S I) ~ m / z:[M+H]^{+}$Calcd for $\mathrm{C}_{18} \mathrm{H}_{22}{ }^{79} \mathrm{BrO}_{4}$ 381.0696; Found: 381.0702.

\section{Diethyl 2-(3-(2-fluorophenyl)buta-1,3-dien-2-yl)-2-methylmalonate (3ka)}<smiles>C=C(C(=C)C(C)(OCC)C(=O)OCC)c1ccccc1F</smiles>

The title compound was prepared according to modified GP5 as the reaction was carried out at $80{ }^{\circ} \mathrm{C}$ and isolated as a yellow oil $(29.4 \mathrm{mg}, 0.092 \mathrm{mmol}, 61 \%) .{ }^{1} \mathrm{H} \mathrm{NMR}(400 \mathrm{MHz}$, Chloroform-d) $\delta 7.26(\mathrm{~d}, J=1.8 \mathrm{~Hz}, 1 \mathrm{H}), 7.24-7.19(\mathrm{~m}, 1 \mathrm{H}), 7.07(\mathrm{dd}, J=7.5,1.2 \mathrm{~Hz}$, 1H), $7.05-6.98(\mathrm{~m}, 1 \mathrm{H}), 5.40(\mathrm{~d}, J=1.0 \mathrm{~Hz}, 1 \mathrm{H}), 5.34(\mathrm{~d}, J=1.3 \mathrm{~Hz}, 1 \mathrm{H}), 5.31(\mathrm{~s}, 1 \mathrm{H}), 5.23(\mathrm{~s}, 1 \mathrm{H}), 4.21-4.05$ (m, 4H), $1.62(\mathrm{~s}, 3 \mathrm{H}), 1.22(\mathrm{t}, J=7.1 \mathrm{~Hz}, 6 \mathrm{H}) .{ }^{13} \mathrm{C}$ NMR (101 MHz, Chloroform- $\left.d\right) \delta 171.1 \times 2,159.8(\mathrm{~d}, J=248.8$ $\mathrm{Hz}), 146.3,142.4,131.0(\mathrm{~d}, J=3.5 \mathrm{~Hz}), 129.1(\mathrm{~d}, J=8.2 \mathrm{~Hz}), 128.8(\mathrm{~d}, J=13.6 \mathrm{~Hz}), 123.6(\mathrm{~d}, J=3.8 \mathrm{~Hz}), 119.8$, $119.6(\mathrm{~d}, J=3.4 \mathrm{~Hz}), 115.7(\mathrm{~d}, J=22.2 \mathrm{~Hz}), 61.6 \times 2,59.1,21.9,13.8 \times 2 .{ }^{19} \mathrm{~F}$ NMR $(376 \mathrm{MHz}$, Chloroform- $d) \delta-$ 114.05. HRMS (ESI) m/z: $[\mathrm{M}+\mathrm{H}]^{+}$Calcd for $\mathrm{C}_{18} \mathrm{H}_{22} \mathrm{FO}_{4}$ 321.1497; Found: 321.1502. 


\section{Diethyl 2-methyl-2-(3-(o-tolyl)buta-1,3-dien-2-yl)malonate (3la)}<smiles>C=C(C(=O)C(C)(OCC)C(=O)OCC)c1ccccc1C</smiles>

The title compound was prepared according to modified GP5 as the reaction was carried out at $80^{\circ} \mathrm{C}$ and isolated as a yellow oil $(16.1 \mathrm{mg}, 0.051 \mathrm{mmol}, 34 \%) .{ }^{1} \mathrm{H} \mathrm{NMR}(400 \mathrm{MHz}$, Chloroform-d) $\delta 7.23-7.00(\mathrm{~m}, 4 \mathrm{H}), 5.30(\mathrm{~s}, 1 \mathrm{H}), 5.18(\mathrm{~d}, J=1.1 \mathrm{~Hz}, 1 \mathrm{H}), 5.06(\mathrm{~s}, 1 \mathrm{H})$, $4.93(\mathrm{~s}, 1 \mathrm{H}), 4.22-4.13(\mathrm{~m}, 4 \mathrm{H}), 2.24(\mathrm{~s}, 3 \mathrm{H}), 1.75(\mathrm{~s}, 3 \mathrm{H}), 1.26(\mathrm{t}, \mathrm{J}=7.1 \mathrm{~Hz}, 6 \mathrm{H}) .{ }^{13} \mathrm{C}$ NMR $(101 \mathrm{MHz}$, Chloroformd) $\delta 171.3 \times 2,147.1,146.0,141.6,136.1,129.8,129.7,127.2,125.3,119.5,117.1,61.6 \times 2,59.1,22.3,19.7$, $13.9 \times 2 . \mathrm{HRMS}(\mathrm{ESI}) \mathrm{m} / \mathrm{z}:[\mathrm{M}+\mathrm{H}]^{+}$Calcd for $\mathrm{C}_{19} \mathrm{H}_{25} \mathrm{O}_{4}$ 317.1747; Found: 317.1753 .

\section{Diethyl 2-methyl-2-(3-(naphthalen-2-yl)buta-1,3-dien-2-yl)malonate (3ma)}<smiles>C=C(C(=C)C(C)(COCC)C(=O)OCC)c1ccc2ccccc2c1</smiles>

The title compound was prepared according to GP5 and isolated as a yellow oil (36.9 mg, $0.105 \mathrm{mmol}, 70 \%) .{ }^{1} \mathrm{H}$ NMR (400 MHz, Chloroform- $\left.d\right) \delta 7.85-7.76$ (m, 4H), $7.56(\mathrm{dd}, J=8.6,1.8 \mathrm{~Hz}, 1 \mathrm{H}), 7.49-7.43(\mathrm{~m}, 2 \mathrm{H}), 5.53(\mathrm{~s}, 2 \mathrm{H}), 5.42(\mathrm{~s}, 1 \mathrm{H})$, $5.34(\mathrm{~d}, J=1.2 \mathrm{~Hz}, 1 \mathrm{H}), 4.19-4.00(\mathrm{~m}, 4 \mathrm{H}), 1.57(\mathrm{~s}, 3 \mathrm{H}), 1.21(\mathrm{t}, J=7.1 \mathrm{~Hz}, 6 \mathrm{H}) .{ }^{13} \mathrm{C}$ NMR $(101 \mathrm{MHz}$, Chloroformd) $\delta 171.2 \times 2,148.7,146.3,137.9,133.1,132.8,128.2,127.7,127.5,126.4,126.1,126.0,125.3,120.8,116.5$, $61.5 \times 2,59.4,22.0,13.8 \times 2 . \mathrm{HRMS}(\mathrm{ESI}) \mathrm{m} / \mathrm{z}:[\mathrm{M}+\mathrm{H}]^{+}$Calcd for $\mathrm{C}_{22} \mathrm{H}_{25} \mathrm{O}_{4}$ 353.1747; Found: 353.1753.

\section{Diethyl 2-methyl-2-(3-(thiophen-3-yl)buta-1,3-dien-2-yl)malonate (3na)}<smiles>C=C(C(=C)C(C)(C)C(=O)OCC)c1ccsc1</smiles>

The title compound was prepared according to GP5 and isolated as a yellow oil (23.2 $\mathrm{mg}$, $0.075 \mathrm{mmol}, 50 \%) .{ }^{1} \mathrm{H}$ NMR (400 MHz, Chloroform- $d$ ) $\delta 7.25$ (dd, $\left.J=5.0,3.1 \mathrm{~Hz}, 1 \mathrm{H}\right)$, 7.20 (dd, $J=3.0,1.3 \mathrm{~Hz}, 1 \mathrm{H}), 7.18(\mathrm{dd}, J=4.9,1.4 \mathrm{~Hz}, 1 \mathrm{H}), 5.45-5.43(\mathrm{~m}, 2 \mathrm{H}), 5.37$ (d, $J=0.6 \mathrm{~Hz}, 1 \mathrm{H}), 5.14(\mathrm{~d}, J=1.4 \mathrm{~Hz}, 1 \mathrm{H}), 4.19-4.08(\mathrm{~m}, 4 \mathrm{H}), 1.56(\mathrm{~s}, 3 \mathrm{H}), 1.23(\mathrm{t}, J=7.1 \mathrm{~Hz}, 6 \mathrm{H}) .{ }^{13} \mathrm{C}$ NMR $(101$ $\mathrm{MHz}$, Chloroform-d) $\delta 171.3 \times 2,146.3,143.2,142.4,126.2,125.7,123.0,120.0,114.4,61.7 \times 2,59.5,21.8,14.0$ $\times$ 2. HRMS $(E S I) m / z:[M+H]+$ Calcd for $\mathrm{C}_{16} \mathrm{H}_{21} \mathrm{O}_{4} \mathrm{~S}$ 309.1155; Found: 309.1161.

\section{Diethyl 2-methyl-2-(2-phenylhepta-1,3-dien-3-yl)malonate (3oa)}<smiles>C=C(C(=C[PH+])C(=C)C(C)(OCC)OCC)c1ccccc1</smiles>

The title compound was prepared according to GP5 and isolated as a yellow oil (34.8 $\mathrm{mg}, 0.100 \mathrm{mmol}, 67 \%, E / Z=9.1: 1) .{ }^{1} \mathrm{H}$ NMR $(400 \mathrm{MHz}$, Chloroform- $d) \delta 7.46-7.42(\mathrm{~m}$, 2H), $7.34-7.27(\mathrm{~m}, 3 \mathrm{H}), 5.83(\mathrm{t}, J=7.3 \mathrm{~Hz}, 1 \mathrm{H}), 5.69$ (d, J = 1.7 Hz, 1H), 5.12 (d, J= $1.6 \mathrm{~Hz}, 1 \mathrm{H}), 4.13-4.05(\mathrm{~m}, 4 \mathrm{H}), 2.05-1.99(\mathrm{~m}, 2 \mathrm{H}), 1.43(\mathrm{~s}, 3 \mathrm{H}), 1.38$ (dd, $J=14.6$, 7.3 Hz, 2H), 1.21 (d, J=7.1 Hz, 6H), 0.87 (t, J=7.4 Hz, 3H). ${ }^{13} \mathrm{C}$ NMR $(101 \mathrm{MHz}$, Chloroform- $d) \delta 171.8 \times 2,145.1$, $139.6,136.4,134.6,128.3 \times 2,127.7,126.3 \times 2,116.3,61.4 \times 2,59.9,31.9,22.7,21.7,14.0,13.9 \times 2 . H R M S$ (ESI) m/z: $[\mathrm{M}+\mathrm{H}]^{+}$Calcd for $\mathrm{C}_{21} \mathrm{H}_{29} \mathrm{O}_{4}$ 345.2060; Found: 345.2066.

\section{Diethyl 2-methyl-2-(2-phenylocta-1,3-dien-3-yl)malonate (3pa)}




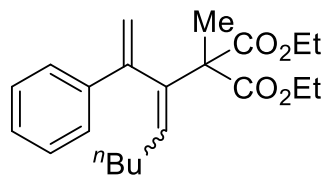

The title compound was prepared according to GP5 and isolated as a yellow oil (34.1 $\mathrm{mg}, 0.095 \mathrm{mmol}, 63 \%, E / Z=10: 1) .{ }^{1} \mathrm{H} \mathrm{NMR}(400 \mathrm{MHz}$, Chloroform- $d) \delta 7.49-7.41(\mathrm{~m}$, 2H), $7.33-7.28(\mathrm{~m}, 3 \mathrm{H}), 5.82(\mathrm{t}, J=7.3 \mathrm{~Hz}, 1 \mathrm{H}), 5.69(\mathrm{~d}, J=1.6 \mathrm{~Hz}, 1 \mathrm{H}), 5.12(\mathrm{~d}, J=$ $1.5 \mathrm{~Hz}, 1 \mathrm{H}), 4.13-4.06(\mathrm{~m}, 4 \mathrm{H}), 2.07-2.02(\mathrm{~m}, 2 \mathrm{H}), 1.43(\mathrm{~s}, 3 \mathrm{H}), 1.38-1.28(\mathrm{~m}, 4 \mathrm{H})$, $1.22(\mathrm{~d}, J=7.1 \mathrm{~Hz}, 6 \mathrm{H}), 0.84$ (t, $J=7.1 \mathrm{~Hz}, 3 \mathrm{H}) .{ }^{13} \mathrm{C} \mathrm{NMR}(101 \mathrm{MHz}$, Chloroform- $d) \delta 171.7 \times 2,144.9,139.5$, 136.0, 134.6, $128.2 \times 2,127.6,126.2 \times 2,116.2,61.3 \times 2,59.7,31.6,29.5,22.3,21.5,14.0,13.9 \times 2 . \mathrm{HRMS}(\mathrm{ESI})$ $\mathrm{m} / \mathrm{z}:[\mathrm{M}+\mathrm{H}]^{+}$Calcd for $\mathrm{C}_{22} \mathrm{H}_{31} \mathrm{O}_{4}$ 359.2217; Found: 359.2223 .

\section{Diethyl 2-(2,5-diphenylpenta-1,3-dien-3-yl)-2-methylmalonate (3qa)}

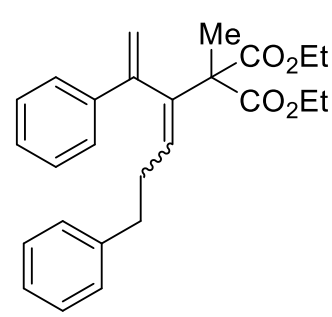

The title compound was prepared according to GP5 and isolated as a yellow oil (33.7 $\mathrm{mg}, 0.083 \mathrm{mmol}, 55 \%, E / Z=10: 1) .{ }^{1} \mathrm{H}$ NMR $(400 \mathrm{MHz}$, Chloroform- $d) \delta 7.38-7.34(\mathrm{~m}$, 2H), $7.29(\mathrm{dd}, J=2.6,1.8 \mathrm{~Hz}, 1 \mathrm{H}), 7.24-7.22(\mathrm{~m}, 3 \mathrm{H}), 7.19-7.13(\mathrm{~m}, 2 \mathrm{H}), 7.11-7.08$ (m, 2H), $5.88(\mathrm{t}, J=7.3 \mathrm{~Hz}, 1 \mathrm{H}), 5.65(\mathrm{~d}, J=1.6 \mathrm{~Hz}, 1 \mathrm{H}), 5.02(\mathrm{~d}, J=1.5 \mathrm{~Hz}, 1 \mathrm{H}), 4.07$ (ddd, $J=10.7,7.1,3.5 \mathrm{~Hz}, 4 \mathrm{H}$ ), 2.67 (dd, $J=8.6,6.8 \mathrm{~Hz}, 2 \mathrm{H}$ ), $2.39-2.34(\mathrm{~m}, 2 \mathrm{H}), 1.42$ $(\mathrm{s}, 3 \mathrm{H}), 1.20(\mathrm{~d}, J=7.1 \mathrm{~Hz}, 6 \mathrm{H}) .{ }^{13} \mathrm{C}$ NMR $(101 \mathrm{MHz}$, Chloroform- $d) \delta 171.5 \times 2,144.7$, 141.6, 139.3, 137.0, 133.2, $128.5 \times 2,128.3,128.2 \times 2,128.2 \times 2,127.6,126.1 \times 2,125.8,61.3 \times 2,59.6,35.7$, 31.9, 21.5, $13.9 \times 2$. HRMS (ESI) m/z: [M + H] Calcd for $\mathrm{C}_{26} \mathrm{H}_{31} \mathrm{O}_{4}$ 407.2217; Found: 407.2222.

\section{Dimethyl 2-methyl-2-(3-phenylbuta-1,3-dien-2-yl)malonate (3ab)}<smiles>C=C(C(=C)C(C)(O)OC(C)=O)C(C)(C)OC(C)=O</smiles>

The title compound was prepared according to GP5 and isolated as a yellow oil $(26.4 \mathrm{mg}$, $0.096 \mathrm{mmol}, 64 \%) .{ }^{1} \mathrm{H}$ NMR (400 MHz, Chloroform- $d$ ) $\delta 7.41-7.33(\mathrm{~m}, 2 \mathrm{H}), 7.32-7.25$ (m, 3H), $5.41(\mathrm{~s}, 1 \mathrm{H}), 5.37$ (d, J=1.2 Hz, 1H), 5.34 (s, 1H), $5.20(\mathrm{~d}, J=1.2 \mathrm{~Hz}, 1 \mathrm{H}), 3.62$ $(\mathrm{s}, 6 \mathrm{H}), 1.54(\mathrm{~s}, 3 \mathrm{H}) .{ }^{13} \mathrm{C}$ NMR $(101 \mathrm{MHz}$, Chloroform-d) $\delta 171.5 \times 2,148.7,146.2,140.4,128.1 \times 2,127.7,127.2$ $\times 2,120.5,116.3,59.4 \times 2,52.6,21.9 . \mathrm{HRMS}(\mathrm{ESI}) \mathrm{m} / \mathrm{z}:[\mathrm{M}+\mathrm{H}]^{+}$Calcd for $\mathrm{C}_{16} \mathrm{H}_{19} \mathrm{O}_{4}$ 275.1278; Found: 275.1283 .

\section{Diethyl 2-ethyl-2-(3-phenylbuta-1,3-dien-2-yl)malonate (3ac)}<smiles>C=C(C(=C)C(CC)(COCC)COCC)c1ccccc1</smiles>
The title compound was prepared according to modified GP5 as the reaction was carried out at $70{ }^{\circ} \mathrm{C}$ and isolated as a yellow oil $(31.3 \mathrm{mg}, 0.099 \mathrm{mmol}, 66 \%) .{ }^{1} \mathrm{H} \mathrm{NMR}(400 \mathrm{MHz}$, Chloroform- $d$ ) $\delta 7.40-7.34(\mathrm{~m}, 2 \mathrm{H}), 7.31-7.25(\mathrm{~m}, 3 \mathrm{H}), 5.60(\mathrm{~d}, J=0.9 \mathrm{~Hz}, 1 \mathrm{H}), 5.35(\mathrm{~d}$, $J=0.9 \mathrm{~Hz}, 1 \mathrm{H}), 5.30(\mathrm{~d}, J=1.3 \mathrm{~Hz}, 1 \mathrm{H}), 5.19(\mathrm{~d}, J=1.4 \mathrm{~Hz}, 1 \mathrm{H}), 4.10-3.94(\mathrm{~m}, 4 \mathrm{H}), 2.02(\mathrm{q}, J=7.4 \mathrm{~Hz}, 2 \mathrm{H})$, $1.18(\mathrm{t}, J=7.1 \mathrm{~Hz}, 6 \mathrm{H}), 0.90(\mathrm{t}, J=7.4 \mathrm{~Hz}, 3 \mathrm{H}) \cdot{ }^{13} \mathrm{C}$ NMR $(101 \mathrm{MHz}$, Chloroform- $d) \delta 170.1 \times 2,149.3,145.0$, $140.7,127.9 \times 2,127.6,127.3 \times 2,121.3,115.9,63.3,61.1 \times 2,28.4,13.9 \times 2,9.6 . \mathrm{HRMS}(\mathrm{ESI}) \mathrm{m} / \mathrm{z}:[\mathrm{M}+\mathrm{H}]^{+}$ Calcd for $\mathrm{C}_{19} \mathrm{H}_{25} \mathrm{O}_{4}$ 317.1747; Found: 317.1753 . 
${ }_{\mathrm{CO}_{2} \mathrm{Et}}^{\mathrm{Ph}}$

The title compound was prepared according to GP5 and isolated as a yellow oil $(35.1 \mathrm{mg}$, $0.093 \mathrm{mmol}, 62 \%) .{ }^{1} \mathrm{H}$ NMR $(400 \mathrm{MHz}$, Chloroform- $d) \delta 7.45-7.39(\mathrm{~m}, 2 \mathrm{H}), 7.34-7.27(\mathrm{~m}$, $3 \mathrm{H}), 7.23-7.17(\mathrm{~m}, 5 \mathrm{H}), 5.41(\mathrm{~s}, 1 \mathrm{H}), 5.38(\mathrm{~d}, J=1.2 \mathrm{~Hz}, 1 \mathrm{H}), 5.34(\mathrm{~s}, 1 \mathrm{H}), 5.25$ (d, J = 1.2 $\mathrm{Hz}, 1 \mathrm{H}), 4.03-3.84(\mathrm{~m}, 4 \mathrm{H}), 3.40(\mathrm{~s}, 2 \mathrm{H}), 1.10(\mathrm{t}, J=7.2 \mathrm{~Hz}, 6 \mathrm{H}) \cdot{ }^{13} \mathrm{C}$ NMR $(101 \mathrm{MHz}$, Chloroform-d) $\delta 169.6 \times 2$, $149.4,144.1,140.7,136.6,130.6 \times 2,127.9 \times 2,127.8 \times 2,127.6,127.5 \times 2,126.8,122.3,115.9,64.4,61.2 \times 2$, 41.3, $13.7 \times 2 . \mathrm{HRMS}(E S I) \mathrm{m} / \mathrm{z}:[\mathrm{M}+\mathrm{H}]^{+}$Calcd for $\mathrm{C}_{24} \mathrm{H}_{27} \mathrm{O}_{4} 379.1904$; Found: 379.1909 .

\section{Diethyl 2-(2-oxopropyl)-2-(3-phenylbuta-1,3-dien-2-yl)malonate (3ae)}<smiles>C=CCC(OCC)(C(=C)C(=C)c1ccccc1)C(=O)OCC</smiles>

The title compound was prepared according to GP5 and isolated as a yellow oil $(32.1 \mathrm{mg}$, $0.093 \mathrm{mmol}, 62 \%) .{ }^{1} \mathrm{H}$ NMR (400 MHz, Chloroform- $d$ ) $\delta 7.41-7.35(\mathrm{~m}, 2 \mathrm{H}), 7.34-7.28(\mathrm{~m}$, $3 \mathrm{H}), 5.50(\mathrm{~s}, 1 \mathrm{H}), 5.40(\mathrm{~d}, J=1.5 \mathrm{~Hz}, 1 \mathrm{H}), 5.35(\mathrm{~s}, 1 \mathrm{H}), 5.17(\mathrm{~d}, J=1.4 \mathrm{~Hz}, 1 \mathrm{H}), 4.16-4.00$ (m, 4H), 3.06 (s, 2H), 2.06 (s, 3H), 1.21 (t, J=7.2 Hz, 6H). ${ }^{13} \mathrm{C}$ NMR (101 MHz, Chloroform-d) $\delta 204.8,169.2 \times 2$, 148.7, 145.0, 139.6, $128.2 \times 2,128.0,127.0 \times 2,120.8,116.7,61.7 \times 2,60.6,48.2,29.9,13.8 \times 2$. HRMS (ESI) $\mathrm{m} / \mathrm{z}:[\mathrm{M}+\mathrm{H}]^{+}$Calcd for $\mathrm{C}_{20} \mathrm{H}_{25} \mathrm{O}_{5}$ 345.1697; Found: 345.1702 .

\section{Diethyl 2-fluoro-2-(3-phenylbuta-1,3-dien-2-yl)malonate (3af)}<smiles>C=C(C(=C)C(F)(C(=O)OCC)C(=O)OCC)c1ccccc1</smiles>

The title compound was prepared according to GP5 and isolated as a yellow oil $(29.4 \mathrm{mg}$, $0.096 \mathrm{mmol}, 64 \%) .{ }^{1} \mathrm{H}$ NMR (400 MHz, Chloroform- $d$ ) $\delta 7.40-7.22(\mathrm{~m}, 5 \mathrm{H}), 5.57$ (d, J = 3.0 $\mathrm{Hz}, 1 \mathrm{H}), 5.48-5.40(\mathrm{~m}, 2 \mathrm{H}), 5.35(\mathrm{~d}, J=2.0 \mathrm{~Hz}, 1 \mathrm{H}), 4.30-4.15(\mathrm{~m}, 4 \mathrm{H}), 1.27(\mathrm{t}, J=7.1$ $\mathrm{Hz}, 6 \mathrm{H}) .{ }^{13} \mathrm{C}$ NMR $(101 \mathrm{MHz}$, Chloroform- $d) \delta 165.4 \times 2$ (d, $\left.J=26.9 \mathrm{~Hz}\right), 146.1,142.1$ (d, $\left.J=19.7 \mathrm{~Hz}\right), 140.4$, $128.1 \times 2,127.9 \times 2,127.8,123.1(\mathrm{~d}, J=7.2 \mathrm{~Hz}), 116.8(\mathrm{~d}, J=2.4 \mathrm{~Hz}), 95.1$ (d, J = 199.4 Hz), $62.8 \times 2,13.8 \times 2$. ${ }^{19} \mathrm{~F}$ NMR (376 MHz, Chloroform- $d$ ) $\delta$-150.84. HRMS (ESI) m/z: $[\mathrm{M}+\mathrm{H}]^{+}$Calcd for $\mathrm{C}_{17} \mathrm{H}_{20} \mathrm{FO}_{4}$ 307.1340; Found: 307.1342 .

\section{Diethyl 2-(cyclopropylmethyl)-2-(3-phenylbuta-1,3-dien-2-yl)malonate (3ag)}

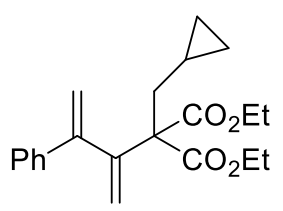

The title compound was prepared according to GP5 and isolated as a yellow oil $(30.5 \mathrm{mg}$, $0.089 \mathrm{mmol}, 59 \%) .{ }^{1} \mathrm{H}$ NMR $(400 \mathrm{MHz}$, Chloroform- $d) \delta 7.40-7.34(\mathrm{~m}, 2 \mathrm{H}), 7.30-7.25(\mathrm{~m}$, 3H), $5.69(\mathrm{~d}, J=0.9 \mathrm{~Hz}, 1 \mathrm{H}), 5.36(\mathrm{~d}, J=1.1 \mathrm{~Hz}, 1 \mathrm{H}), 5.31(\mathrm{~d}, J=1.4 \mathrm{~Hz}, 1 \mathrm{H}), 5.21(\mathrm{~d}, J=$ $1.4 \mathrm{~Hz}, 1 \mathrm{H}), 4.13-3.89(\mathrm{~m}, 4 \mathrm{H}), 1.94(\mathrm{~d}, J=6.6 \mathrm{~Hz}, 2 \mathrm{H}), 1.18(\mathrm{t}, J=7.1 \mathrm{~Hz}, 6 \mathrm{H}), 0.90-$ $0.78(\mathrm{~m}, 1 \mathrm{H}), 0.42-0.36(\mathrm{~m}, 2 \mathrm{H}), 0.04--0.01(\mathrm{~m}, 2 \mathrm{H}) .{ }^{13} \mathrm{C}$ NMR $(101 \mathrm{MHz}$, Chloroform-d) $\delta 170.2 \times 2,149.4$, $145.3,140.7,127.9 \times 2,127.6,127.3 \times 2,121.4,115.9,62.7,61.1 \times 2,40.3,13.8 \times 2,6.9,4.6 \times 2 . H R M S(E S I)$ $\mathrm{m} / \mathrm{z}:[\mathrm{M}+\mathrm{H}]^{+}$Calcd for $\mathrm{C}_{21} \mathrm{H}_{27} \mathrm{O}_{4}$ 343.1904; Found: 343.1909 .

\section{Diethyl 2-(cyclopentylmethyl)-2-(3-phenylbuta-1,3-dien-2-yl)malonate (3ah)}

The title compound was prepared according to modified GP5 as the reaction was carried out at $80^{\circ} \mathrm{C}$ and isolated 
<smiles>C=C(C(=C)C(COCC)(CC1CCCC1)C(=O)OCC)c1ccccc1</smiles>

as a yellow oil (36.5 mg, $0.099 \mathrm{mmol}, 66 \%) .{ }^{1} \mathrm{H}$ NMR $(400 \mathrm{MHz}$, Chloroform- $d) \delta 7.39-7.34$ (m, 2H), $7.30-7.22(\mathrm{~m}, 3 \mathrm{H}), 5.73(\mathrm{~d}, J=0.9 \mathrm{~Hz}, 1 \mathrm{H}), 5.35(\mathrm{~d}, J=0.9 \mathrm{~Hz}, 1 \mathrm{H}), 5.29(\mathrm{~d}, J=$ $1.4 \mathrm{~Hz}, 1 \mathrm{H}), 5.19(\mathrm{~d}, J=1.4 \mathrm{~Hz}, 1 \mathrm{H}), 4.08-3.88(\mathrm{~m}, 4 \mathrm{H}), 1.93(\mathrm{~d}, J=5.5 \mathrm{~Hz}, 2 \mathrm{H}), 1.64-$ $1.57(\mathrm{~m}, 4 \mathrm{H}), 1.16(\mathrm{t}, J=7.2 \mathrm{~Hz}, 6 \mathrm{H}), 1.13-1.02(\mathrm{~m}, 1 \mathrm{H}), 0.94-0.85(\mathrm{~m}, 2 \mathrm{H}) .{ }^{13} \mathrm{C}$ NMR $(101 \mathrm{MHz}$, Chloroform-d) $\delta 170.4 \times 2,149.6,145.3,140.9,128.0 \times 2,127.6,127.5 \times 2,121.5,116.1,61.8 \times 2$, 61.1, 42.3, $34.4 \times 2$ 2, 34.3, 26.5, 26.3, $13.9 \times 2 . \mathrm{HRMS}(\mathrm{ESI}) \mathrm{m} / \mathrm{z}:[\mathrm{M}+\mathrm{H}]^{+}$Calcd for $\mathrm{C}_{23} \mathrm{H}_{31} \mathrm{O}_{4} 371.2217$; Found: 371.2223.

\section{Triethyl 5-methylene-6-phenylhept-6-ene-1,4,4-tricarboxylate (3ai)}<smiles>C=C(C(=C)C(C)(CCCC(=O)OCC)C(=O)OCC)c1ccccc1</smiles>

The title compound was prepared according to GP5 and isolated as a yellow oil (40.0 mg, 0.099 mmol, 66\%). ${ }^{1} \mathrm{H}$ NMR (400 MHz, Chloroform- $\left.d\right) \delta 7.38-7.33(\mathrm{~m}, 2 \mathrm{H}), 7.30$ $-7.25(\mathrm{~m}, 2 \mathrm{H}), 7.25-7.22(\mathrm{~m}, 1 \mathrm{H}), 5.62(\mathrm{~s}, 1 \mathrm{H}), 5.36(\mathrm{~d}, J=0.9 \mathrm{~Hz}, 1 \mathrm{H}), 5.30(\mathrm{~d}, J$ $=1.4 \mathrm{~Hz}, 1 \mathrm{H}), 5.19(\mathrm{~d}, J=1.4 \mathrm{~Hz}, 1 \mathrm{H}), 4.11-4.03(\mathrm{~m}, 4 \mathrm{H}), 4.02-3.94(\mathrm{~m}, 2 \mathrm{H}), 2.21(\mathrm{t}, J=7.5 \mathrm{~Hz}, 2 \mathrm{H}), 2.03-$ $1.91(\mathrm{~m}, 2 \mathrm{H}), 1.68-1.60(\mathrm{~m}, 2 \mathrm{H}), 1.23-1.16(\mathrm{~m}, 9 \mathrm{H}) .{ }^{13} \mathrm{C}$ NMR (101 MHz, Chloroform- $\left.d\right) \delta 173.1,169.9 \times 2$, $149.2,144.7,140.6,127.9 \times 2,127.6,127.3 \times 2,121.6,116.1,62.6,61.3 \times 2,60.2,34.4,34.4,20.6,14.2,13.8 \times$ 2. HRMS (ESI) m/z: [M + H] $]^{+}$Calcd for $\mathrm{C}_{23} \mathrm{H}_{31} \mathrm{O}_{6}$ 403.2115; Found: 403.2121 .

\section{Diethyl 2-(2-((tert-butyldimethylsilyl)oxy)ethyl)-2-(3-phenylbuta-1,3-dien-2-yl)malonate (3aj)}

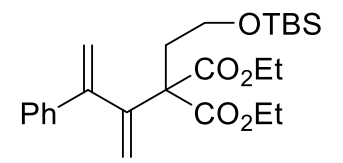

The title compound was prepared according to GP5 and isolated as a yellow oil (46.7 mg, $0.105 \mathrm{mmol}, 70 \%) .{ }^{1} \mathrm{H}$ NMR (400 MHz, Chloroform- $d$ ) $\delta 7.38$ (dd, $J=8.1,1.6 \mathrm{~Hz}$, 2H), $7.32-7.26(\mathrm{~m}, 3 \mathrm{H}), 5.63(\mathrm{~d}, J=0.8 \mathrm{~Hz}, 1 \mathrm{H}), 5.38(\mathrm{~d}, J=0.8 \mathrm{~Hz}, 1 \mathrm{H}), 5.33(\mathrm{~d}, J=$ $1.3 \mathrm{~Hz}, 1 \mathrm{H}), 5.20(\mathrm{~d}, J=1.3 \mathrm{~Hz}, 1 \mathrm{H}), 4.09-3.96(\mathrm{~m}, 4 \mathrm{H}), 3.73-3.65(\mathrm{~m}, 2 \mathrm{H}), 2.34-2.23(\mathrm{~m}, 2 \mathrm{H}), 1.18(\mathrm{t}, J=7.1$ $\mathrm{Hz}, 6 \mathrm{H}), 0.86(\mathrm{~s}, 9 \mathrm{H}), 0.02(\mathrm{~s}, 6 \mathrm{H}) .{ }^{13} \mathrm{C}$ NMR $(101 \mathrm{MHz}$, Chloroform-d) $\delta 169.9 \times 2,149.2,144.7,140.6,127.9 \times 2$, $127.6,127.4 \times 2,121.6,116.0,61.3 \times 2,60.9,59.9,37.7,25.9 \times 3,18.3,13.8 \times 2,-5.3 \times 2 . H R M S(E S I) ~ m / z:[M+$ $\mathrm{H}]^{+}$Calcd for $\mathrm{C}_{25} \mathrm{H}_{39} \mathrm{O}_{5} \mathrm{Si}$ 447.2561; Found: 447.2566.

\section{Ethyl 2-acetyl-2-methyl-3-methylene-4-phenylpent-4-enoate (3ak)}<smiles>C=C(C(=C)C(C)(C)C(C)(C)OCC)c1ccccc1</smiles>
The title compound was prepared according to GP5 and isolated as a yellow oil $(15.5 \mathrm{mg}$, $0.057 \mathrm{mmol}, 38 \%) .{ }^{1} \mathrm{H}$ NMR (400 MHz, Chloroform- $d$ ) $\delta 7.39-7.34(\mathrm{~m}, 2 \mathrm{H}), 7.34-7.27$ (m, 3H), $5.41(\mathrm{~d}, J=0.6 \mathrm{~Hz}, 1 \mathrm{H}), 5.36(\mathrm{~d}, J=1.2 \mathrm{~Hz}, 1 \mathrm{H}), 5.33(\mathrm{~d}, J=0.6 \mathrm{~Hz}, 1 \mathrm{H}), 5.16(\mathrm{~d}, J=$ $1.2 \mathrm{~Hz}, 1 \mathrm{H}), 4.09$ (qd, J = 7.1, 1.9Hz, 2H), $2.29(\mathrm{~s}, 3 \mathrm{H}), 1.44(\mathrm{~s}, 3 \mathrm{H}), 1.25-1.18(\mathrm{~m}, 3 \mathrm{H}) .{ }^{13} \mathrm{C} \mathrm{NMR}(101 \mathrm{MHz}$, Chloroform- $d) \delta 204.8,171.4,149.0,146.6,140.4,128.2 \times 2,127.8,127.3 \times 2,121.3,116.0,65.3,61.5,26.9,20.6$, 13.9. HRMS (ESI) m/z: $[\mathrm{M}+\mathrm{H}]^{+}$Calcd for $\mathrm{C}_{17} \mathrm{H}_{21} \mathrm{O}_{3}$ 273.1485; Found: 273.1491.

Ethyl 2-cyano-2-methyl-3-methylene-4-phenylpent-4-enoate (3al) 
$\overbrace{\mathrm{CN}}^{\mathrm{Me}} \mathrm{CO}_{2} \mathrm{Et}$

The title compound was prepared according to modified GP5 as the reaction was carried out at $70{ }^{\circ} \mathrm{C}$ and isolated as a yellow oil $(23.7 \mathrm{mg}, 0.093 \mathrm{mmol}, 62 \%) .{ }^{1} \mathrm{H} \mathrm{NMR}(400 \mathrm{MHz}$, Chloroform-d) $\delta 7.37-7.27(\mathrm{~m}, 5 \mathrm{H}), 5.77(\mathrm{~s}, 1 \mathrm{H}), 5.43(\mathrm{~d}, J=0.9 \mathrm{~Hz}, 1 \mathrm{H}), 5.42(\mathrm{~s}, 1 \mathrm{H}), 5.32$ (d, $J=0.9 \mathrm{~Hz}, 1 \mathrm{H}), 4.05-3.97(\mathrm{~m}, 1 \mathrm{H}), 3.94-3.86(\mathrm{~m}, 1 \mathrm{H}), 1.72(\mathrm{~s}, 3 \mathrm{H}), 1.20(\mathrm{t}, J=7.2 \mathrm{~Hz}, 3 \mathrm{H}) .{ }^{13} \mathrm{C} \mathrm{NMR}(101$ $\mathrm{MHz}$, Chloroform-d) $\delta 167.4,146.8,143.7,139.4,128.2 \times 2,128.1,127.3 \times 2,120.4,119.2,117.6,63.0,48.2,23.3$, 13.7. HRMS (ESI) $\mathrm{m} / \mathrm{z}:[\mathrm{M}+\mathrm{H}]^{+}$Calcd for $\mathrm{C}_{16} \mathrm{H}_{18} \mathrm{NO}_{2} 256.1332$; Found: 256.1338.

\section{Ethyl 2-methyl-3-methylene-4-phenyl-2-(phenylsulfonyl)pent-4-enoate (3am)}<smiles>C=C(C(=C)C(C)(C)C(=O)OCC)C(=O)Oc1ccccc1</smiles>

The title compound was prepared according to modified GP5 as the reaction was carried out at $50^{\circ} \mathrm{C}$ and isolated as a yellow oil $(34.1 \mathrm{mg}, 0.092 \mathrm{mmol}, 61 \%) .{ }^{1} \mathrm{H} \mathrm{NMR}(400 \mathrm{MHz}$, Chloroform- $d$ ) $\delta 7.97$ (dd, $J=8.4,1.3 \mathrm{~Hz}, 2 \mathrm{H}$ ), $7.69-7.62(\mathrm{~m}, 1 \mathrm{H}), 7.53(\mathrm{dd}, J=8.4,7.1$ $\mathrm{Hz}, 2 \mathrm{H}), 7.26(\mathrm{~s}, 5 \mathrm{H}), 5.97(\mathrm{~d}, J=0.8 \mathrm{~Hz}, 1 \mathrm{H}), 5.58(\mathrm{~d}, J=0.7 \mathrm{~Hz}, 1 \mathrm{H}), 5.32(\mathrm{~d}, J=1.4 \mathrm{~Hz}, 1 \mathrm{H}), 4.93(\mathrm{~d}, J=1.3$ $\mathrm{Hz}, 1 \mathrm{H}), 4.01-3.68(\mathrm{~m}, 2 \mathrm{H}), 1.80(\mathrm{~s}, 3 \mathrm{H}), 1.06(\mathrm{t}, J=7.2 \mathrm{~Hz}, 3 \mathrm{H}) .{ }^{13} \mathrm{C}$ NMR (101 MHz, Chloroform-d) $\delta 167.5$, 148.6, 142.4, 139.5, 136.6, 133.8, $132.0 \times 2,128.2 \times 2,128.1 \times 2,127.9,126.8 \times 2,125.4,116.4,76.5,62.2,20.5$, 13.5. HRMS (ESI) m/z: $[\mathrm{M}+\mathrm{H}]^{+}$Calcd for $\mathrm{C}_{21} \mathrm{H}_{23} \mathrm{O}_{4} \mathrm{~S}$ 371.1312; Found: 371.1317.

\section{2-Methyl-3-methylene-4-phenyl-2-tosylpent-4-enenitrile (3an)}<smiles>C=C(C(=C)C(C)(F)C#N)c1ccccc1</smiles>

The title compound was prepared according to GP5 and isolated as a yellow oil ( $34.1 \mathrm{mg}, 0.100$ mmol, 67\%). ${ }^{1} \mathrm{H}$ NMR (400 MHz, Chloroform- $d$ ) $\delta 7.87(\mathrm{~d}, J=8.1 \mathrm{~Hz}, 2 \mathrm{H}), 7.39$ (d, $J=8.0 \mathrm{~Hz}$, 2H), $7.36-7.26(\mathrm{~m}, 5 \mathrm{H}), 5.83(\mathrm{~s}, 1 \mathrm{H}), 5.64(\mathrm{~s}, 1 \mathrm{H}), 5.55(\mathrm{~s}, 1 \mathrm{H}), 5.21(\mathrm{~s}, 1 \mathrm{H}), 2.48(\mathrm{~s}, 3 \mathrm{H}), 1.66$ (s, 3H). ${ }^{13} \mathrm{C}$ NMR (101 MHz, Chloroform-d) $\delta 146.5,146.2,139.2,139.1,131.3 \times 2,130.6,129.7 \times 2,128.6 \times 2$, 128.3, 126.8, $126.4 \times 2,118.8,117.7,65.7,21.8,20.8$. HRMS (ESI) m/z: $[\mathrm{M}+\mathrm{H}]^{+}$Calcd for $\mathrm{C}_{20} \mathrm{H}_{20} \mathrm{NO}_{2} \mathrm{~S} 338.1209$; Found: 338.1215 .

\section{2-Methyl-2-(3-phenylbuta-1,3-dien-2-yl)malononitrile (3ao)}<smiles>C=C(C(=C)C(C)(C#N)C#N)c1ccccc1</smiles>

The title compound was prepared according to GP5 and isolated as a yellow oil $(23.5 \mathrm{mg}$, $0.113 \mathrm{mmol}, 75 \%)$. ${ }^{1} \mathrm{H}$ NMR (400 MHz, Chloroform- $d$ ) $\delta 7.45-7.32$ (m, 5H), 5.96 (d, $J=0.5$ $\mathrm{Hz}, 1 \mathrm{H}), 5.64(\mathrm{~d}, J=0.6 \mathrm{~Hz}, 1 \mathrm{H}), 5.58(\mathrm{~s}, 1 \mathrm{H}), 5.49(\mathrm{~s}, 1 \mathrm{H}), 1.84(\mathrm{~s}, 3 \mathrm{H}) .{ }^{13} \mathrm{C} \mathrm{NMR}(101 \mathrm{MHz}$, Chloroform-d) $\delta 144.8,140.8,138.3,128.8,128.7 \times 2,127.1 \times 2,121.7,119.5,115.1 \times 2,36.3,26.0$. HRMS (ESI) $\mathrm{m} / \mathrm{z}:[\mathrm{M}+\mathrm{H}]^{+}$Calcd for $\mathrm{C}_{14} \mathrm{H}_{13} \mathrm{~N}_{2} 209.1073$; Found: 209.1079 .

\section{Diethyl 2-(3-(1,3-dioxolan-2-yl)propyl)-2-(3-phenylbuta-1,3-dien-2-yl)malonate (3ap)}<smiles>C=C(C(=C)C(C#N)(C#N)CCC1OCCO1)c1ccccc1</smiles>

The title compound was prepared according to GP5 and isolated as a yellow oil (26.9 $\mathrm{mg}$, $0.091 \mathrm{mmol}, 61 \%) .{ }^{1} \mathrm{H}$ NMR (400 MHz, Chloroform- $\left.d\right) \delta 7.39-7.33(\mathrm{~m}, 5 \mathrm{H}), 5.93(\mathrm{~s}, 1 \mathrm{H})$, $5.59(\mathrm{~s}, 2 \mathrm{H}), 5.45(\mathrm{~s}, 1 \mathrm{H}), 4.87(\mathrm{t}, J=4.0 \mathrm{~Hz}, 1 \mathrm{H}), 3.92-3.75(\mathrm{~m}, 4 \mathrm{H}), 2.17-2.08(\mathrm{~m}$, 
2H), $1.98-1.93(\mathrm{~m}, 2 \mathrm{H}) .{ }^{13} \mathrm{C}$ NMR (101 MHz, Chloroform- $\left.d\right) \delta 145.0,139.8,138.3,128.8,128.6 \times 2,127.2 \times 2$, 122.4, 119.6, $114.1 \times 2,102.2,65.1 \times 2,41.8,32.2,29.7$. HRMS $(E S I) ~ m / z:[M+H]^{+}$Calcd for $\mathrm{C}_{18} \mathrm{H}_{19} \mathrm{~N}_{2} \mathrm{O}_{2} 295.1441$; Found: 295.1447.

\section{Diethyl 2-(5-fluoropentyl)-2-(3-phenylbuta-1,3-dien-2-yl)malonate (3aq)}<smiles>C=C(C(=C)C(C)(C#N)CCCCCF)c1ccccc1</smiles>

The title compound was prepared according to GP5 and isolated as a yellow oil (30.2 mg, $0.107 \mathrm{mmol}, 71 \%) .{ }^{1} \mathrm{H}$ NMR (400 MHz, Chloroform- $\left.d\right) \delta 7.43-7.31(\mathrm{~m}, 5 \mathrm{H}), 5.94$ (s, 1H), 5.59 (s, 2H), $5.43(\mathrm{~d}, J=0.8 \mathrm{~Hz}, 1 \mathrm{H}), 4.40$ (dt, J=47.3, 5. $9 \mathrm{~Hz}, 2 \mathrm{H}), 2.00-$ $1.88(\mathrm{~m}, 2 \mathrm{H}), 1.72-1.58(\mathrm{~m}, 4 \mathrm{H}), 1.42-1.31(\mathrm{~m}, 2 \mathrm{H}) .{ }^{13} \mathrm{C}$ NMR (101 MHz, Chloroform- $\left.d\right) \delta 145.1,139.9,138.3$, $128.8 \times 2,128.6 \times 2,127.1,122.2,119.7,114.3 \times 2,83.5(\mathrm{~d}, J=164.8 \mathrm{~Hz}), 42.3,37.9,29.8(\mathrm{~d}, J=19.9 \mathrm{~Hz}), 25.1$, $24.4(\mathrm{~d}, J=5.0 \mathrm{~Hz})$. HRMS (ESI) m/z: $[\mathrm{M}+\mathrm{H}]^{+}$Calcd for $\mathrm{C}_{18} \mathrm{H}_{20} \mathrm{FN}_{2}$ 283.1605; Found: 283.1610 .

\section{References}

[1] Pacheco, M. C.; Gouverneur, V. Electrophilic Fluorodesilylation of Allenylmethylsilanes: A Novel Entry to 2Fluoro-1,3-dienes. Org. Lett. 2005, 7, 1267-1270.

[2] Tian, M.-Q.; Wang, C.; Hu, X.-H.; Loh, T.-P. Divergent C-H Oxidative Radical Functionalization of Olefins to Install Tertiary Alkyl Motifs Enabled by Copper Catalysis. Org. Lett. 2019, 21, 1607-1611.

[3] Wang, C.; Liu, R.-H.; Tian, M.-Q.; Hu, X.-H.; Loh, T.-P. Regioselective Copper-Catalyzed Oxidative Coupling of $\alpha$-Alkylated Styrenes with Tertiary Alkyl Radicals. Org. Lett. 2018, 20, 4032-4035.

[4] Ono, N.; Tamura, R.; Tanikaga, R.; Kaji, A. Selective Monoalkylation of Tosylacetonitrile. Synthesis 1977, 10, 690-691.

[5] Ghorai, M. K.; Talukdar, R.; Tiwari, D. P. A Route to Highly Functionalized $\beta$-Enaminoesters via a Domino RingOpening Cyclization/Decarboxylative Tautomerization Sequence of Donor-Acceptor Cyclopropanes with Substituted Malononitriles. Org. Lett. 2014, 16, 2204-2207.

[6] Hu, C.; Hong, G.; Qian, X.; Kim, K. R.; Zhu, X.; Wang, L. AlCl3 catalyzed coupling of N-benzylic sulfonamides with 2-substituted cyanoacetates through carbon-nitrogen bond cleavage. Org. Biomol. Chem. 2017, 15, 49844971.

[7] Arceo, E.; Montroni, E.; Melchiorre, P. Photo-Organocatalysis of Atom-Transfer Radical Additions to Alkenes. Angew. Chem., Int. Ed. 2014, 53, 12064-12068.

[8] Ma, Y.; Ding, Y.; Cai, Y.; Lei, C.; Zhu, D.; Li, T. Synthesis and neuroprotective effect of nNOS PDZ structural domain inhibitors. Acta Univ. Med. Nanjing (Natural Science) 2014, 34, 314-322.

[9] Tian, M.-Q.; Shen, Z.-Y.; Zhao, X.; Walsh, P. J.; Hu, X.-H. Iron-Catalyzed Tertiary Alkylation of Terminal Alkynes with 1,3-Diesters via a Functionalized Alkyl Radical. Angew. Chem., Int. Ed. 2021, 60, 9706-9711. 
10. NMR spectra of the starting materials

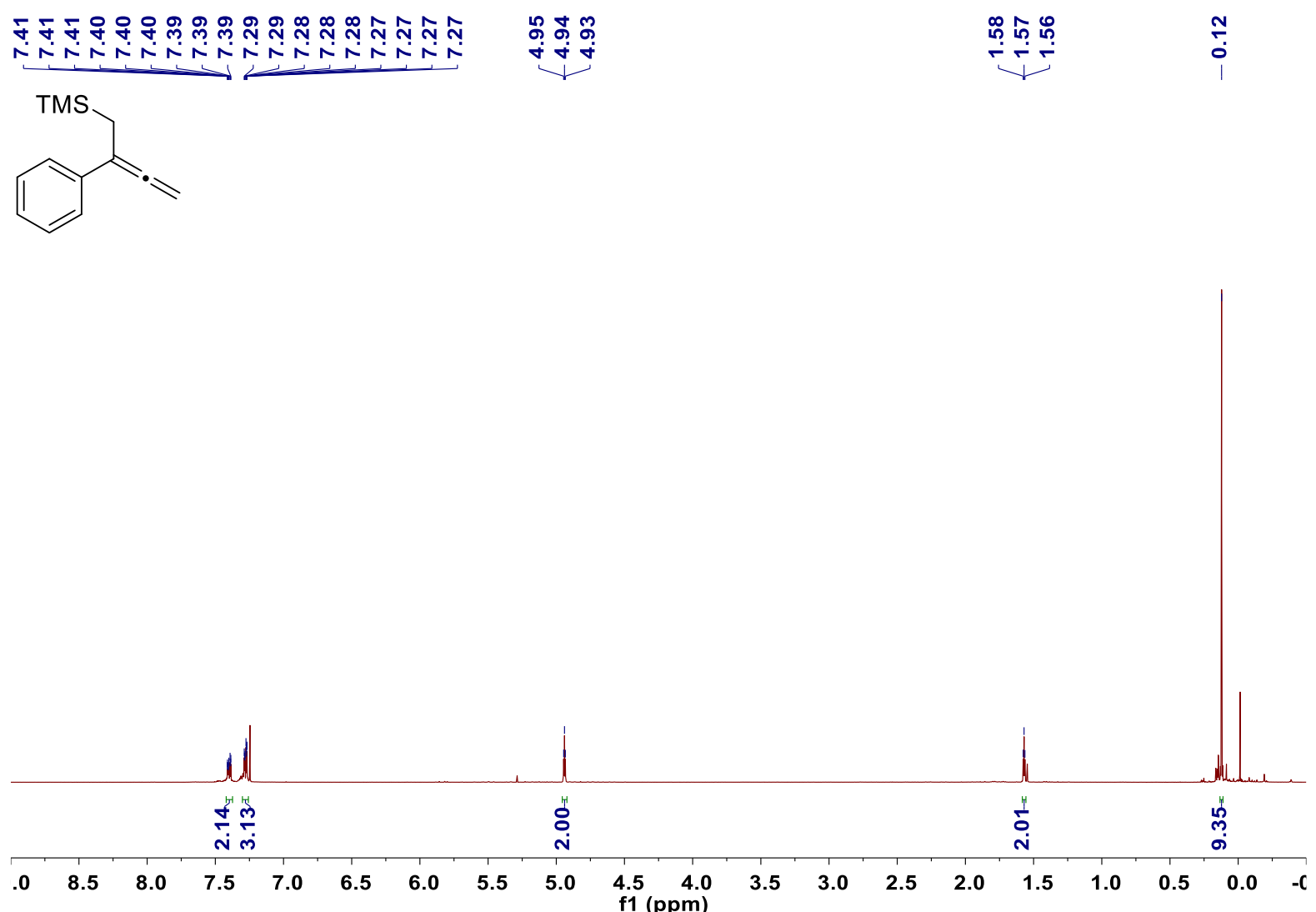

${ }^{1} \mathrm{H}$ NMR spectrum for compound $1 \mathrm{a}\left(400 \mathrm{MHz}, \mathrm{CDCl}_{3}\right)$

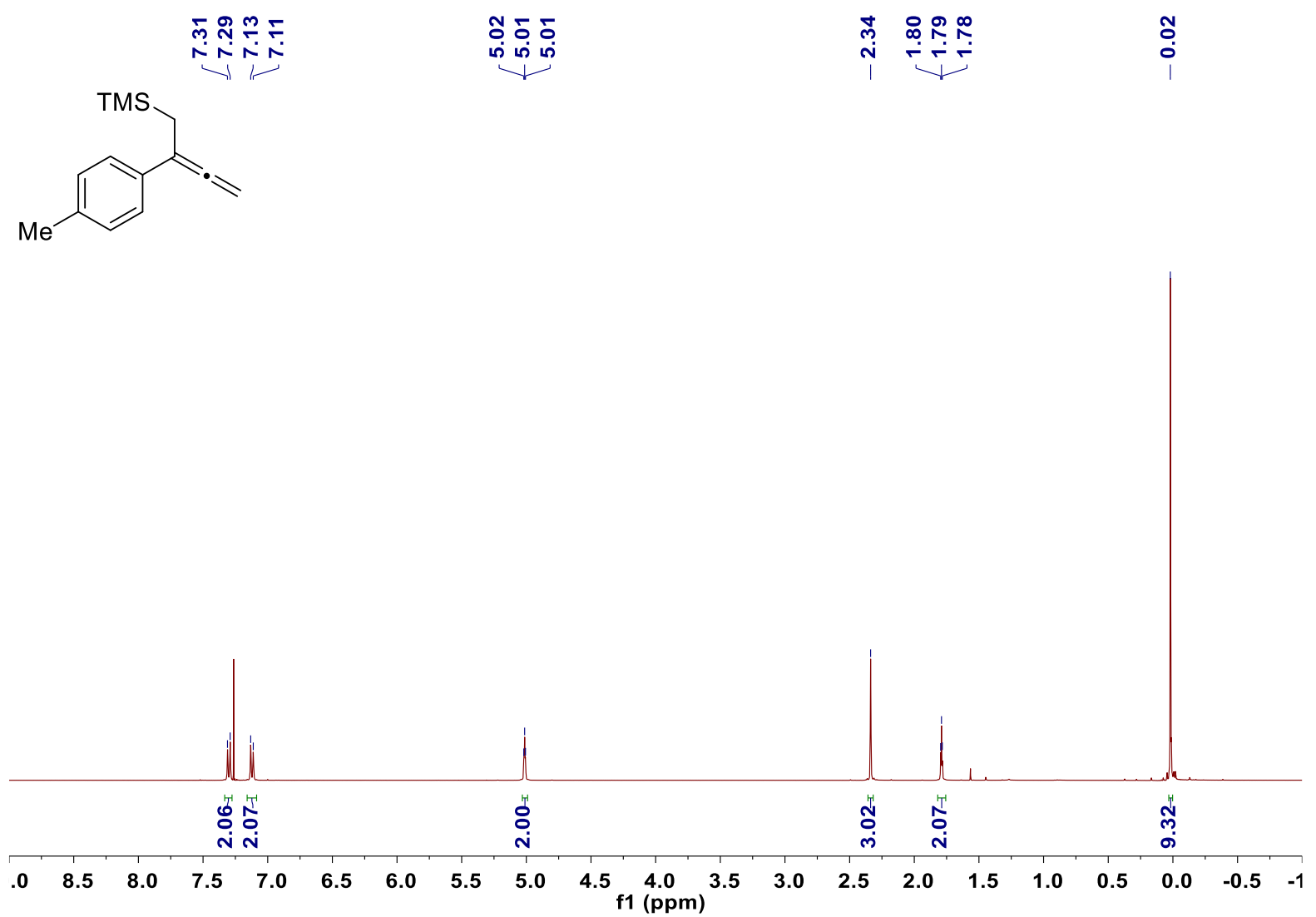




定

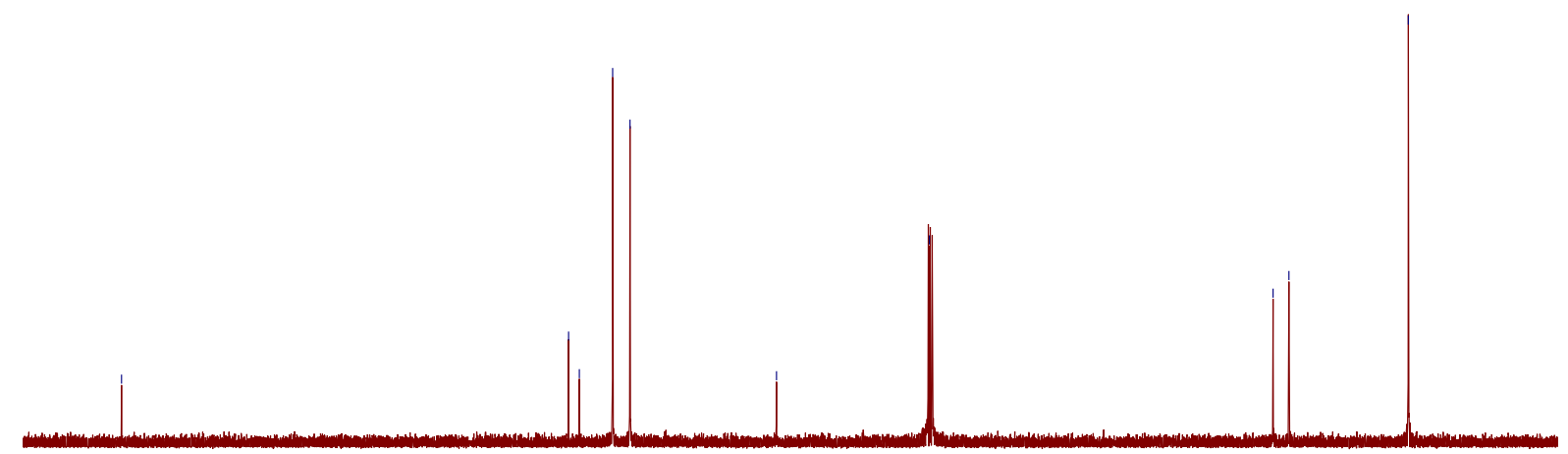

$\begin{array}{lllllllllllllllllllllllllll}220 & 210 & 200 & 190 & 180 & 170 & 160 & 150 & 140 & 130 & 120 & 110 & 100 & 90 & 80 & 70 & 60 & 50 & 40 & 30 & 20 & 10 & 0 & -10 & -20\end{array}$ f1 (ppm)

${ }^{1} \mathrm{H}$ and ${ }^{13} \mathrm{C}$ NMR spectra for compound $1 \mathrm{~b}\left(400 \mathrm{MHz}, \mathrm{CDCl}_{3}\right)$

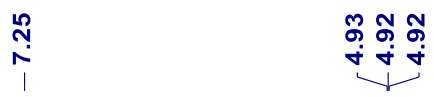

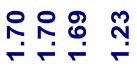

$\stackrel{\circ}{\circ}$<smiles>C=C=C(CS(C)(=O)=O)c1ccc(C(C)(C)C)cc1</smiles>

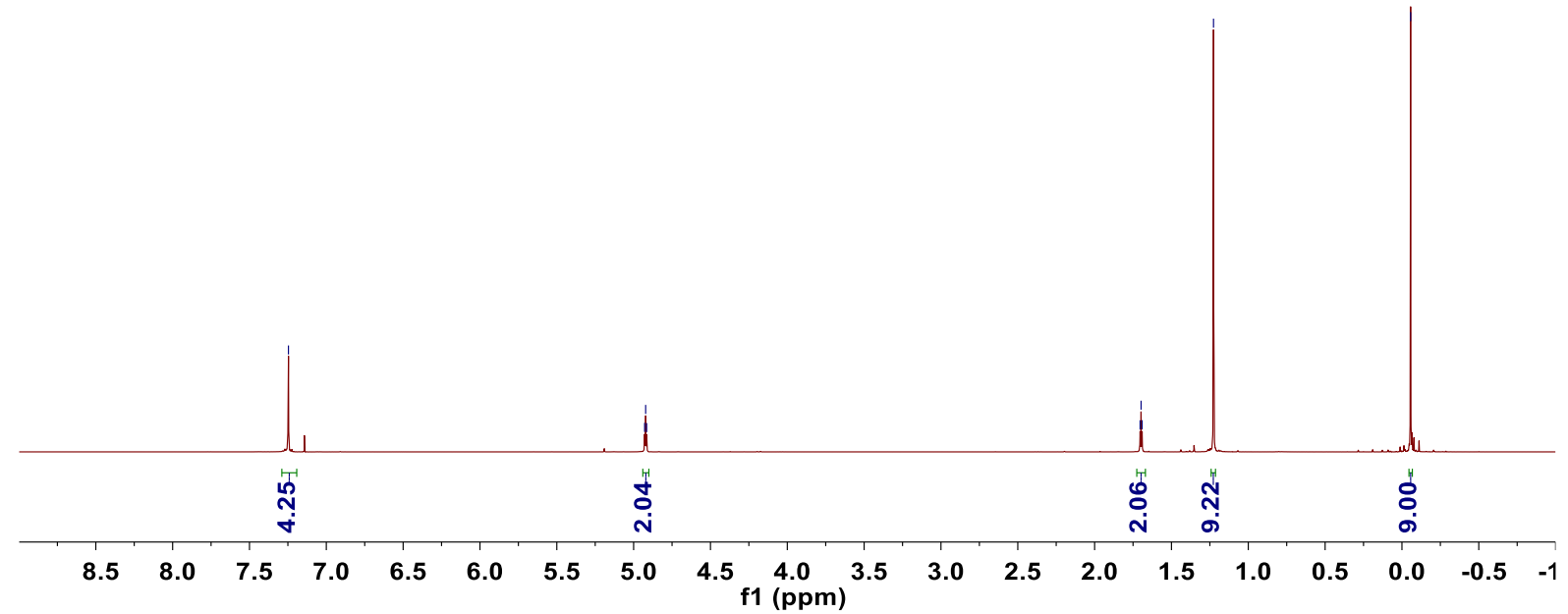




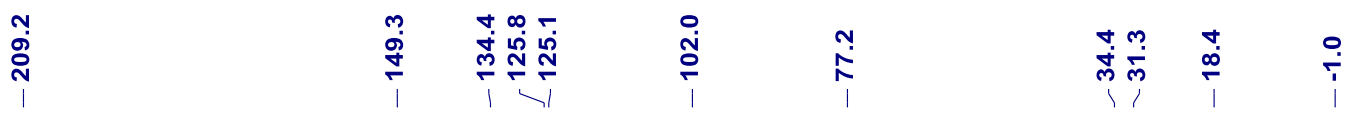

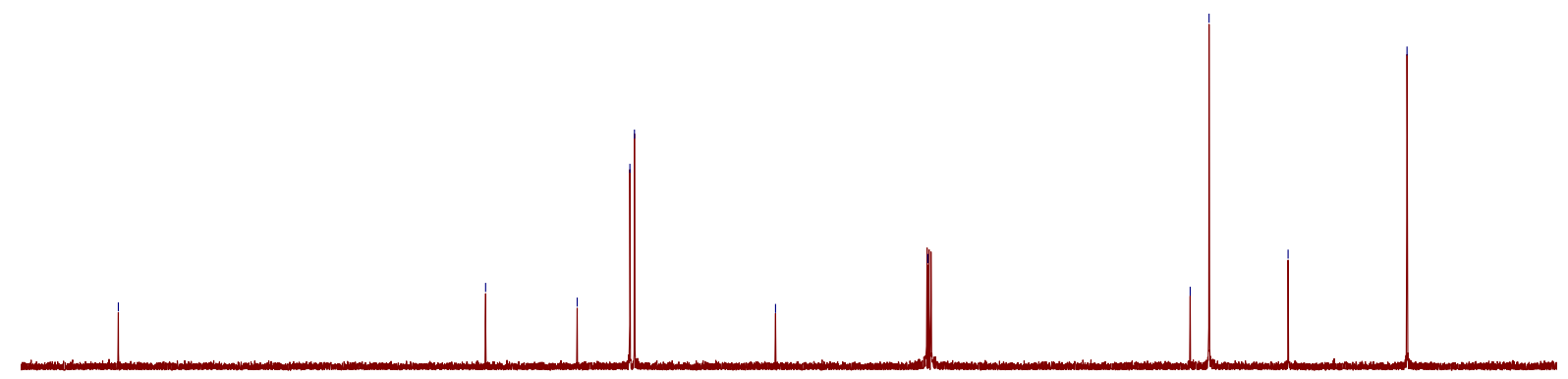

$\begin{array}{llllllllllllllllllllllllllllllll}220 & 210 & 200 & 190 & 180 & 170 & 160 & 150 & 140 & 130 & 120 & 110 & 100 & 90 & 80 & 70 & 60 & 50 & 40 & 30 & 20 & 10 & 0 & -10 & -20\end{array}$ f1 (ppm)

${ }^{1} \mathrm{H}$ and ${ }^{13} \mathrm{C} \mathrm{NMR}$ spectra for compound $1 \mathrm{c}\left(400 \mathrm{MHz}, \mathrm{CDCl}_{3}\right)$

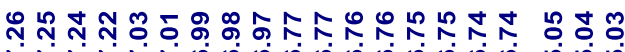

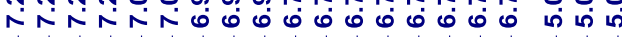

离

¿̊.<smiles>C=C=C(CC)c1cccc(OC)c1</smiles>

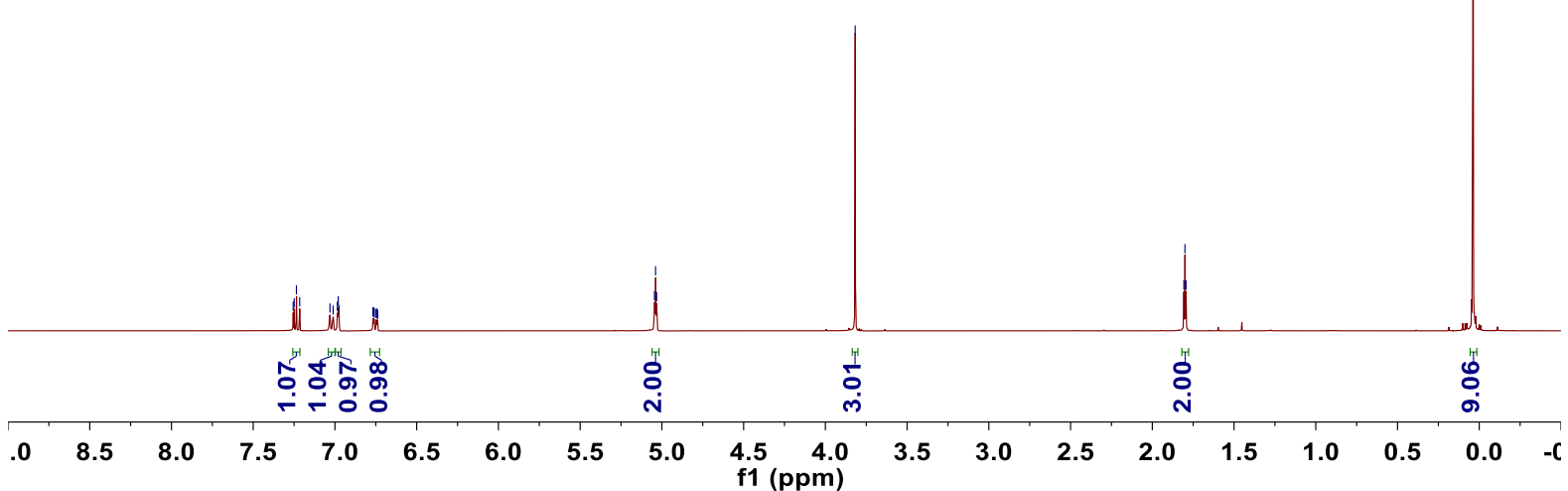




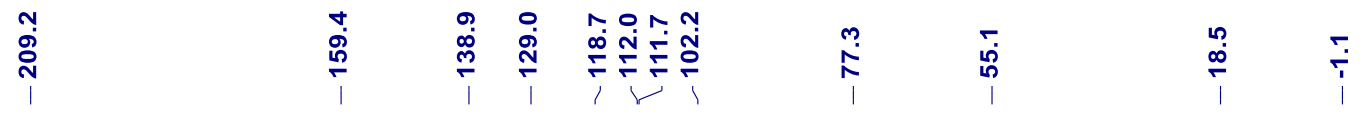

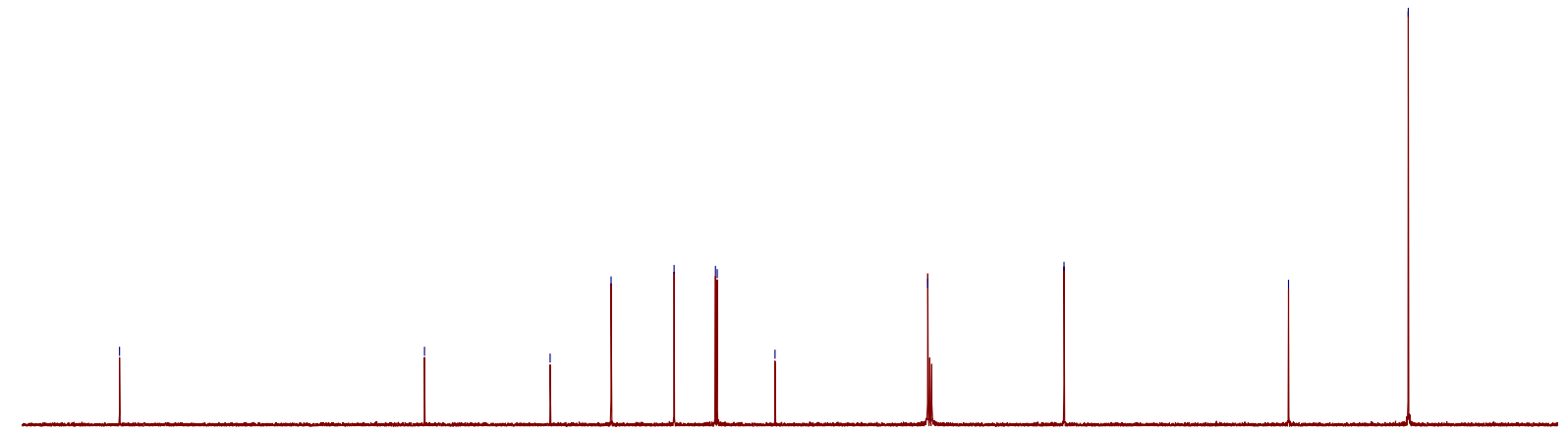
$\begin{array}{llllllllllllllllllllllll}220 & 210 & 200 & 190 & 180 & 170 & 160 & 150 & 140 & 130 & 120 & \begin{array}{c}110 \\ \mathrm{f} 1\end{array}\left(\begin{array}{ll}100 \\ (\mathrm{ppm})\end{array}\right. & \mathbf{9 0} & \mathbf{8 0} & 70 & 60 & 50 & 40 & 30 & 20 & 10 & 0 & -10 & -20\end{array}$

${ }^{1} \mathrm{H}$ and ${ }^{13} \mathrm{C}$ NMR spectra for compound $1 \mathrm{~d}\left(400 \mathrm{MHz}, \mathrm{CDCl}_{3}\right)$

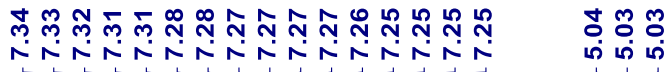

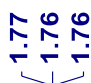

$\grave{0}$<smiles>C=C=C(CS(C)(=O)=O)c1ccc(Cl)cc1</smiles>

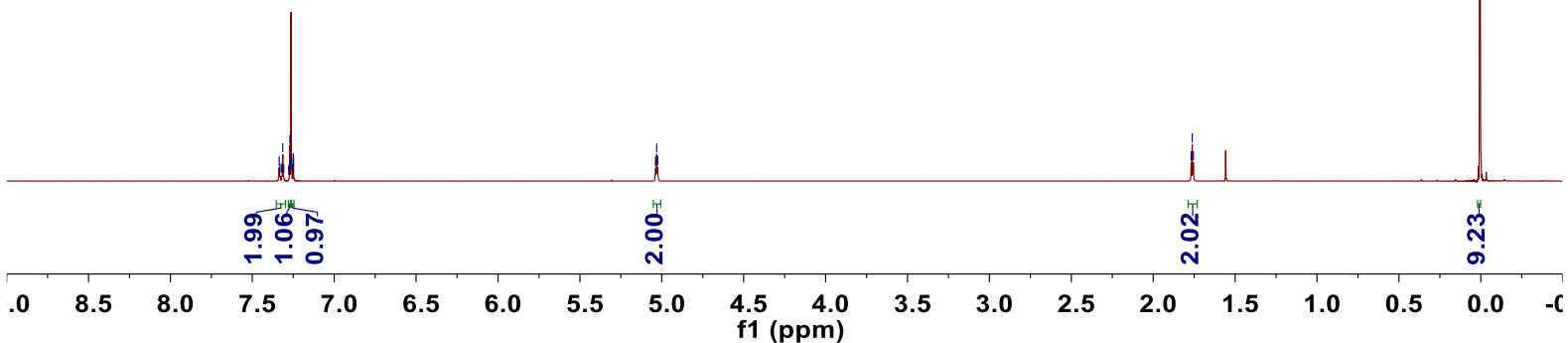




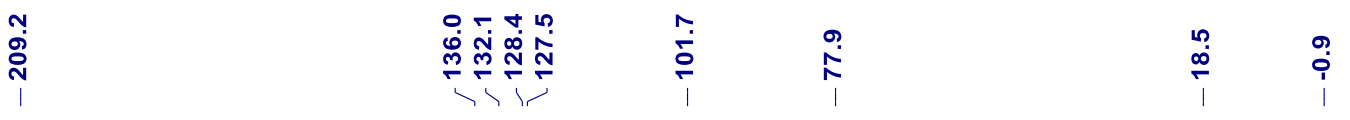

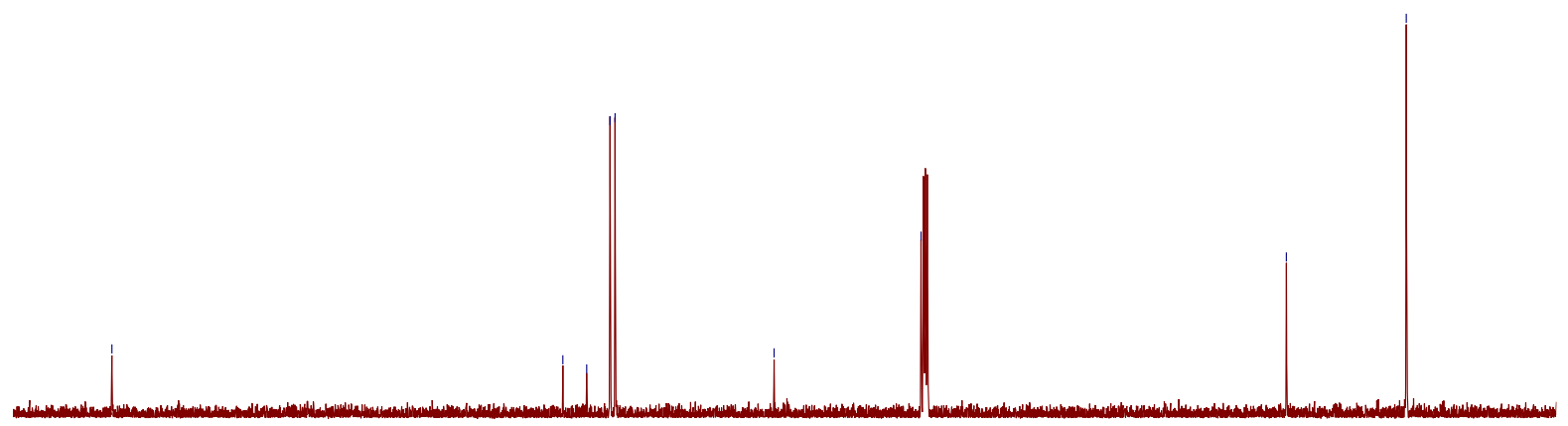

$\begin{array}{llllllllllllllllllllllllllll}220 & 210 & 200 & 190 & 180 & 170 & 160 & 150 & 140 & 130 & 120 & 110 & 100 & 90 & 80 & 70 & 60 & 50 & 40 & 30 & 20 & 10 & 0 & -10 & -20\end{array}$ f1 (ppm)

${ }^{1} \mathrm{H}$ and ${ }^{13} \mathrm{C}$ NMR spectra for compound $1 \mathrm{e}\left(400 \mathrm{MHz}, \mathrm{CDCl}_{3}\right)$

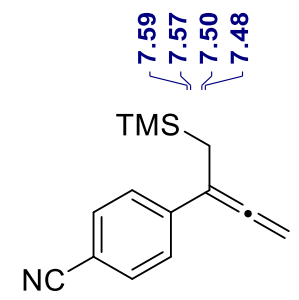

琯

ํㅜㄹํํㄹ

พั

$\mathrm{NC}$

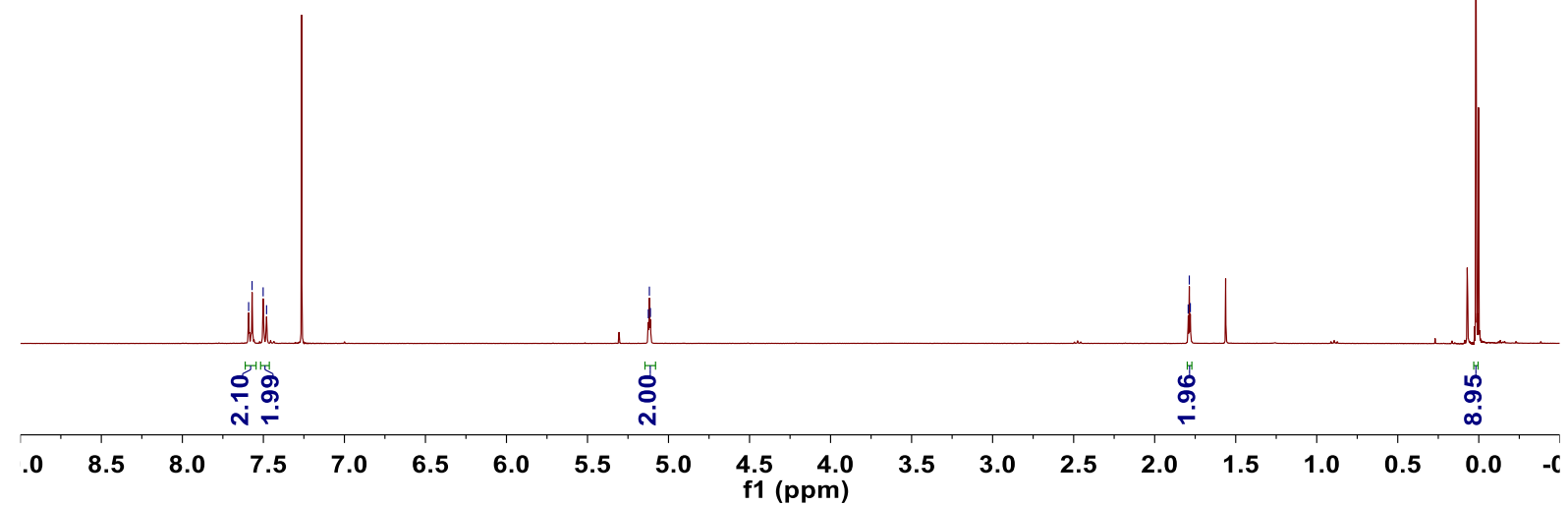




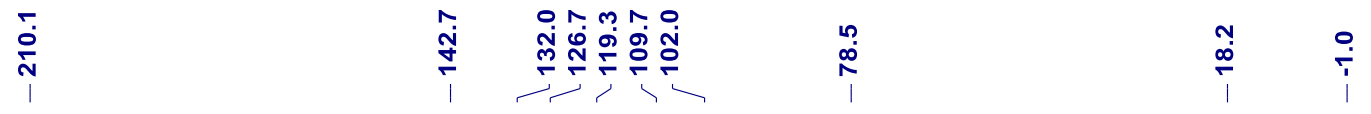

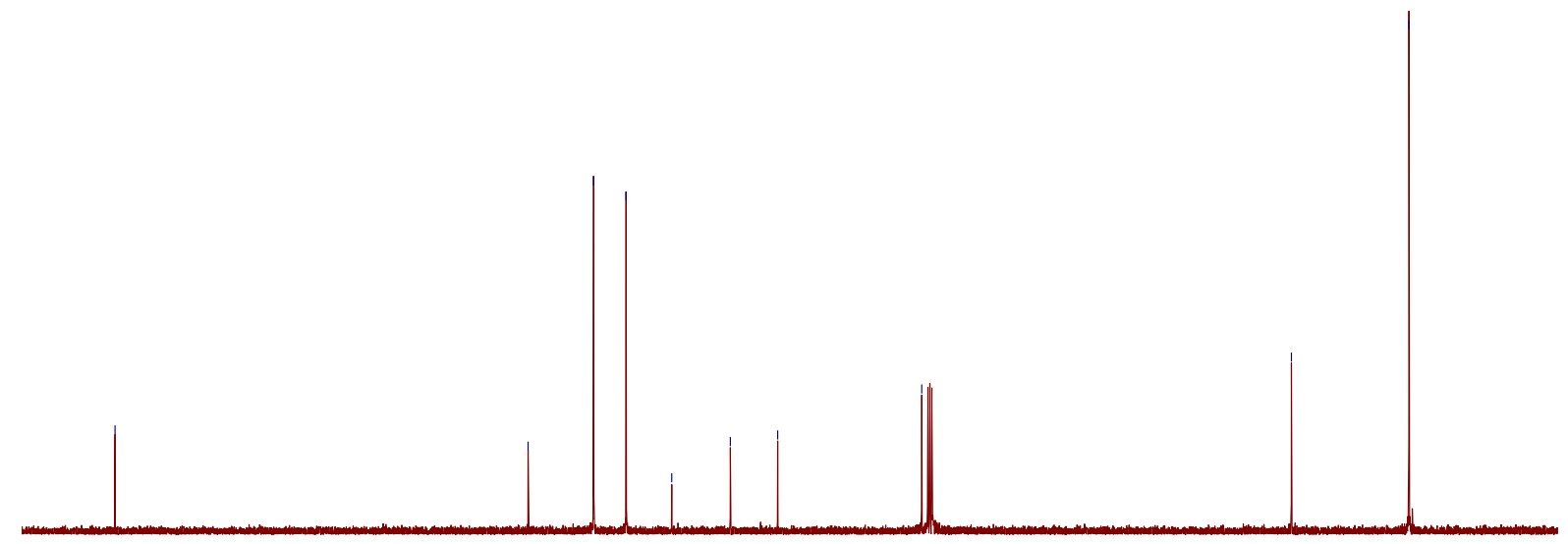

$\begin{array}{lllllllllllllllllllllllll}220 & 210 & 200 & 190 & 180 & 170 & 160 & 150 & 140 & 130 & 120 & 110 & 100 & 90 & 80 & 70 & 60 & 50 & 40 & 30 & 20 & 10 & 0 & -10 & -20\end{array}$ f1 (ppm)

${ }^{1} \mathrm{H}$ and ${ }^{13} \mathrm{C}$ NMR spectra for compound $1 \mathrm{f}\left(400 \mathrm{MHz}, \mathrm{CDCl}_{3}\right)$<smiles>C=C=C(CS)c1ccc(C(F)(F)F)cc1</smiles>

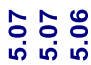

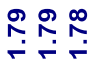

:

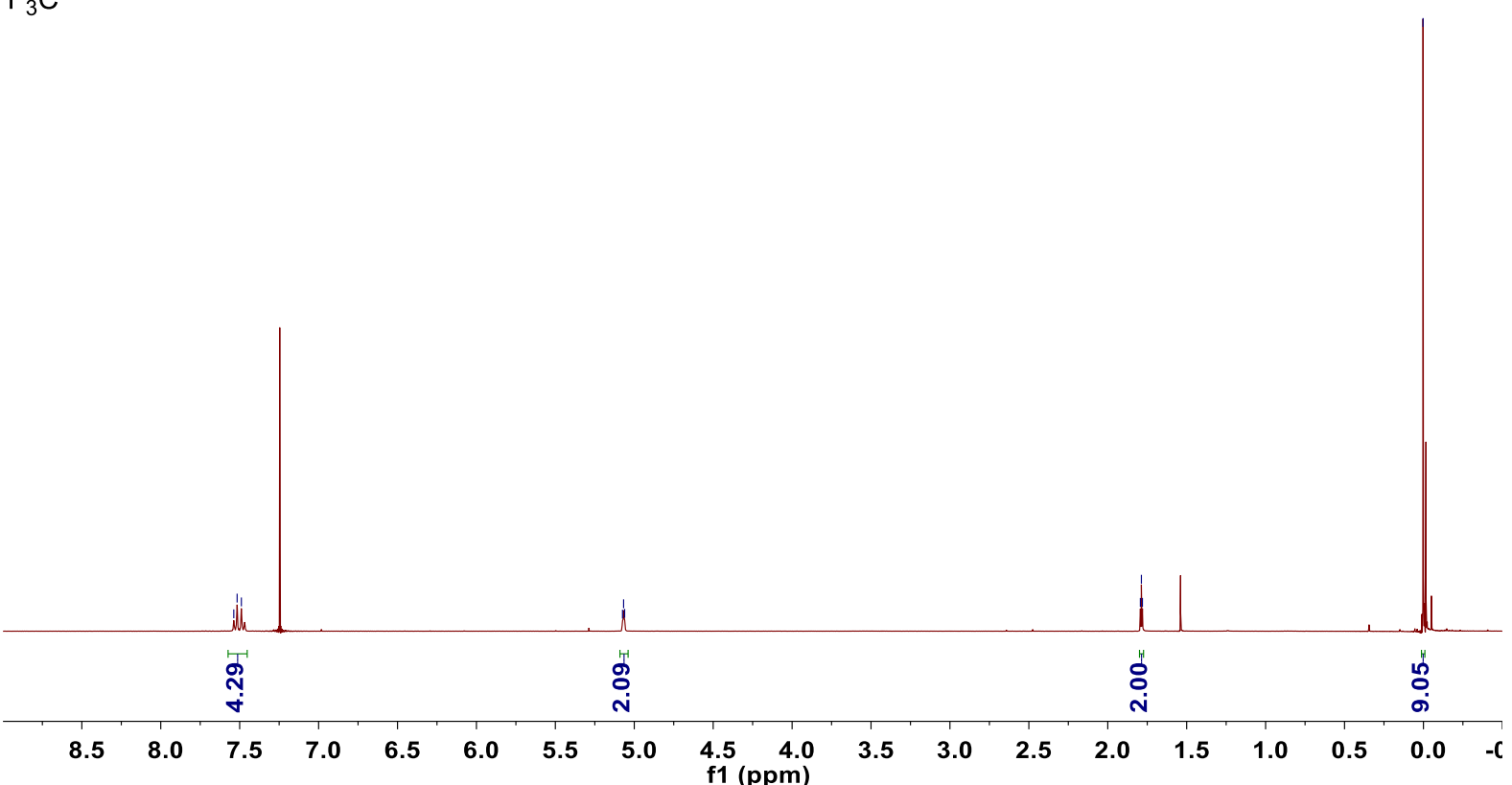




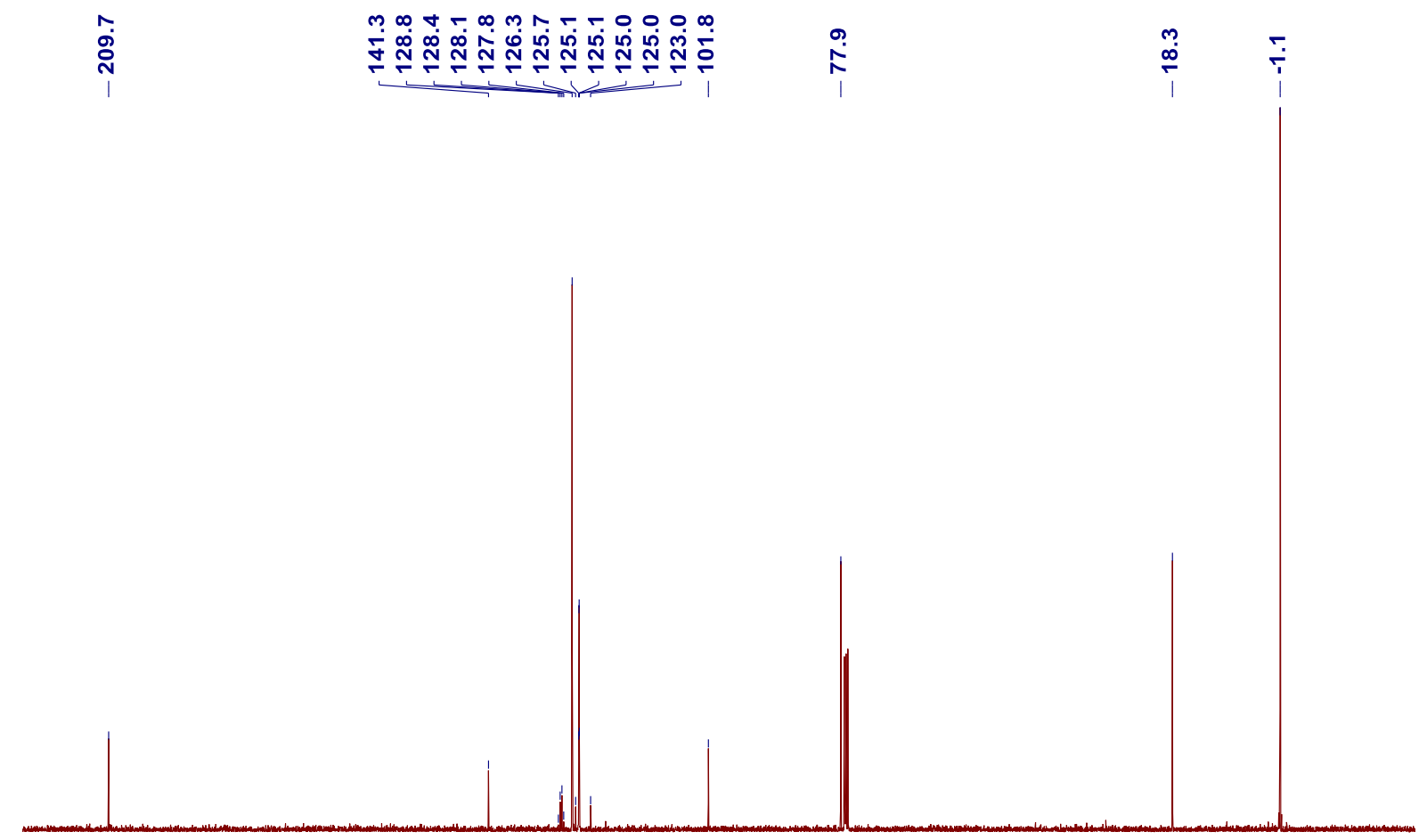

$\begin{array}{llllllllllllllllllllllllllll}220 & 210 & 200 & 190 & 180 & 170 & 160 & 150 & 140 & 130 & 120 & 110 & 100 & 90 & 80 & 70 & 60 & 50 & 40 & 30 & 20 & 10 & 0 & -10 & -20\end{array}$ f1 (ppm)

ஸุ.

$\begin{array}{llllllllllllllllllllll}100 & 80 & 60 & 40 & 20 & 0 & -20 & -40 & -60 & -80 & -100 & -120 & -140 & -160 & -180 & -200 & -220 & -240 & -260 & -280 & -30 C\end{array}$ f1 (ppm)

${ }^{1} \mathrm{H},{ }^{13} \mathrm{C}$ and ${ }^{19} \mathrm{~F}$ NMR spectra for compound $1 \mathrm{~g}\left(400 \mathrm{MHz}, \mathrm{CDCl}_{3}\right)$ 


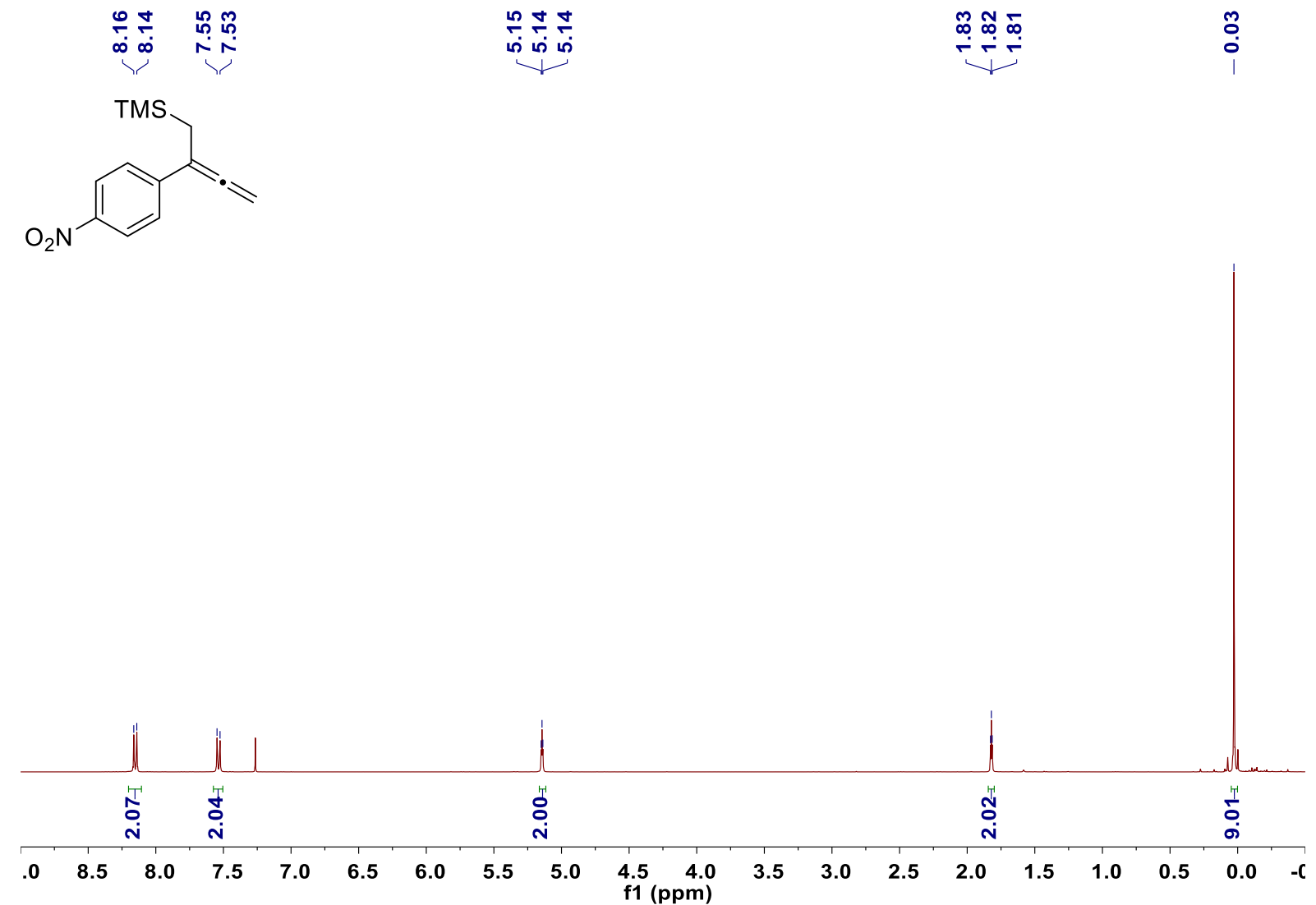

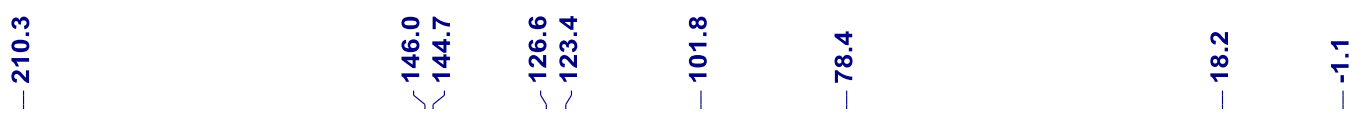

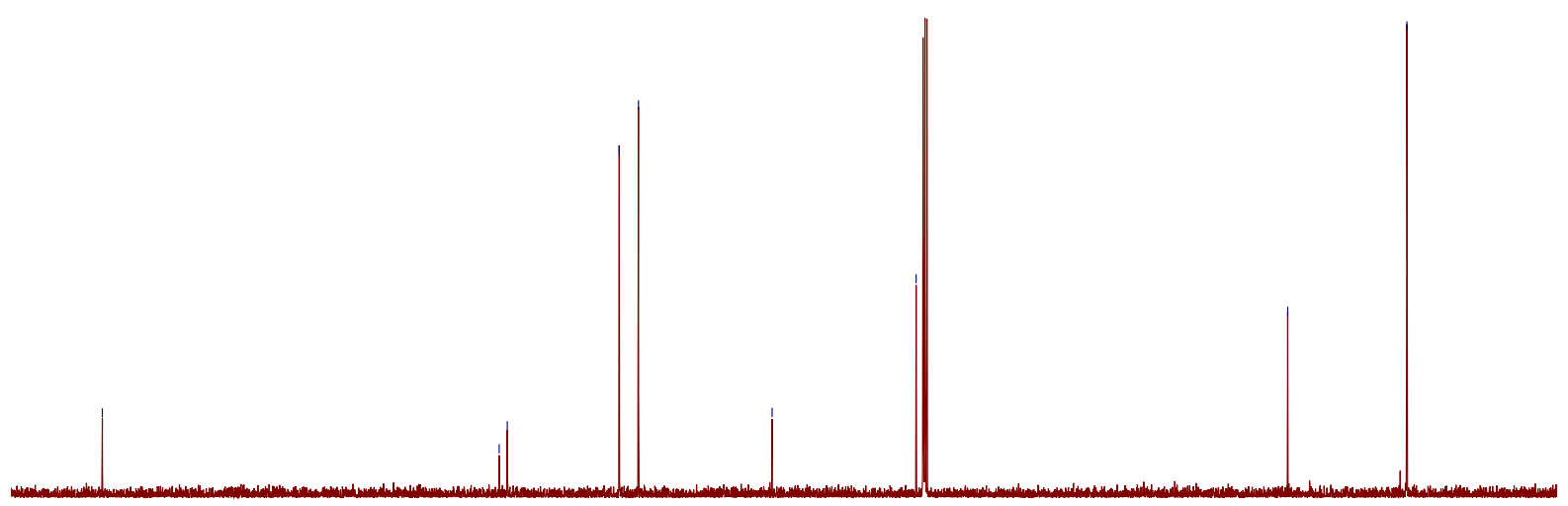

$\begin{array}{llllllllllllllllllllllllllll}220 & 210 & 200 & 190 & 180 & 170 & 160 & 150 & 140 & 130 & 120 & 110 & 100 & 90 & 80 & 70 & 60 & 50 & 40 & 30 & 20 & 10 & 0 & -10 & -20\end{array}$ f1 (ppm)

${ }^{1} \mathrm{H}$ and ${ }^{13} \mathrm{C} \mathrm{NMR}$ spectra for compound $1 \mathrm{~h}\left(400 \mathrm{MHz}, \mathrm{CDCl}_{3}\right)$ 


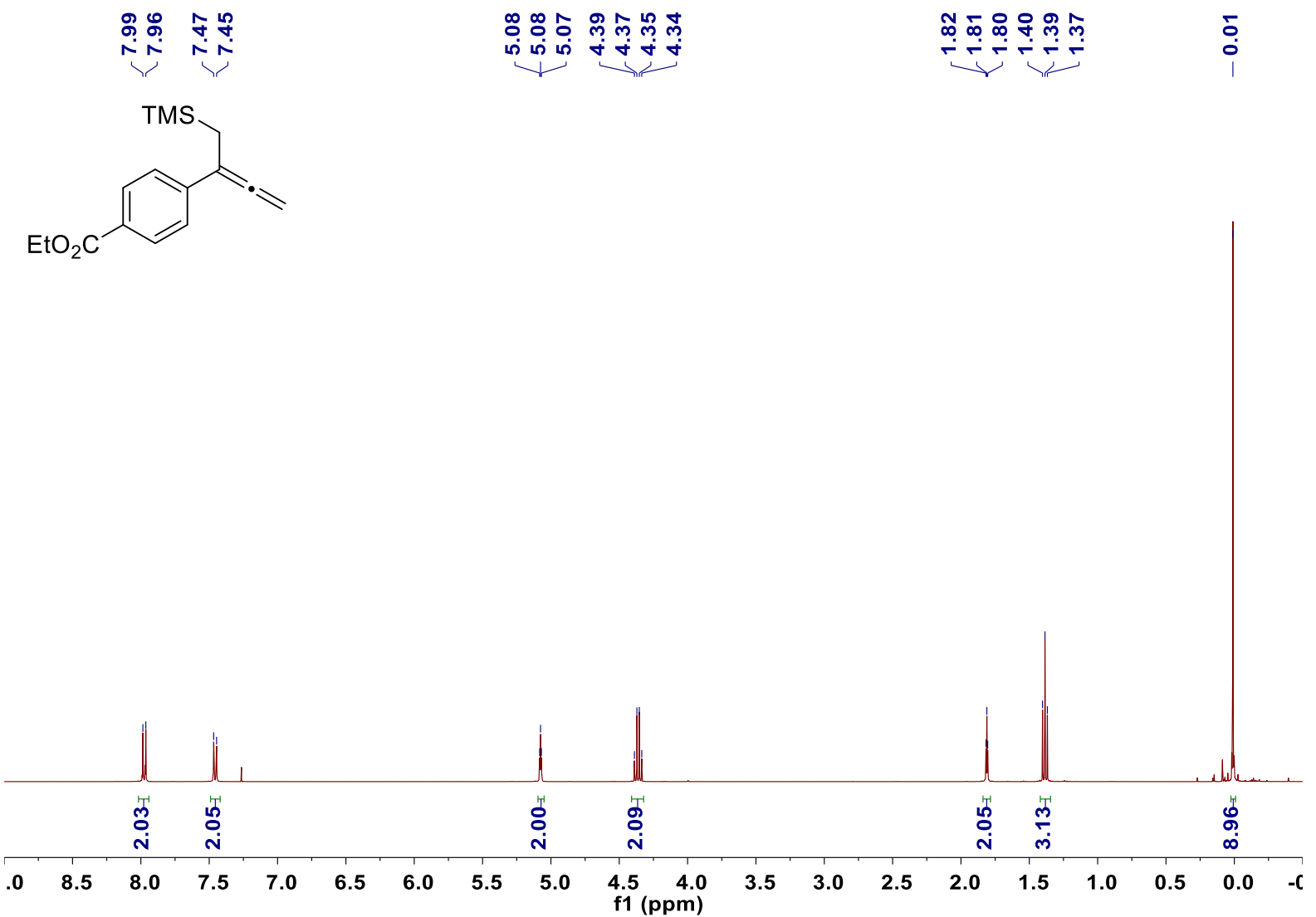

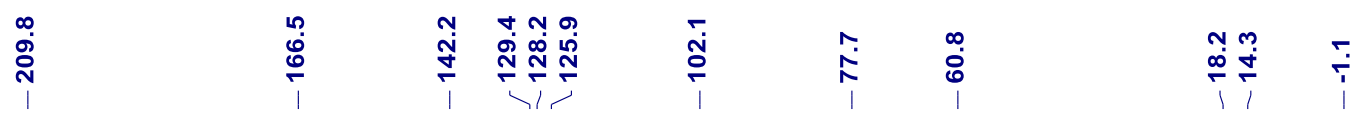

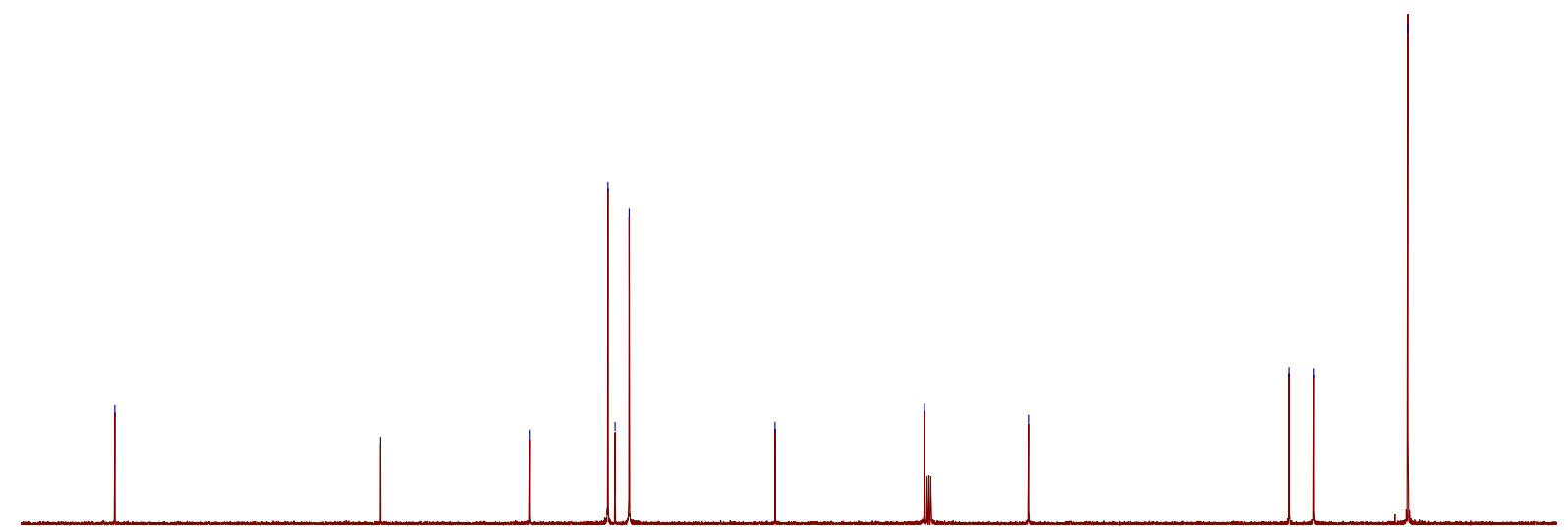

$\begin{array}{llllllllllllllllllllllllllllllll}220 & 210 & 200 & 190 & 180 & 170 & 160 & 150 & 140 & 130 & 120 & 110 & 100 & 90 & 80 & 70 & 60 & 50 & 40 & 30 & 20 & 10 & 0 & -10 & -20\end{array}$ f1 (ppm)

${ }^{1} \mathrm{H}$ and ${ }^{13} \mathrm{C}$ NMR spectra for compound $1 \mathrm{i}\left(400 \mathrm{MHz}, \mathrm{CDCl}_{3}\right)$ 


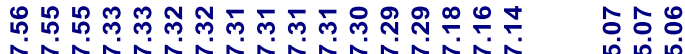

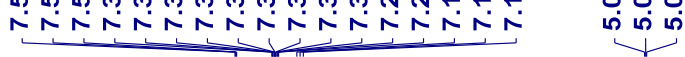

손요

id<smiles>C=C=C(CC)c1cccc(Br)c1</smiles>

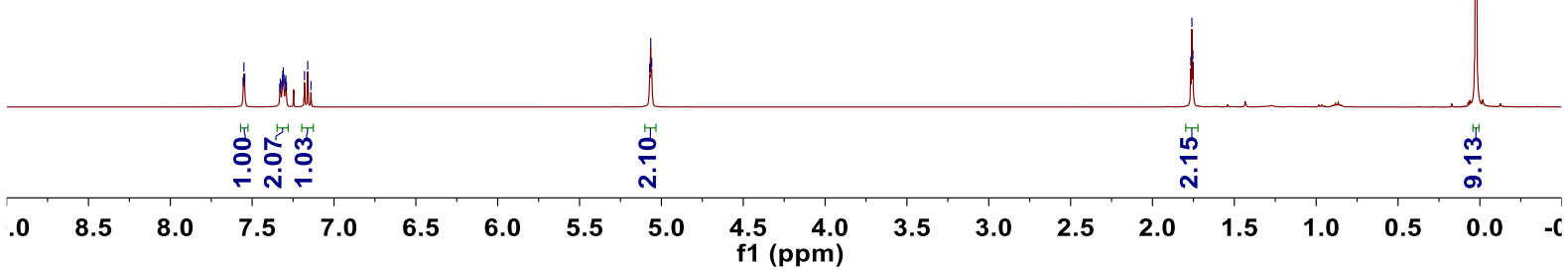

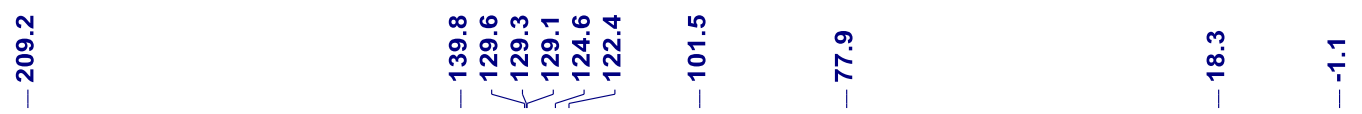

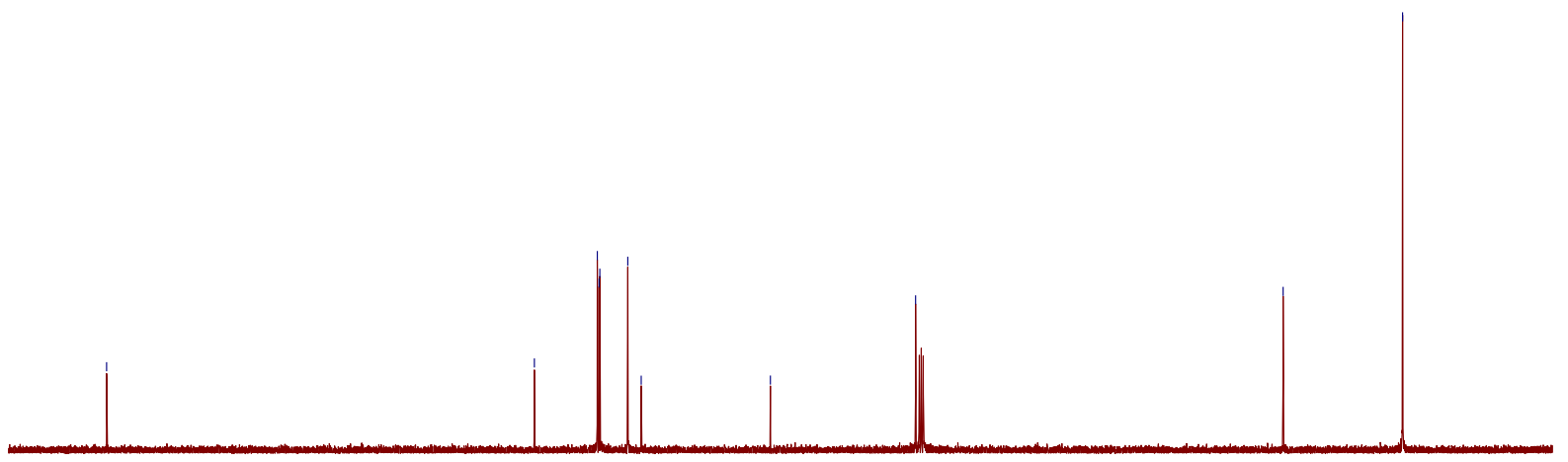

$\begin{array}{lllllllllllllllllllllllll}220 & 210 & 200 & 190 & 180 & 170 & 160 & 150 & 140 & 130 & 120 & 110 & 100 & 90 & 80 & 70 & 60 & 50 & 40 & 30 & 20 & 10 & 0 & -10 & -20\end{array}$ f1 (ppm)

${ }^{1} \mathrm{H}$ and ${ }^{13} \mathrm{C}$ NMR spectra for compound $1 \mathrm{j}\left(400 \mathrm{MHz}, \mathrm{CDCl}_{3}\right)$ 


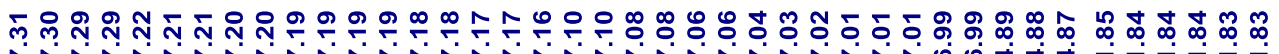

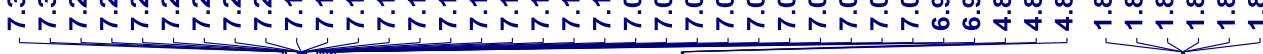<smiles>C=C=C(CS)c1ccccc1F</smiles>

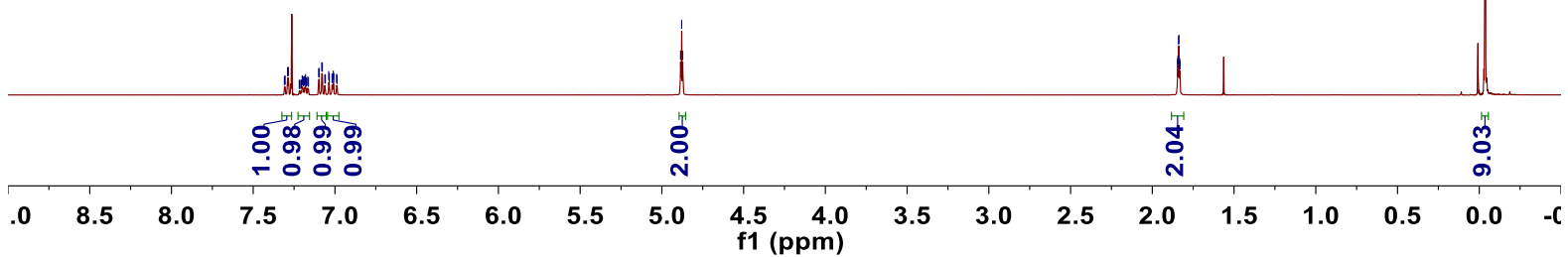

คั่

Tุ

产点

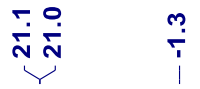

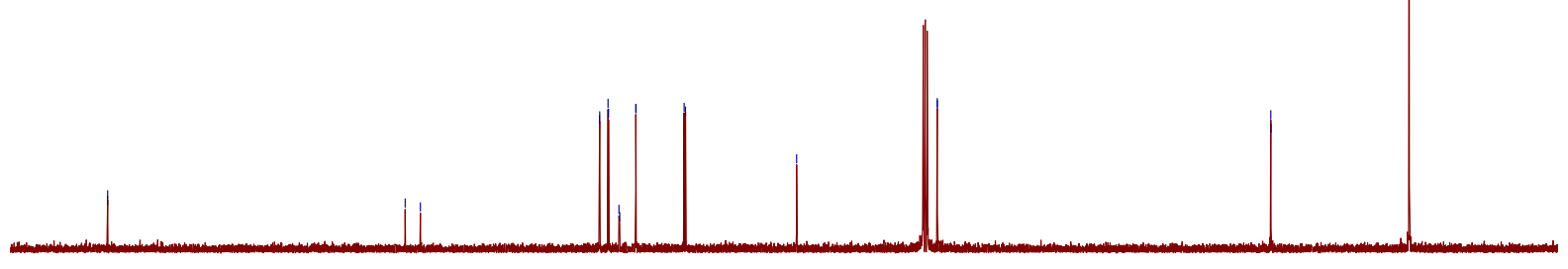

$\begin{array}{llllllllllllllllllllllllllllllll}220 & 210 & 200 & 190 & 180 & 170 & 160 & 150 & 140 & 130 & 120 & 110 & 100 & 90 & 80 & 70 & 60 & 50 & 40 & 30 & 20 & 10 & 0 & -10 & -20\end{array}$ f1 (ppm) 


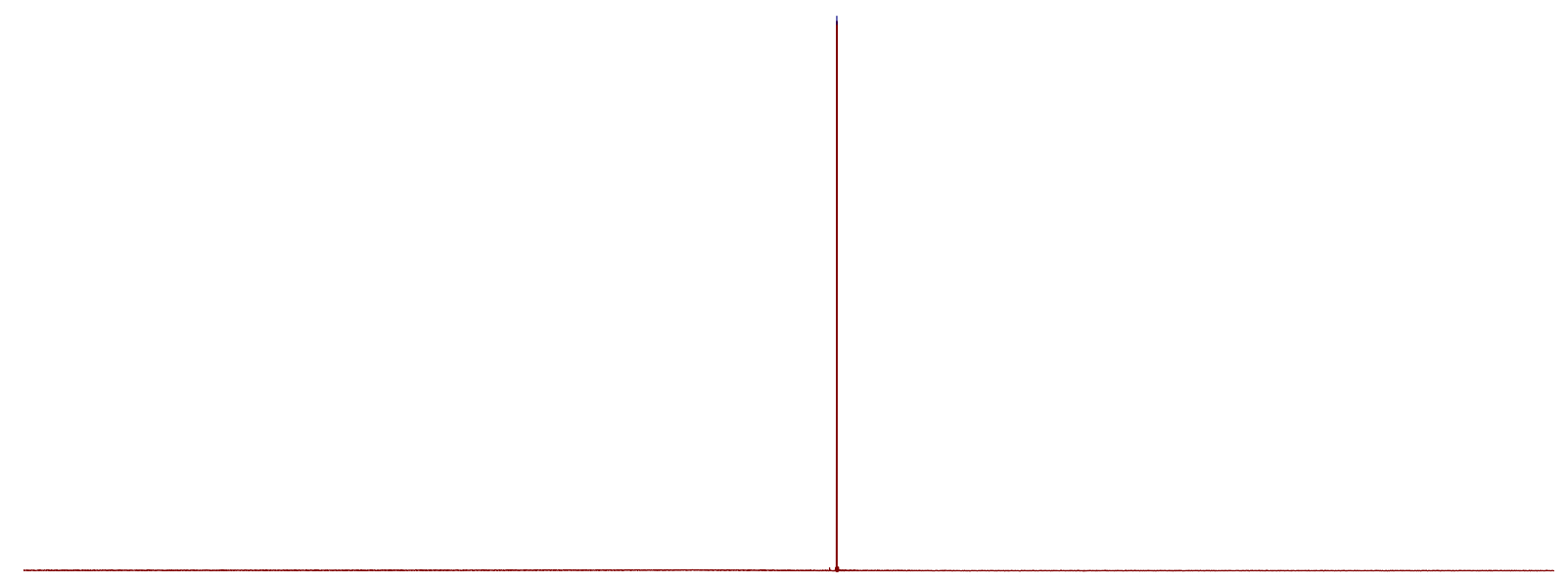

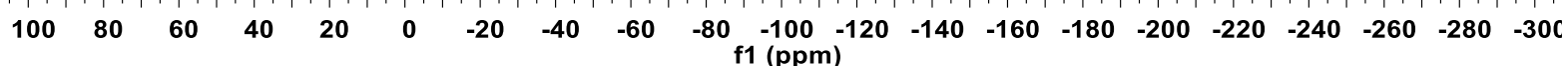

${ }^{1} \mathrm{H},{ }^{13} \mathrm{C}$ and ${ }^{19} \mathrm{~F}$ NMR spectra for compound $1 \mathrm{k}\left(400 \mathrm{MHz}, \mathrm{CDCl}_{3}\right)$

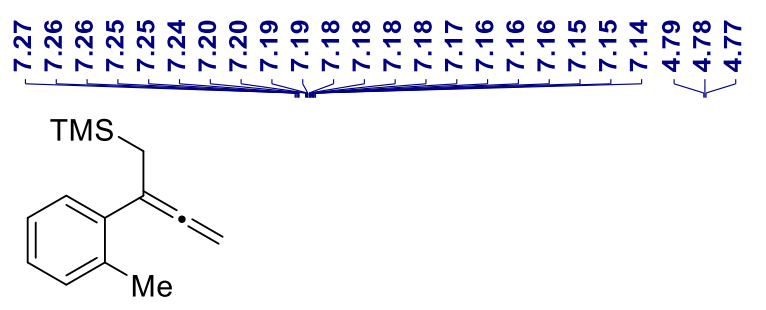

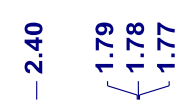

$\stackrel{8}{i}$

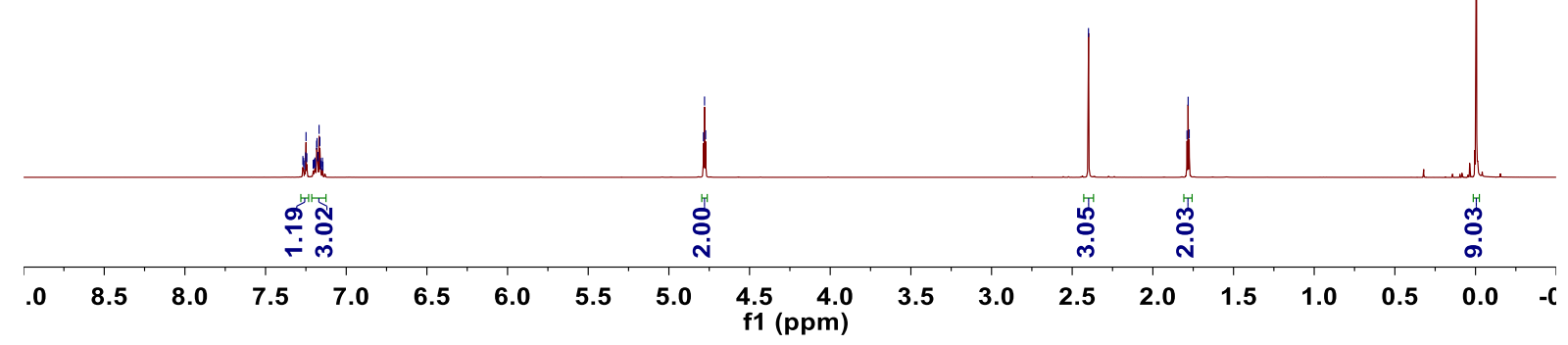


กิ

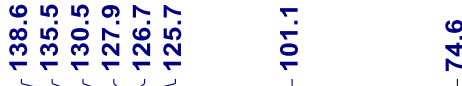

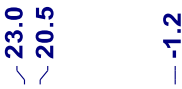

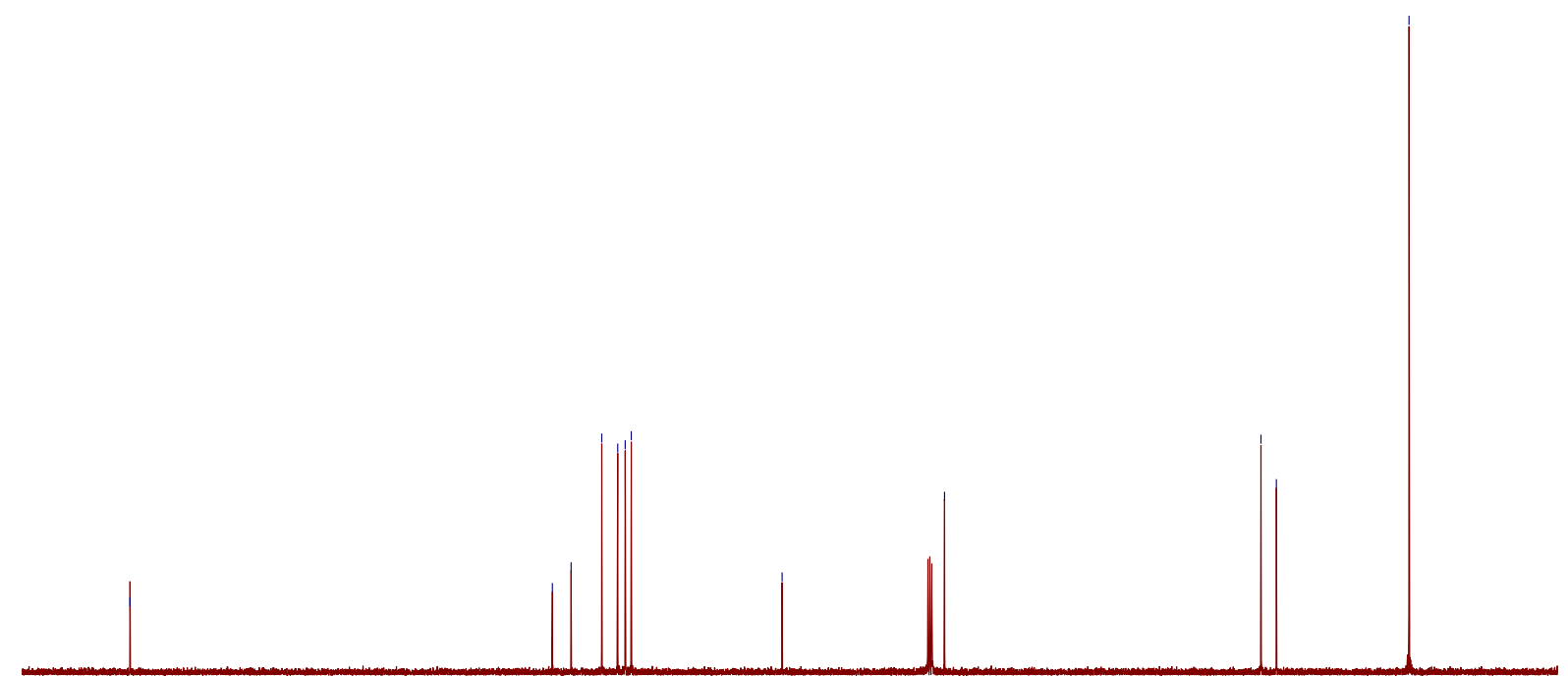

$\begin{array}{lllllllllllllllllllllllllll}220 & 210 & 200 & 190 & 180 & 170 & 160 & 150 & 140 & 130 & 120 & 110 & 100 & 90 & 80 & 70 & 60 & 50 & 40 & 30 & 20 & 10 & 0 & -10 & -20\end{array}$ f1 (ppm)

${ }^{1} \mathrm{H}$ and ${ }^{13} \mathrm{C}$ NMR spectra for compound $1 \mathrm{l}\left(400 \mathrm{MHz}, \mathrm{CDCl}_{3}\right)$

๑ ㅇํㅇำ

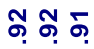

$\stackrel{1}{\circ}$

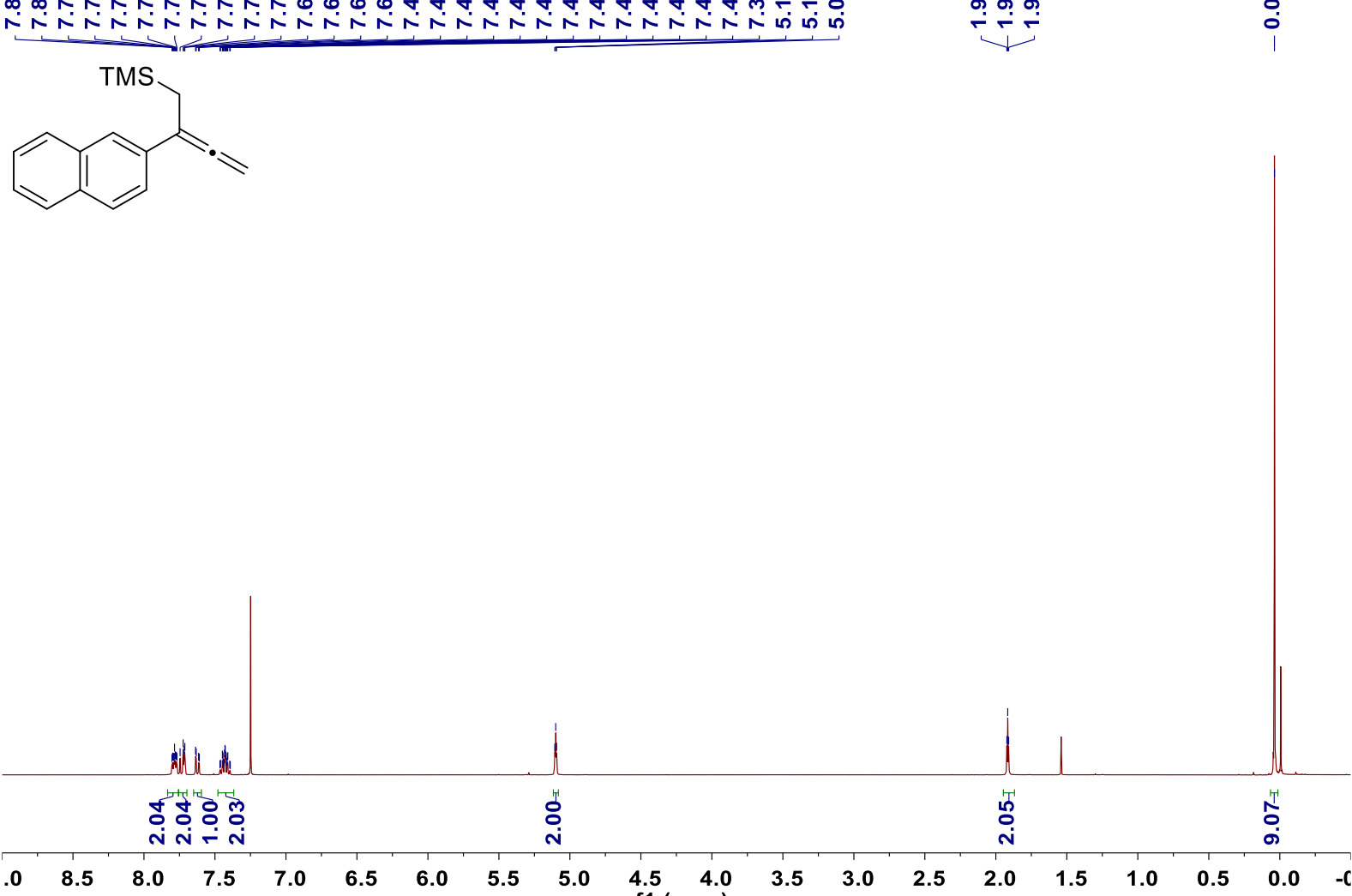
f1 (ppm) 
@̣̊

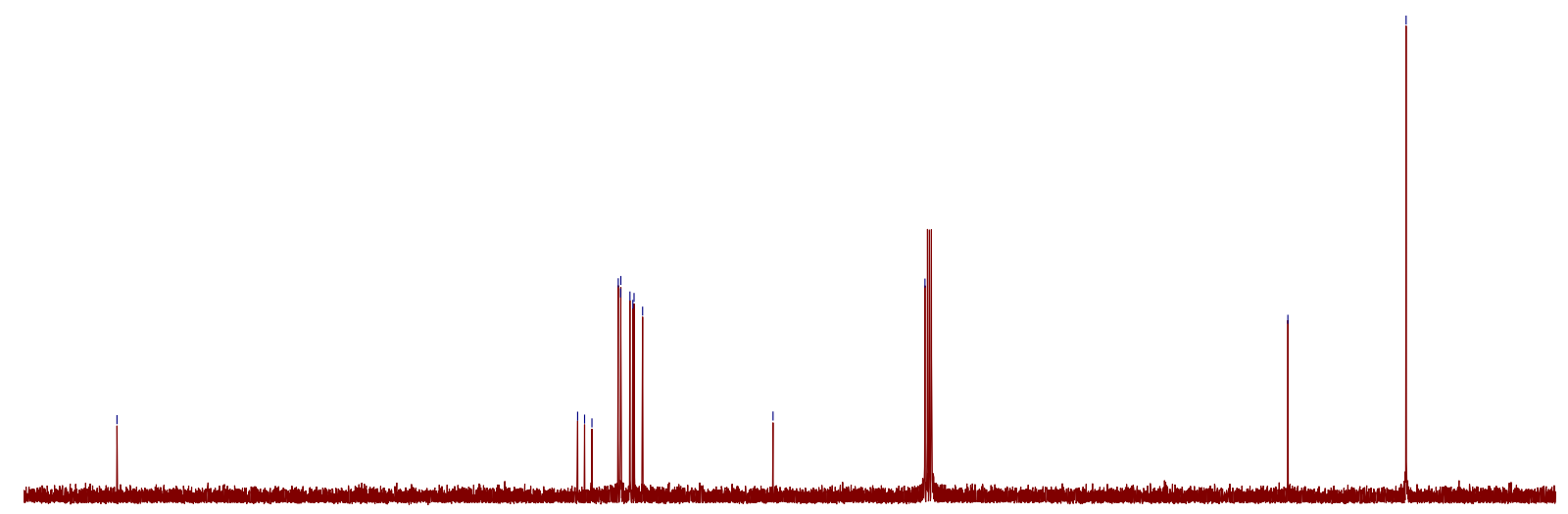

$\begin{array}{lllllllllllll}220 & 210 & 200 & 190 & 180 & 170 & 160 & 150 & 140 & 130 & 120 & 110 & 100 \\ \mathrm{f} 1 & (\mathrm{ppm})\end{array}$

${ }^{1} \mathrm{H}$ and ${ }^{13} \mathrm{C}$ NMR spectra for compound $1 \mathrm{~m}\left(400 \mathrm{MHz}, \mathrm{CDCl}_{3}\right)$

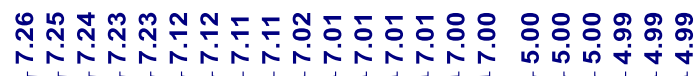

華雚<smiles>C=C=C(CS)c1ccsc1</smiles>

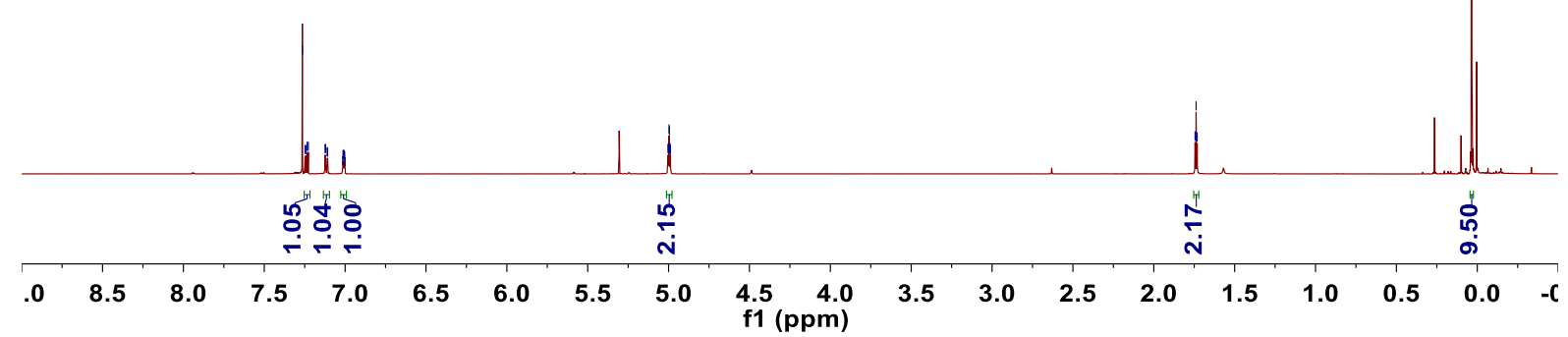




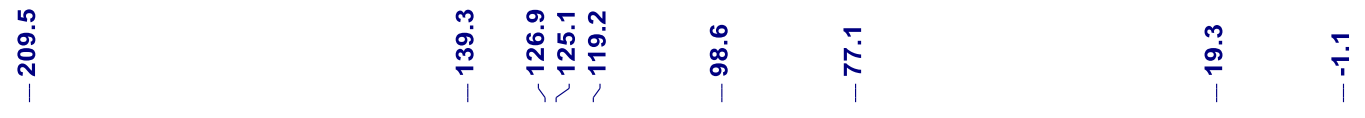

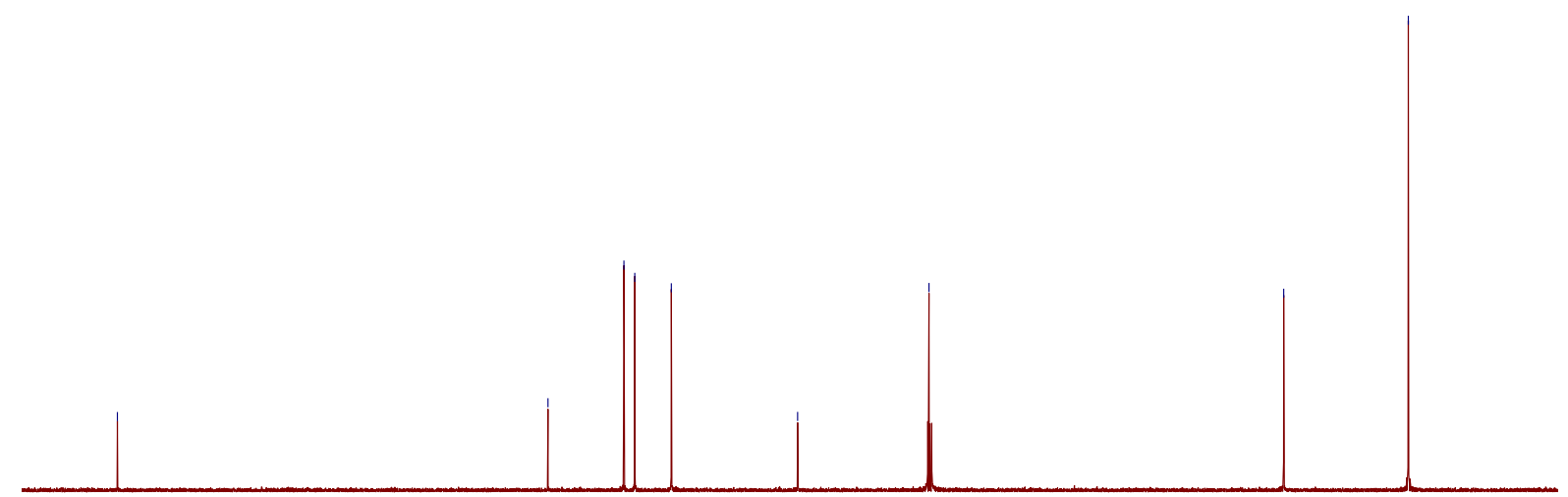

$\begin{array}{lllllllllllllllllllllllll}220 & 210 & 200 & 190 & 180 & 170 & 160 & 150 & 140 & 130 & 120 & 110 & 100 & 90 & 80 & 70 & 60 & 50 & 40 & 30 & 20 & 10 & 0 & -10 & -20\end{array}$ f1 (ppm)

${ }^{1} \mathrm{H}$ and ${ }^{13} \mathrm{C}$ NMR spectra for compound $1 \mathrm{n}\left(400 \mathrm{MHz}, \mathrm{CDCl}_{3}\right)$

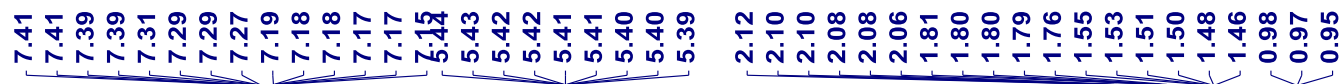
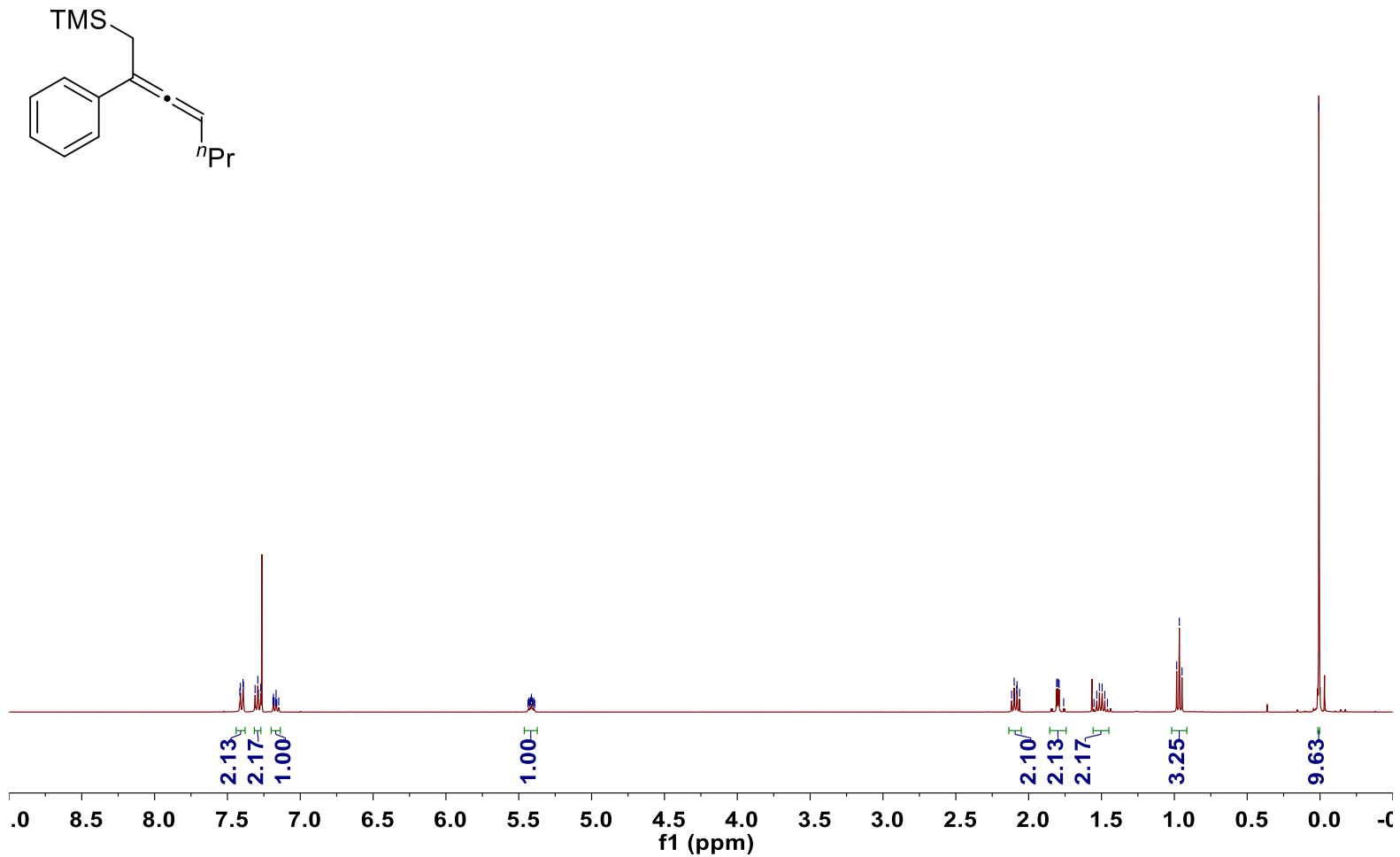
จั่

ํำ

ז்

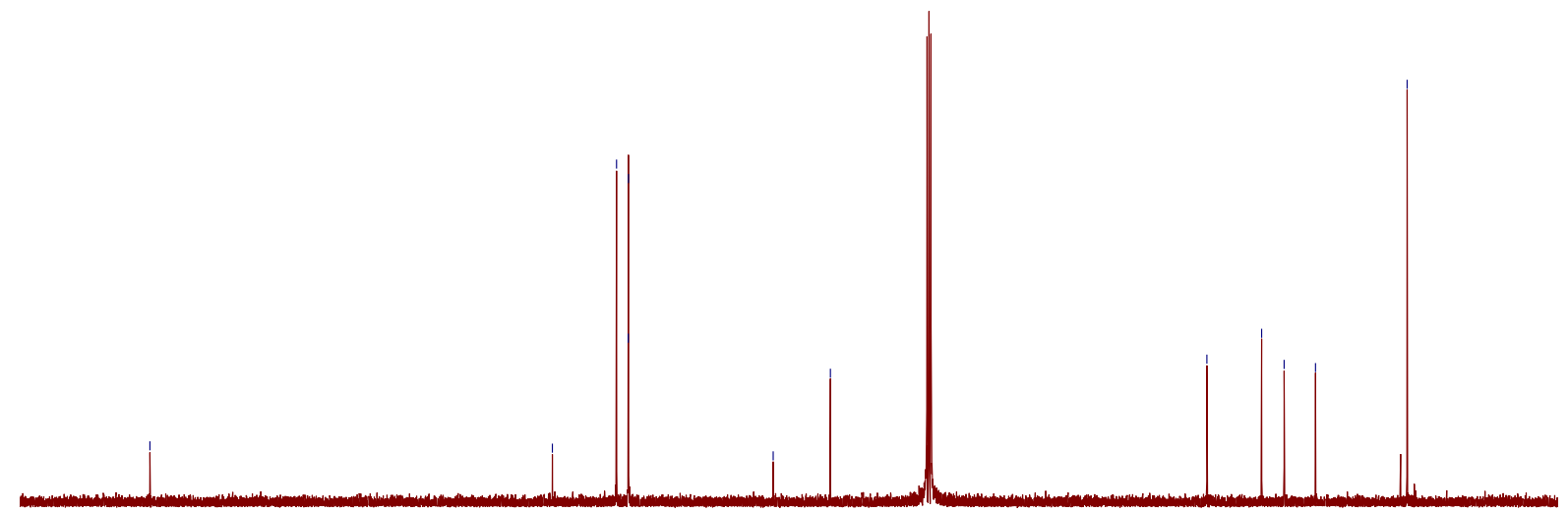

$\begin{array}{lllllllllllllllllllllllll}220 & 210 & 200 & 190 & 180 & 170 & 160 & 150 & 140 & 130 & 120 & 110 & 100 & 90 & 80 & 70 & 60 & 50 & 40 & 30 & 20 & 10 & 0 & -10 & -20\end{array}$ f1 (ppm)

${ }^{1} \mathrm{H}$ and ${ }^{13} \mathrm{C}$ NMR spectra for compound $10\left(400 \mathrm{MHz}, \mathrm{CDCl}_{3}\right)$

テテ요 있

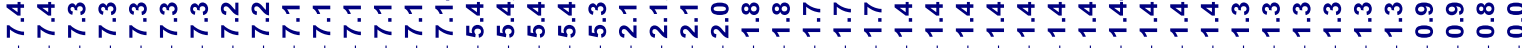<smiles>CCCC=C=C(CS)c1ccccc1</smiles>

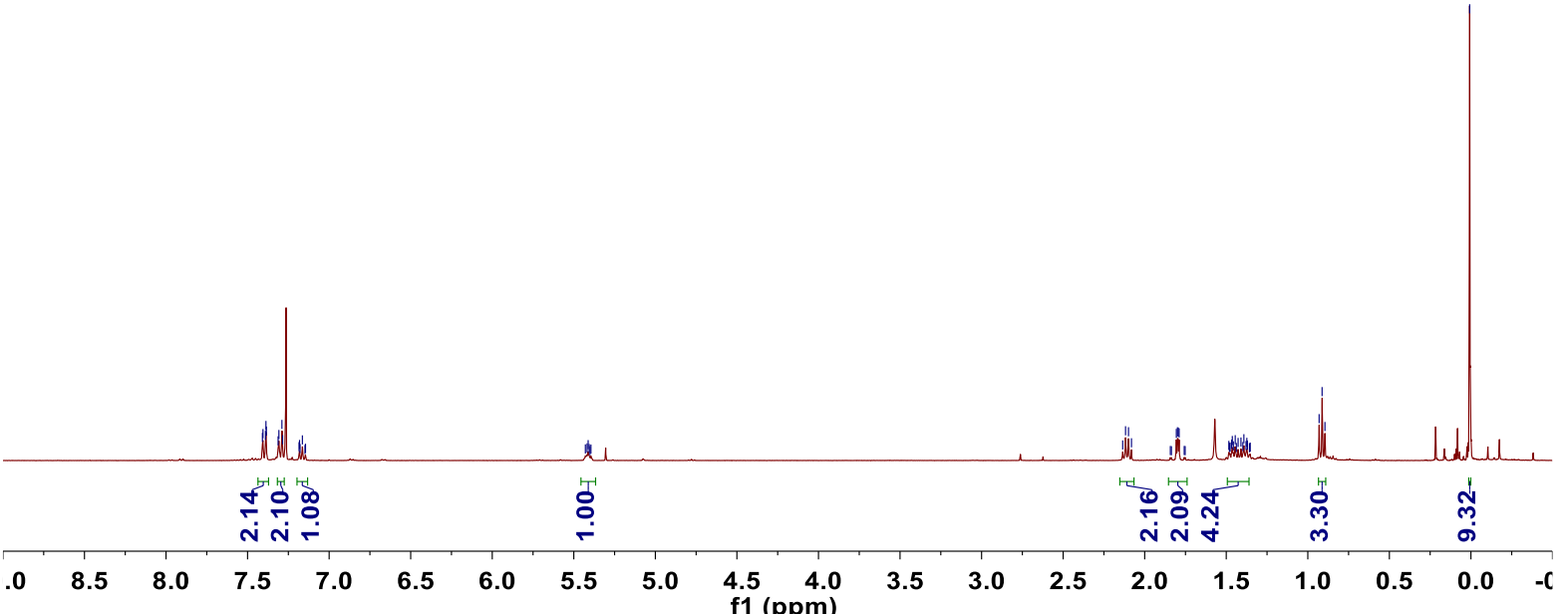


ָั่

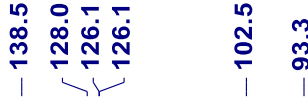

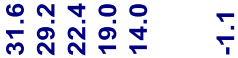

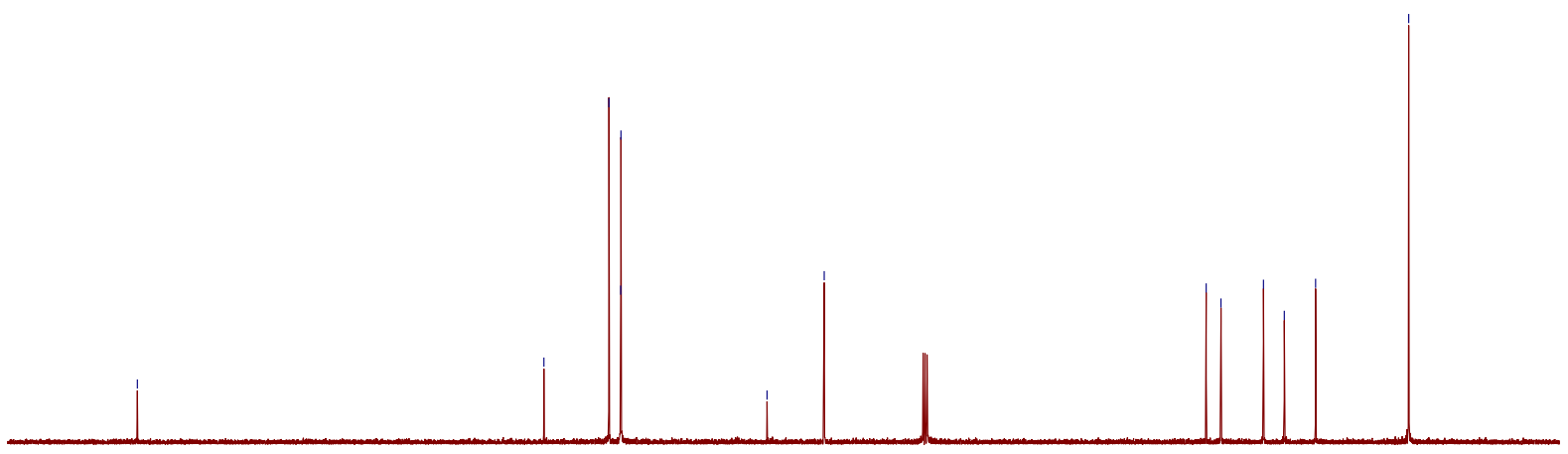

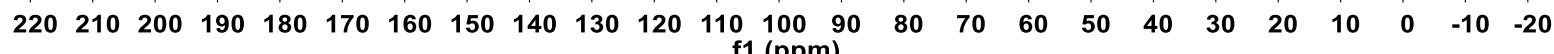

${ }^{1} \mathrm{H}$ and ${ }^{13} \mathrm{C}$ NMR spectra for compound $1 \mathrm{p}\left(400 \mathrm{MHz}, \mathrm{CDCl}_{3}\right)$

ติ<smiles>CSC(=C=CCCc1ccccc1)c1ccccc1</smiles>

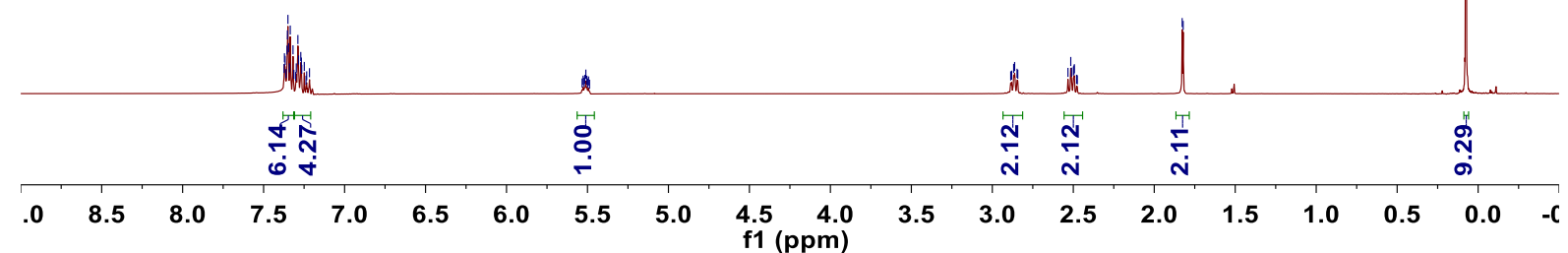


$\stackrel{m}{\stackrel{m}{5}}$

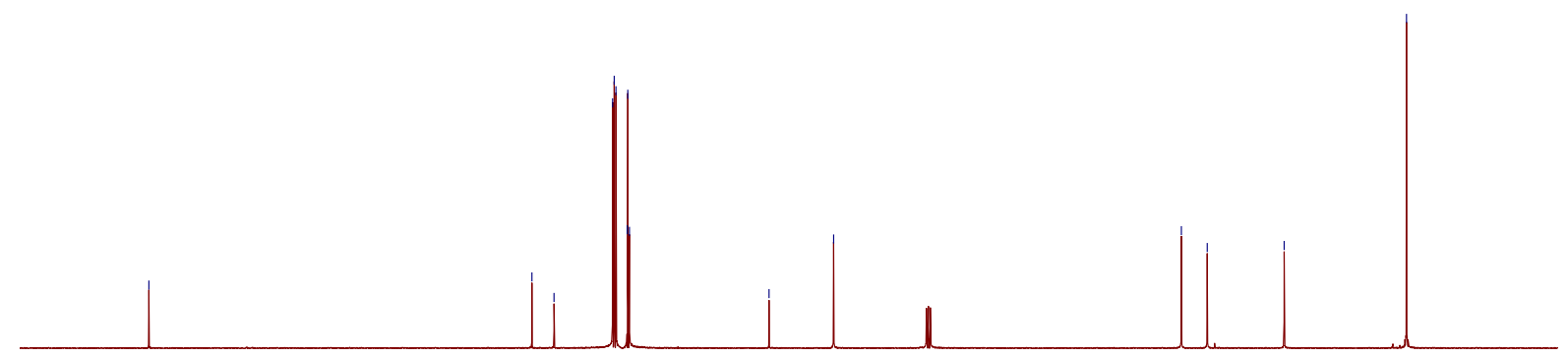

$\begin{array}{lllllllllllllllllllllll}220 & 210 & 200 & 190 & 180 & 170 & 160 & 150 & 140 & 130 & 120 & \begin{array}{c}110 \\ \mathrm{f} 1 \mathrm{(pom})\end{array} & \mathbf{8 0} & 70 & 60 & 50 & 40 & 30 & 20 & 10 & 0 & -10 & -20\end{array}$ ${ }^{1} \mathrm{H}$ and ${ }^{13} \mathrm{C}$ NMR spectra for compound $1 \mathrm{q}\left(400 \mathrm{MHz}, \mathrm{CDCl}_{3}\right.$ )<smiles>CCOC(=O)C(C[14C])C(=O)OCC</smiles>

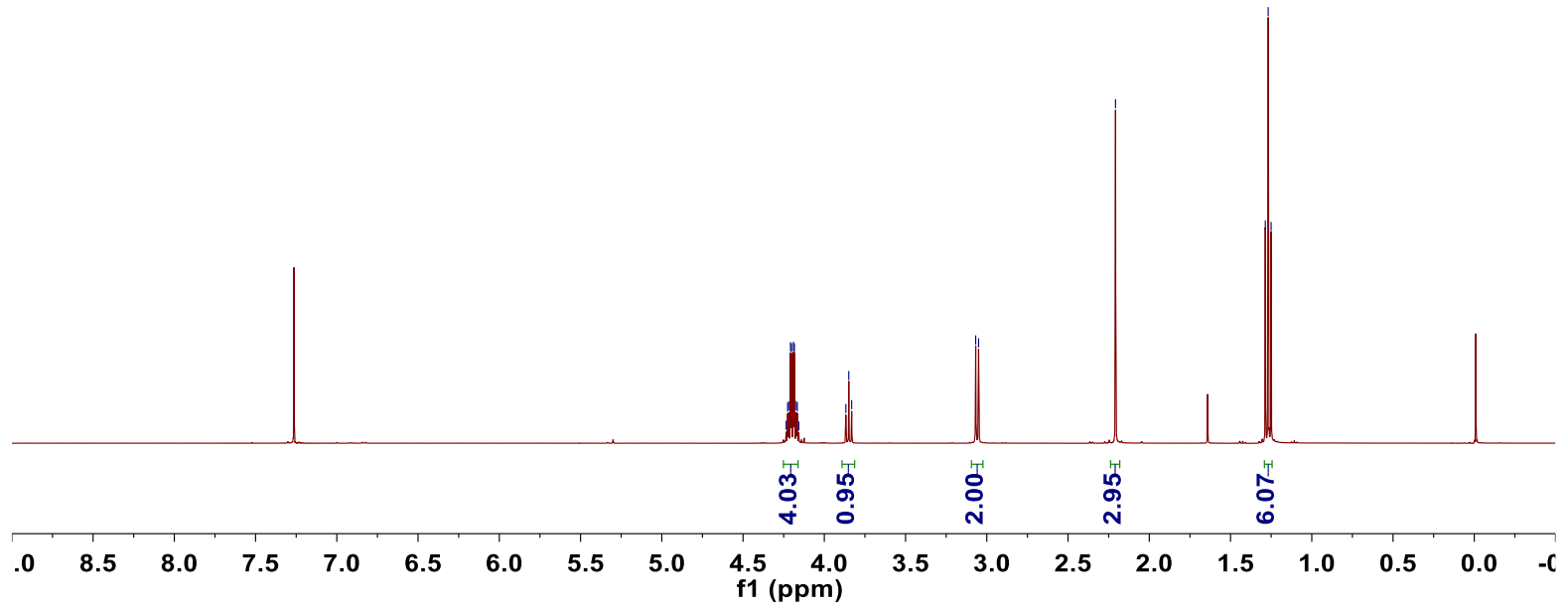

${ }^{1} \mathrm{H}$ NMR spectrum for compound $2 \mathrm{e}\left(400 \mathrm{MHz}, \mathrm{CDCl}_{3}\right)$ 


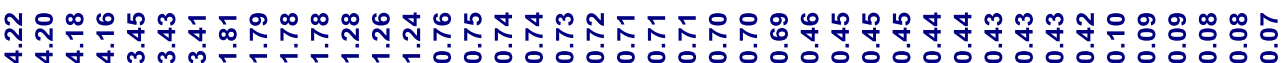<smiles>CCOC(=O)C(CC1CC1)C(=O)OCC</smiles>

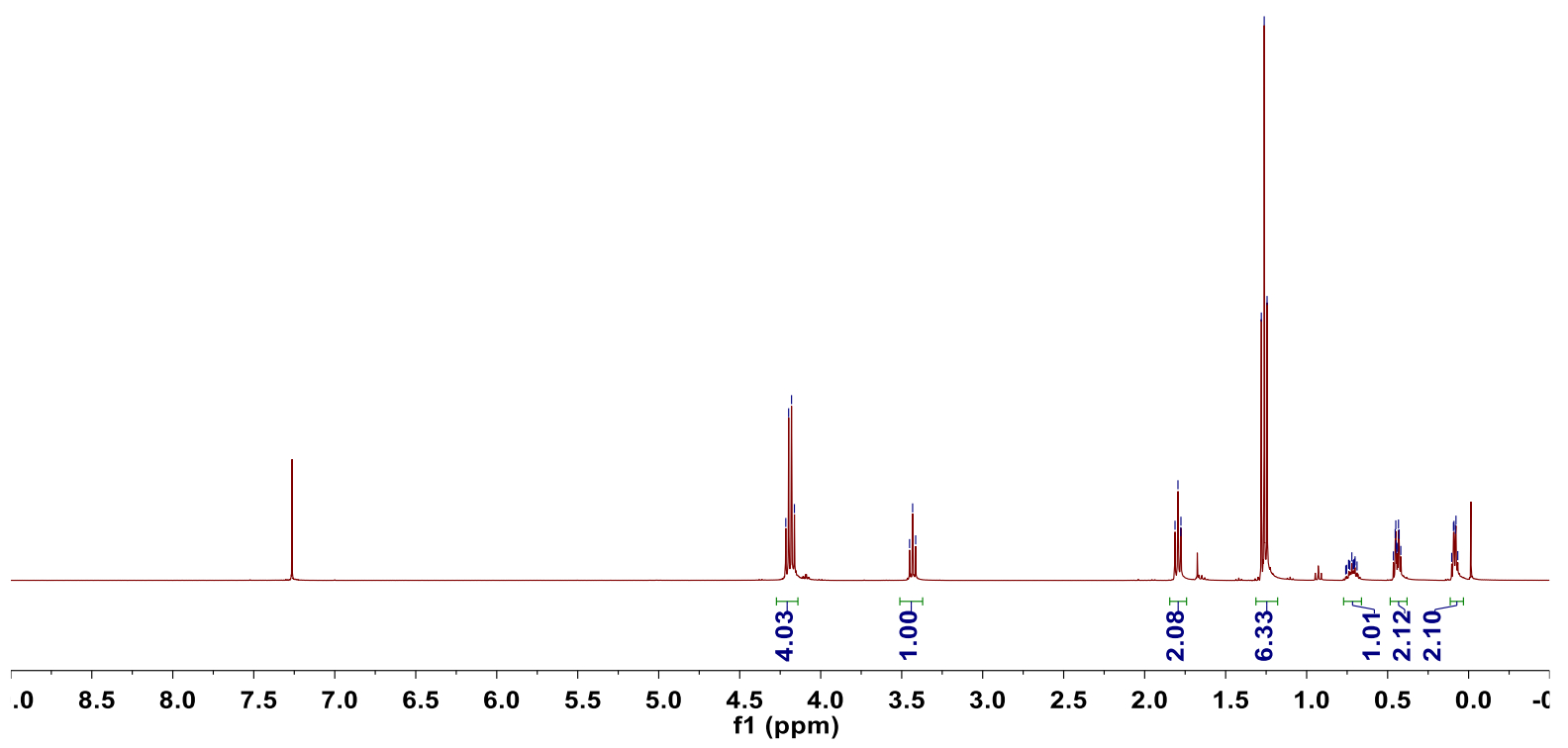

${ }^{1} \mathrm{H}$ NMR spectrum for compound $2 \mathrm{~g}\left(400 \mathrm{MHz}^{\mathrm{CDCl}}\right)_{3}$

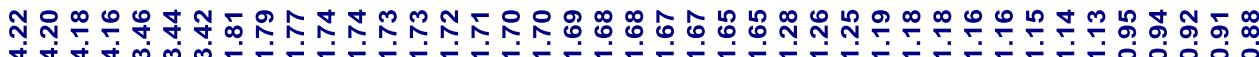<smiles>CCOC(=O)C(CC1CCCC1)C(=O)OCC</smiles>

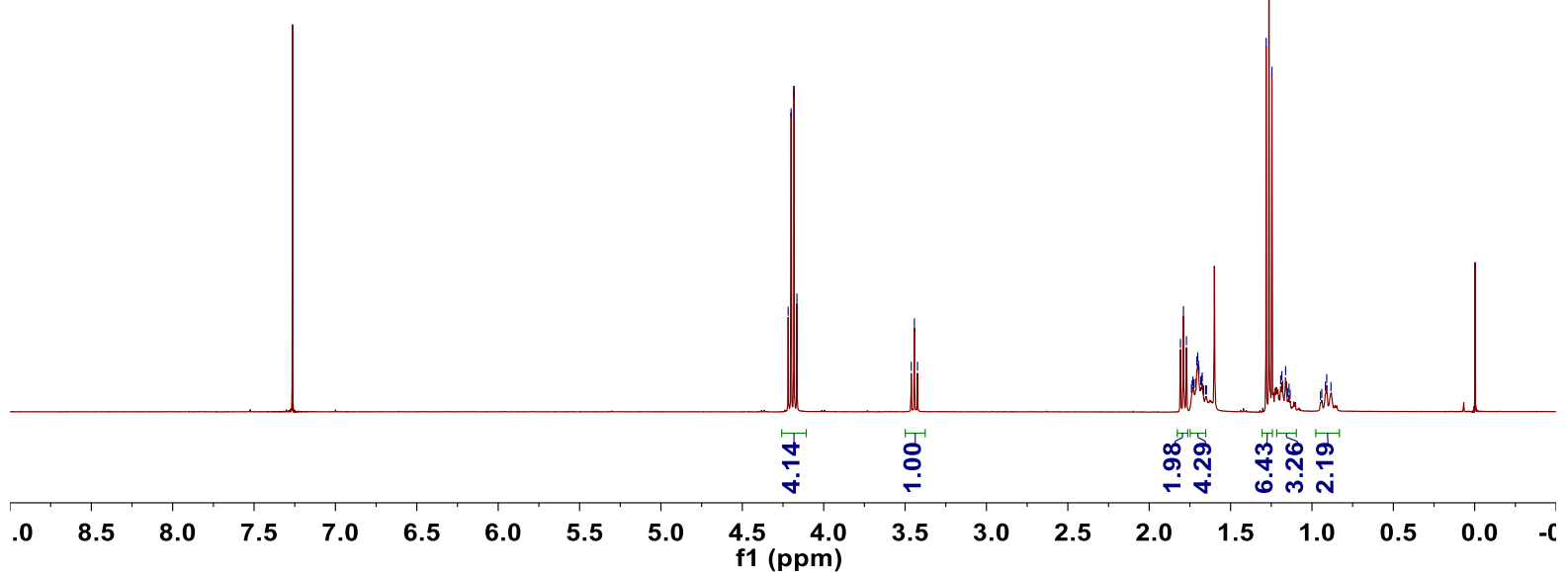

${ }^{1} \mathrm{H}$ NMR spectrum for compound $2 \mathrm{~h}\left(400 \mathrm{MHz}, \mathrm{CDCl}_{3}\right)$ 


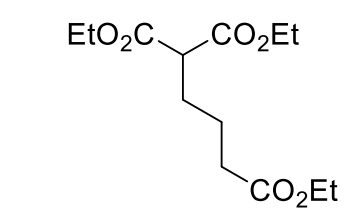

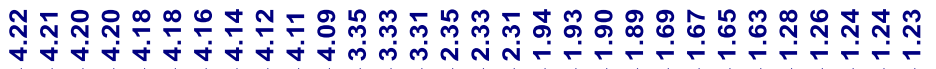

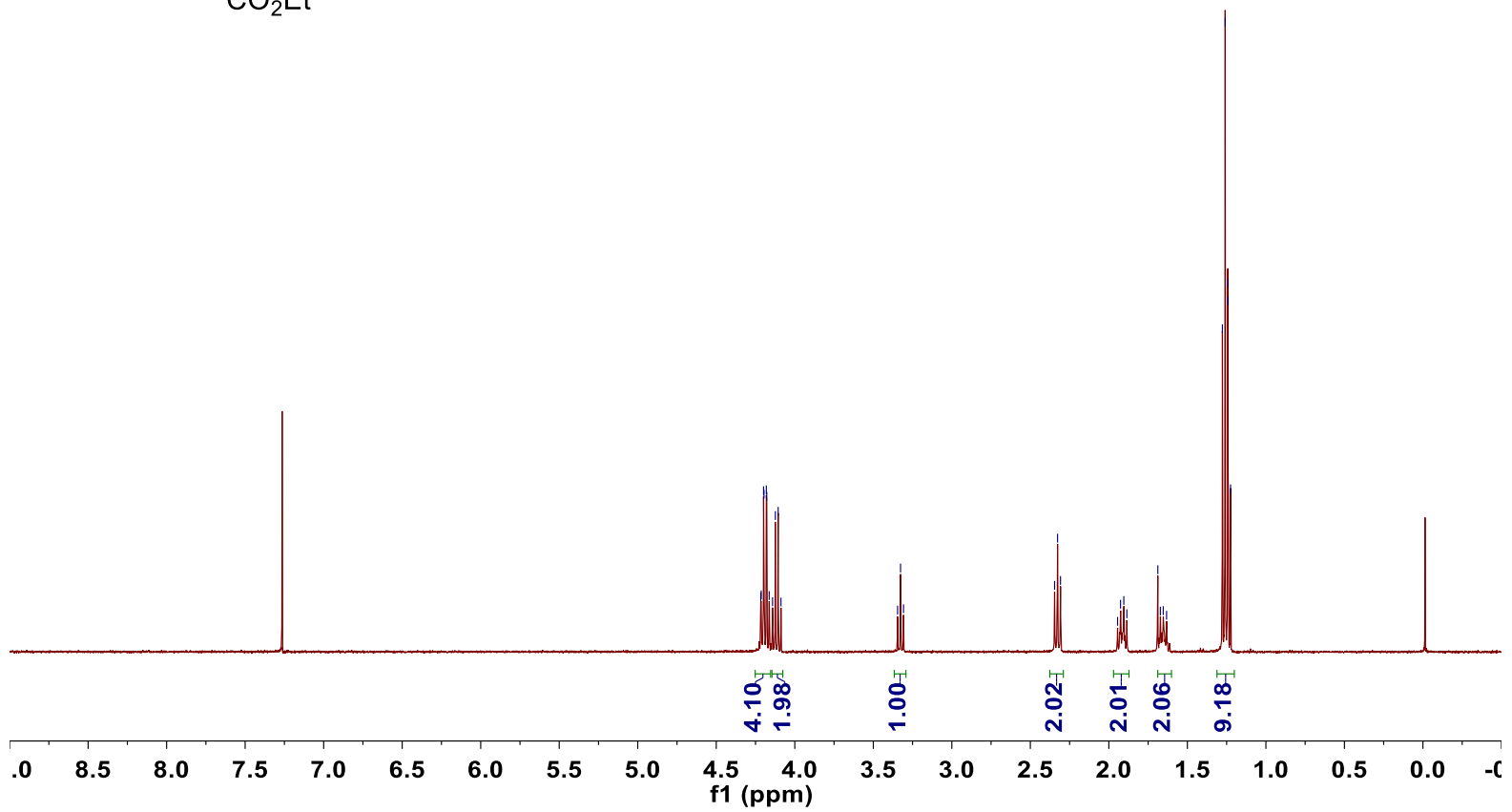

${ }^{1} \mathrm{H}$ NMR spectrum for compound 2i $\left(400 \mathrm{MHz}, \mathrm{CDCl}_{3}\right)$

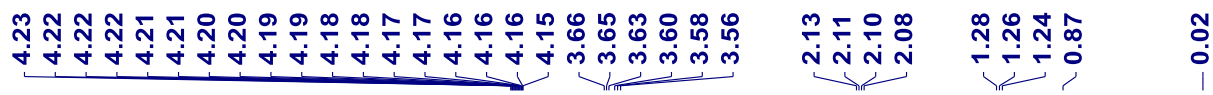<smiles>CCOC(CCO[SbH3])C(OCC)OCC</smiles>

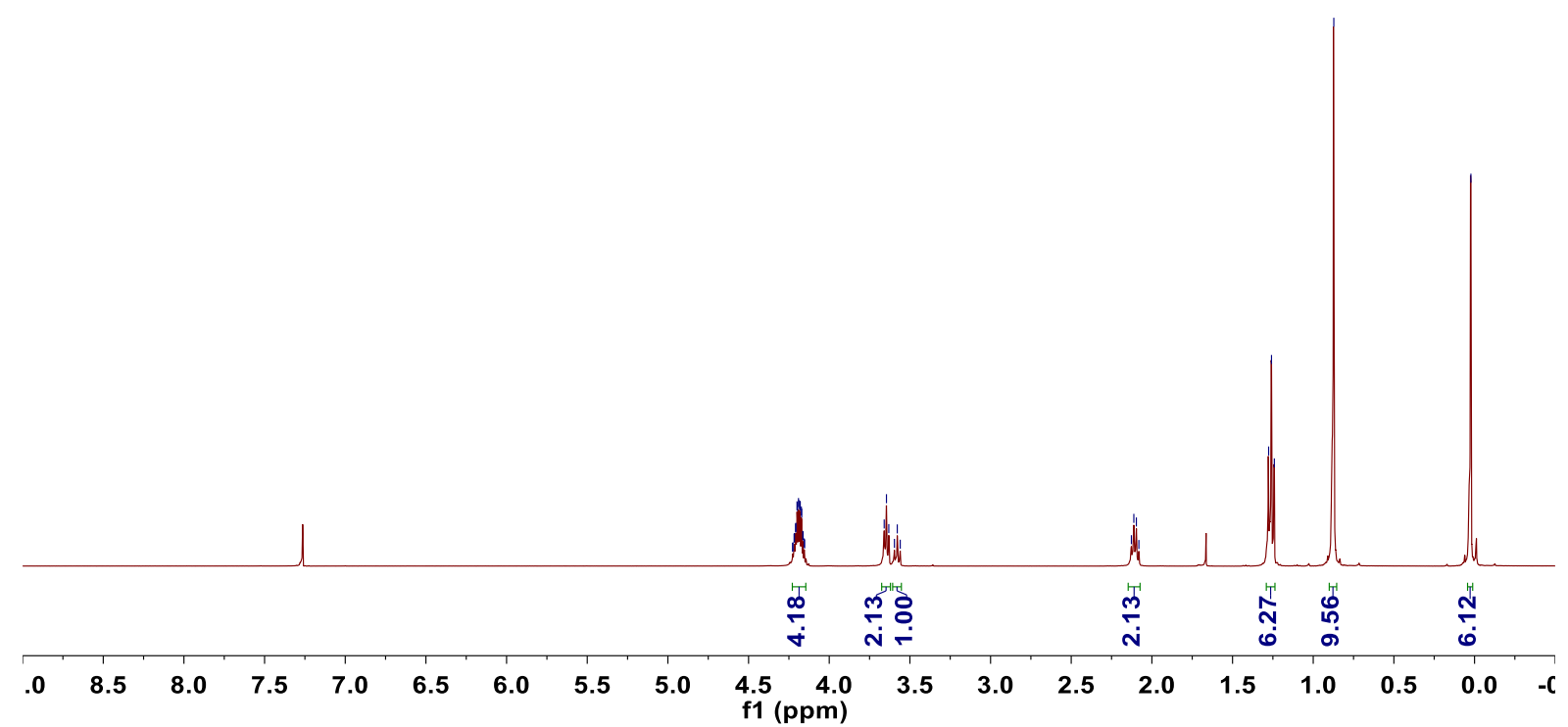

${ }^{1} \mathrm{H}$ NMR spectrum for compound 2j $\left(400 \mathrm{MHz}, \mathrm{CDCl}_{3}\right)$ 


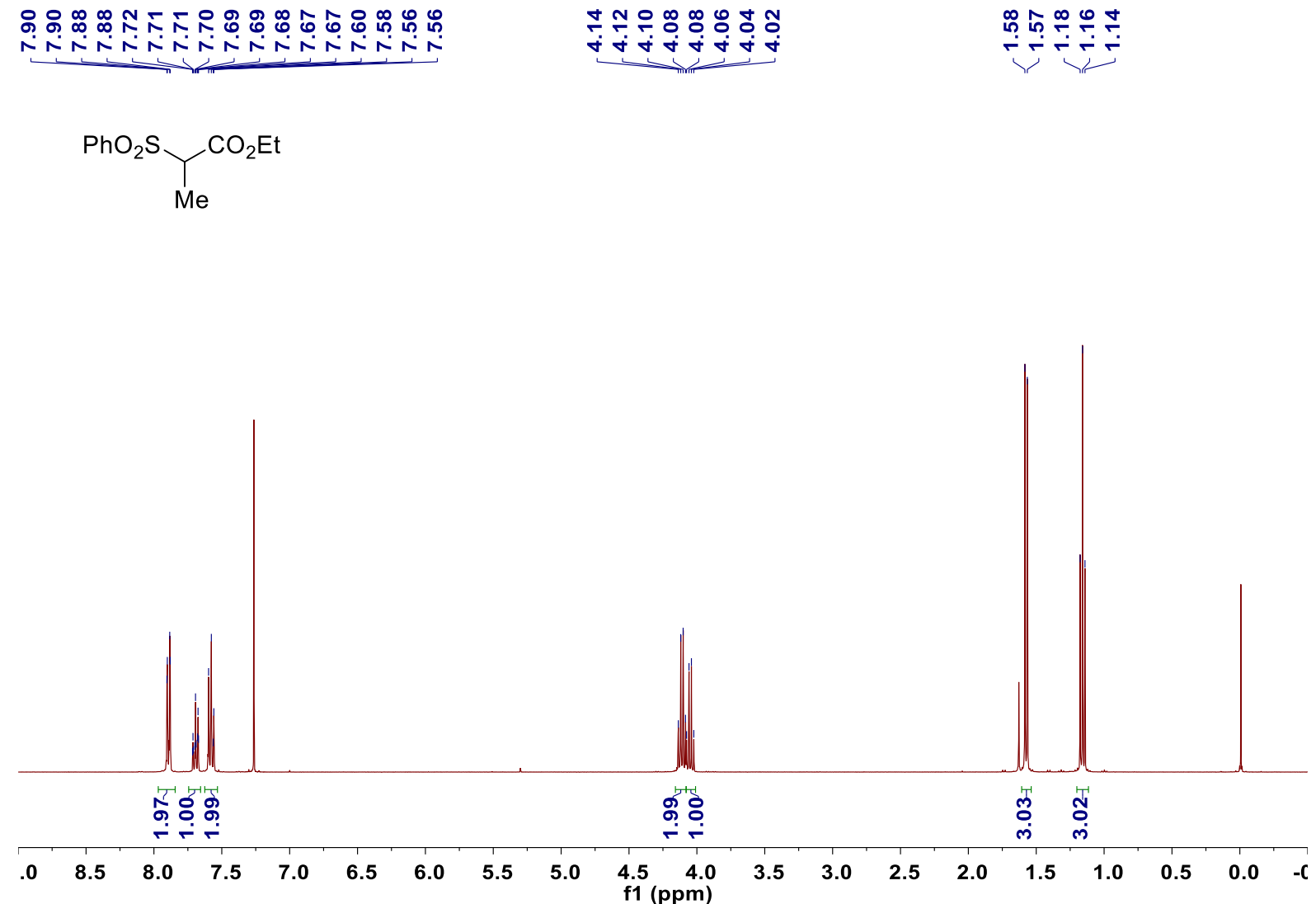

${ }^{1} \mathrm{H}$ NMR spectrum for compound $2 \mathrm{~m}\left(400 \mathrm{MHz}, \mathrm{CDCl}_{3}\right)$
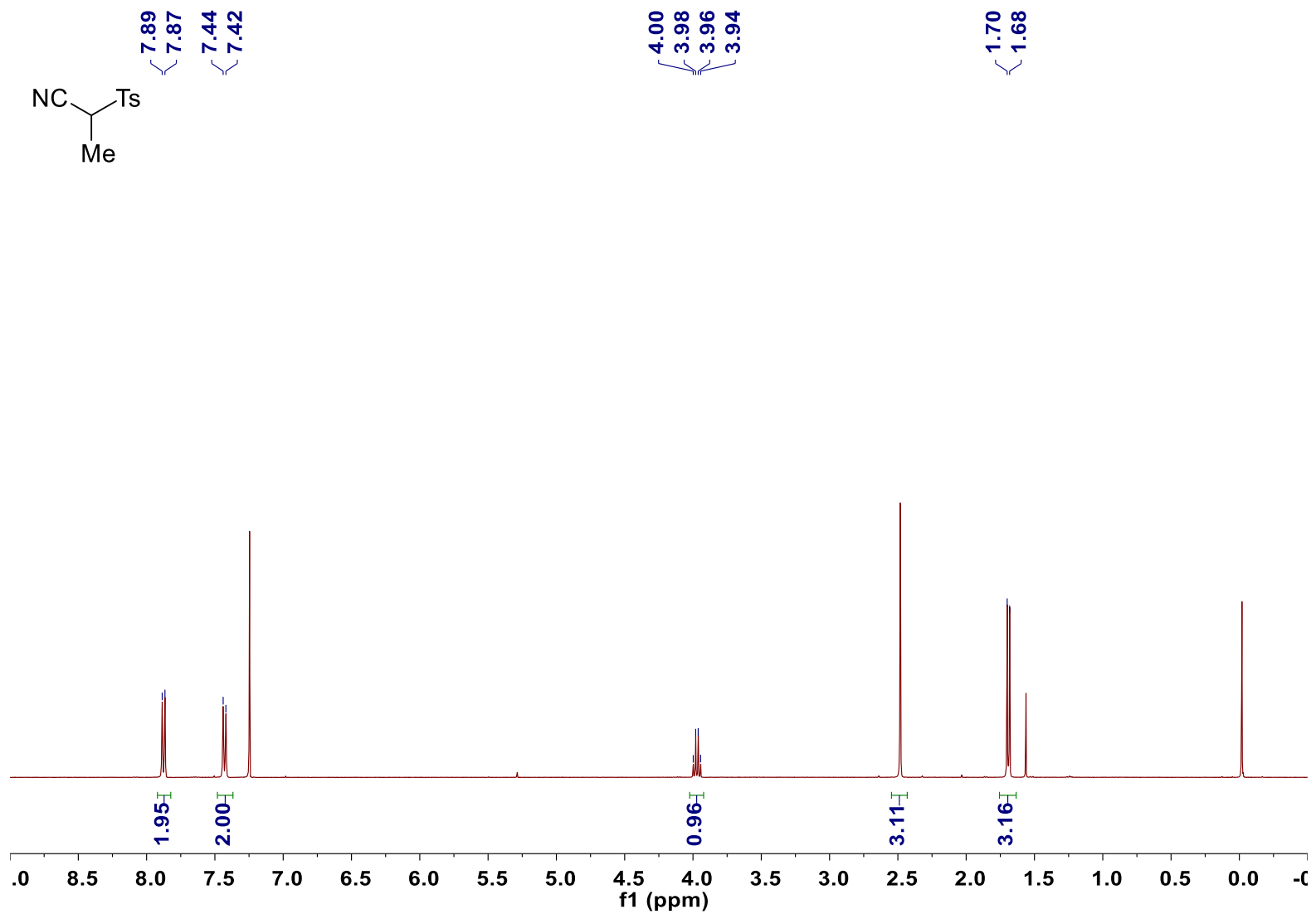

${ }^{1} \mathrm{H}$ NMR spectrum for compound $2 \mathrm{n}\left(400 \mathrm{MHz}, \mathrm{CDCl}_{3}\right)$ 


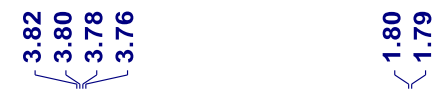

${ }^{\mathrm{NC}} \mathrm{Me}_{\mathrm{Me}}^{\mathrm{CN}}$

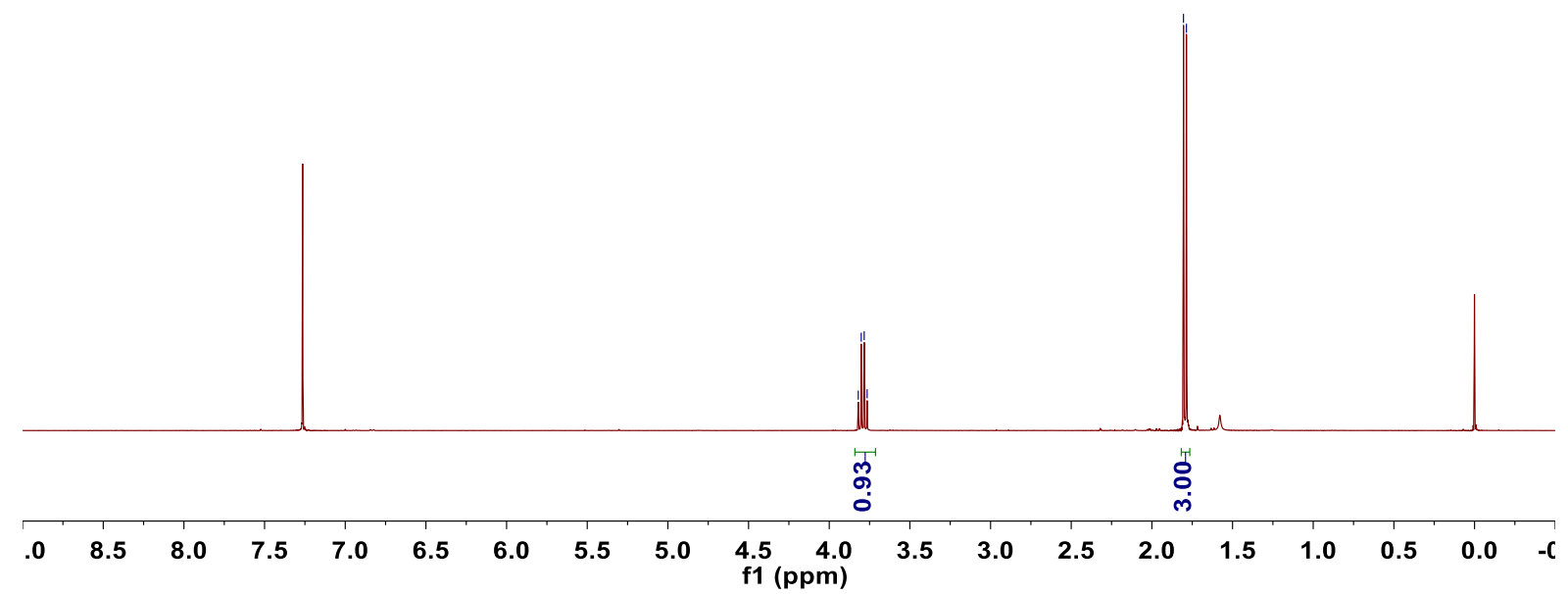

${ }^{1} \mathrm{H}$ NMR spectrum for compound $20\left(400 \mathrm{MHz}, \mathrm{CDCl}_{3}\right)$

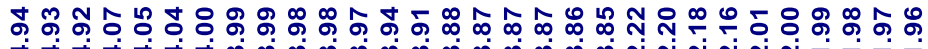<smiles>N#CC(C#N)CCC1OCCO1</smiles>

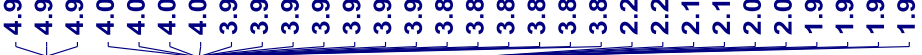

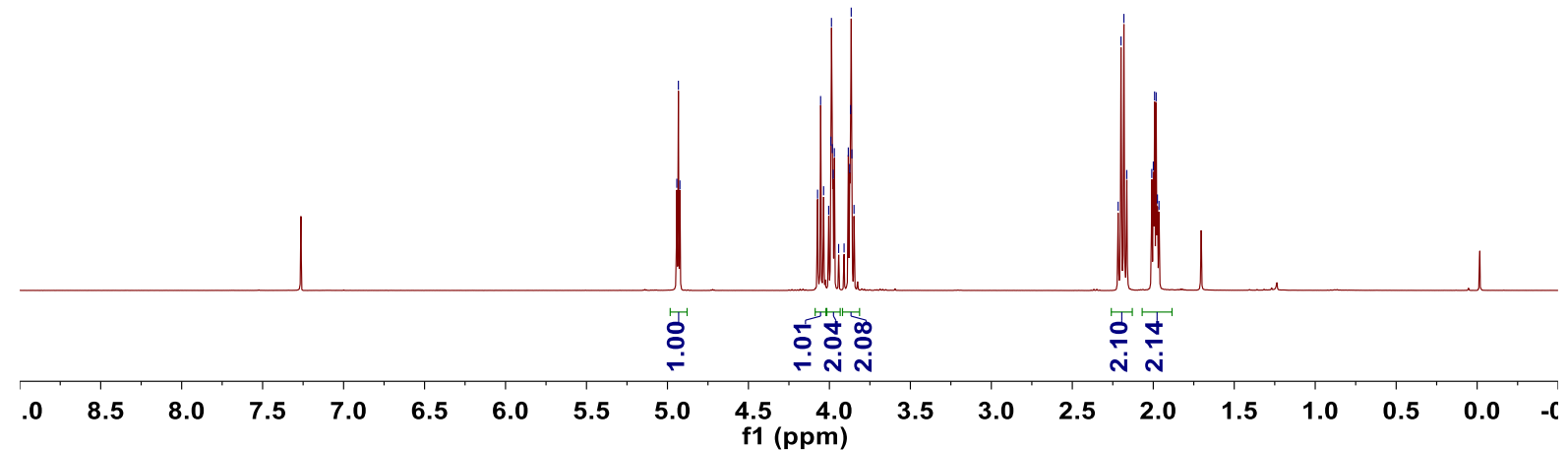




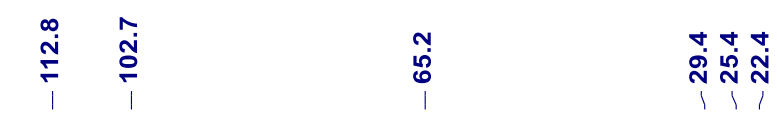

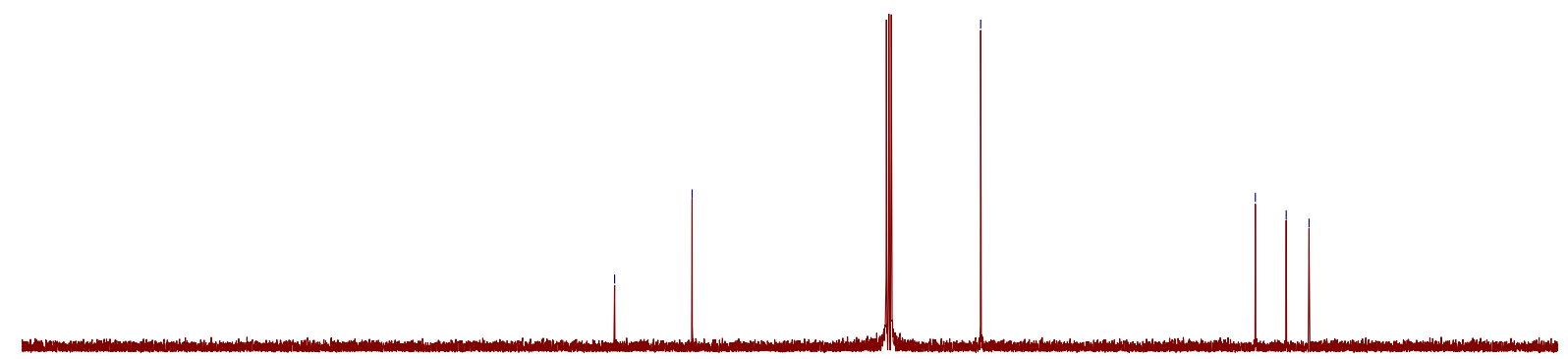

$\begin{array}{llllllllllllllllllll}180 & 170 & 160 & 150 & 140 & 130 & 120 & 110 & 100 \underset{f 1}{90} & 80 & 70 & 60 & 50 & 40 & 30 & 20 & 10 & 0 & -\end{array}$

${ }^{1} \mathrm{H}$ and ${ }^{13} \mathrm{C}$ NMR spectra for compound $2 \mathrm{p}\left(400 \mathrm{MHz}, \mathrm{CDCl}_{3}\right)$

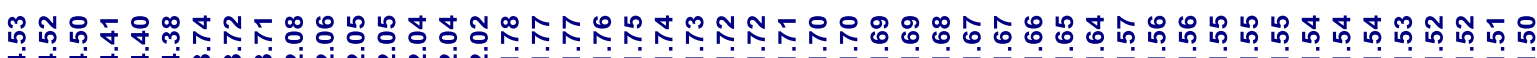

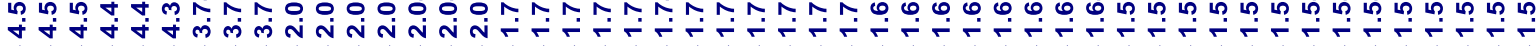<smiles>N#CC(C#N)CCCCCF</smiles>

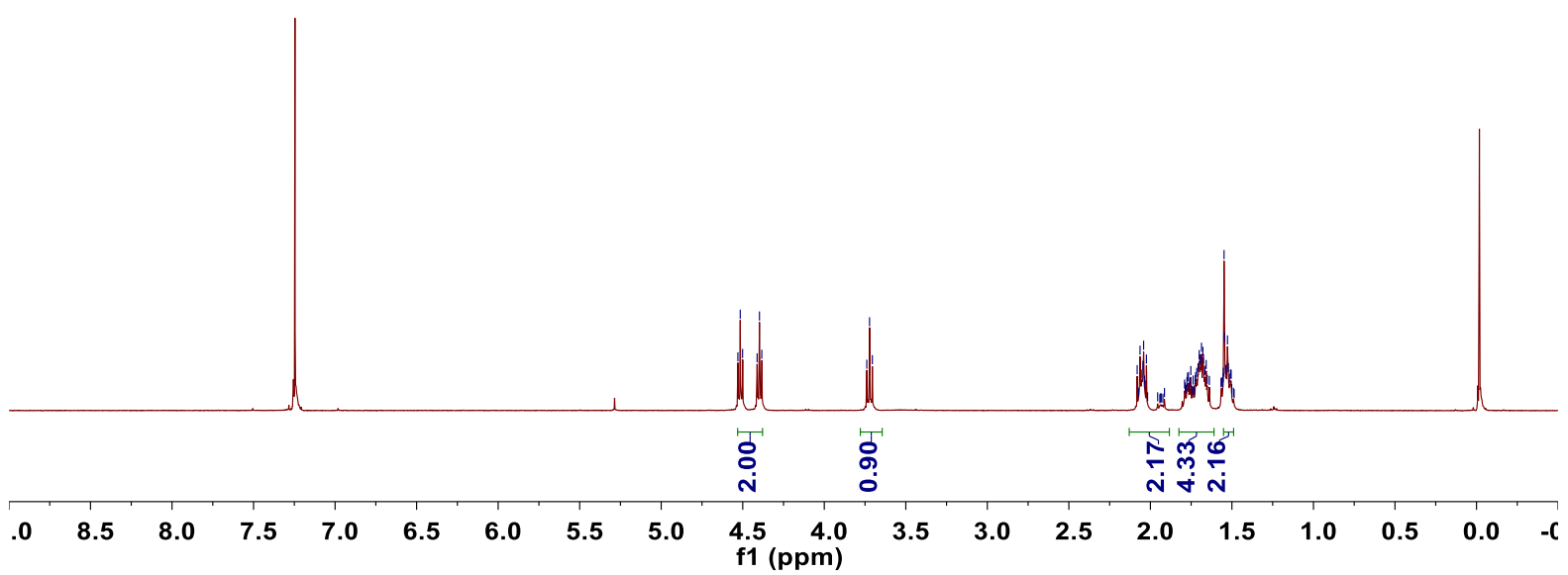




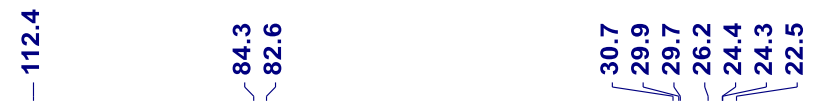

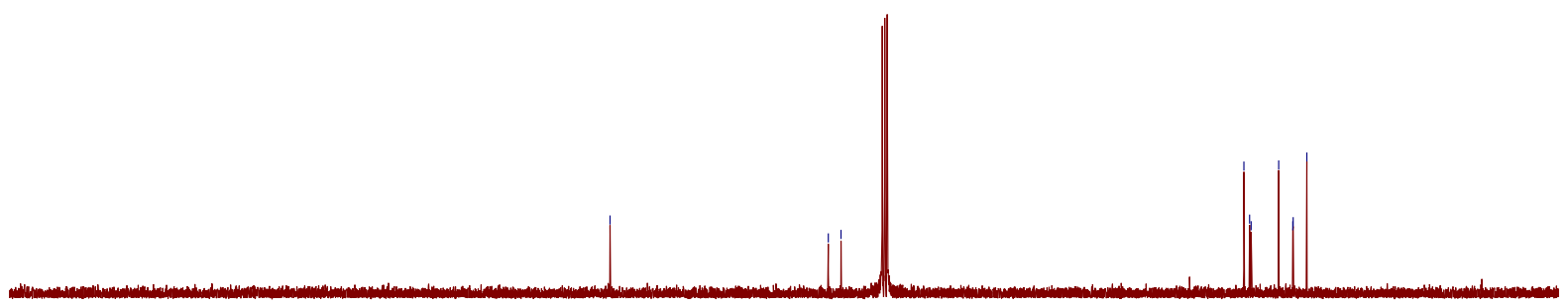

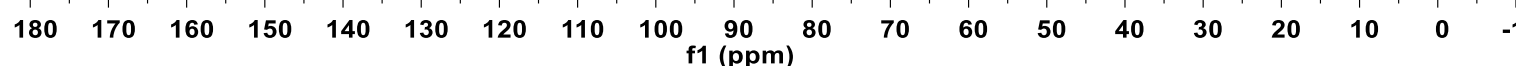

$\stackrel{\circ}{\circ}$

$\begin{array}{llllllllllllllllllllllll}100 & 80 & 60 & 40 & 20 & 0 & -20 & -40 & -60 & -80 & -100 & -120 & -140 & -160 & -180 & -200 & -220 & -240 & -260 & -280 & -30 C\end{array}$ ${ }^{1} \mathrm{H},{ }^{13} \mathrm{C}$ and ${ }^{19} \mathrm{~F}$ NMR spectra for compound $2 \mathrm{q}\left(400 \mathrm{MHz}, \mathrm{CDCl}_{3}\right)$ 


\section{NMR spectra of all the products}
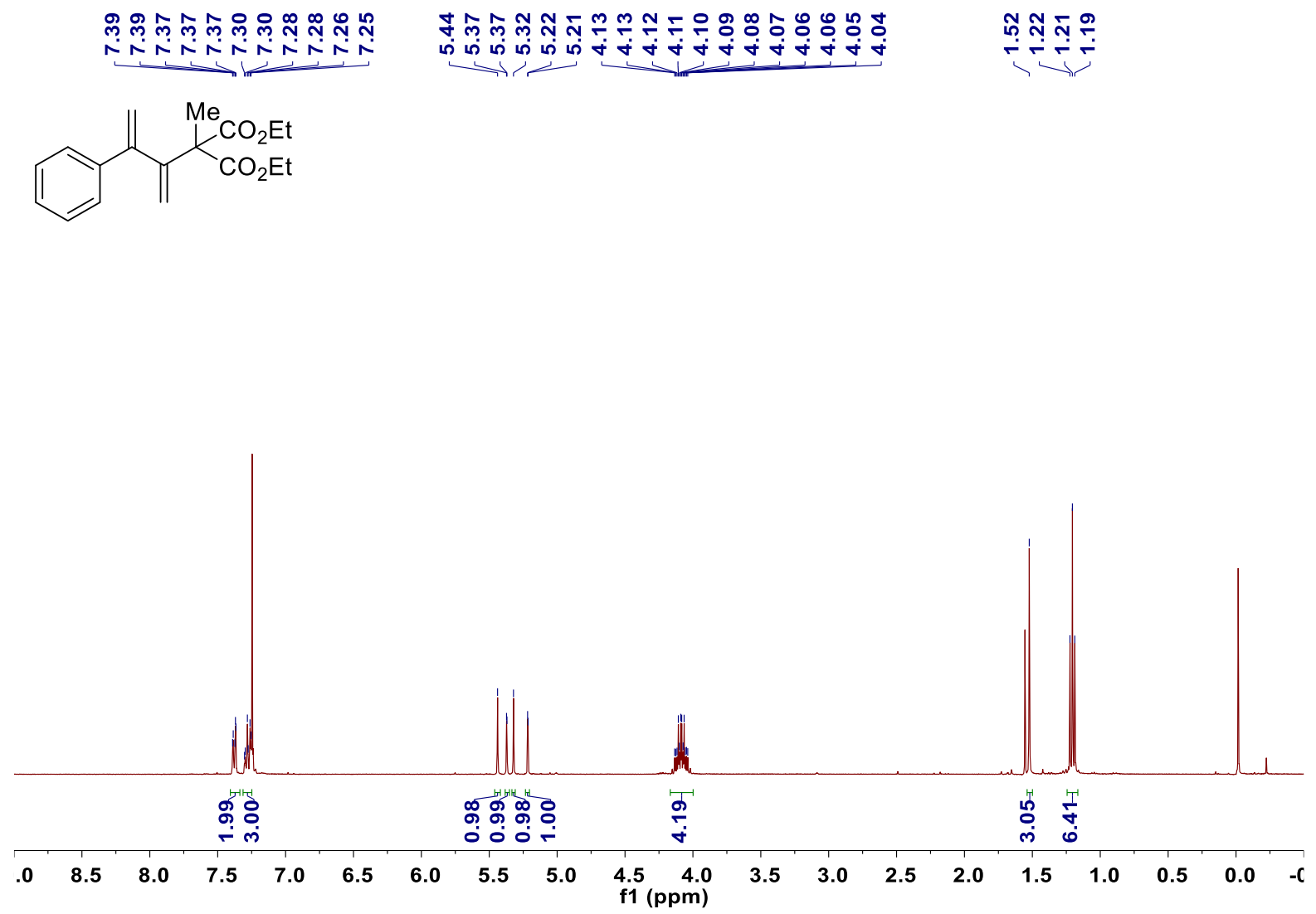

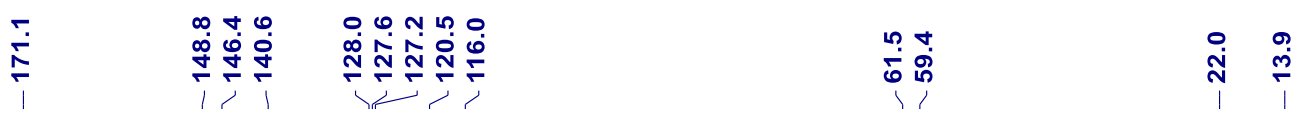

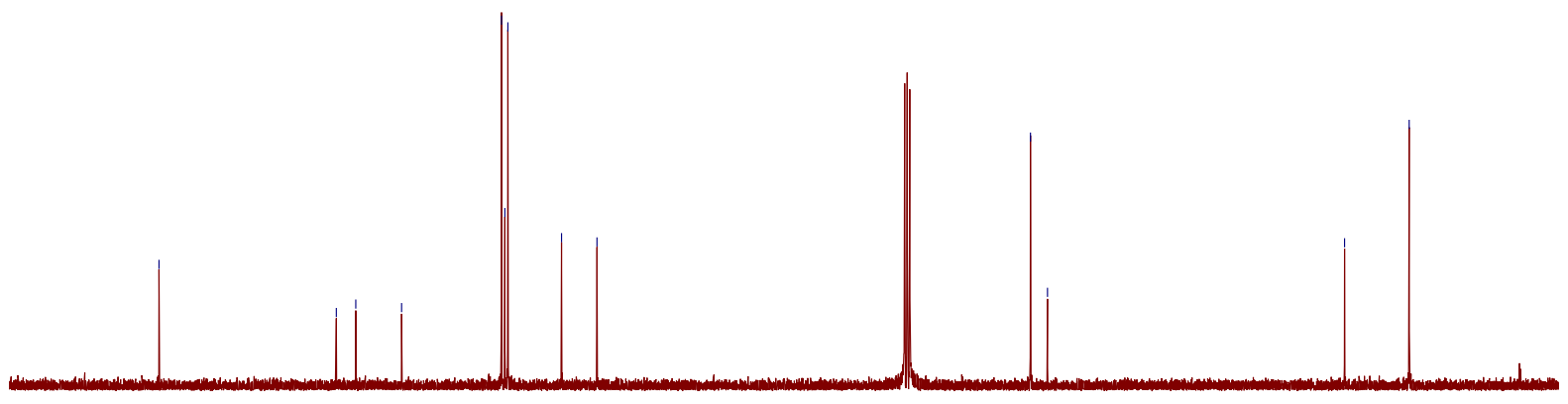

$\begin{array}{lllllllllll}90 & 180 & 170 & 160 & 150 & 140 & 130 & 120 & 110 & 100 & \begin{array}{c}90 \\ \mathrm{f} 1(\mathrm{ppm})\end{array}\end{array}$

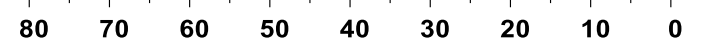

${ }^{1} \mathrm{H}$ and ${ }^{13} \mathrm{C}$ NMR spectra for product $3 a a\left(400 \mathrm{MHz}, \mathrm{CDCl}_{3}\right)$ 


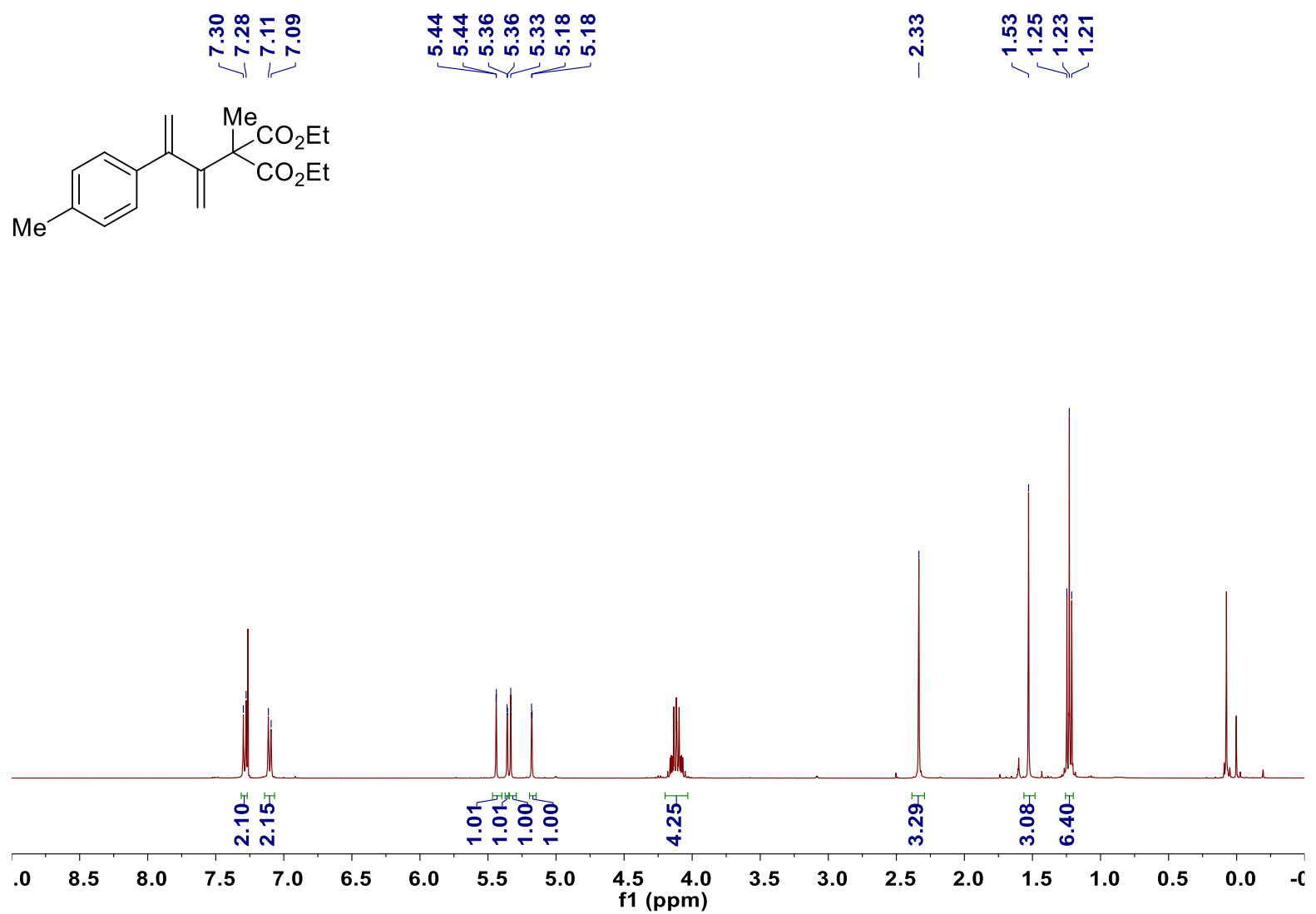

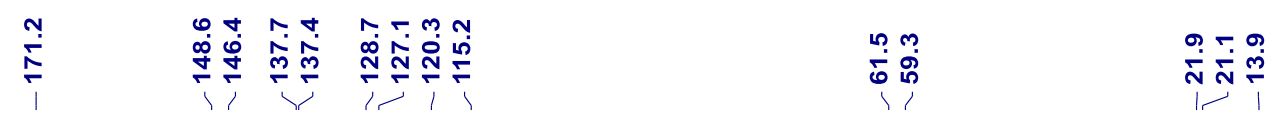

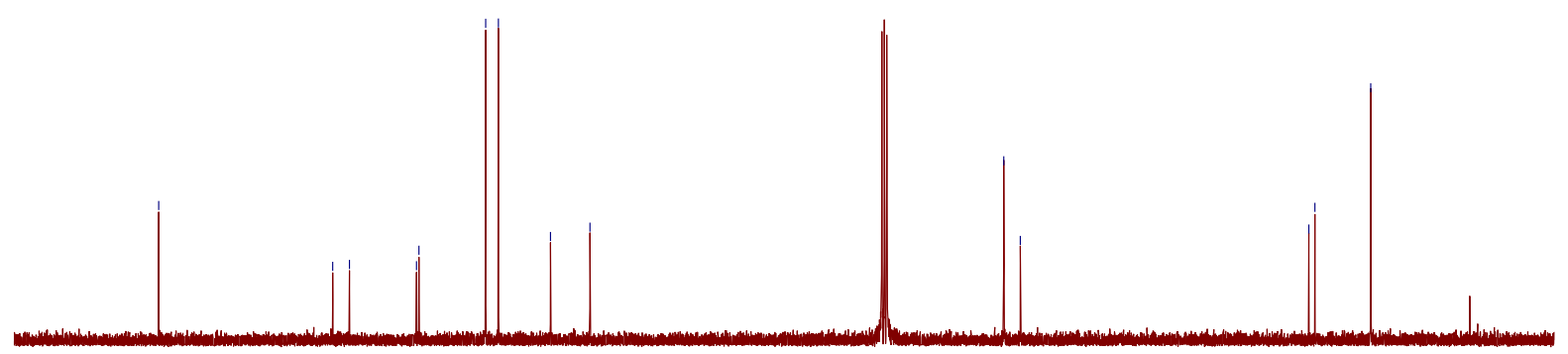

$\begin{array}{lllllllllllllllllllllll}90 & 180 & 170 & 160 & 150 & 140 & 130 & 120 & 110 & 100 & 90 & 80 & 70 & 60 & 50 & 40 & 30 & 20 & 10 & 0 & -\end{array}$

${ }^{1} \mathrm{H}$ and ${ }^{13} \mathrm{C}$ NMR spectra for product $3 \mathrm{ba}\left(400 \mathrm{MHz}, \mathrm{CDCl}_{3}\right)$ 


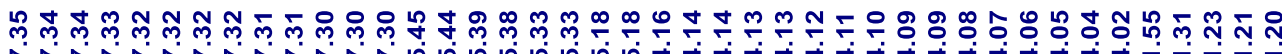

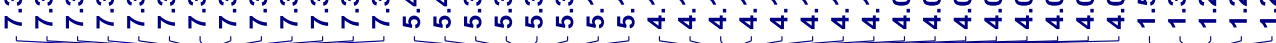<smiles>C=C(C(=C)C(C)(C(C)=O)C(=O)OCC)c1ccc(C(C)(C)C)cc1</smiles>

t-Bu

$\mathrm{CO}_{2} \mathrm{Et}$

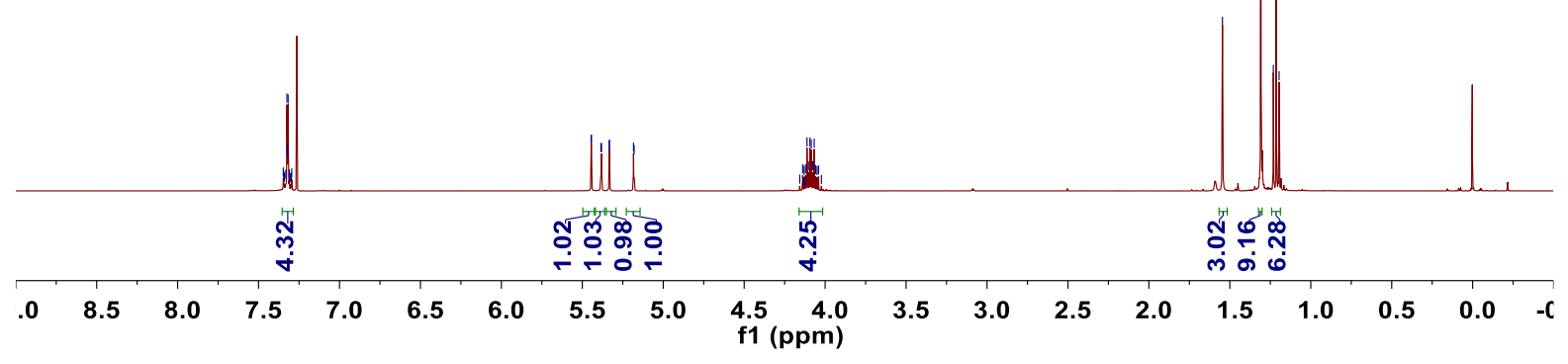

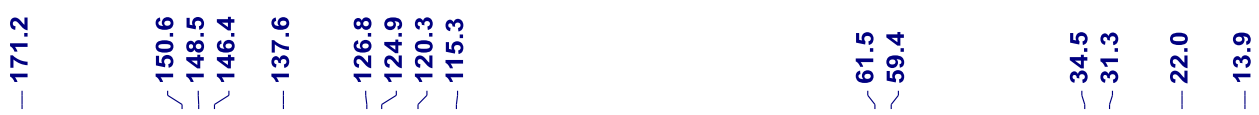

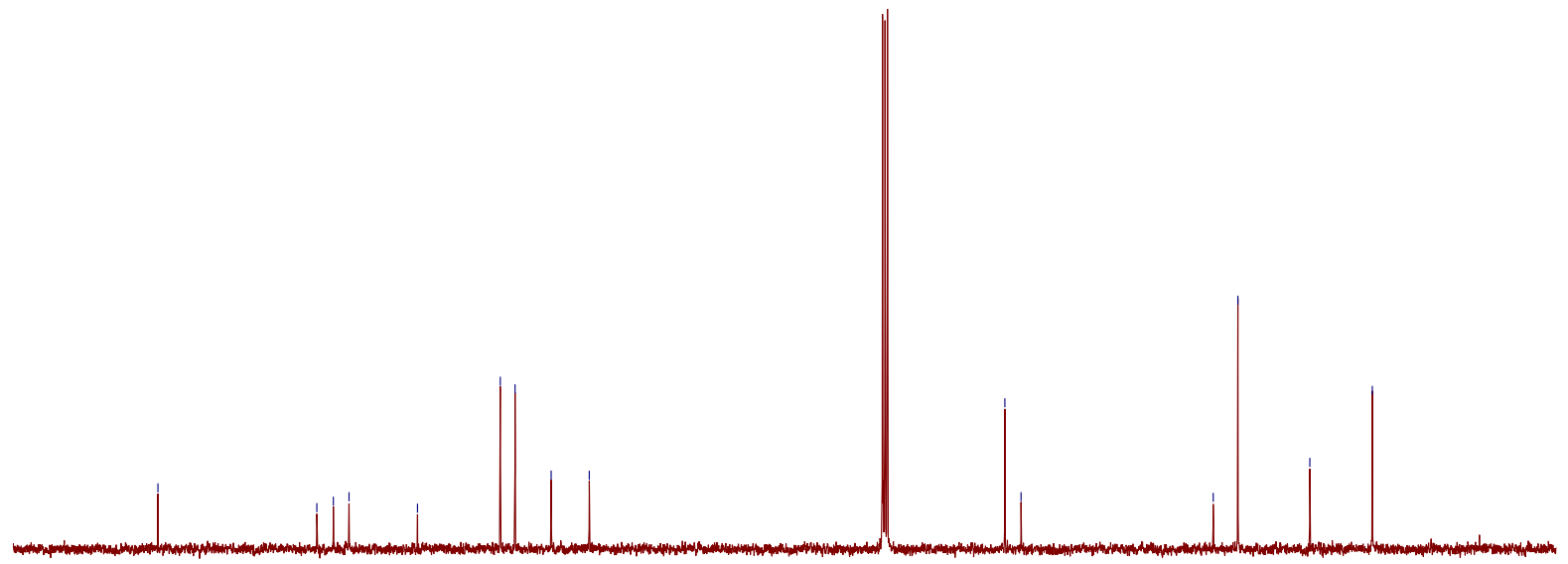

$\begin{array}{lllllllllllllllllllll}90 & 180 & 170 & 160 & 150 & 140 & 130 & 120 & 110 & 100 & \underset{\mathrm{f} 1}{(\mathrm{ppm})} & 80 & 70 & 60 & 50 & 40 & 30 & 20 & 10 & 0 & -\end{array}$

${ }^{1} \mathrm{H}$ and ${ }^{13} \mathrm{C}$ NMR spectra for product $3 \mathrm{ca}\left(400 \mathrm{MHz}, \mathrm{CDCl}_{3}\right)$ 


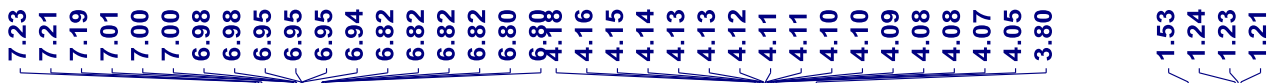<smiles>C=C(C(=C)C(C)(C)OCC)c1cccc(OC)c1</smiles>

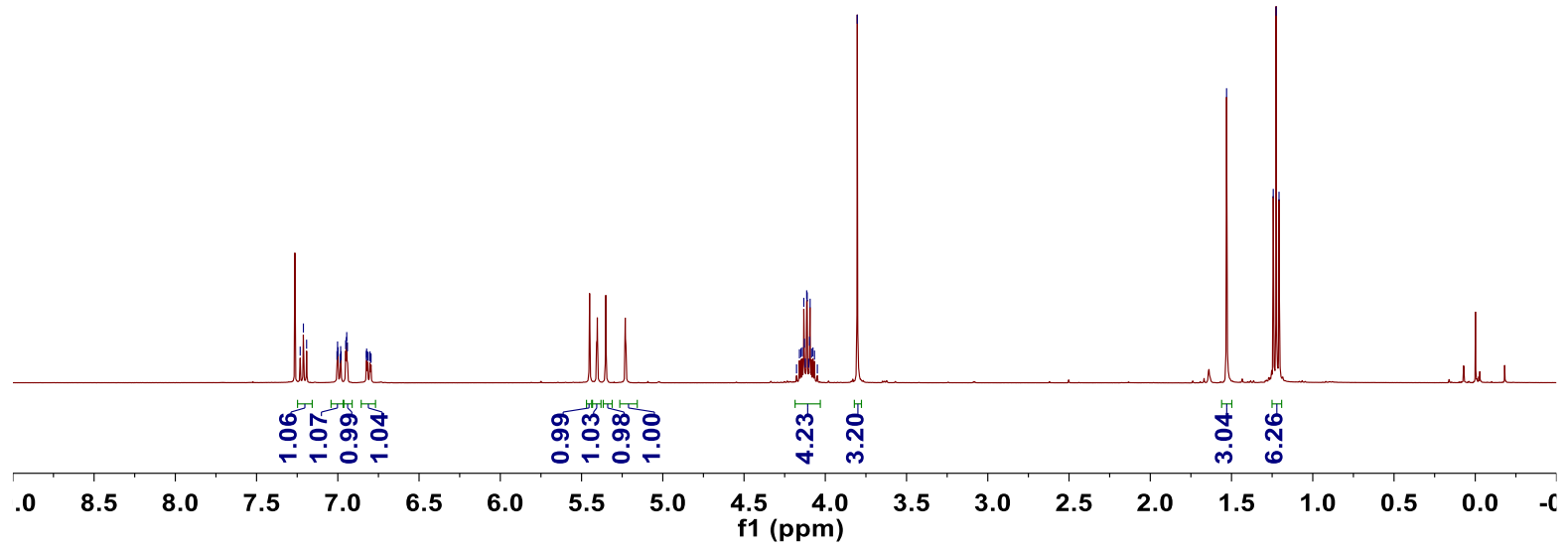

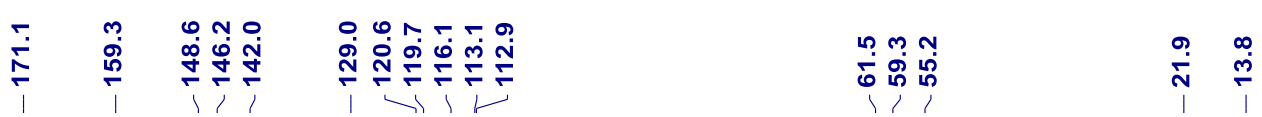

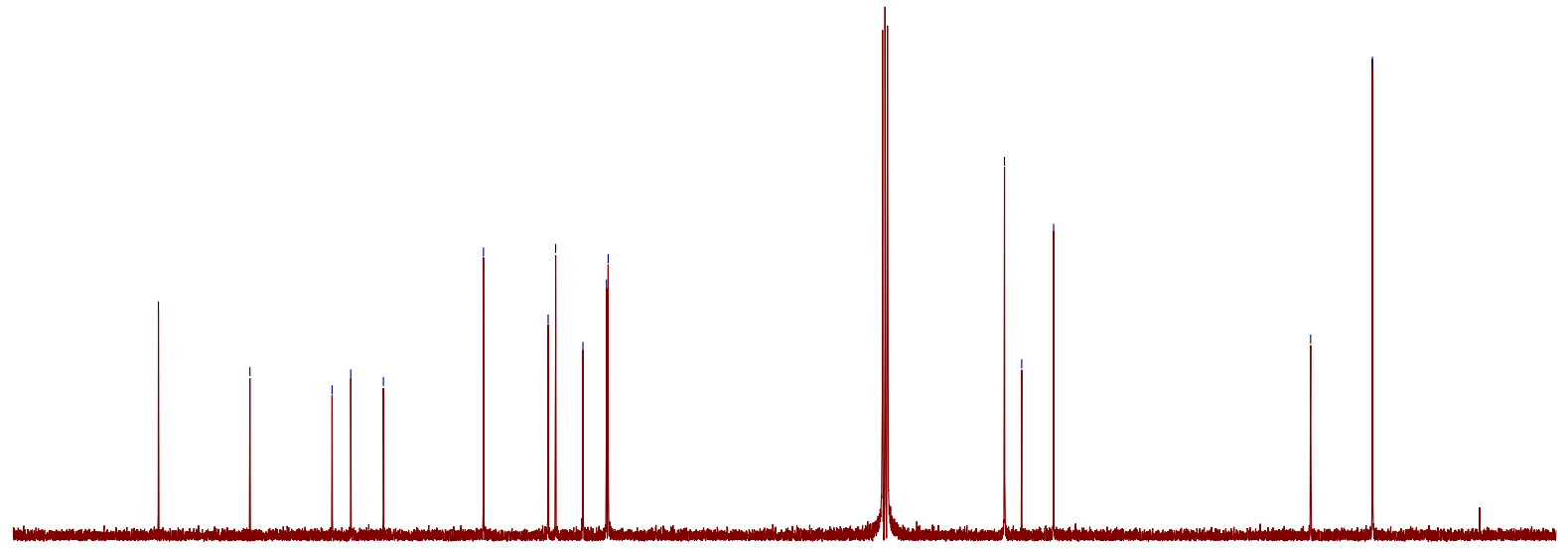

$\begin{array}{lllllllllllllllllllll}180 & 170 & 160 & 150 & 140 & 130 & 120 & 110 & 100 & 90 & 80 & 70 & 60 & 50 & 40 & 30 & 20 & 10 & 0 & -\end{array}$

${ }^{1} \mathrm{H}$ and ${ }^{13} \mathrm{C}$ NMR spectra for product $3 \mathrm{da}\left(400 \mathrm{MHz}, \mathrm{CDCl}_{3}\right)$ 


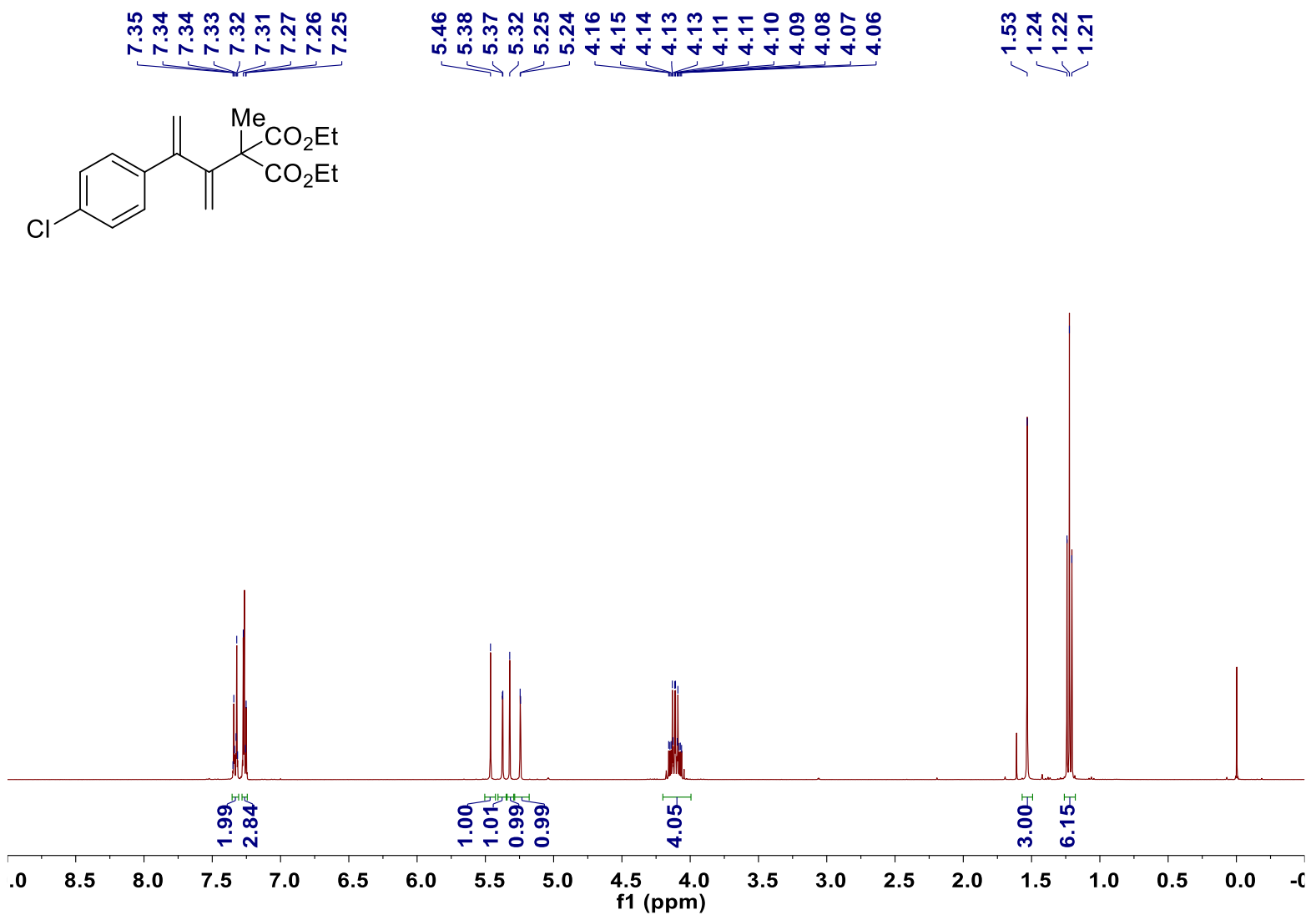

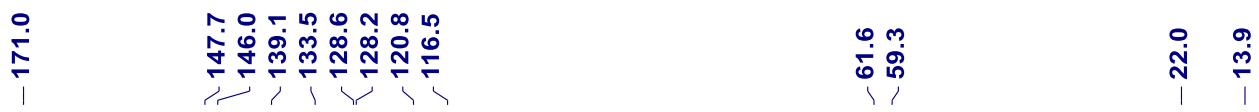

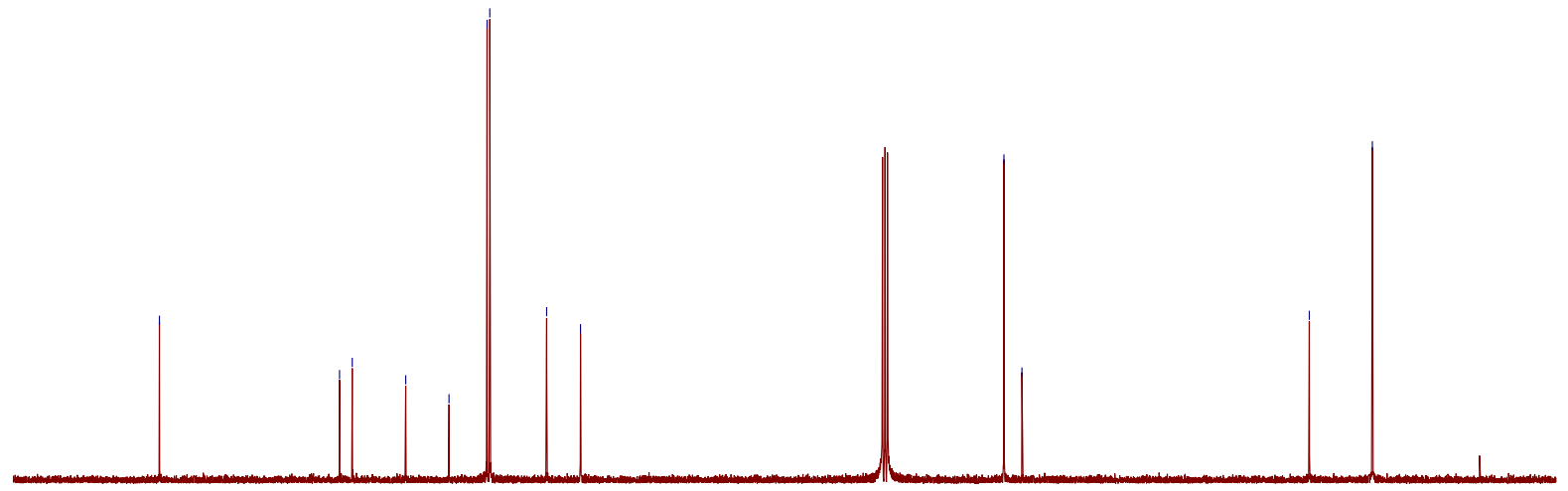

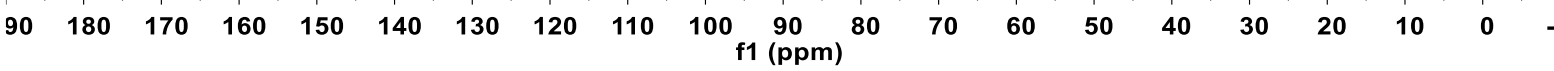

${ }^{1} \mathrm{H}$ and ${ }^{13} \mathrm{C}$ NMR spectra for product 3 ea $\left(400 \mathrm{MHz}, \mathrm{CDCl}_{3}\right)$ 


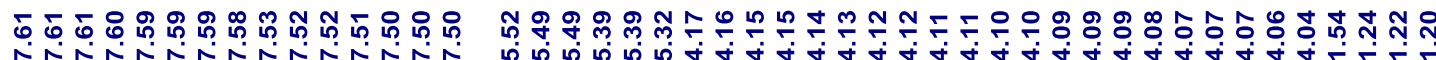

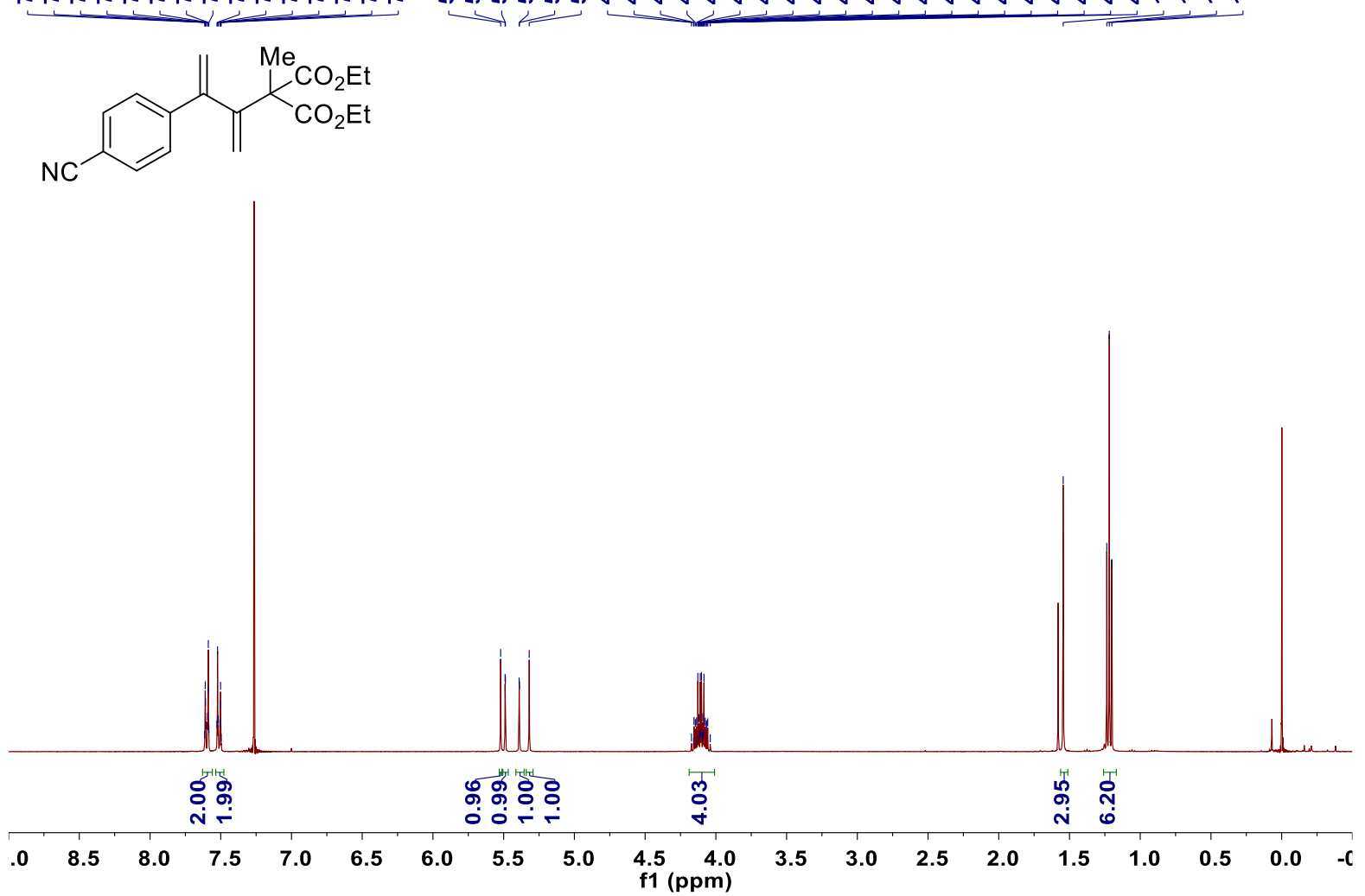

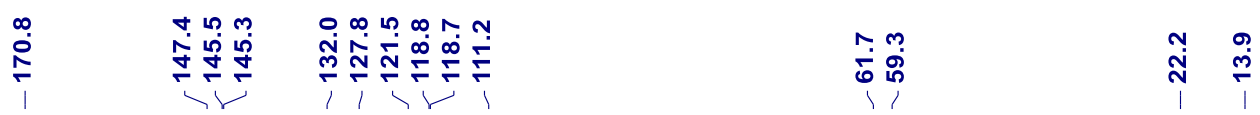

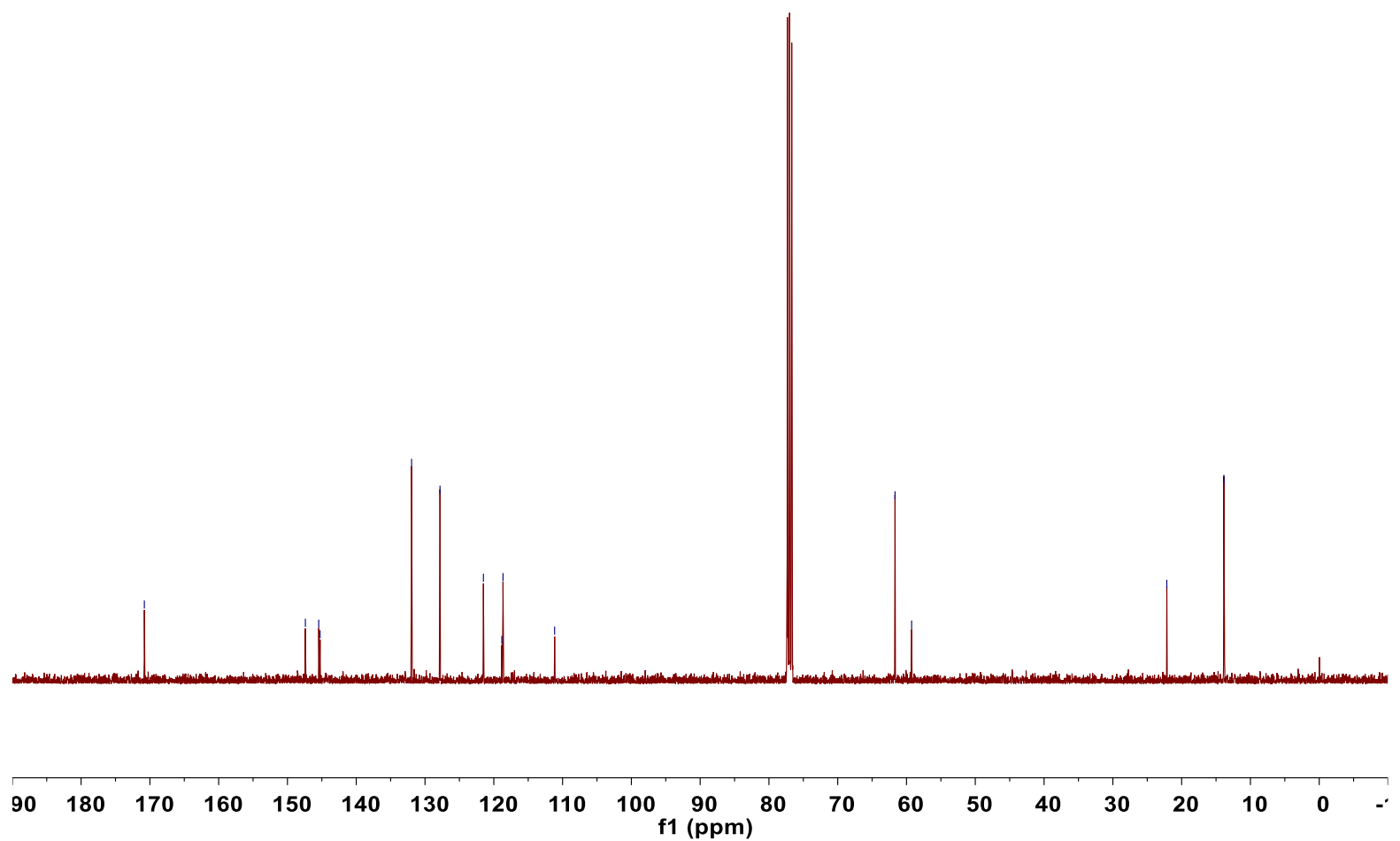

${ }^{1} \mathrm{H}$ and ${ }^{13} \mathrm{C}$ NMR spectra for product $3 f a\left(400 \mathrm{MHz}, \mathrm{CDCl}_{3}\right)$ 
กิ่

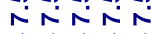

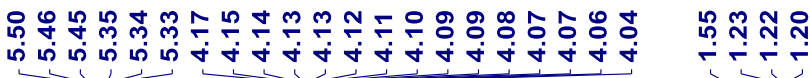

侻

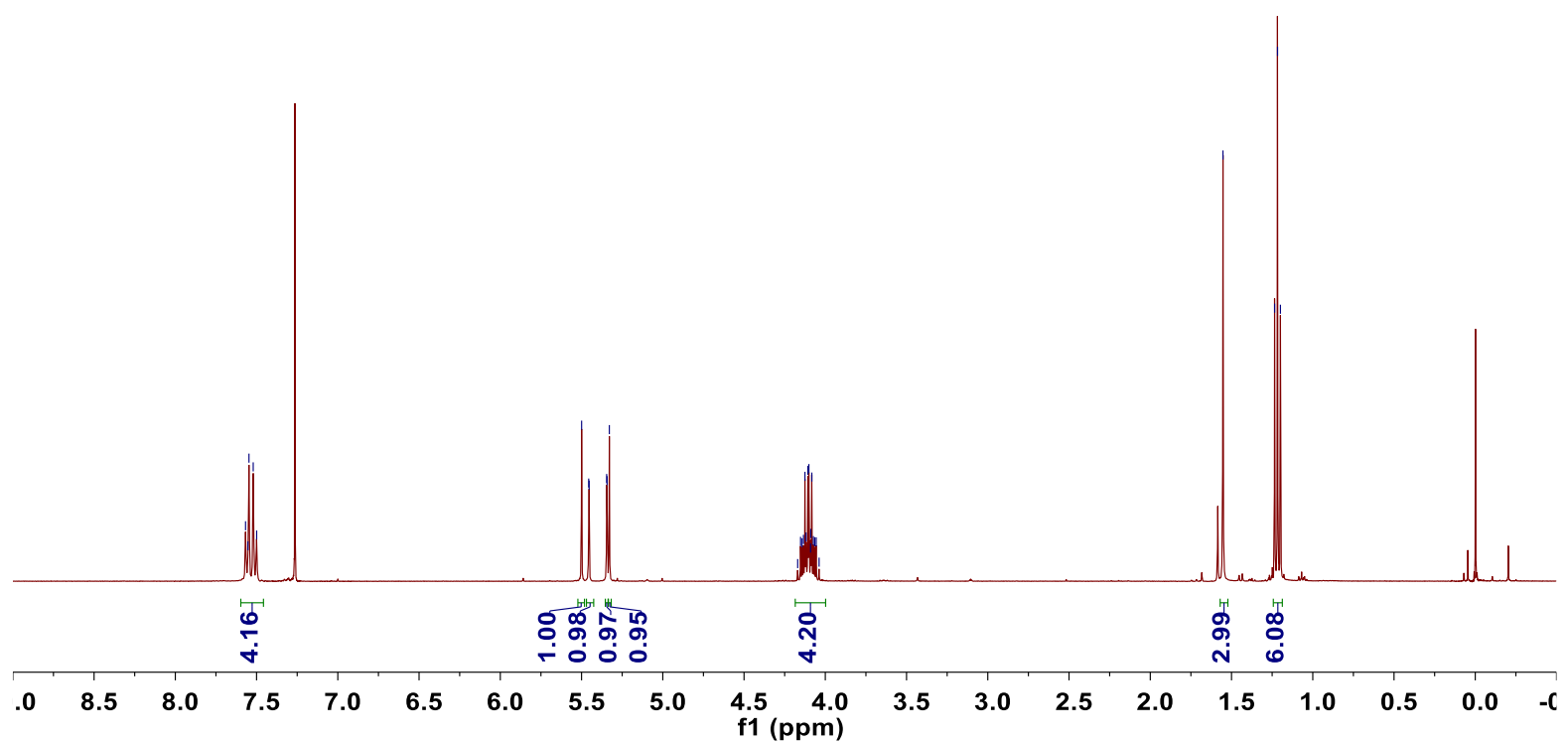

r. $\quad$ mo

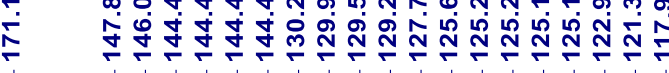

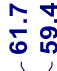

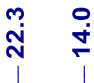

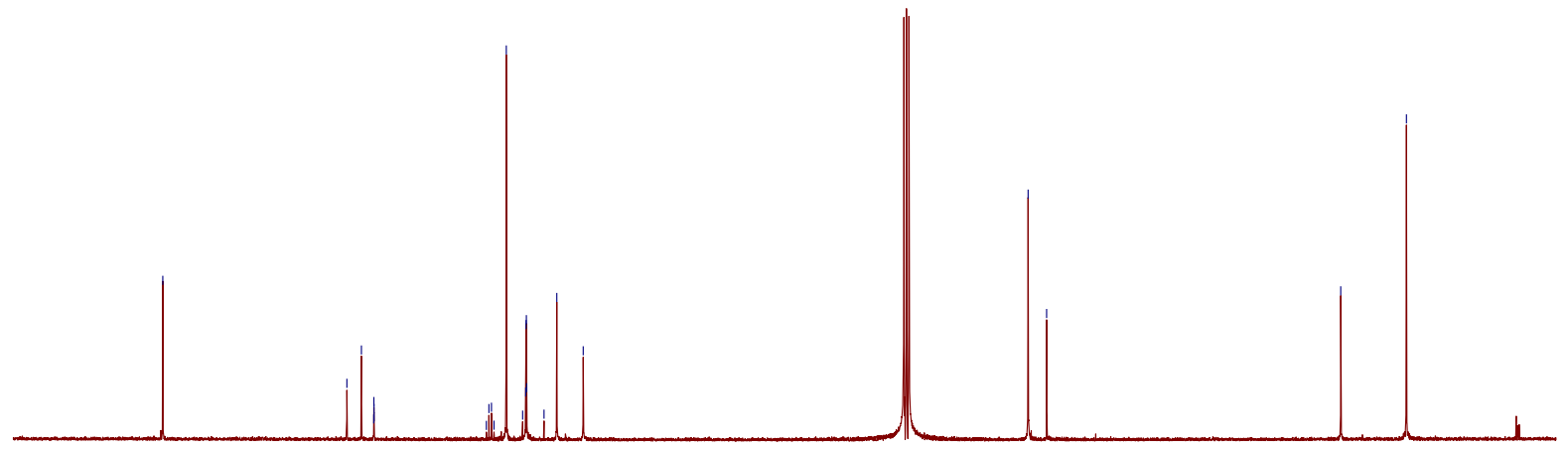

$\begin{array}{llllllllll}180 & 170 & 160 & 150 & 140 & 130 & 120 & 110 & 100 & 90\end{array}$

$\begin{array}{llll}80 & 70 & 60 & 50\end{array}$

$40 \quad 30$

$2010 \quad 0$ 


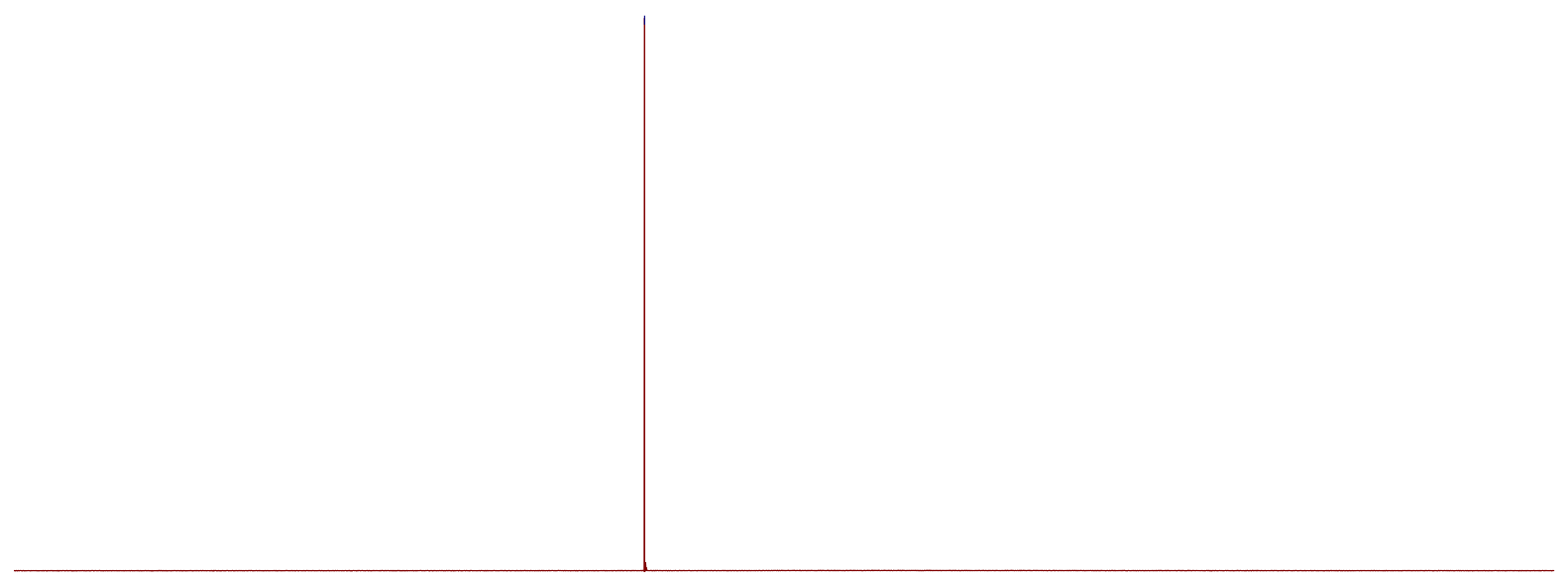

$\begin{array}{lllllllllllllllllllll}100 & 80 & 60 & 40 & 20 & 0 & -20 & -40 & -60 & -80 & -100 & -120 & -140 & -160 & -180 & -200 & -220 & -240 & -260 & -280 & -300\end{array}$

${ }^{1} \mathrm{H},{ }^{13} \mathrm{C}$ and ${ }^{19} \mathrm{~F}$ NMR spectra for product $3 \mathrm{ga}\left(400 \mathrm{MHz}, \mathrm{CDCl}_{3}\right)$ 


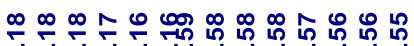
क्ष

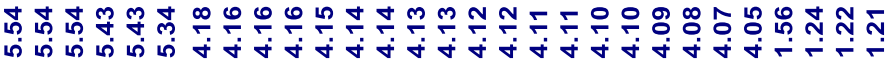<smiles>C=C(C(=C)C(C)(C(=O)OCC)C(=O)OCC)c1ccc([N+](=O)[O-])cc1</smiles>
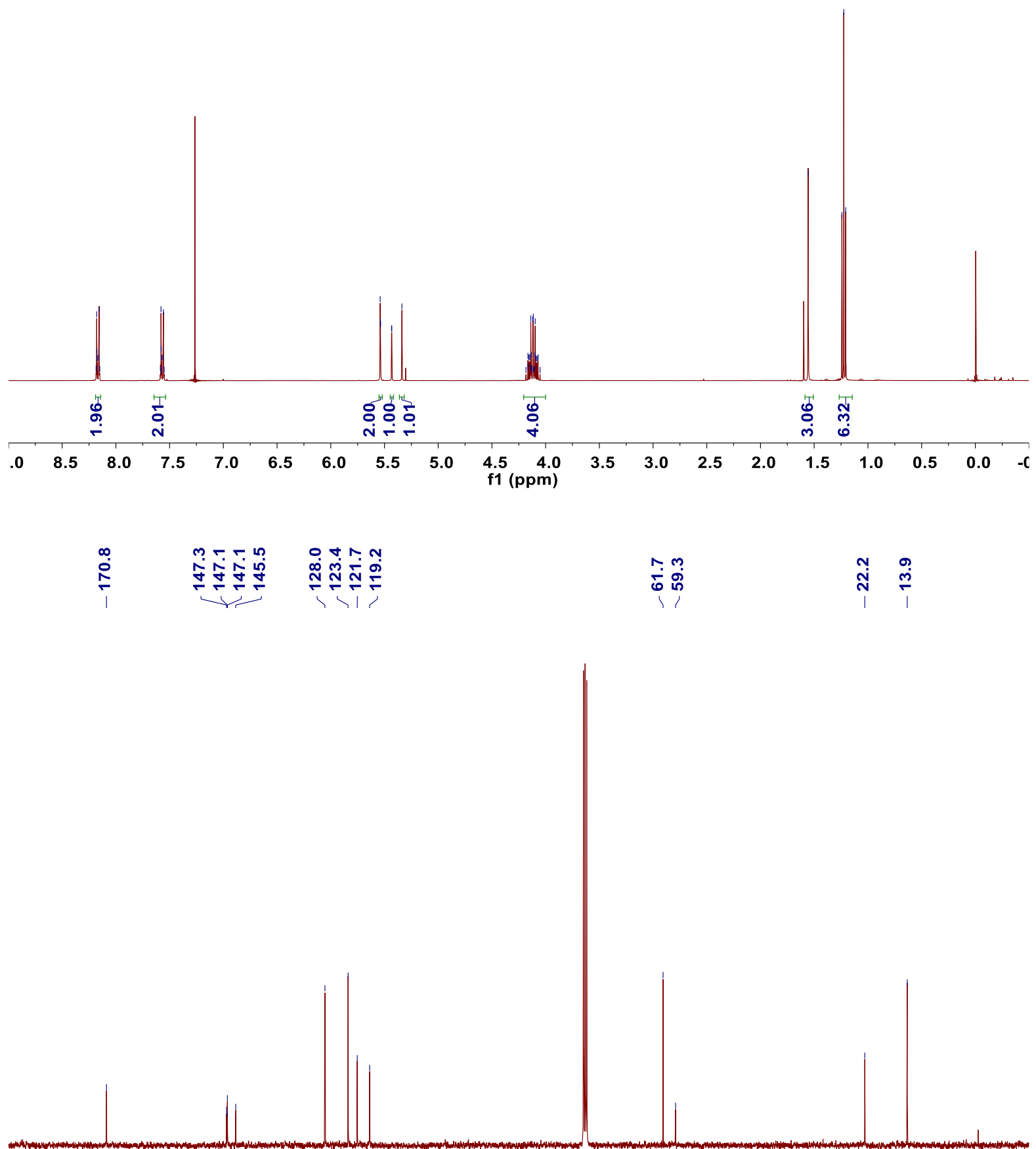

$\begin{array}{lllllllllll}90 & 180 & 170 & 160 & 150 & 140 & 130 & 120 & 110 & 100 \underset{\mathrm{f} 1}{90}(\mathrm{ppm}) & 80\end{array}$ $\begin{array}{llllllll}60 & 50 & 40 & 30 & 20 & 10 & 0 & -\end{array}$

${ }^{1} \mathrm{H}$ and ${ }^{13} \mathrm{C}$ NMR spectra for product 3 ha $\left(400 \mathrm{MHz}, \mathrm{CDCl}_{3}\right)$ 

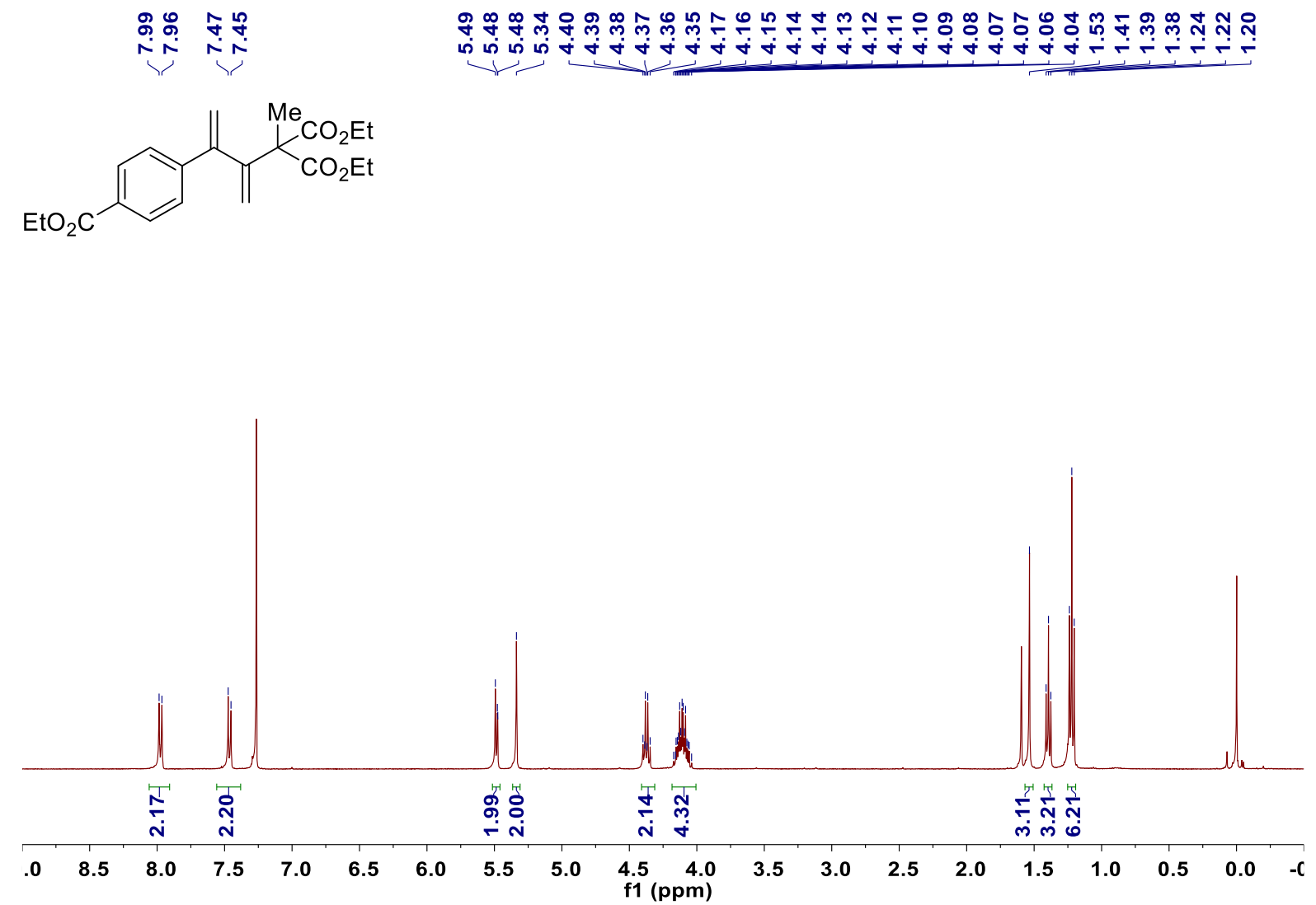

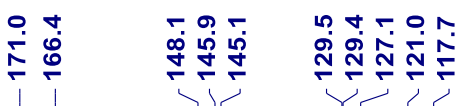

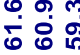

กั่

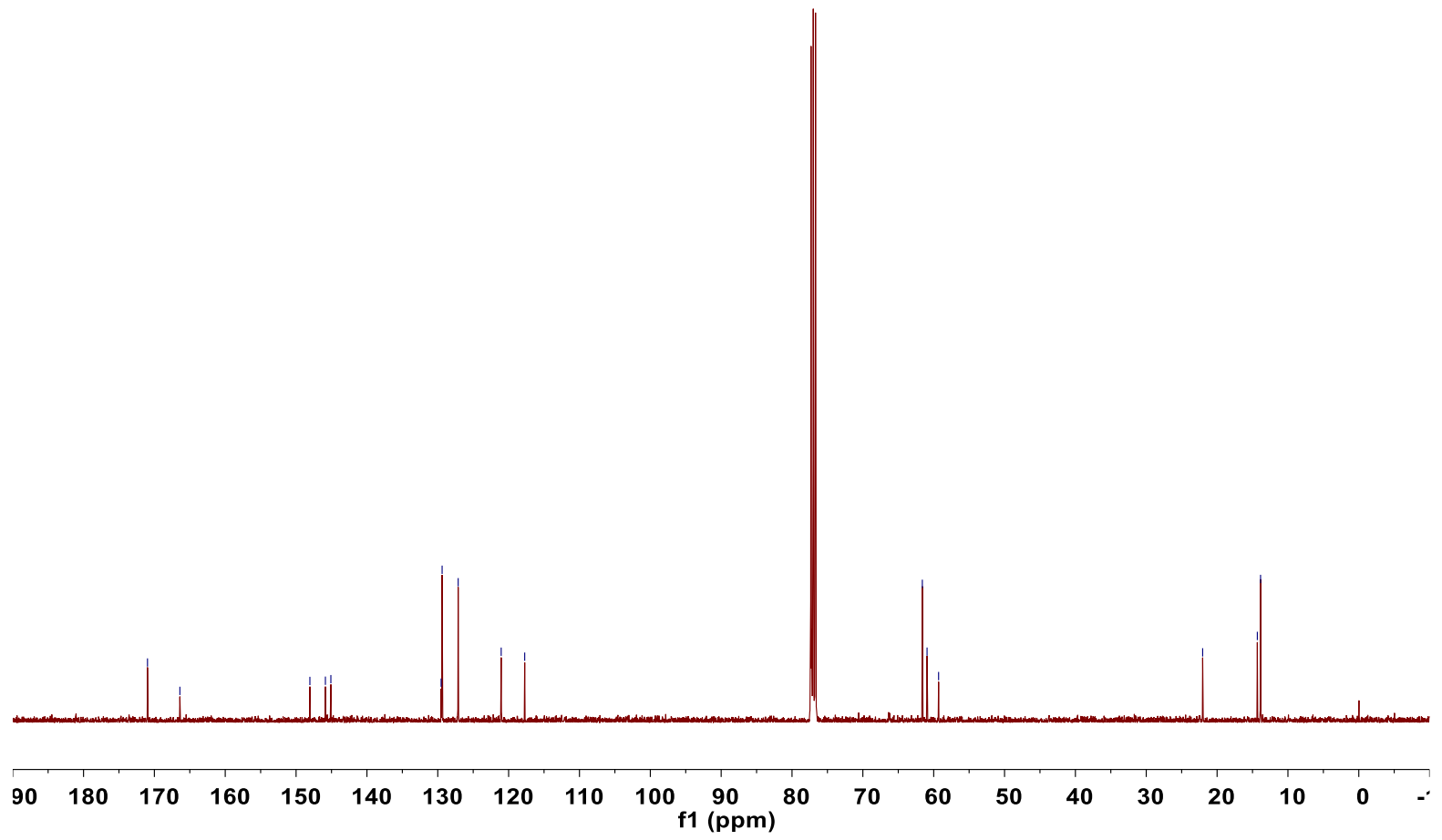

${ }^{1} \mathrm{H}$ and ${ }^{13} \mathrm{C}$ NMR spectra for product $3 i \mathrm{ia}\left(400 \mathrm{MHz}, \mathrm{CDCl}_{3}\right)$ 


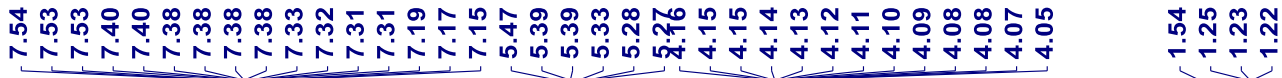

(I)

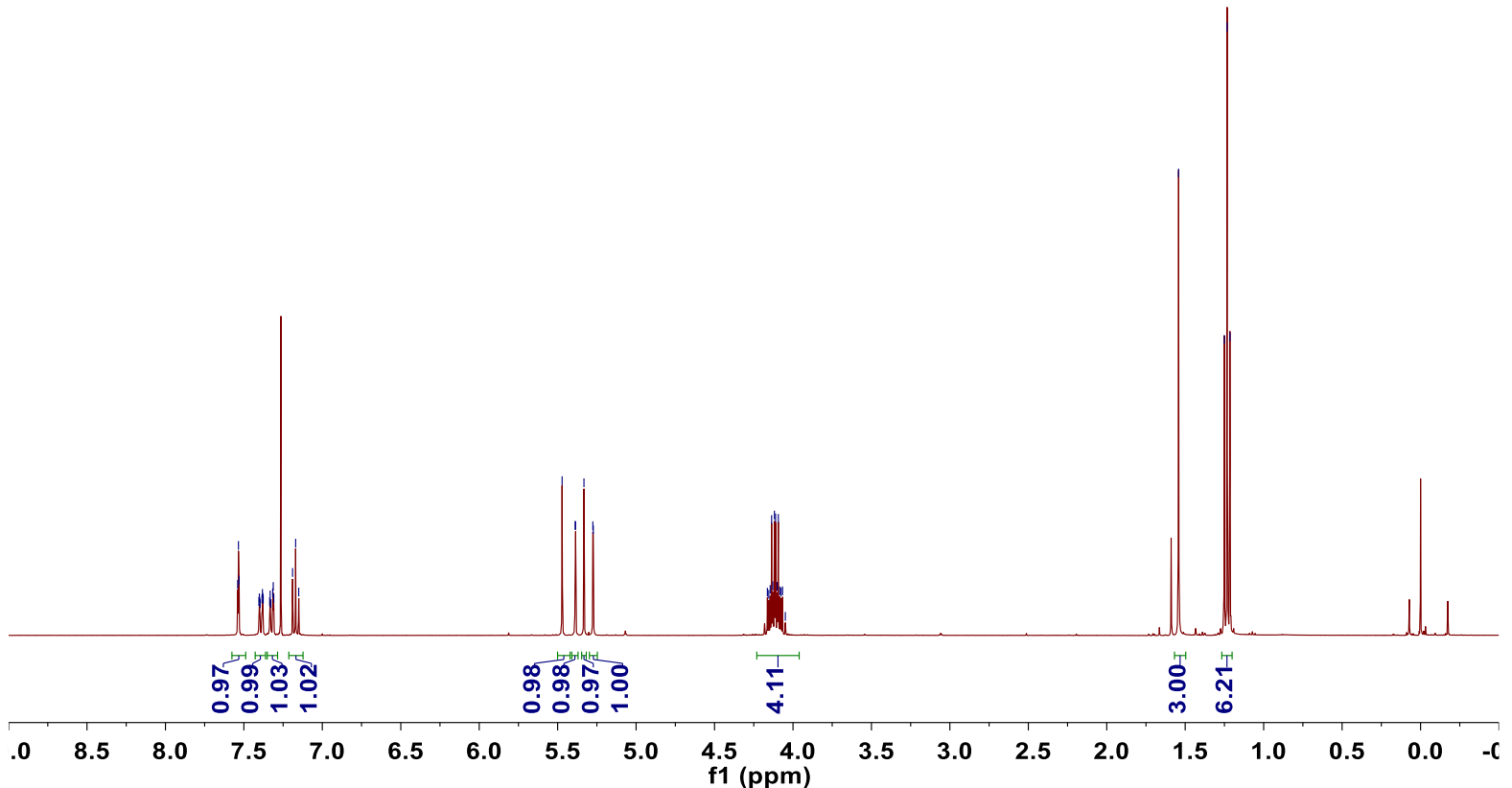

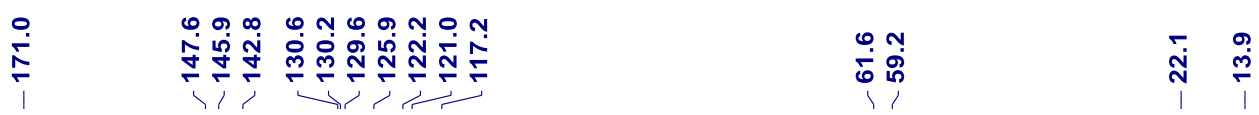

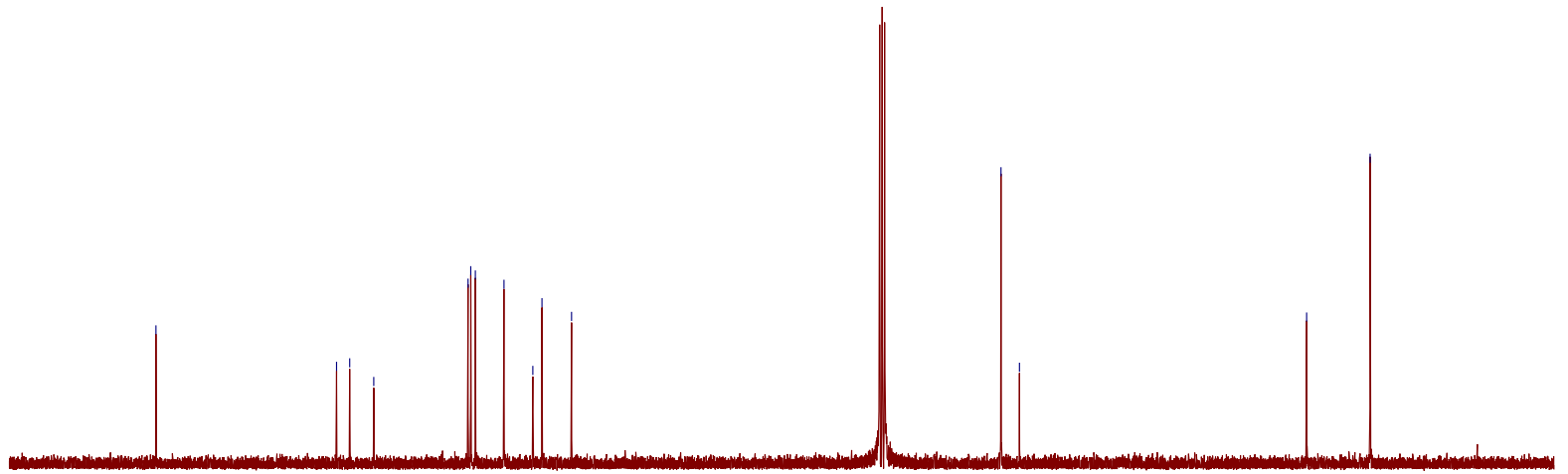

$\begin{array}{llllllllllllllllllllll}90 & 180 & 170 & 160 & 150 & 140 & 130 & 120 & 110 & 100 & 90 & 80 & 70 & 60 & 50 & 40 & 30 & 20 & 10 & 0 & -\end{array}$

${ }^{1} \mathrm{H}$ and ${ }^{13} \mathrm{C}$ NMR spectra for product $3 \mathrm{ja}\left(400 \mathrm{MHz}, \mathrm{CDCl}_{3}\right)$ 


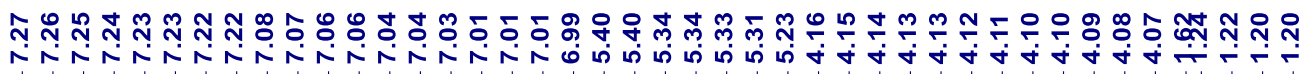<smiles>C=C(C(=C)C(C)(OCC)C(C)=O)c1ccccc1F</smiles>

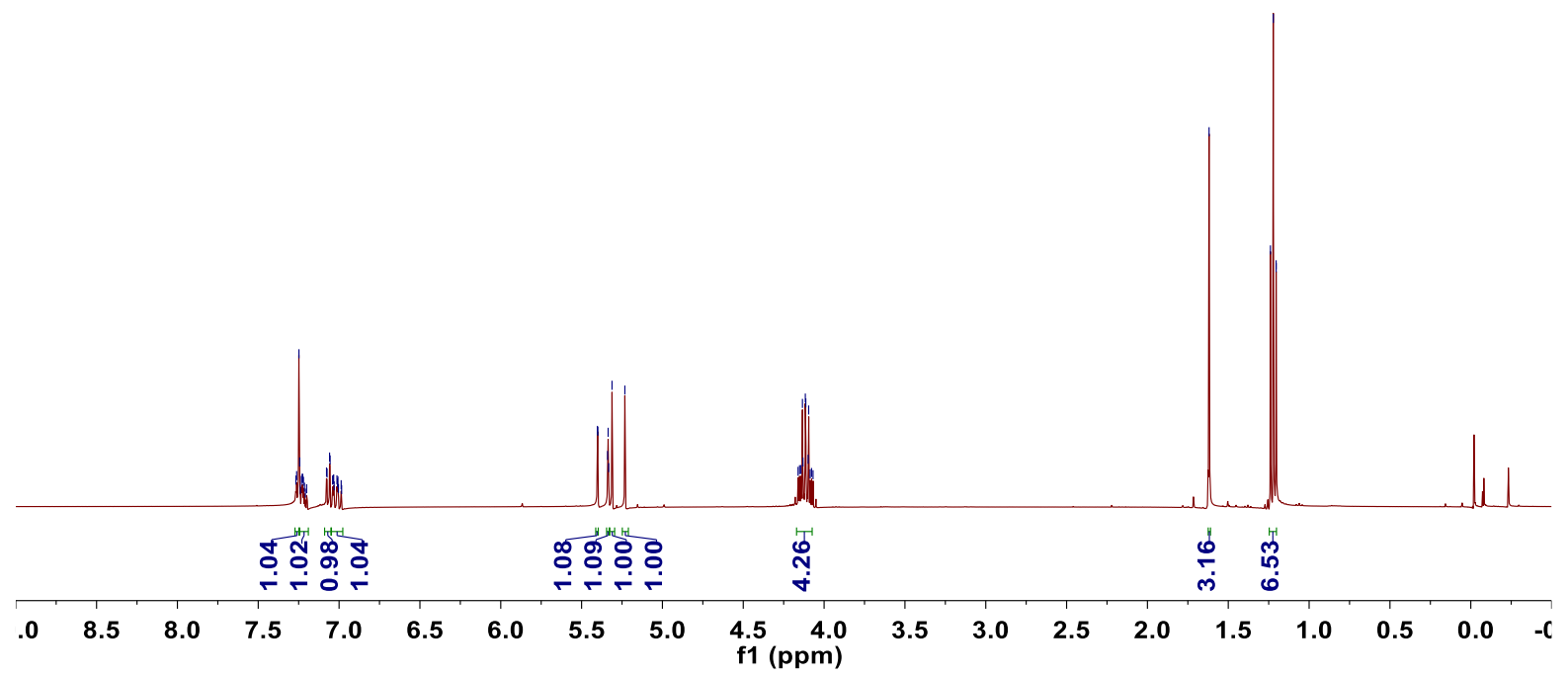

†

फ़்

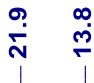

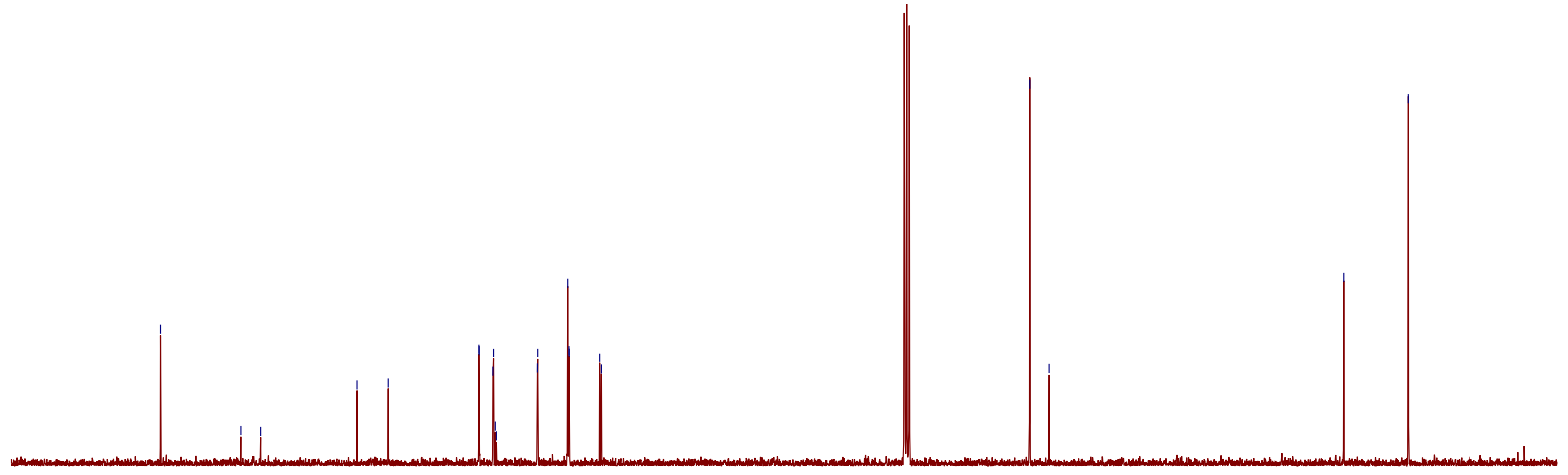

$\begin{array}{lllllllllll}90 & 180 & 170 & 160 & 150 & 140 & 130 & 120 & 110 & 100 & 90 \\ 91\end{array}$

8070

6050

$40 \quad 30$

$2010 \quad 0$ 


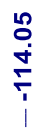

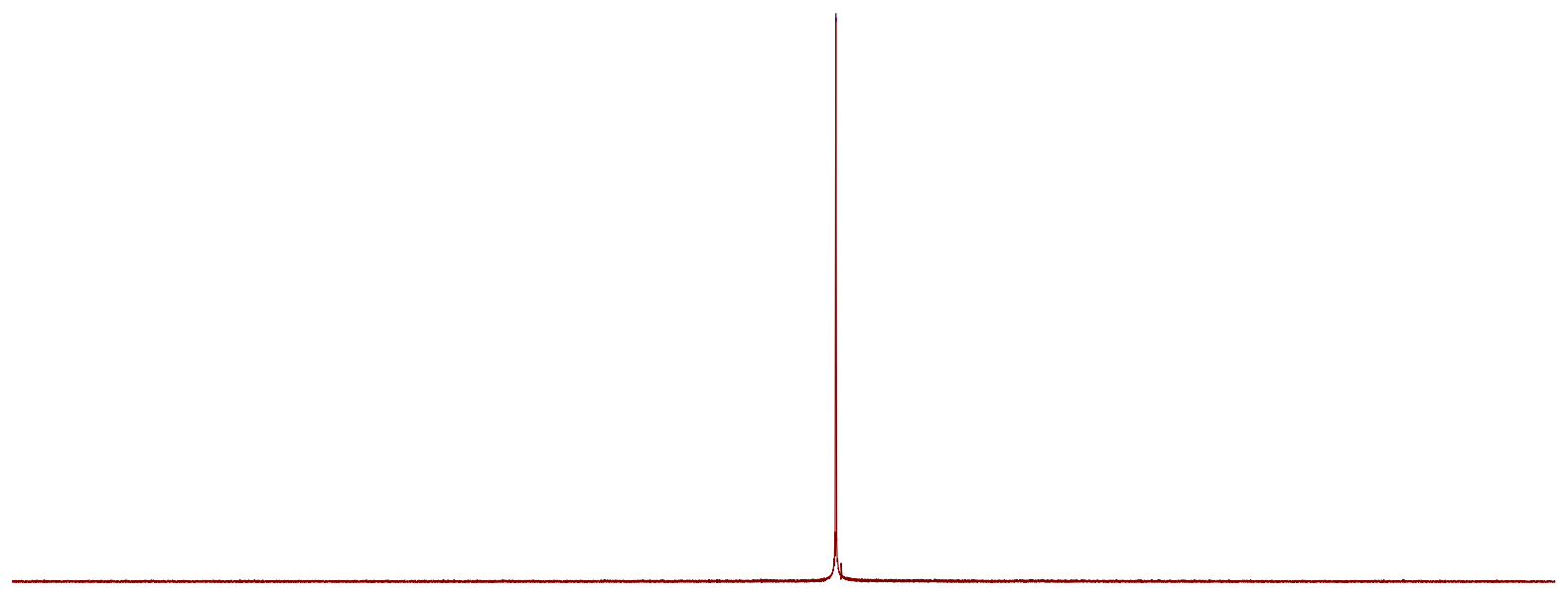
$\begin{array}{lllllllllllllllllllll}100 & 80 & 60 & 40 & 20 & 0 & -20 & -40 & -60 & -80 & -100 & -120 & -140 & -160 & -180 & -200 & -220 & -240 & -260 & -280 & -300\end{array}$ ${ }^{1} \mathrm{H},{ }^{13} \mathrm{C}$ and ${ }^{19} \mathrm{~F}$ NMR spectra for product $3 \mathrm{ka}\left(400 \mathrm{MHz}, \mathrm{CDCl}_{3}\right)$ 
穴둔<smiles>C=C(C(=C)C(C)(C)OCC)c1ccccc1C</smiles>
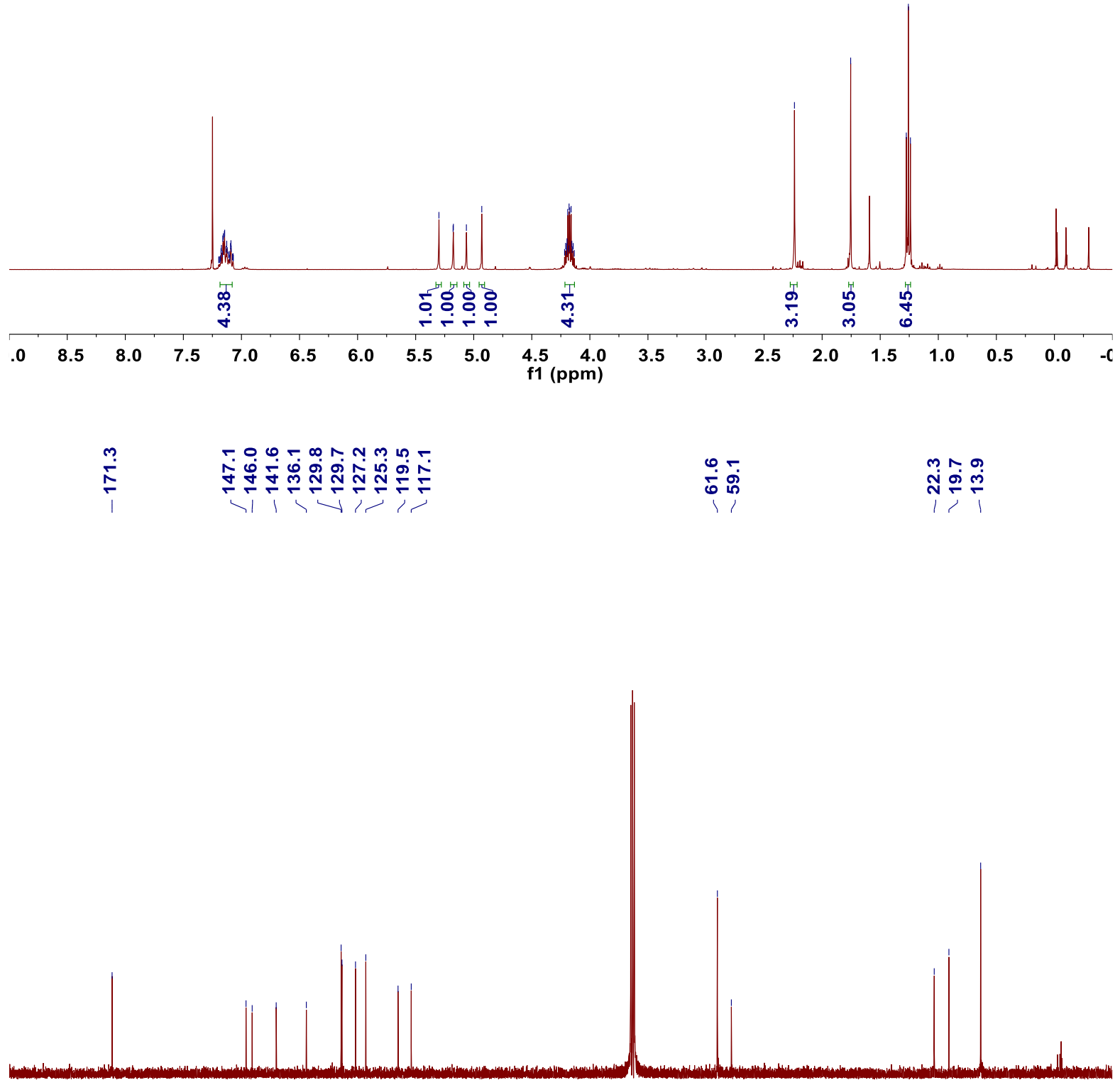

$\begin{array}{llllllllll}180 & 170 & 160 & 150 & 140 & 130 & 120 & 110 & 100 & 90 \\ 90\end{array}$ 6050 $40 \quad 30$ $2010 \quad 0 \quad-$

${ }^{1} \mathrm{H}$ and ${ }^{13} \mathrm{C}$ NMR spectra for product 3la (400 $\left.\mathrm{MHz}, \mathrm{CDCl}_{3}\right)$ 


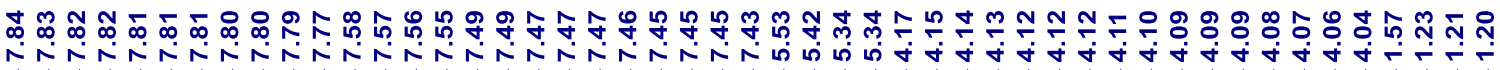<smiles>C=C(C(=C)C(C)(C(=O)OCC)C(=O)OCC)c1ccc2ccccc2c1</smiles>

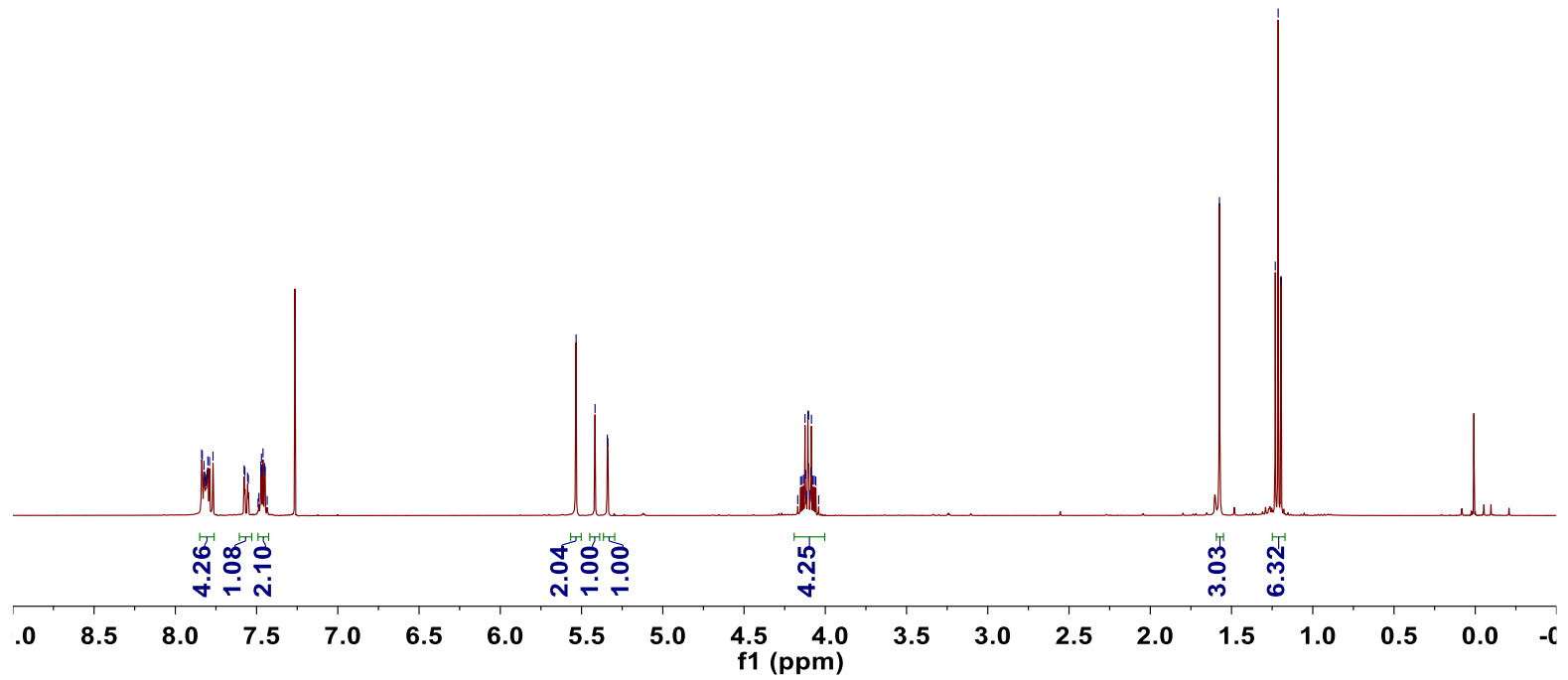

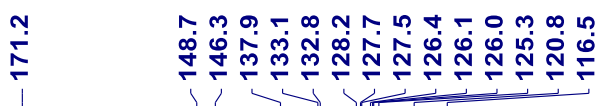

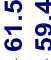

$\stackrel{\circ}{\text { N }} \stackrel{\infty}{\stackrel{m}{\Gamma}}$

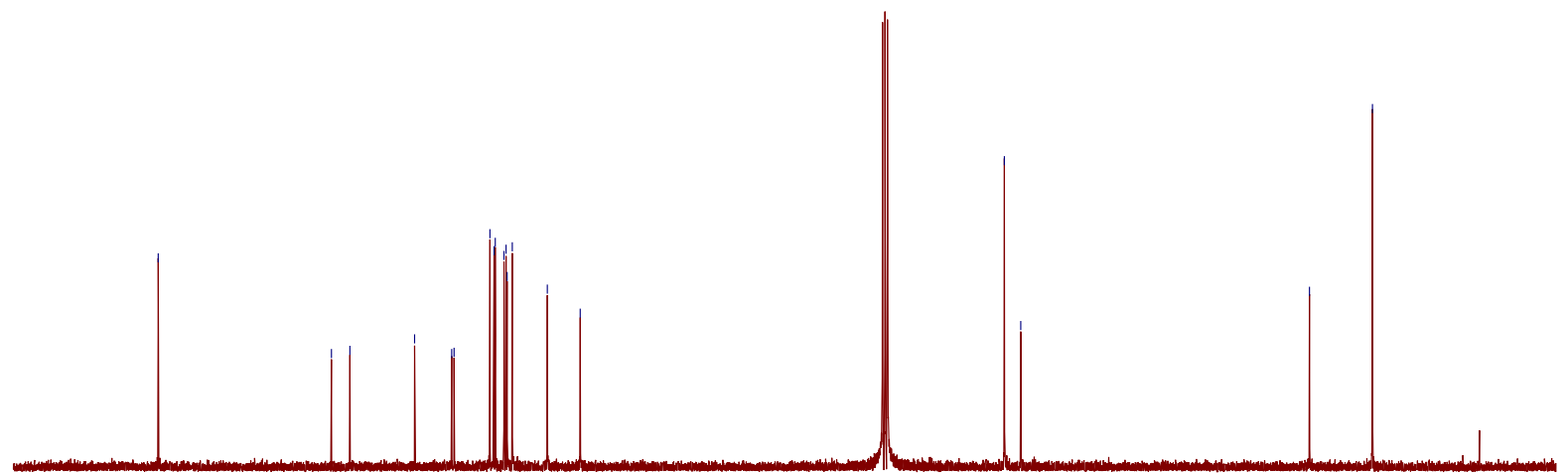

$\begin{array}{llllllllllllllllllllll}180 & 170 & 160 & 150 & 140 & 130 & 120 & 110 & 100 & 90 & 80 & 70 & 60 & 50 & 40 & 30 & 20 & 10 & 0 & -\end{array}$

${ }^{1} \mathrm{H}$ and ${ }^{13} \mathrm{C}$ NMR spectra for product $3 \mathrm{ma}\left(400 \mathrm{MHz}, \mathrm{CDCl}_{3}\right)$ 

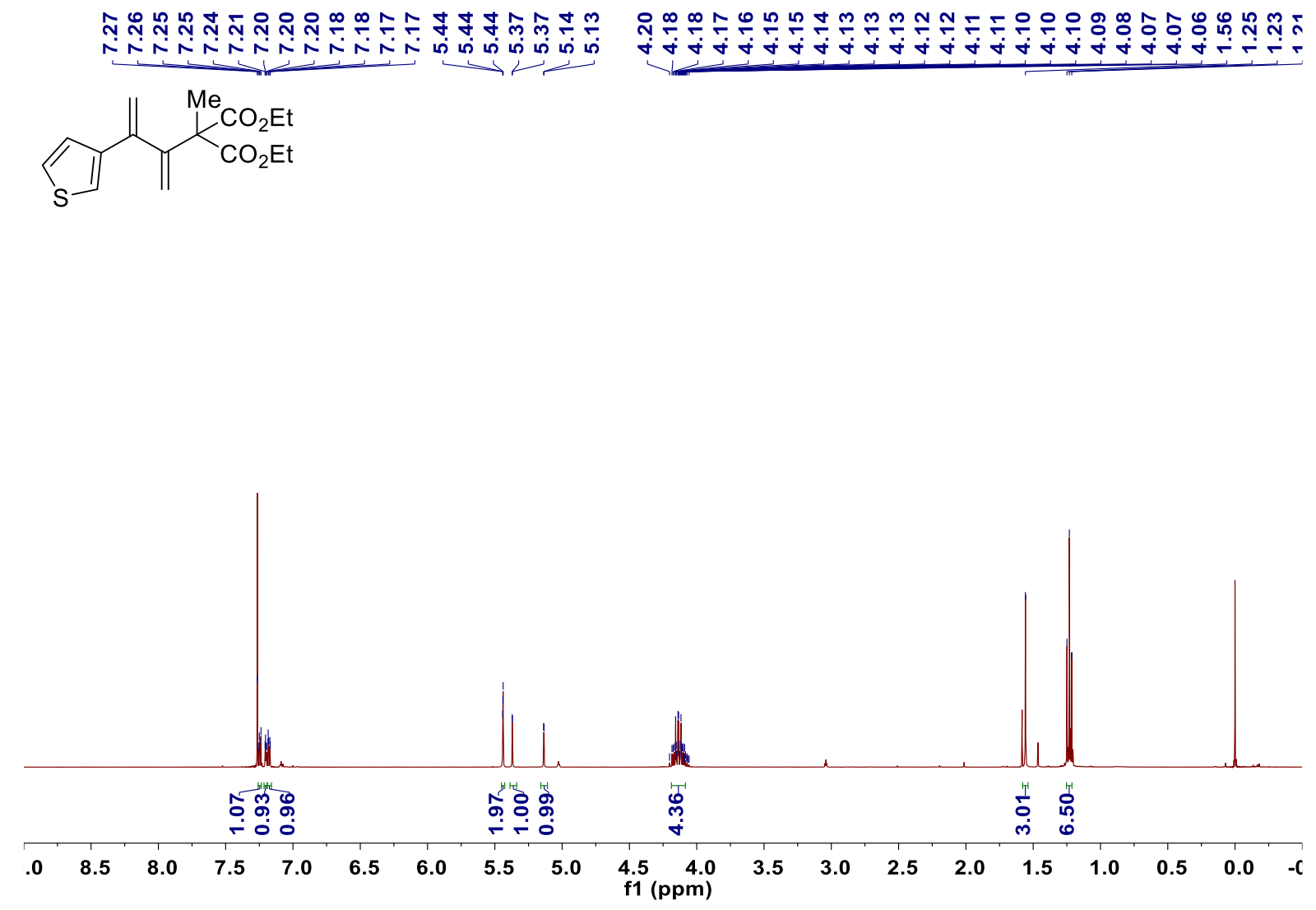

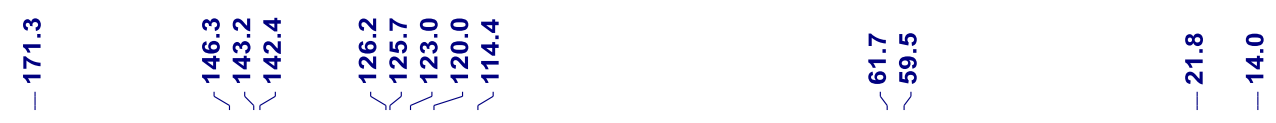

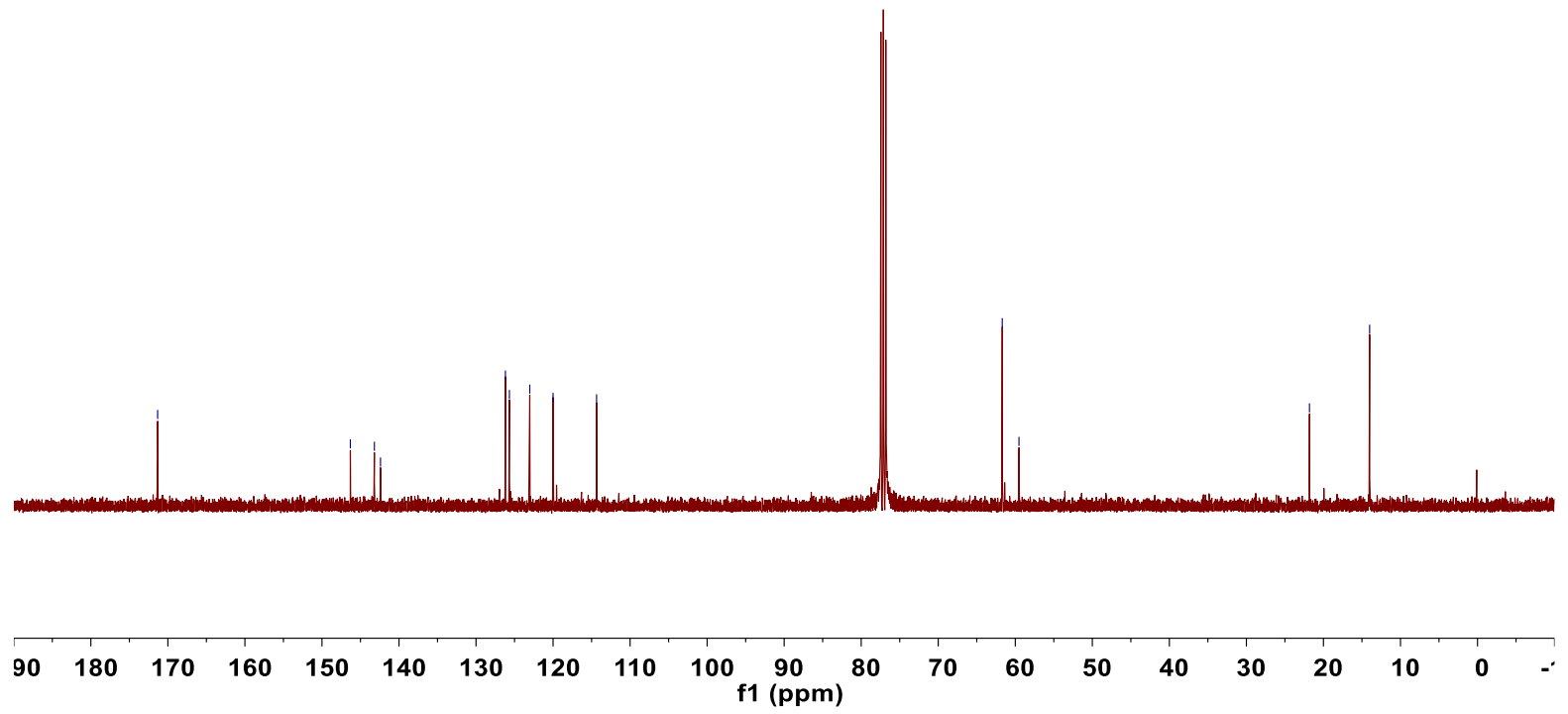

${ }^{1} \mathrm{H}$ and ${ }^{13} \mathrm{C}$ NMR spectra for product $3 \mathrm{na}\left(400 \mathrm{MHz}, \mathrm{CDCl}_{3}\right)$ 


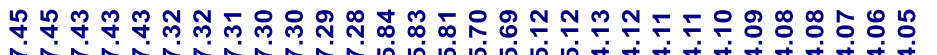

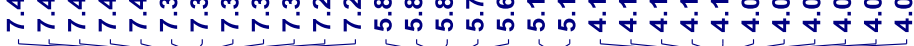<smiles>C=C(C(=O)OCC)/C(=C\C(=O)c1ccccc1)C(C)(C)OCC</smiles>

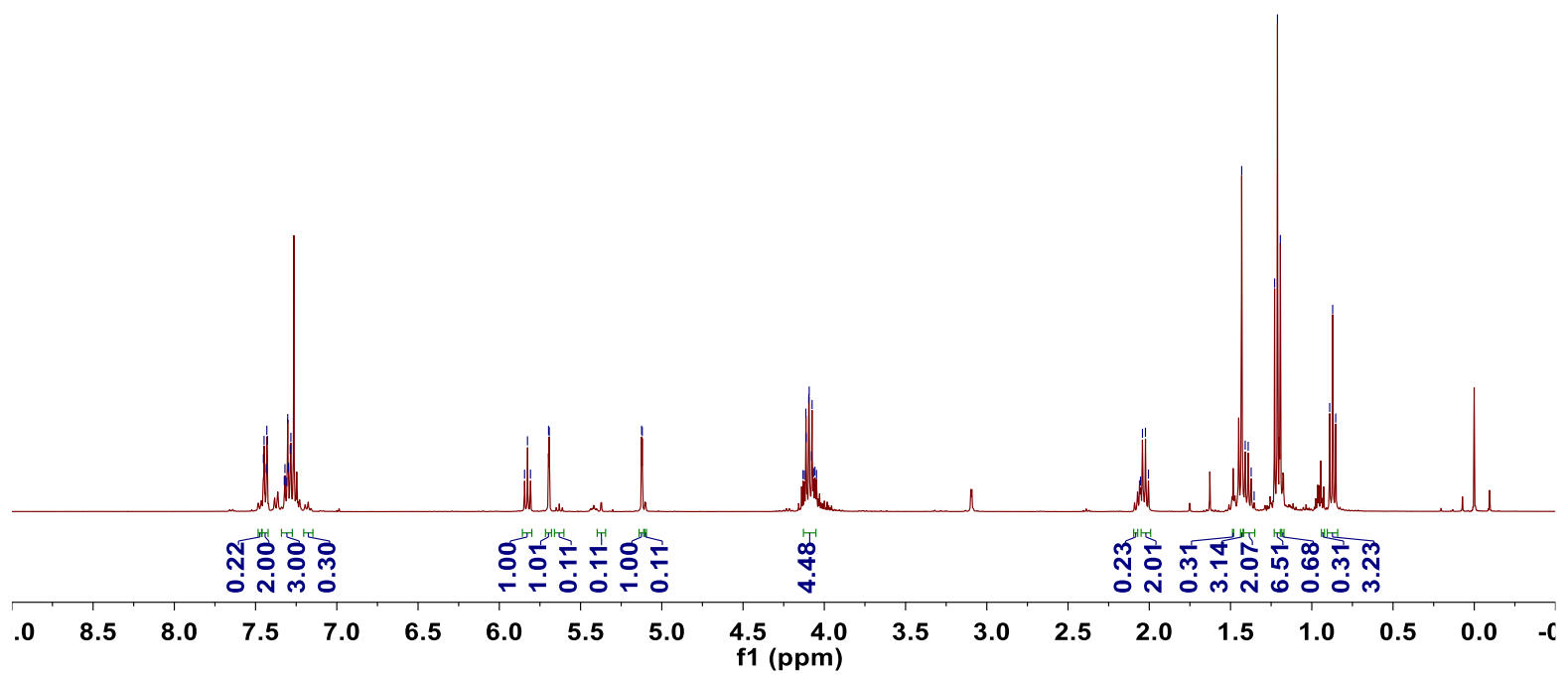

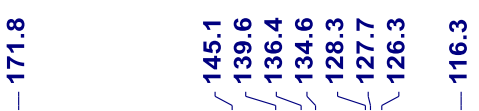

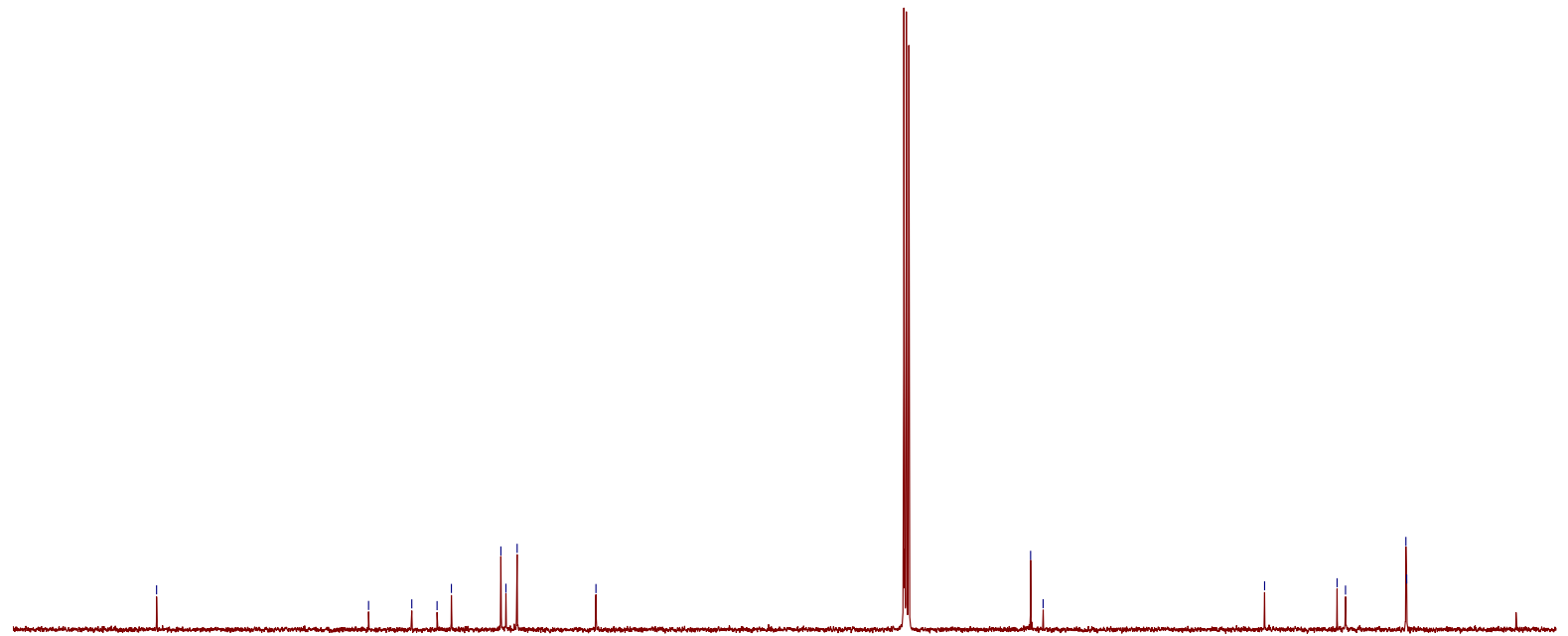

$\begin{array}{llllllllll}180 & 170 & 160 & 150 & 140 & 130 & 120 & 110 & 100 & 90 \\ 91 & & \end{array}$

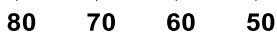

$2010 \quad 0$

${ }^{1} \mathrm{H}$ and ${ }^{13} \mathrm{C}$ NMR a spectra for product $30 a\left(400 \mathrm{MHz}, \mathrm{CDCl}_{3}\right)$ 

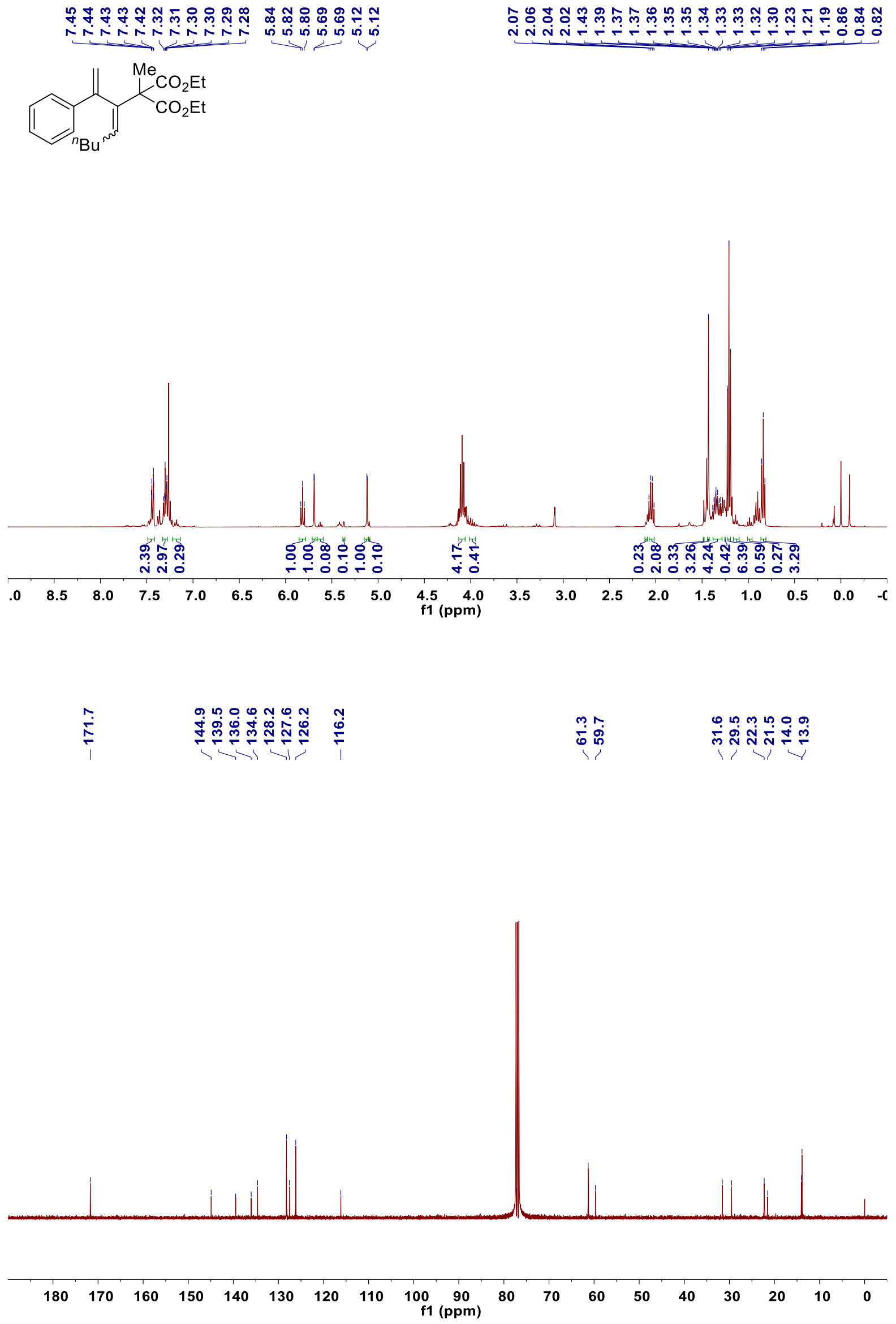


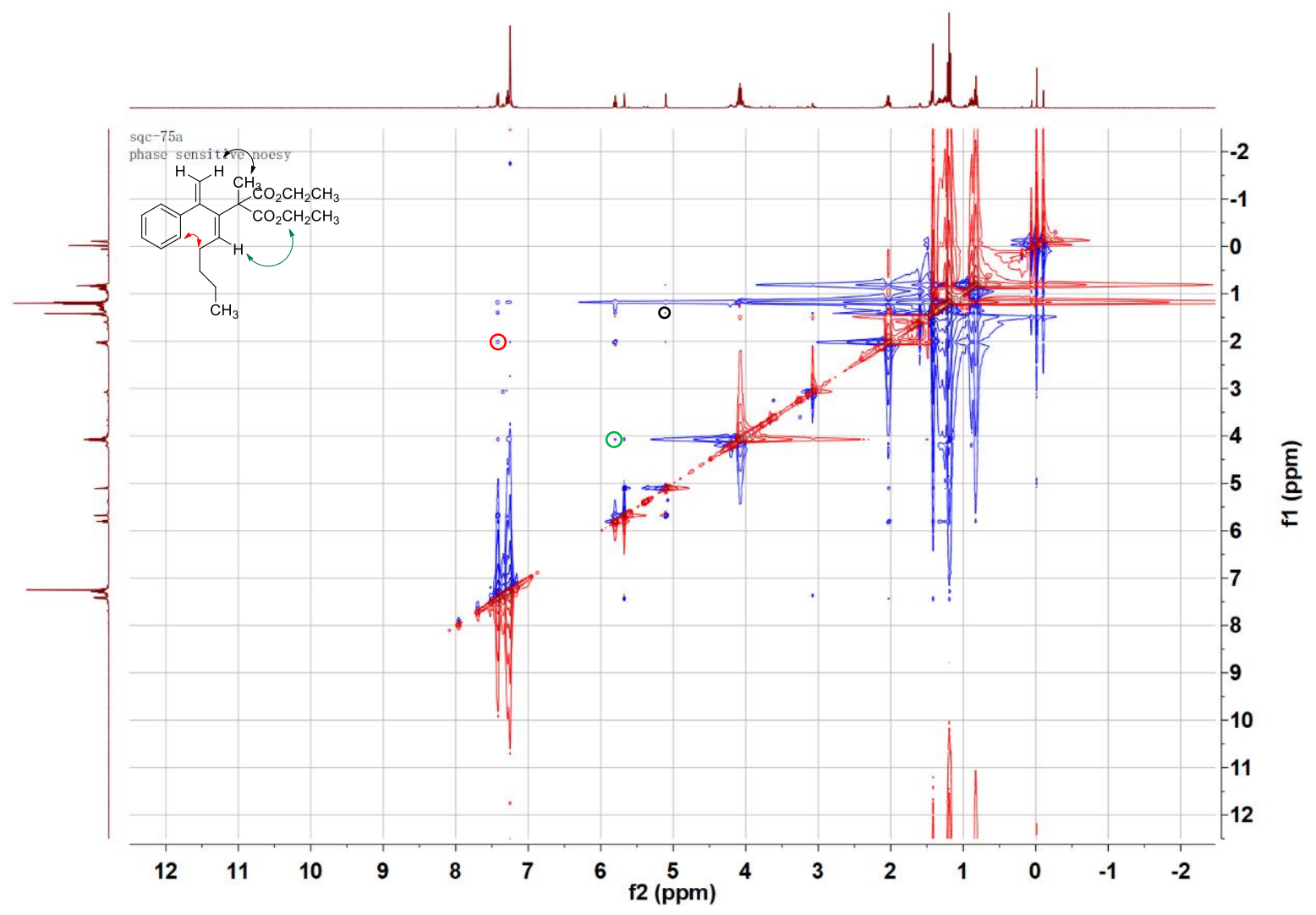

${ }^{1} \mathrm{H},{ }^{13} \mathrm{C}$ NMR and NOESY spectra for product $3 p a\left(400 \mathrm{MHz}, \mathrm{CDCl}_{3}\right)$ 


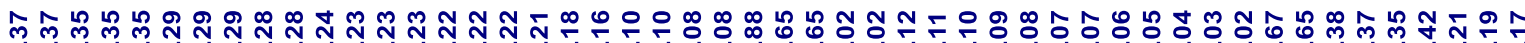

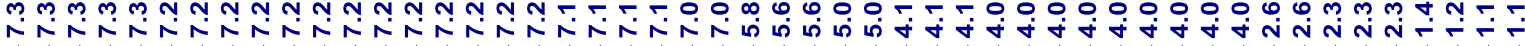<smiles>C=C(C(=CCCc1ccccc1)C(C)(OCC)OCC)c1ccccc1</smiles>

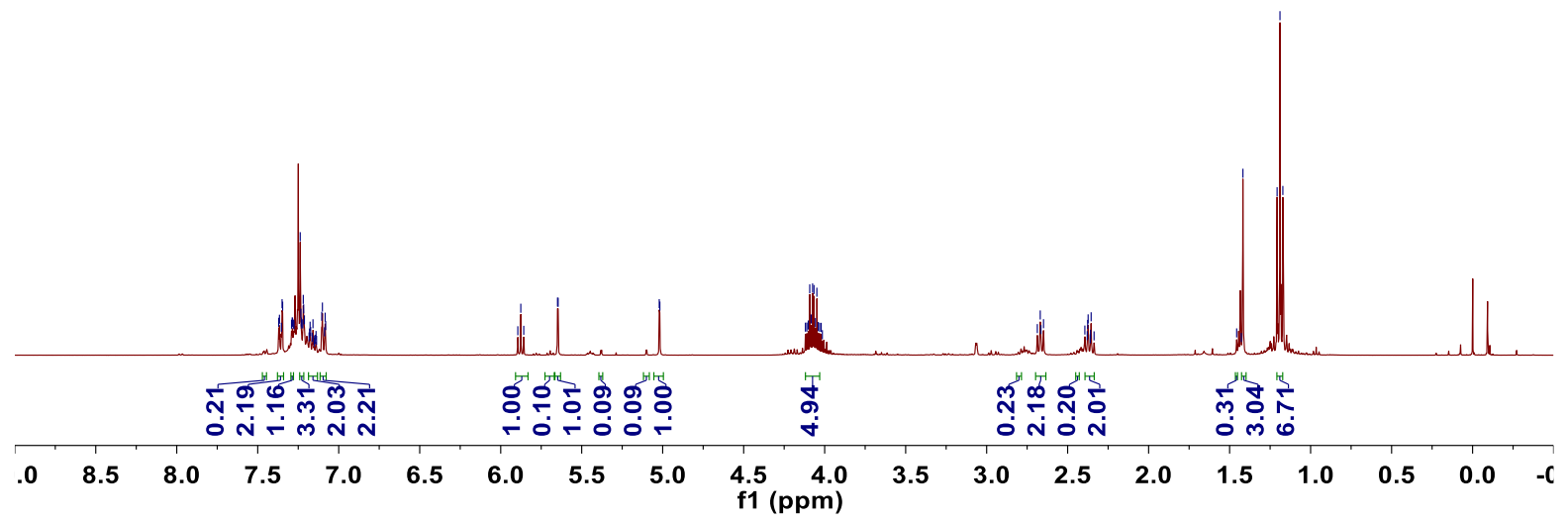

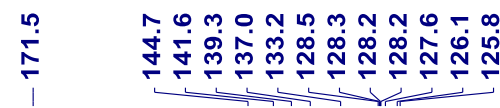

mị

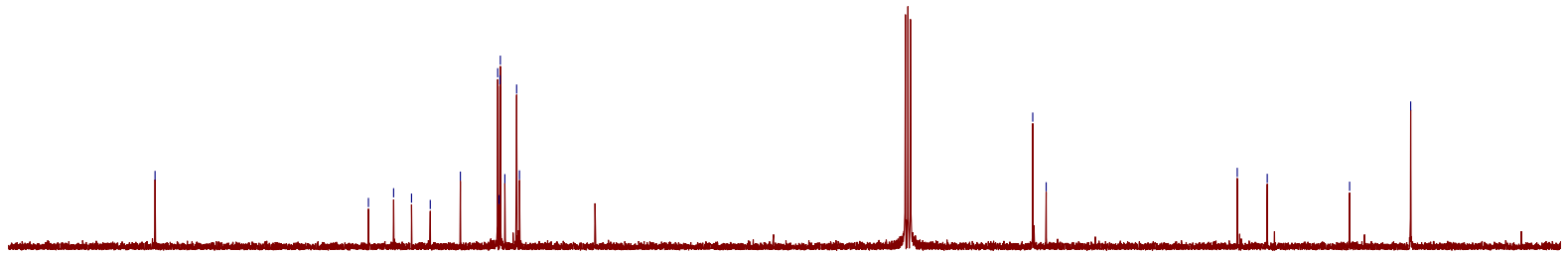

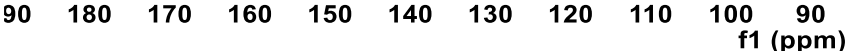

${ }^{1} \mathrm{H}$ and ${ }^{13} \mathrm{C}$ NMR spectra for product $3 q a\left(400 \mathrm{MHz}, \mathrm{CDCl}_{3}\right)$ 
చิ

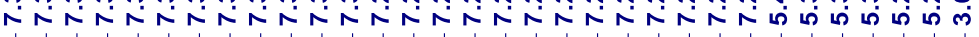

$\overbrace{\mathrm{CO}_{2} \mathrm{Me}}^{\mathrm{Me}}$

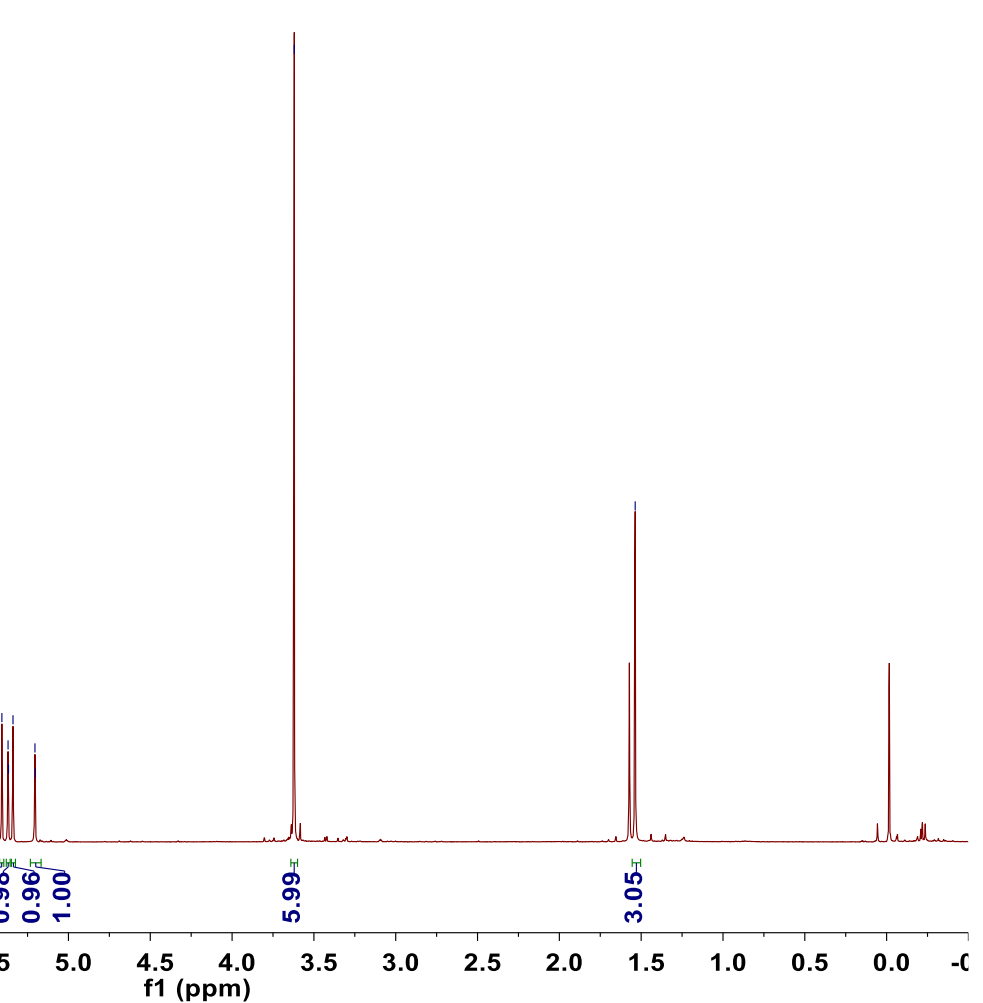

萬

$\stackrel{?}{\stackrel{\leftrightarrow}{N}}$

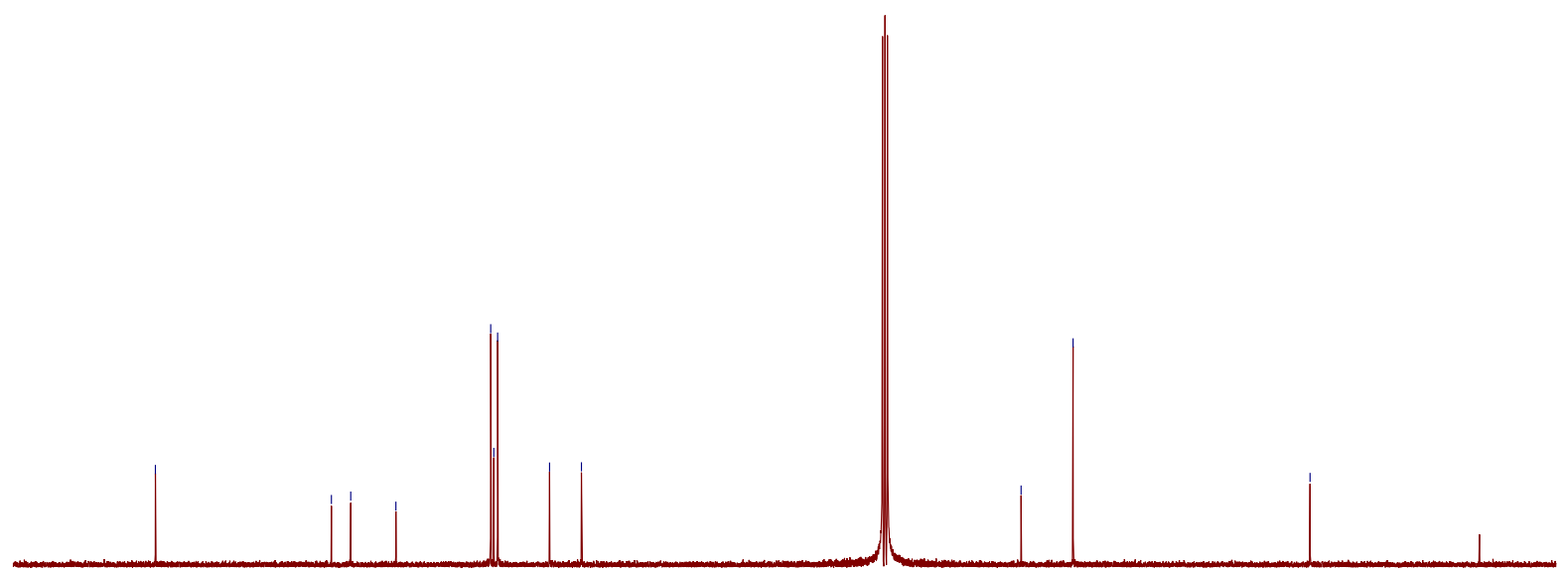

$90 \quad 180 \quad 170 \quad 160 \quad 150 \quad 140 \quad 130 \quad 120 \quad 110 \quad 100 \underset{\mathrm{f} 1(\mathrm{ppm})}{90} 80$

6050

(ppm)

${ }^{1} \mathrm{H}$ and ${ }^{13} \mathrm{C}$ NMR spectra for product $3 \mathrm{ab}\left(400 \mathrm{MHz}, \mathrm{CDCl}_{3}\right)$ 


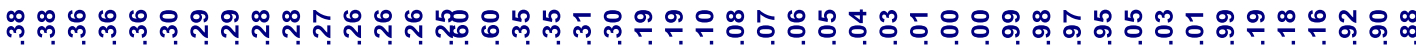

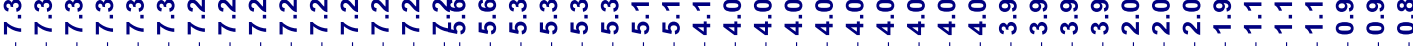<smiles>C=C(C(=C)C(CC)(COCC)C(=O)OCC)c1ccccc1</smiles>

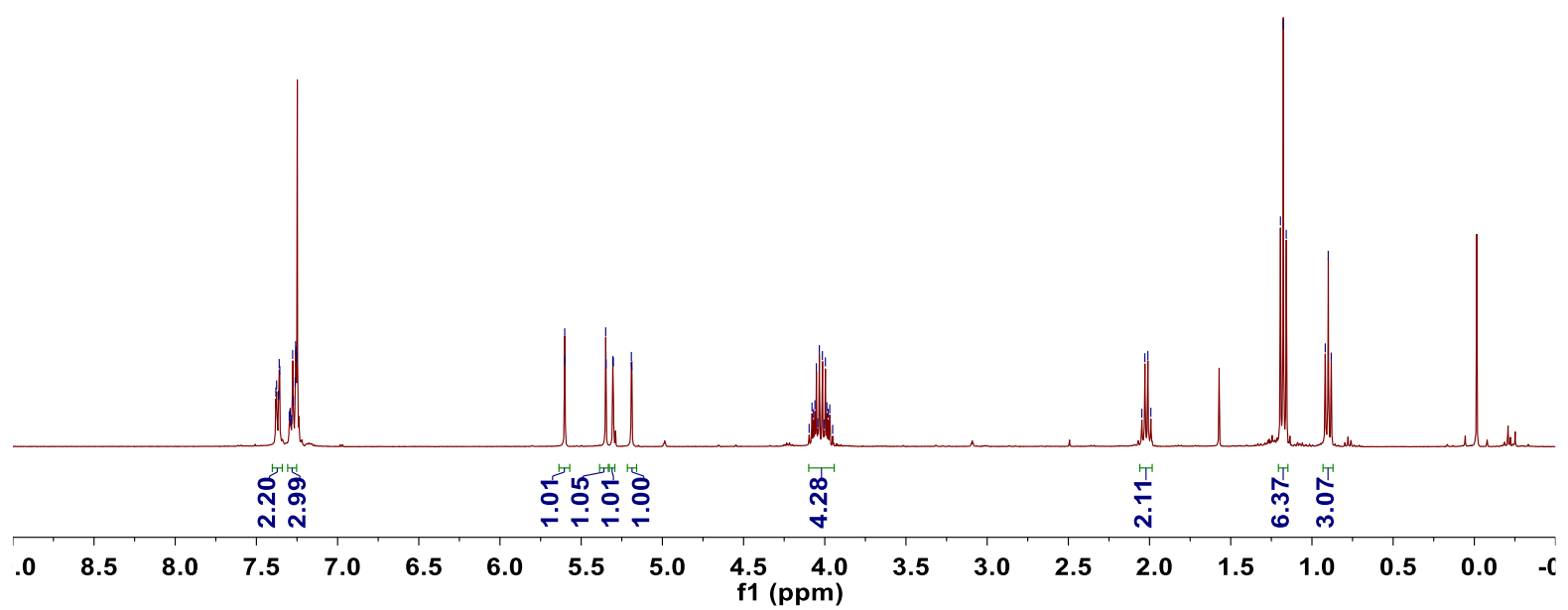

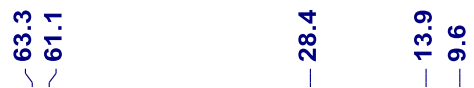

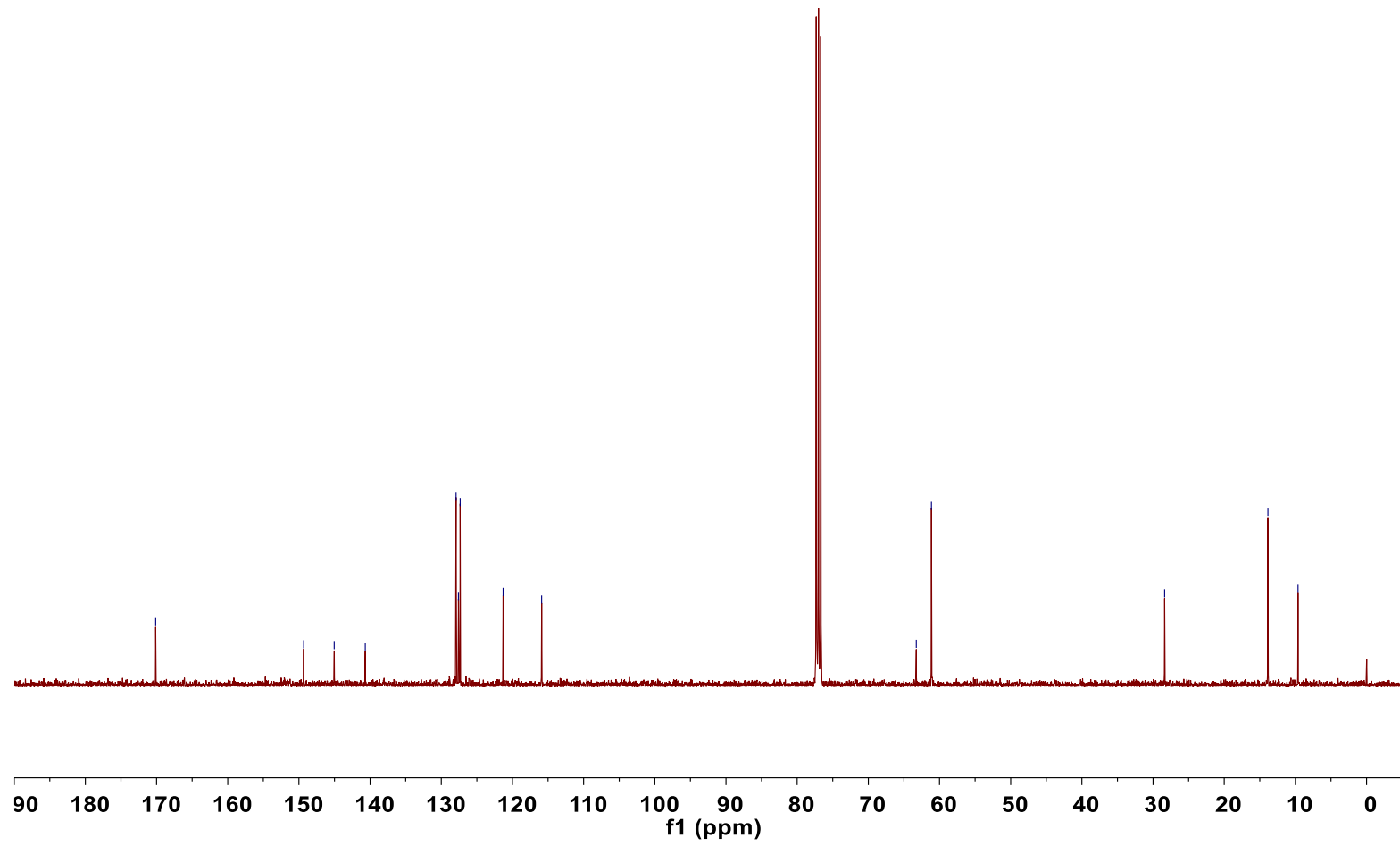

${ }^{1} \mathrm{H}$ and ${ }^{13} \mathrm{C}$ NMR spectra for product $3 \mathrm{ac}\left(400 \mathrm{MHz}, \mathrm{CDCl}_{3}\right)$ 


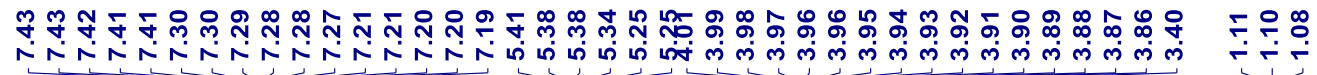<smiles>C=C(C(=C)C(Cc1ccccc1)(C(=O)OCC)C(=O)OCC)c1ccccc1</smiles>

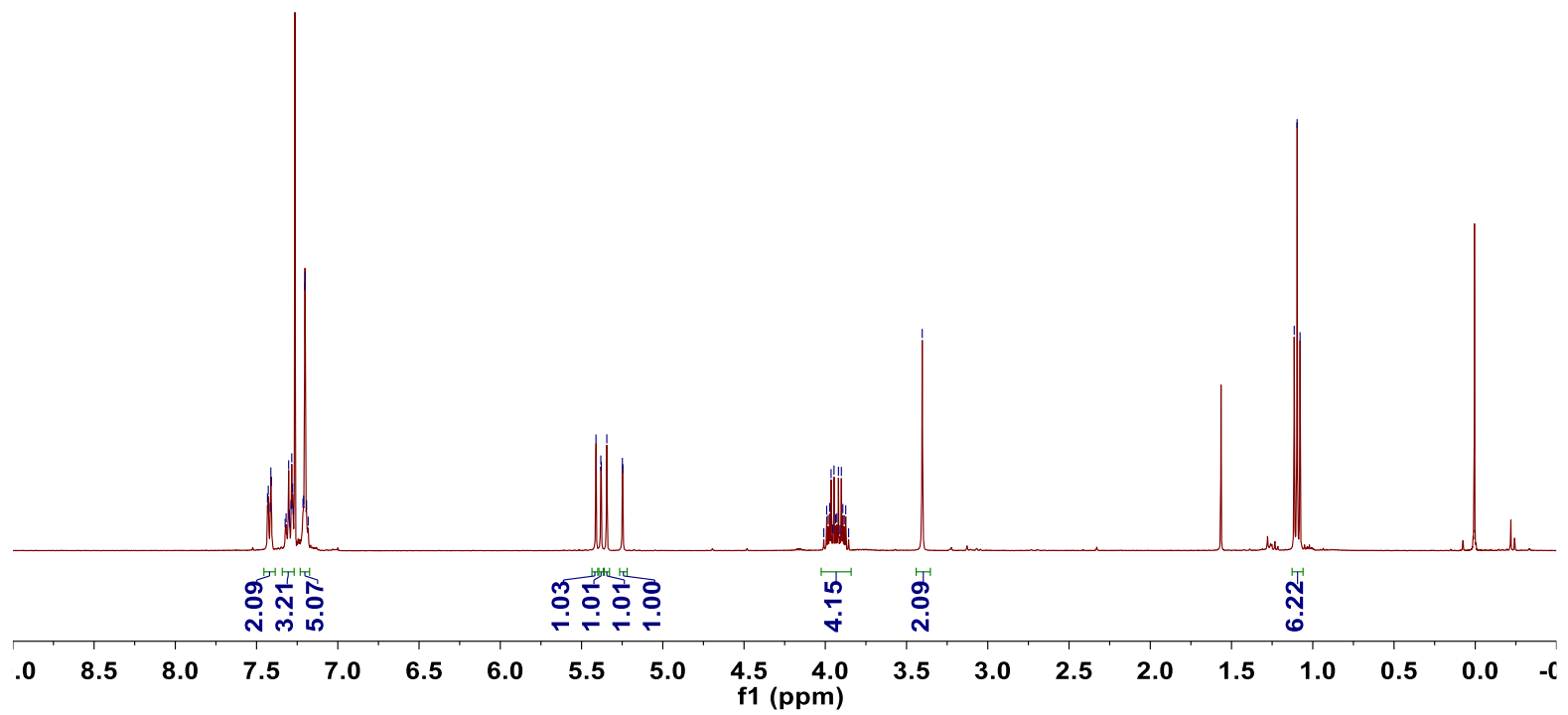

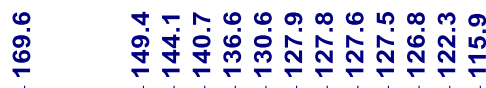

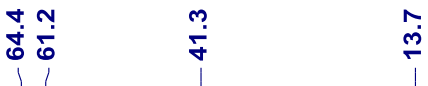

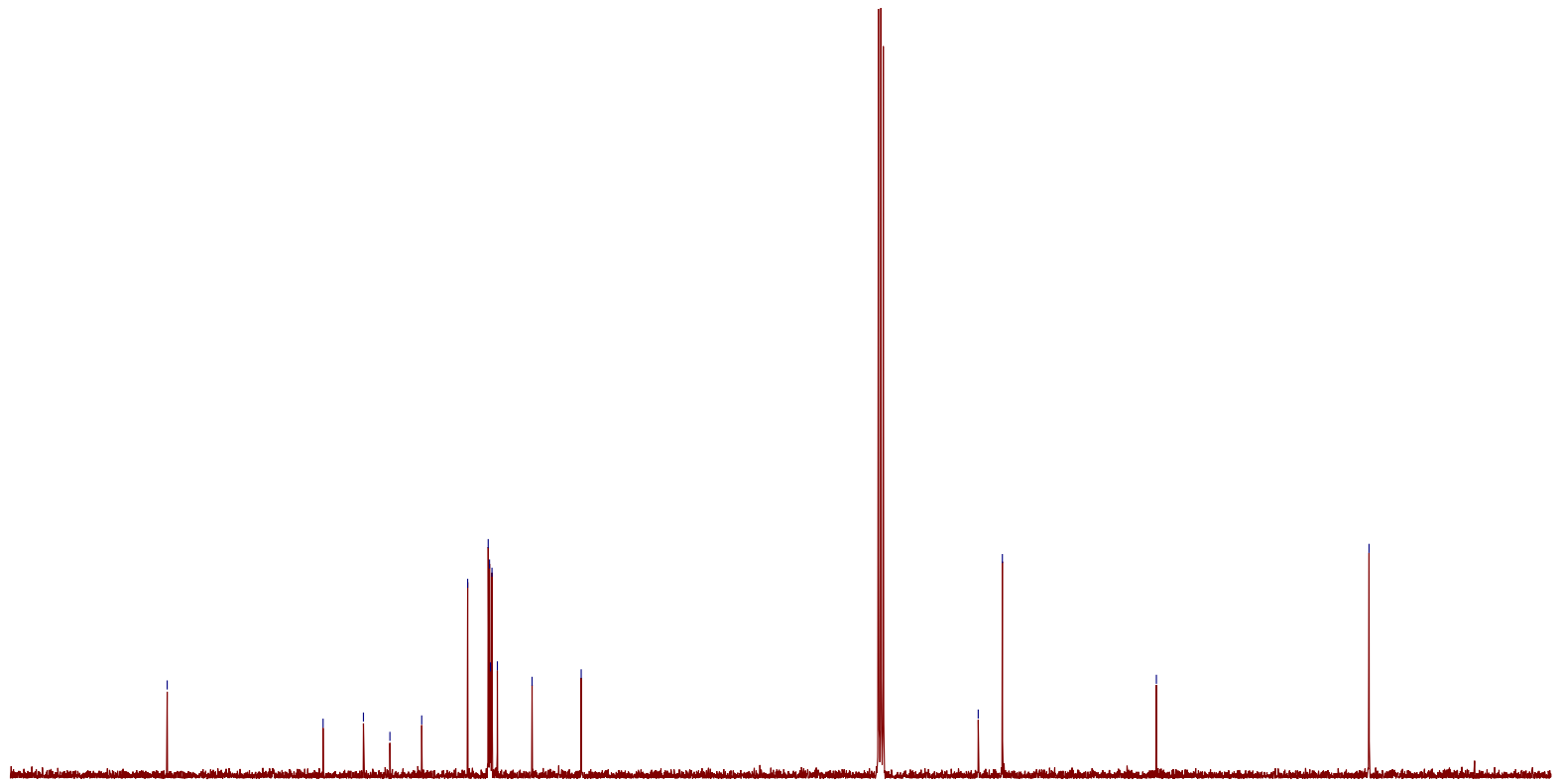

$\begin{array}{lllllllllllllllllllllll}90 & 180 & 170 & 160 & 150 & 140 & 130 & 120 & 110 & 100 & 90 & 80 & 70 & 60 & 50 & 40 & 30 & 20 & 10 & 0 & -\end{array}$

${ }^{1} \mathrm{H}$ and ${ }^{13} \mathrm{C}$ NMR spectra for product $3 \mathrm{ad}\left(400 \mathrm{MHz}, \mathrm{CDCl}_{3}\right)$ 


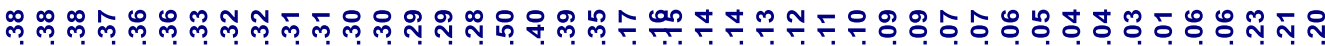

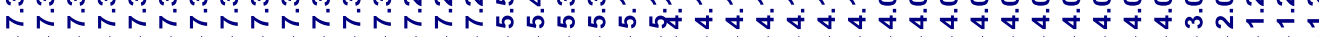

$\overbrace{\mathrm{CO}_{2} \mathrm{Et}}^{\mathrm{Cc}}$

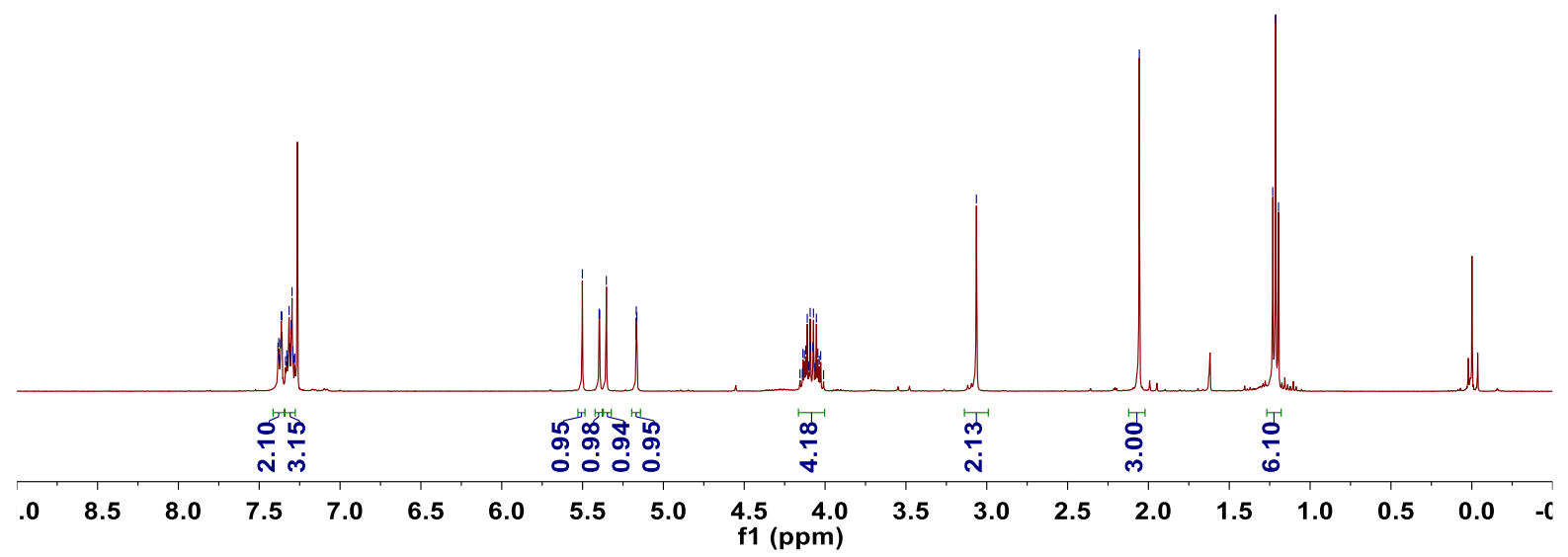

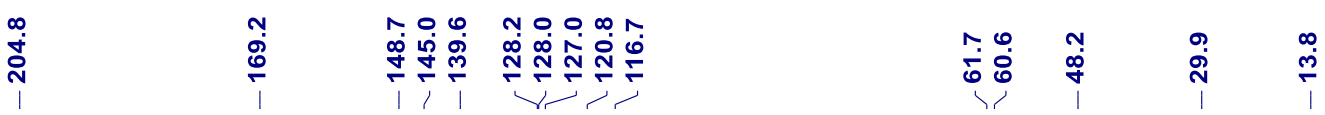

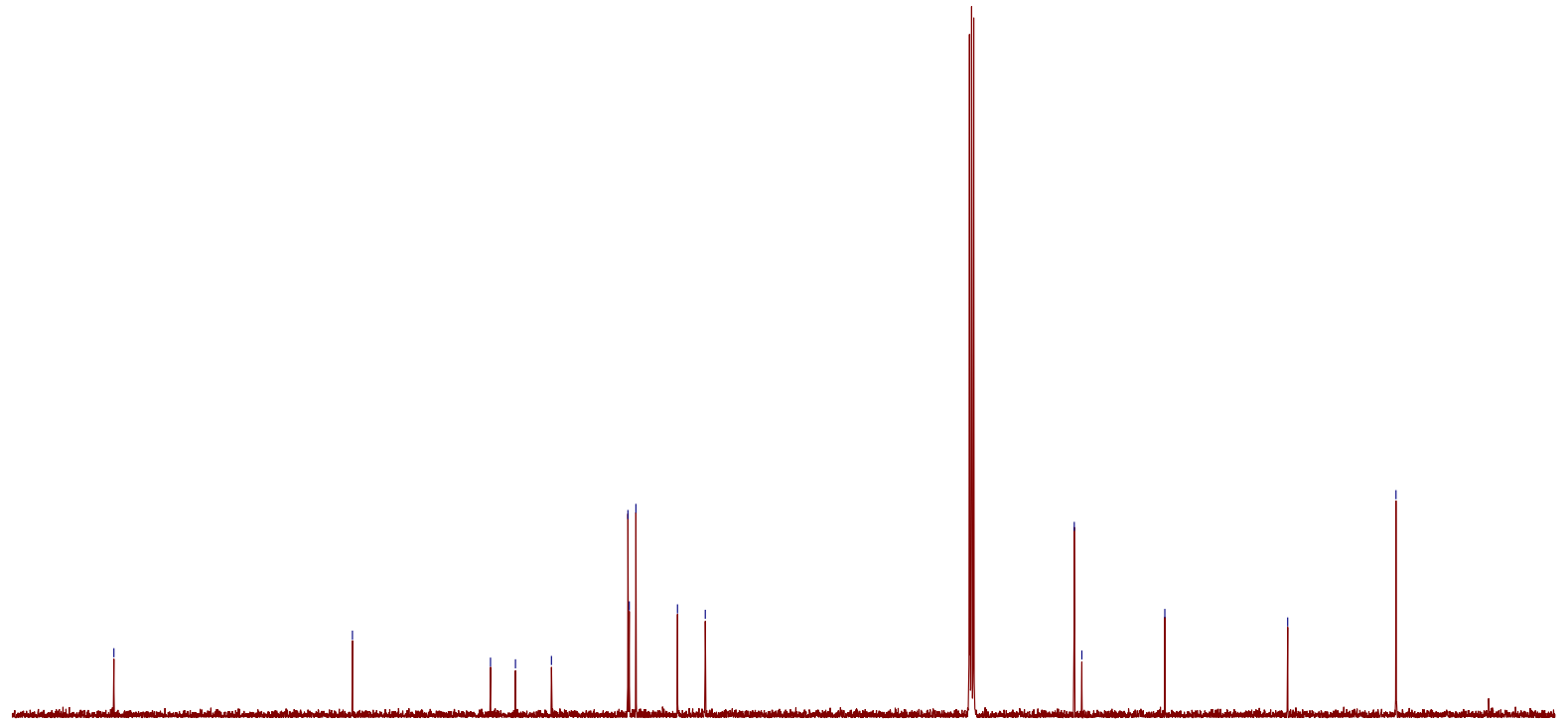

$\begin{array}{llllllllllllllllllllllll}20 & 210 & 200 & 190 & 180 & 170 & 160 & 150 & 140 & 130 & 120 & 110 & 100 & 90 & 80 & 70 & 60 & 50 & 40 & 30 & 20 & 10 & 0 & -\end{array}$ f1 (ppm)

${ }^{1} \mathrm{H}$ and ${ }^{13} \mathrm{C}$ NMR spectra for product $3 \mathrm{ae}\left(400 \mathrm{MHz}, \mathrm{CDCl}_{3}\right)$ 


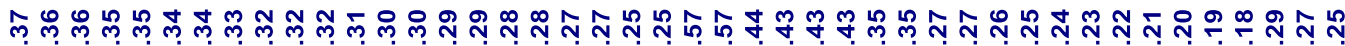

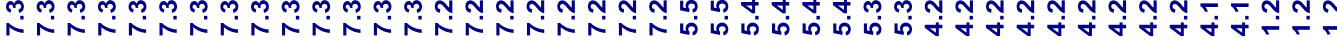<smiles>C=C(C(=C)C(F)(F)C(=O)OCC)c1ccccc1</smiles>

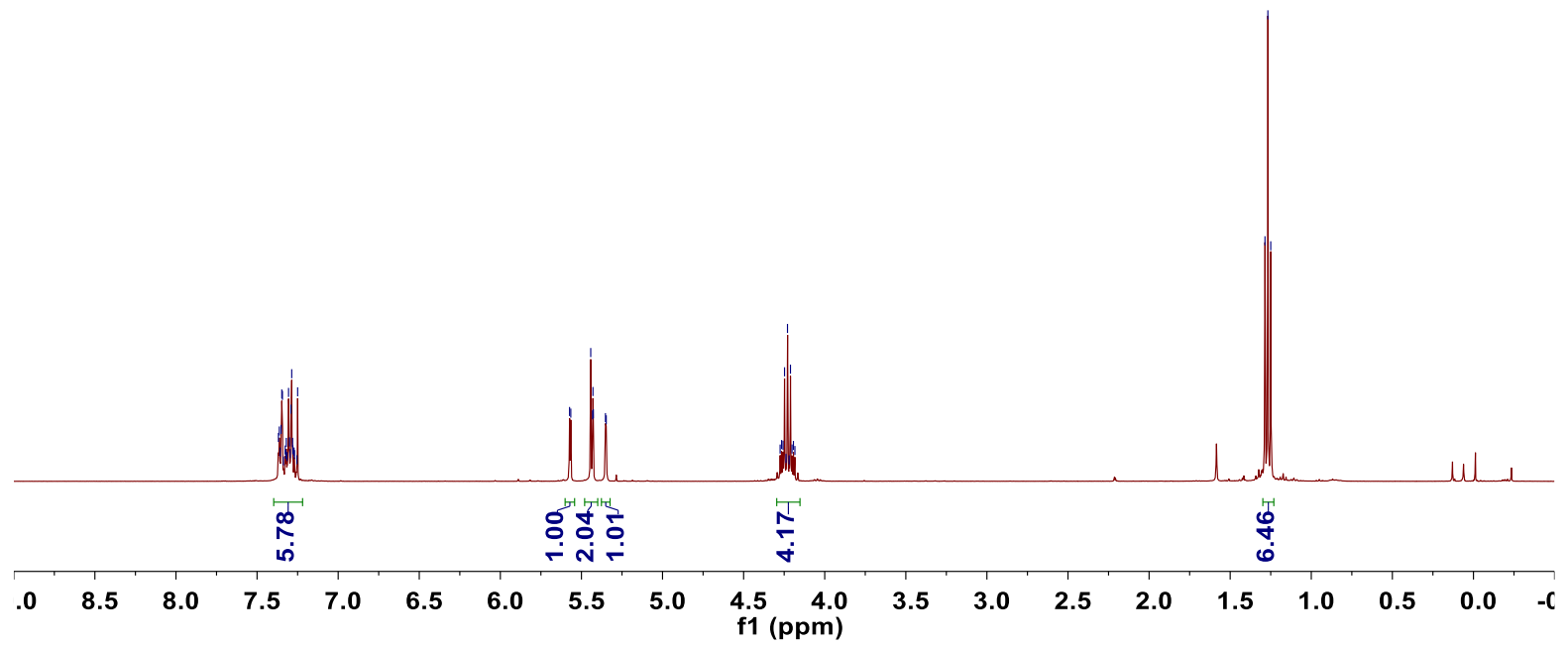

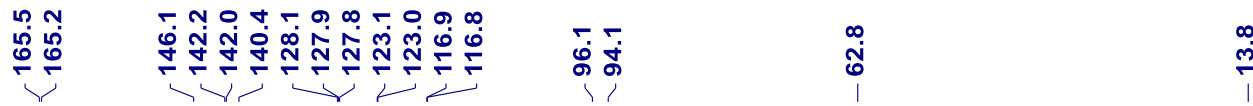

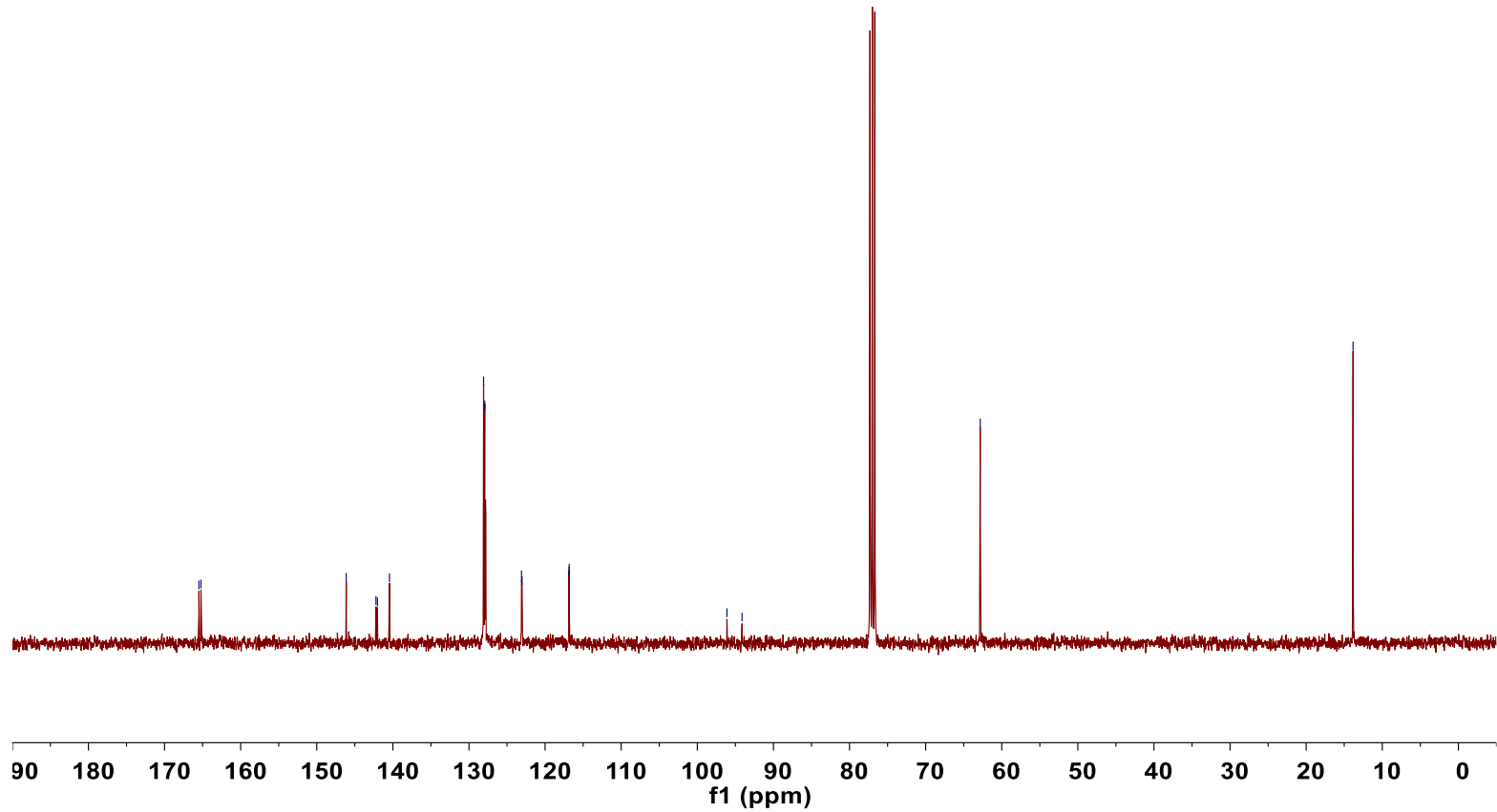




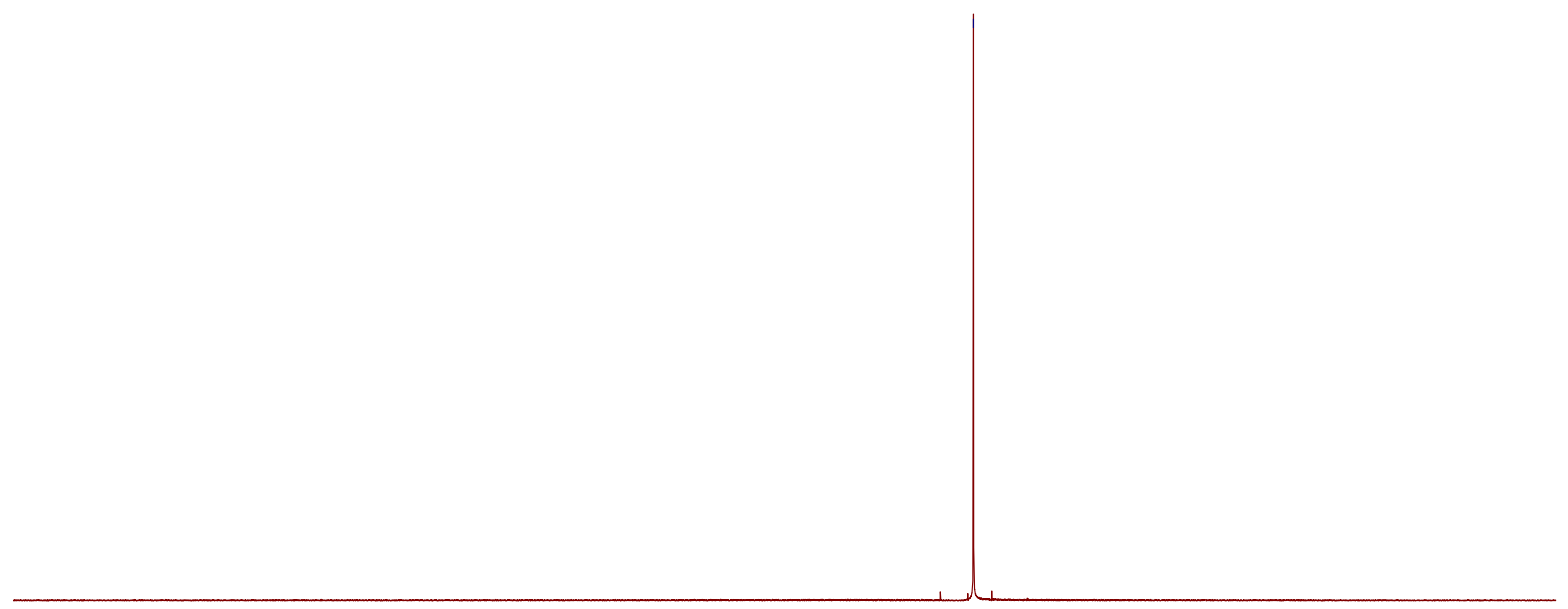
$\begin{array}{lllllllllllllllllllll}100 & 80 & 60 & 40 & 20 & 0 & -20 & -40 & -60 & -80 & -100 & -120 & -140 & -160 & -180 & -200 & -220 & -240 & -260 & -280 & -300\end{array}$

${ }^{1} \mathrm{H},{ }^{13} \mathrm{C}$ and ${ }^{19} \mathrm{~F}$ NMR spectra for product 3af $\left(400 \mathrm{MHz}, \mathrm{CDCl}_{3}\right)$ 


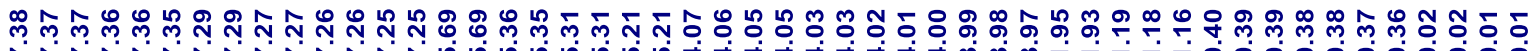

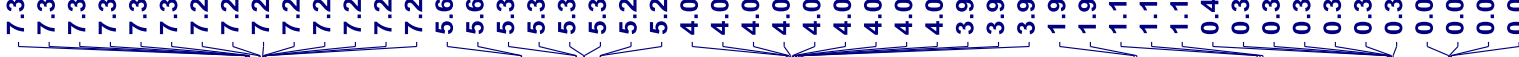<smiles>C=C(C(=C)C(CC1CC1)(OCC)C(=O)OCC)c1ccccc1</smiles>

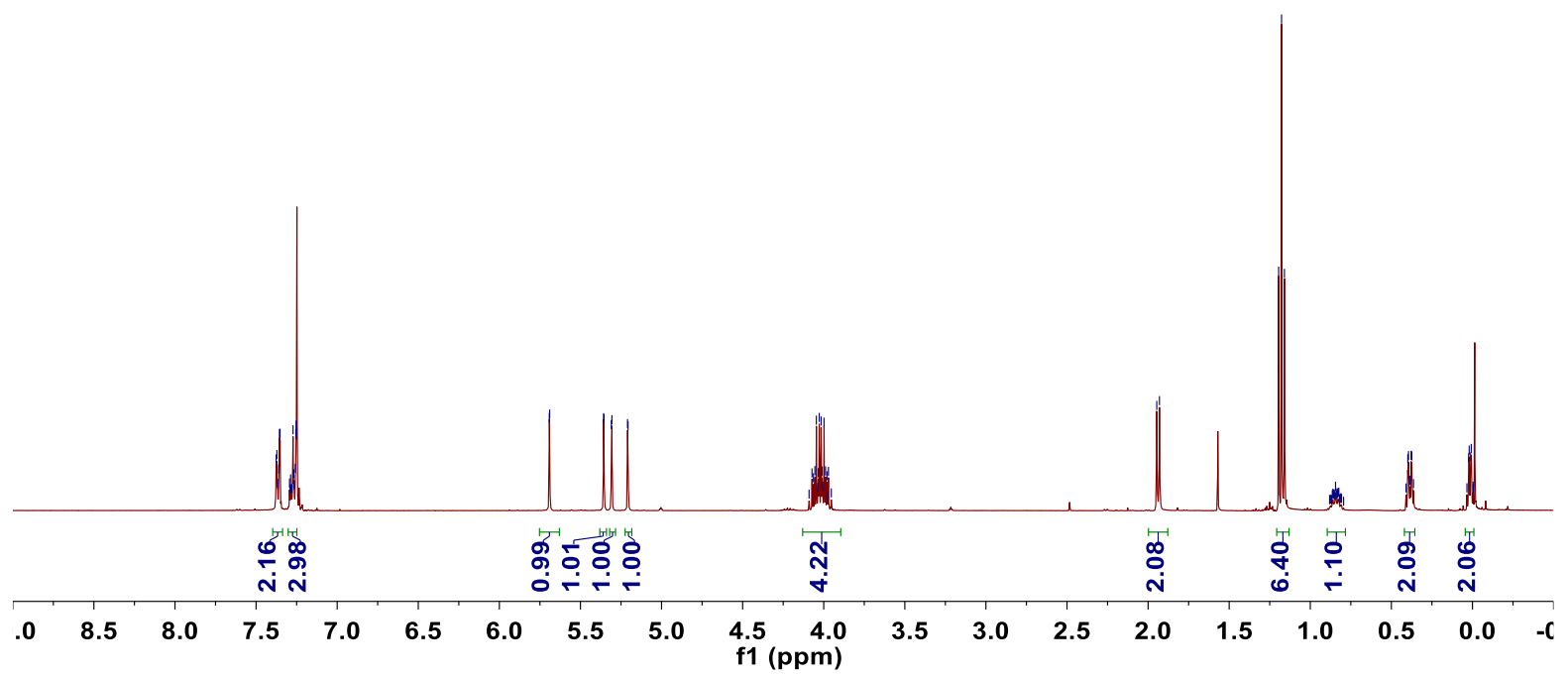

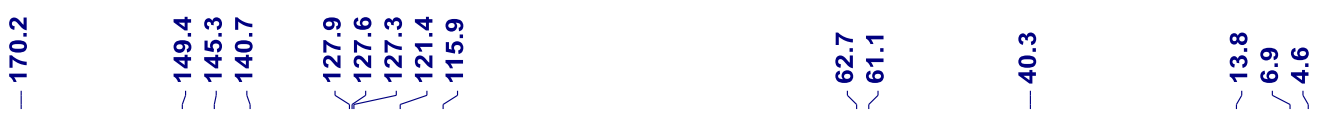

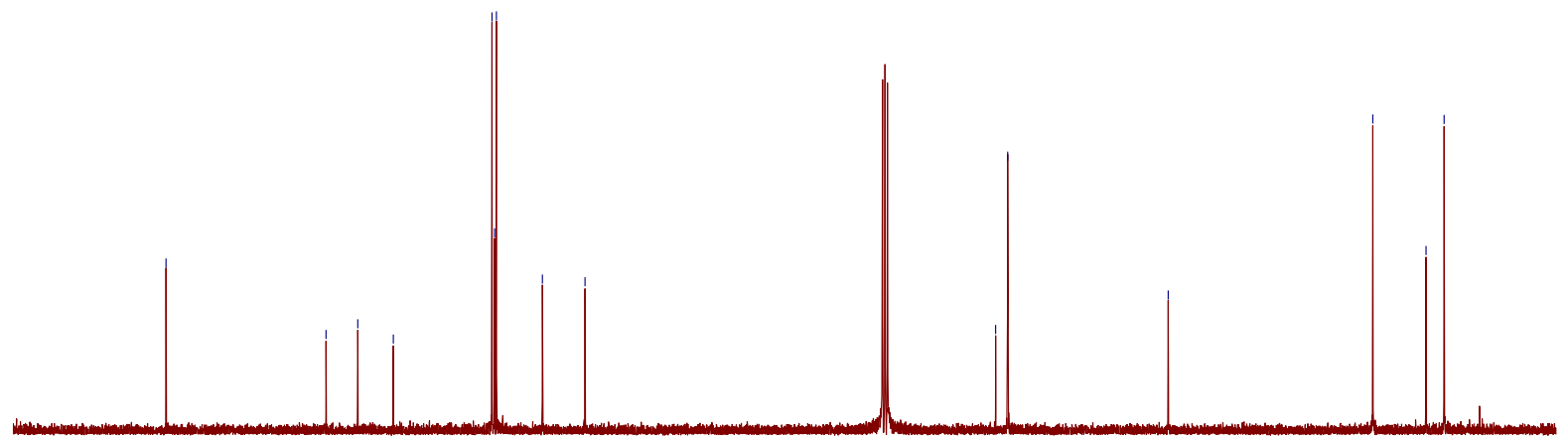

$\begin{array}{llllllllllllllllllll}180 & 170 & 160 & 150 & 140 & 130 & 120 & 110 & 100 & \underset{f 1}{(\mathrm{ppm})} & 80 & 70 & 60 & 50 & 40 & 30 & 20 & 10 & 0 & -\end{array}$

${ }^{1} \mathrm{H}$ and ${ }^{13} \mathrm{C}$ NMR spectra for product $3 \mathrm{ag}\left(400 \mathrm{MHz}, \mathrm{CDCl}_{3}\right)$ 
ผ

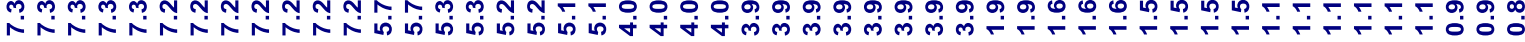<smiles>C=C(C(=C)C(C)(CC1CCCC1)OCC)c1ccccc1</smiles>

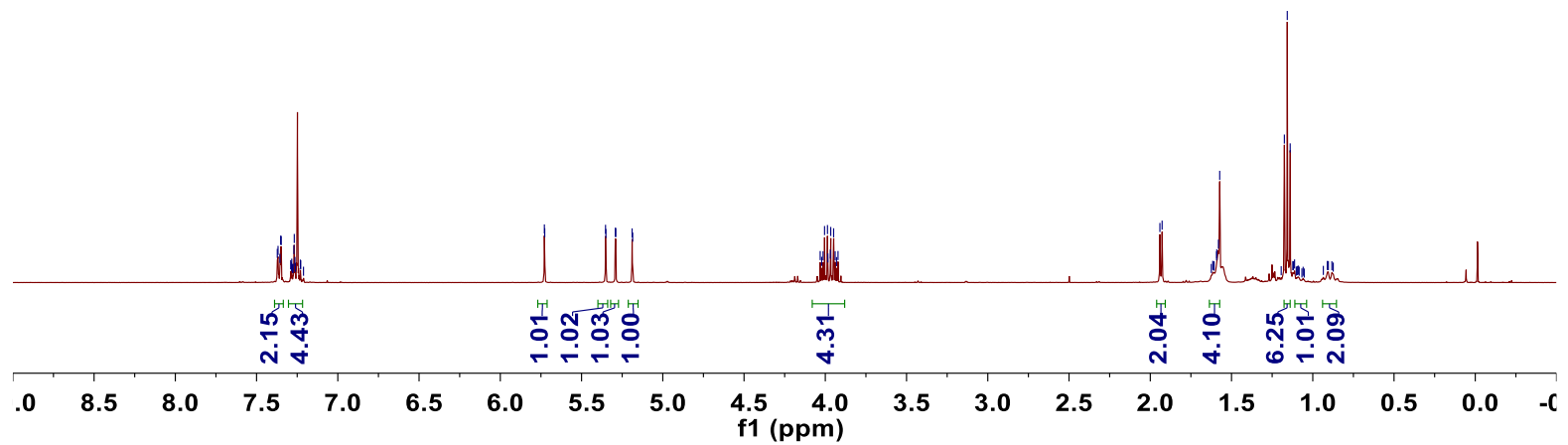

\begin{tabular}{|c|c|c|}
\hline 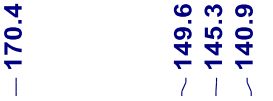 & 感怘占 & $\frac{\infty}{\square}$ \\
\hline
\end{tabular}

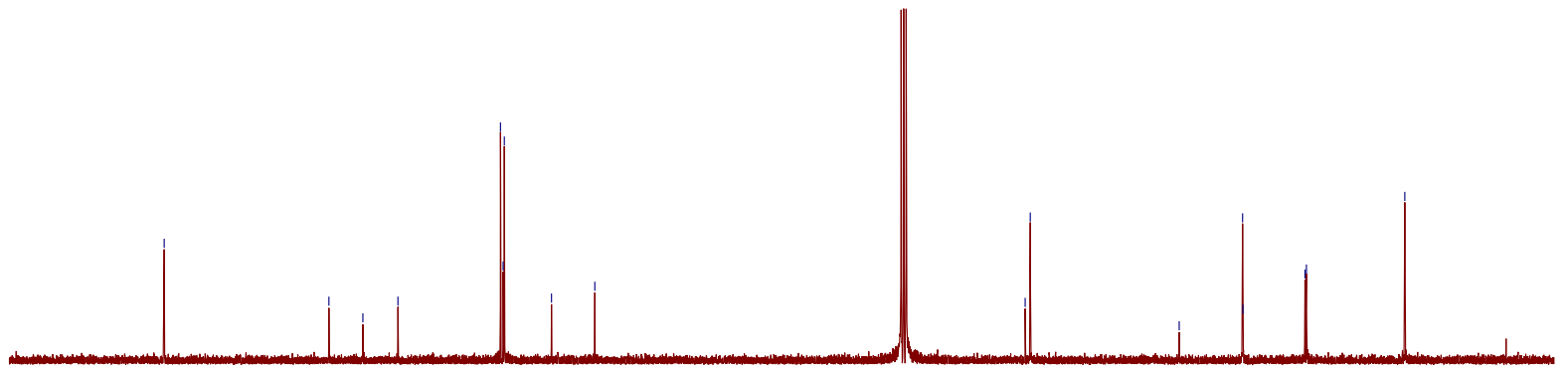

$\begin{array}{llllllllll}180 & 170 & 160 & 150 & 140 & 130 & 120 & 110 & \begin{array}{l}100 \\ \mathrm{f} 1(\mathrm{ppm})\end{array}\end{array}$

$40 \quad 30$

$2010 \quad 0$

${ }^{1} \mathrm{H}$ and ${ }^{13} \mathrm{C}$ NMR spectra for product $3 \mathrm{ah}\left(400 \mathrm{MHz}, \mathrm{CDCl}_{3}\right)$ 


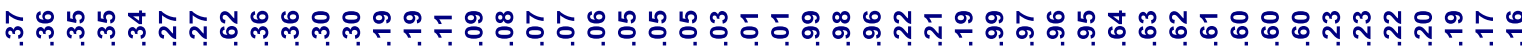

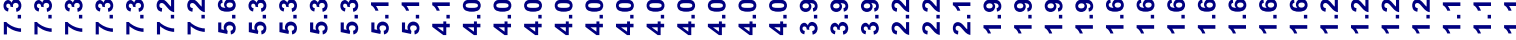<smiles>C=C(C(=C)C(C)(CCCOC(=O)CC)C(=O)OCC)c1ccccc1</smiles>

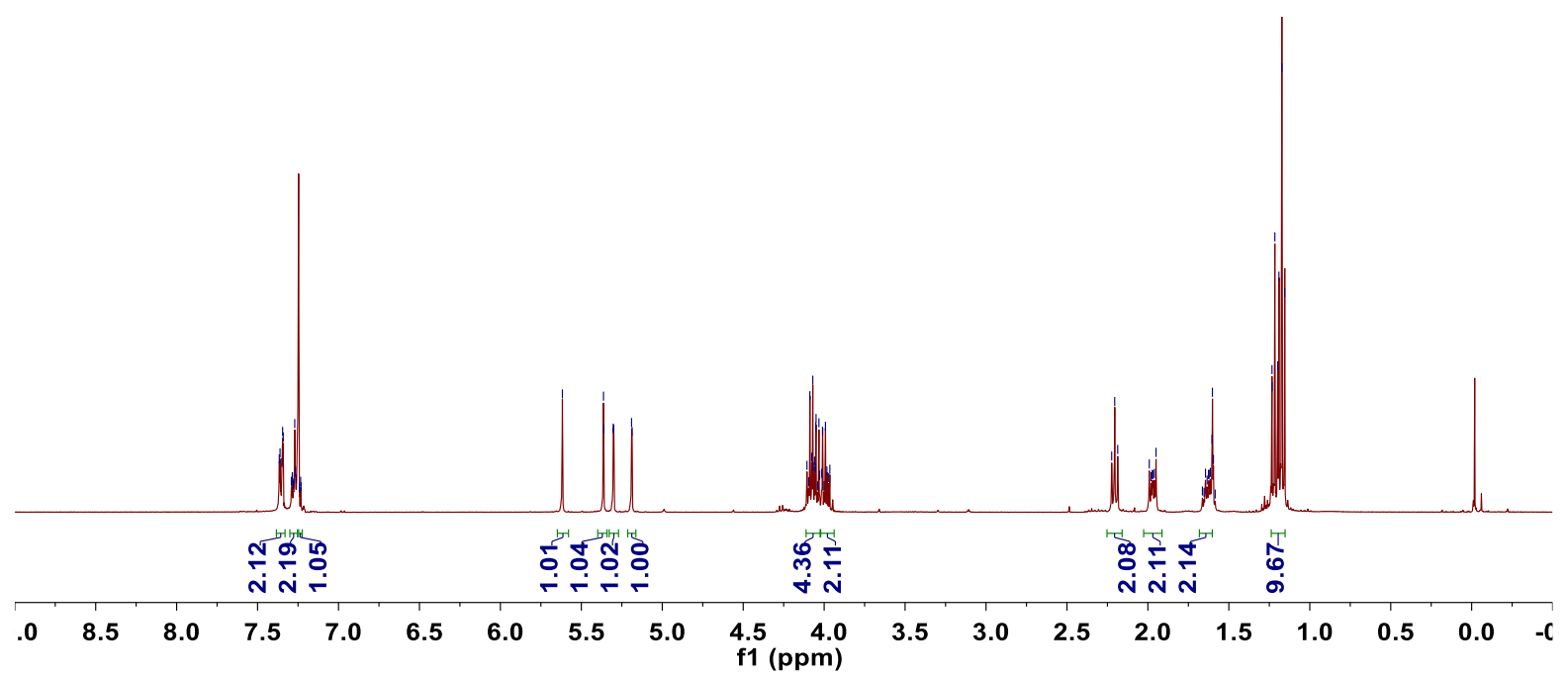

官是

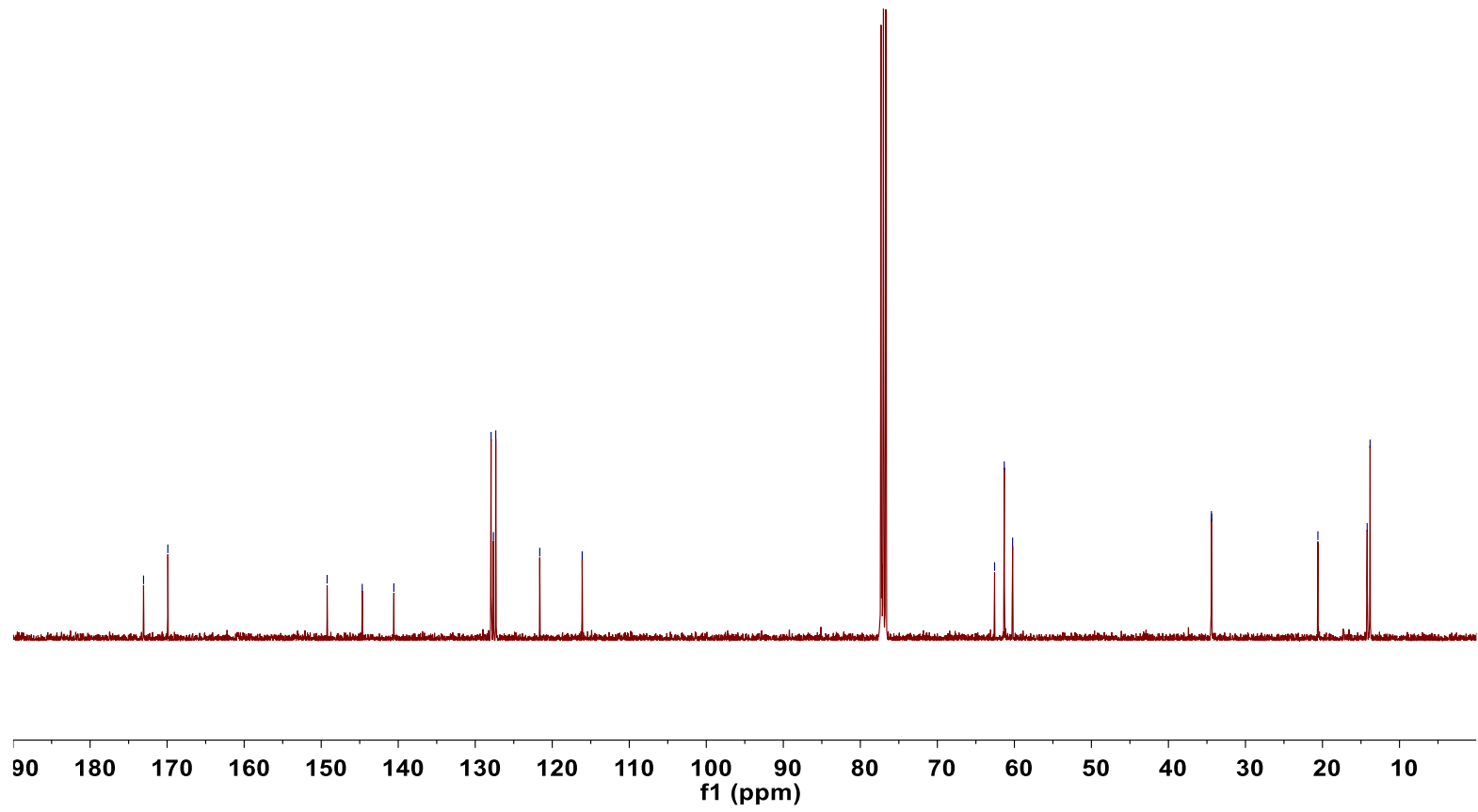

${ }^{1} \mathrm{H}$ and ${ }^{13} \mathrm{C}$ NMR spectra for product 3 ai $\left(400 \mathrm{MHz}, \mathrm{CDCl}_{3}\right)$ 


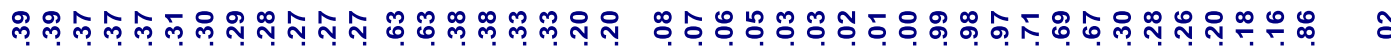

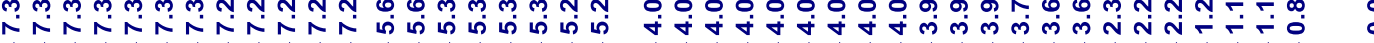<smiles>C=C(C(=C)C(CCOCC)(C(=O)OCC)C(=O)OCC)c1ccccc1</smiles>

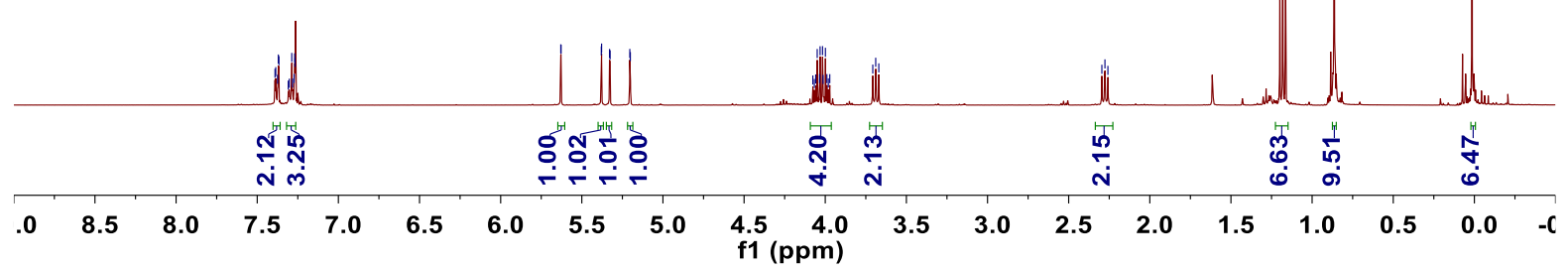

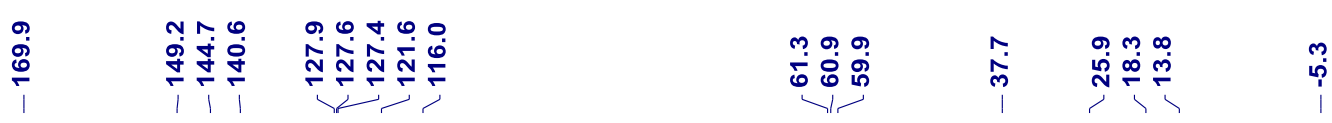

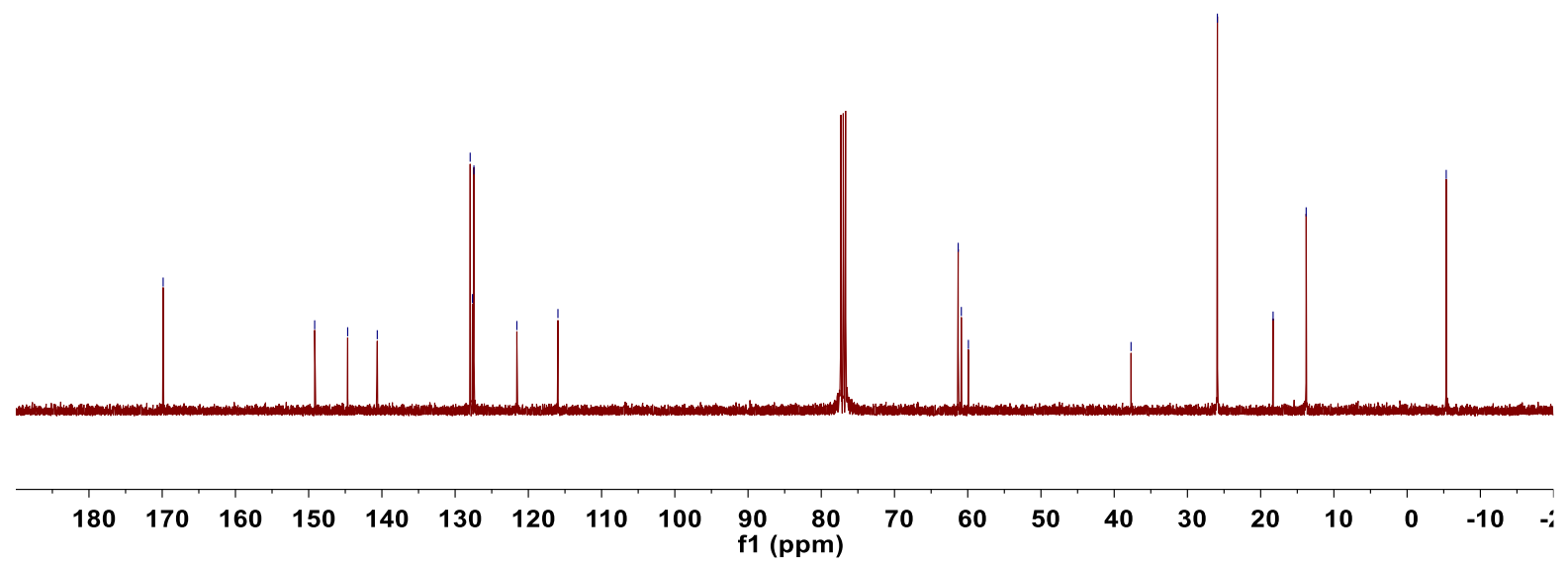

${ }^{1} \mathrm{H}$ and ${ }^{13} \mathrm{C}$ NMR spectra for product 3aj (400 $\left.\mathrm{MHz}, \mathrm{CDCl}_{3}\right)$ 


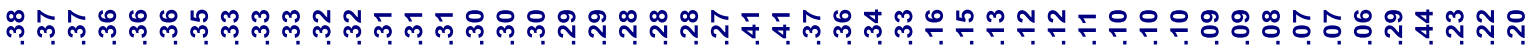

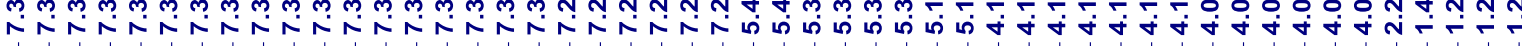<smiles>C=C(C(=C)C(C)(C)C(C)=O)c1ccccc1</smiles>

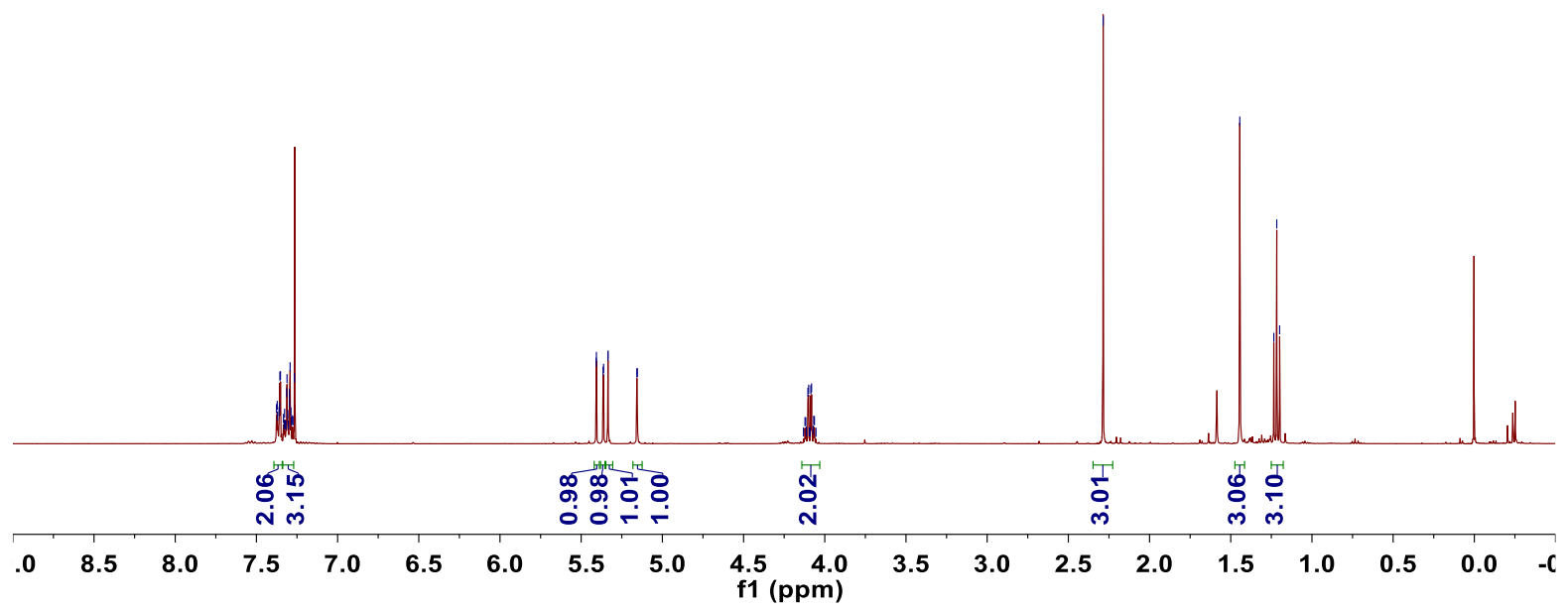

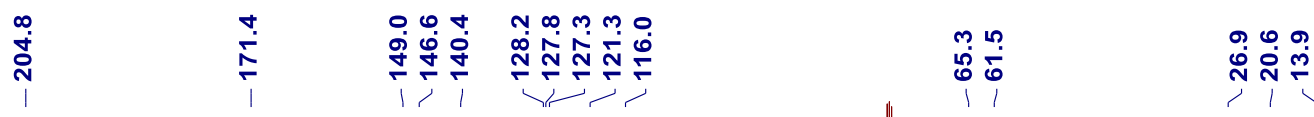

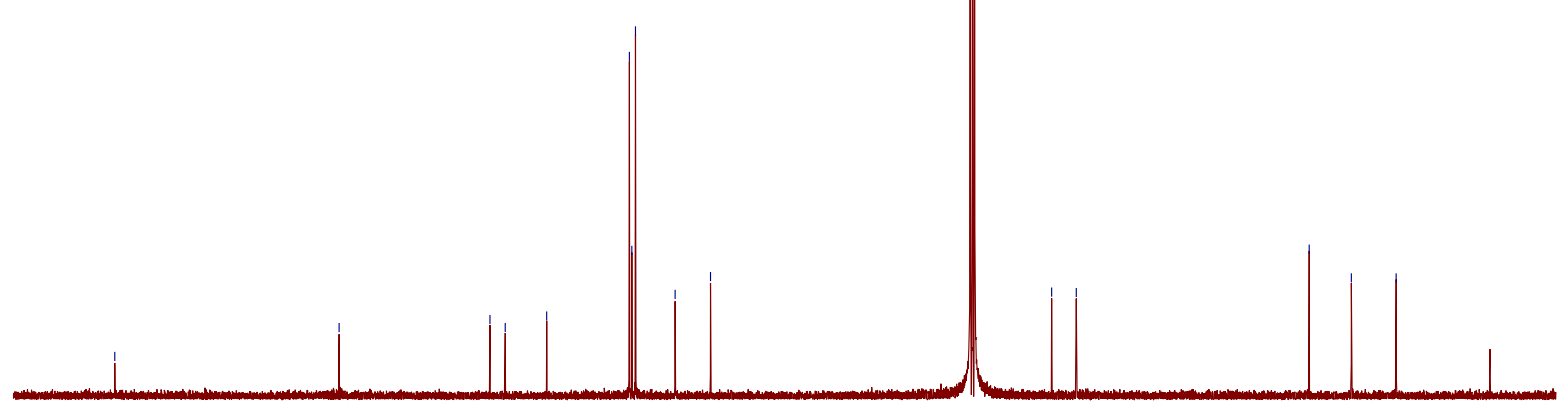

$\begin{array}{lllllllllllllllllllllllllll}20 & 210 & 200 & 190 & 180 & 170 & 160 & 150 & 140 & 130 & 120 & 110 & 100 & 90 & 80 & 70 & 60 & 50 & 40 & 30 & 20 & 10 & 0 & -\end{array}$ f1 (ppm)

${ }^{1} \mathrm{H}$ and ${ }^{13} \mathrm{C}$ NMR spectra for product $3 \mathrm{ak}\left(400 \mathrm{MHz}, \mathrm{CDCl}_{3}\right)$ 


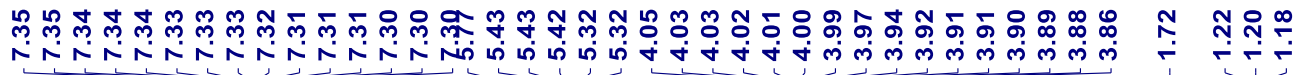

$\overbrace{\mathrm{CN}}^{\mathrm{Me}}$

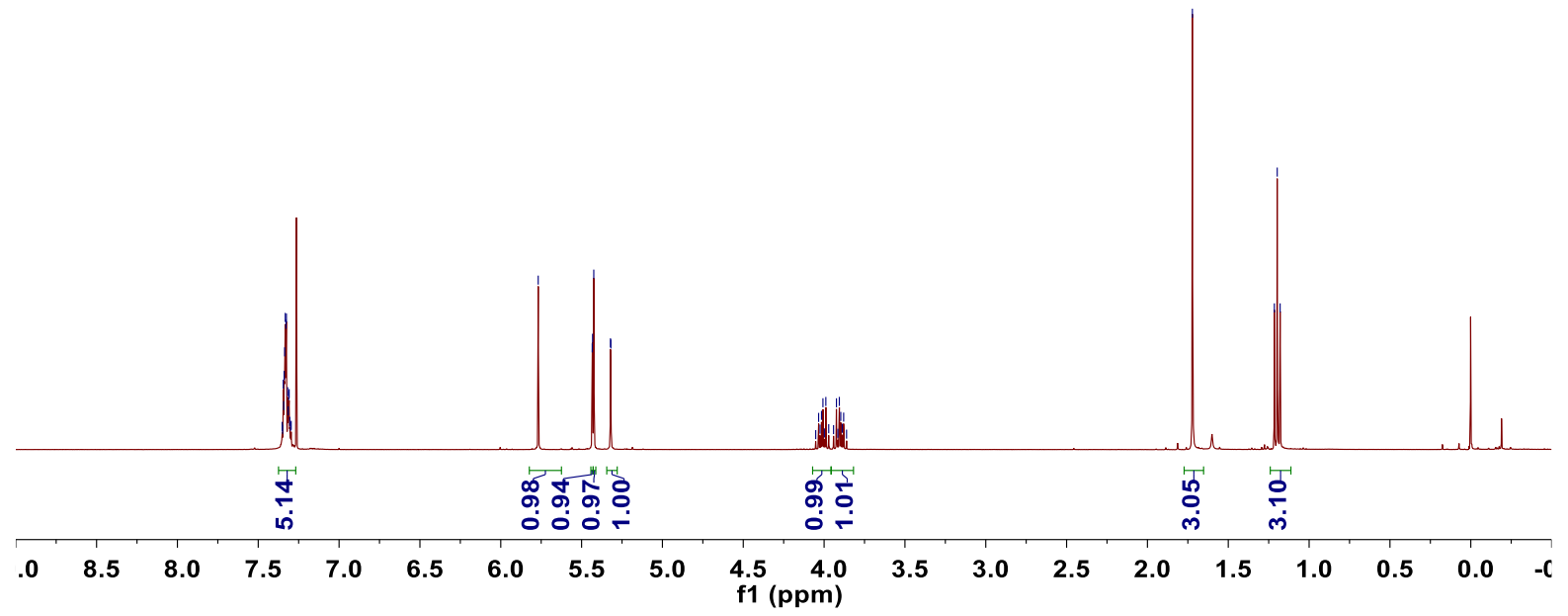

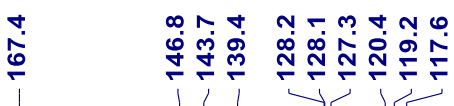

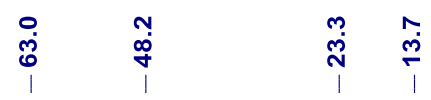

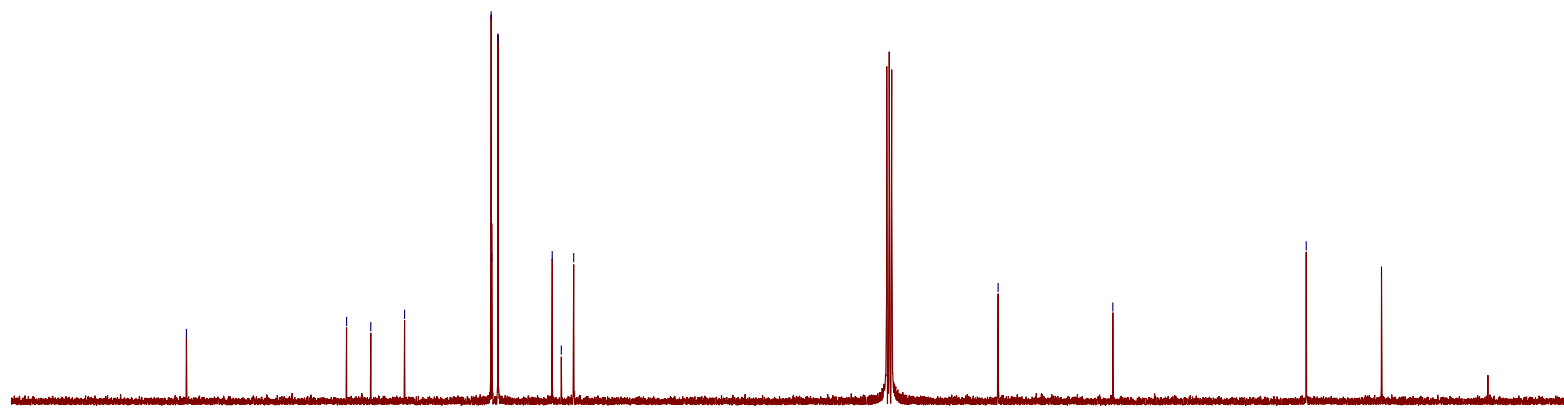

$\begin{array}{lllllllllll}90 & 180 & 170 & 160 & 150 & 140 & 130 & 120 & 110 & 100 \underset{\mathrm{f} 1}{90}(\mathrm{ppm}) & 80\end{array}$

${ }^{1} \mathrm{H}$ and ${ }^{13} \mathrm{C}$ NMR spectra for product $3 \mathrm{al}\left(400 \mathrm{MHz}, \mathrm{CDCl}_{3}\right)$ 


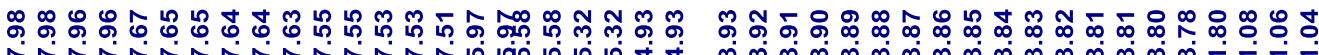

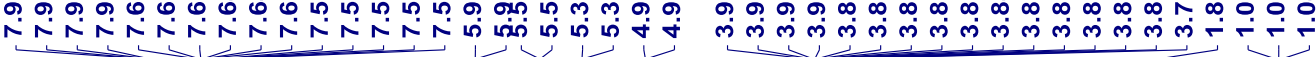<smiles>C=C(C(=C)C(C)([18O]OCC)c1ccccc1)C(=O)OCC</smiles>

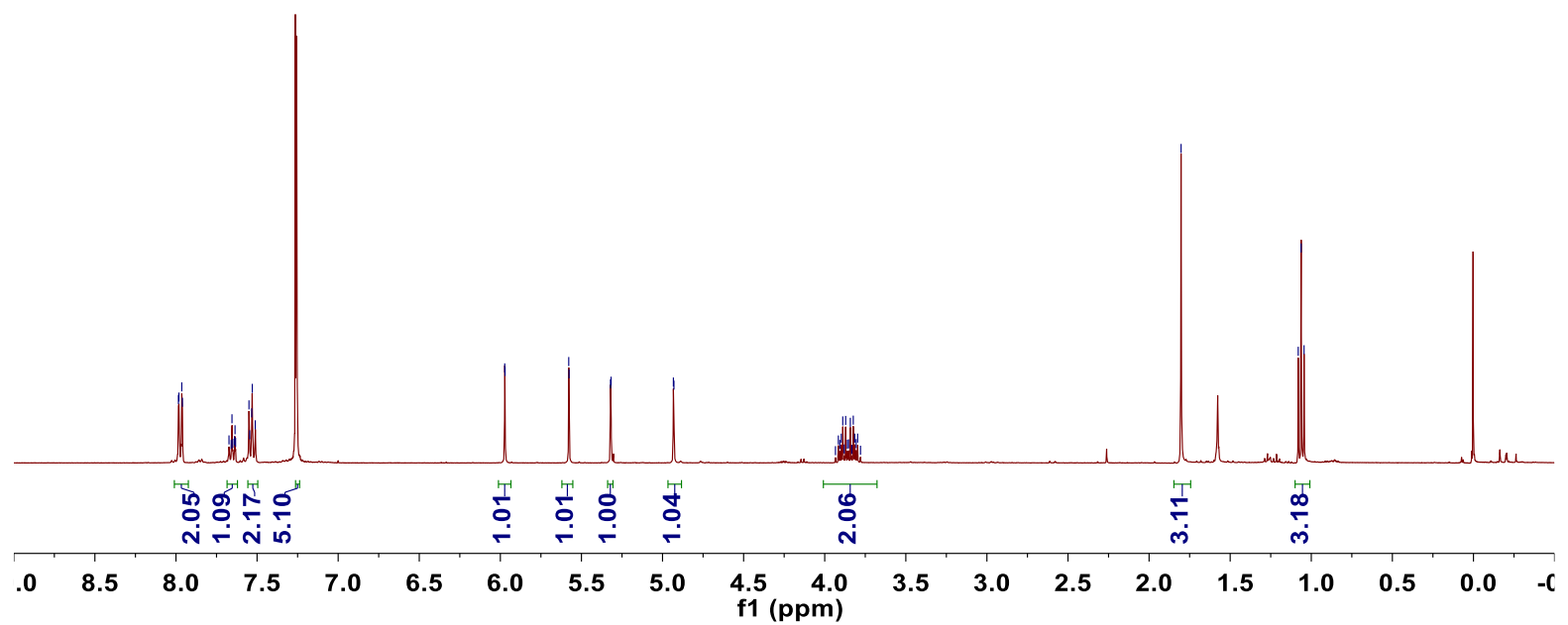

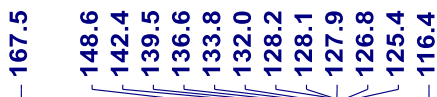

$\stackrel{\substack{n \\ 0}}{1} \quad$ กู

กั

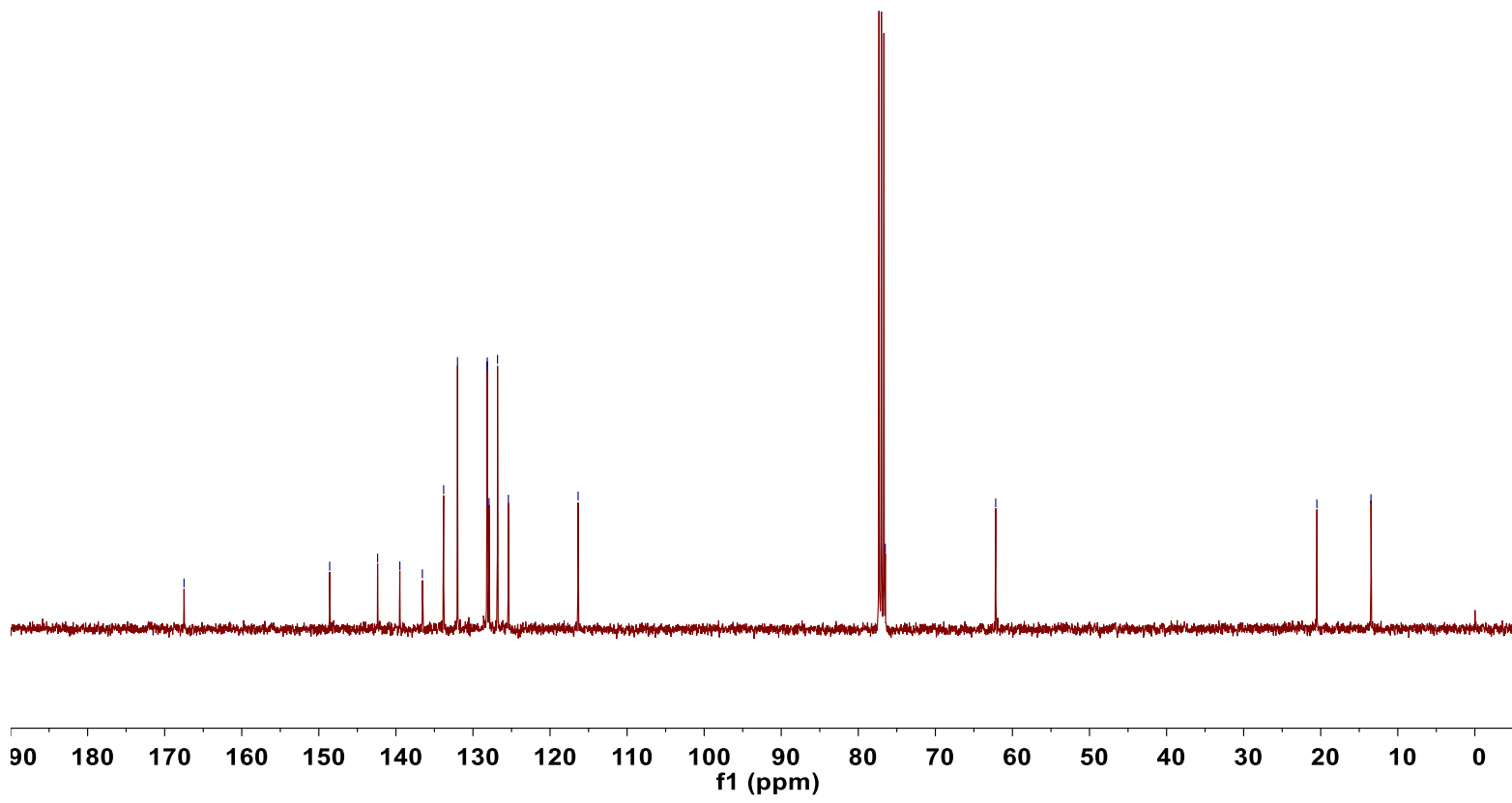

${ }^{1} \mathrm{H}$ and ${ }^{13} \mathrm{C}$ NMR spectra for product $3 \mathrm{am}\left(400 \mathrm{MHz}, \mathrm{CDCl}_{3}\right)$ 


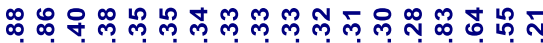

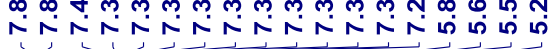

$\stackrel{\infty}{\stackrel{\infty}{i}} \stackrel{\stackrel{0}{\leftarrow}}{i}$

$\overbrace{\mathrm{Ts}}^{\mathrm{Me}}$

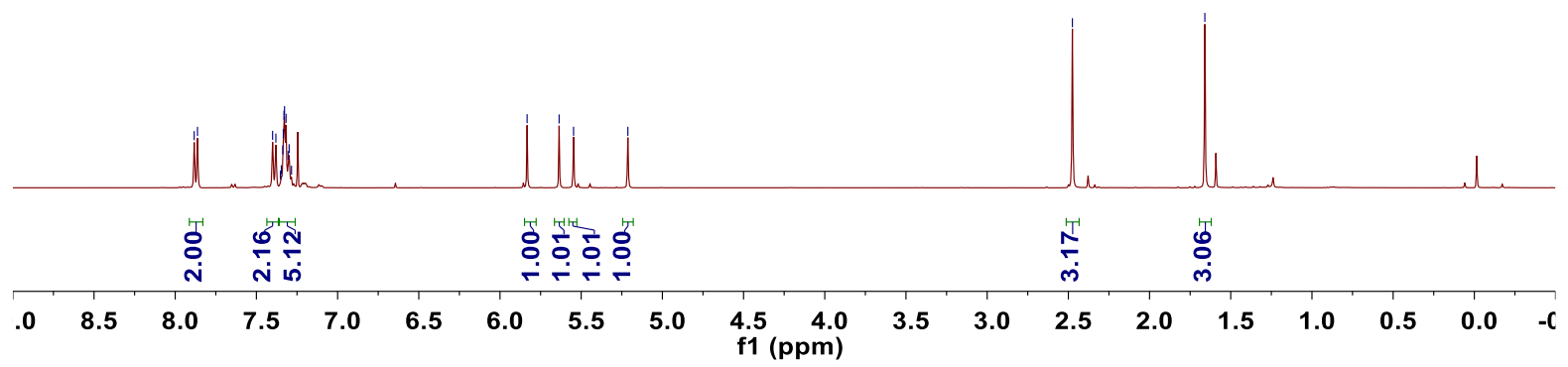

น พุ

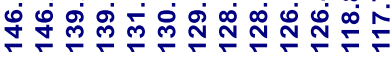

กิ่

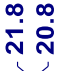

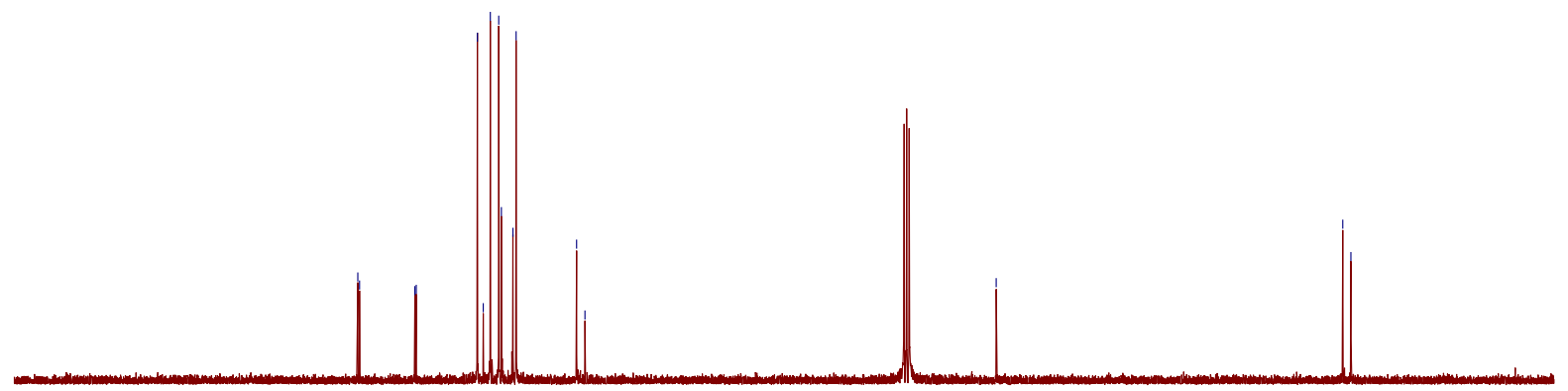

$\begin{array}{lllllllllll}90 & 180 & 170 & 160 & 150 & 140 & 130 & 120 & 110 & \begin{array}{c}100 \\ \mathrm{f} 1(\mathrm{ppm})\end{array}\end{array}$

$\begin{array}{lllllllll}80 & 70 & 60 & 50 & 40 & 30 & 20 & 10 & 0\end{array}$

${ }^{1} \mathrm{H}$ and ${ }^{13} \mathrm{C}$ NMR spectra for product $3 a n\left(400 \mathrm{MHz}, \mathrm{CDCl}_{3}\right)$ 


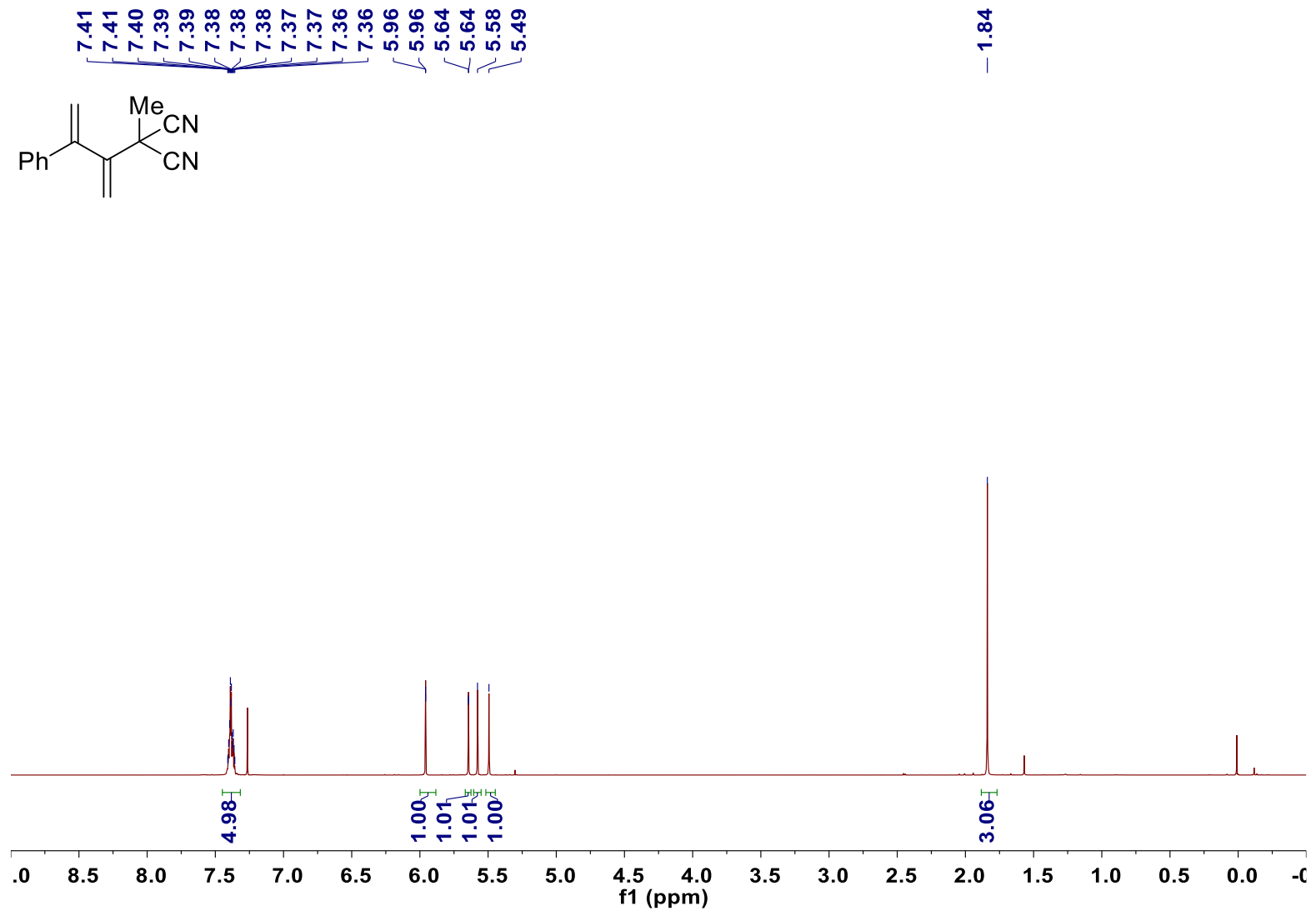

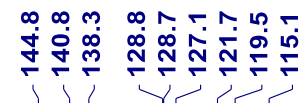

$\stackrel{m}{\dot{m}} \quad \stackrel{0}{\dot{0}}$

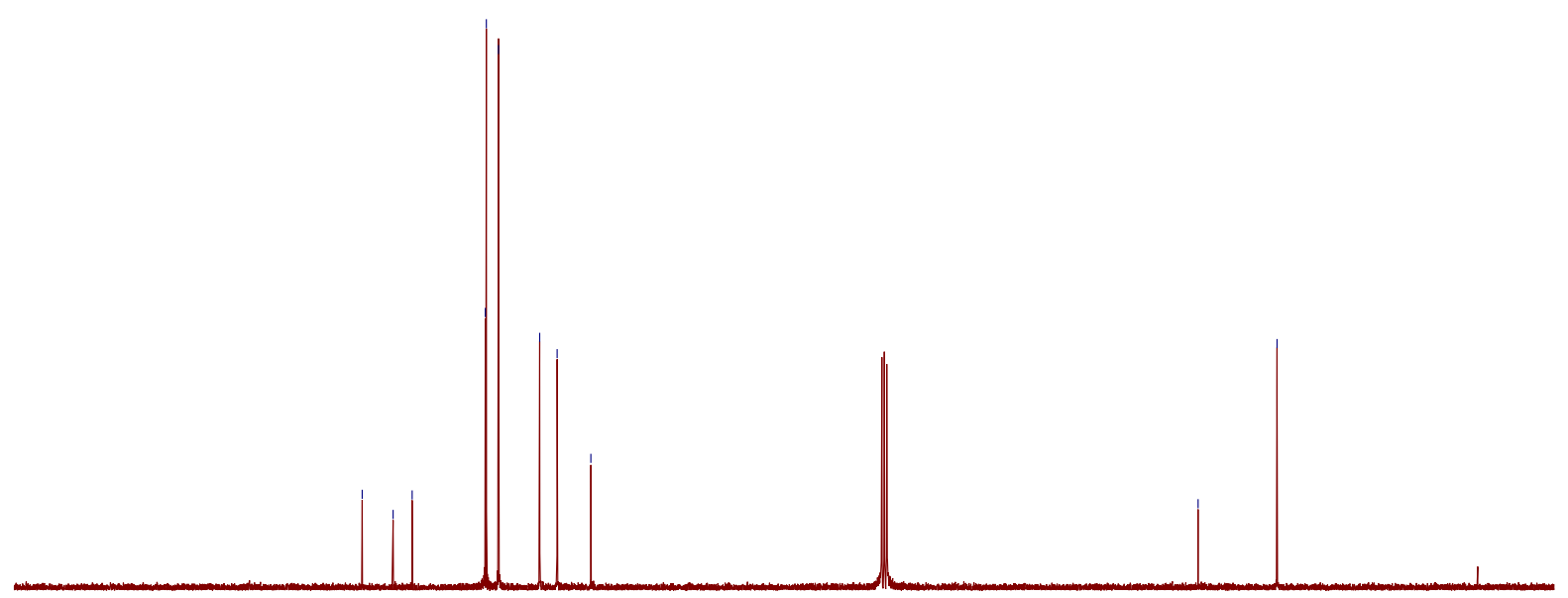

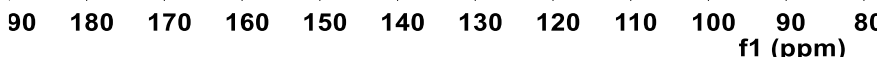

${ }^{1} \mathrm{H}$ and ${ }^{13} \mathrm{C}$ NMR spectra for product $3 \mathrm{ao}\left(400 \mathrm{MHz}, \mathrm{CDCl}_{3}\right)$ 

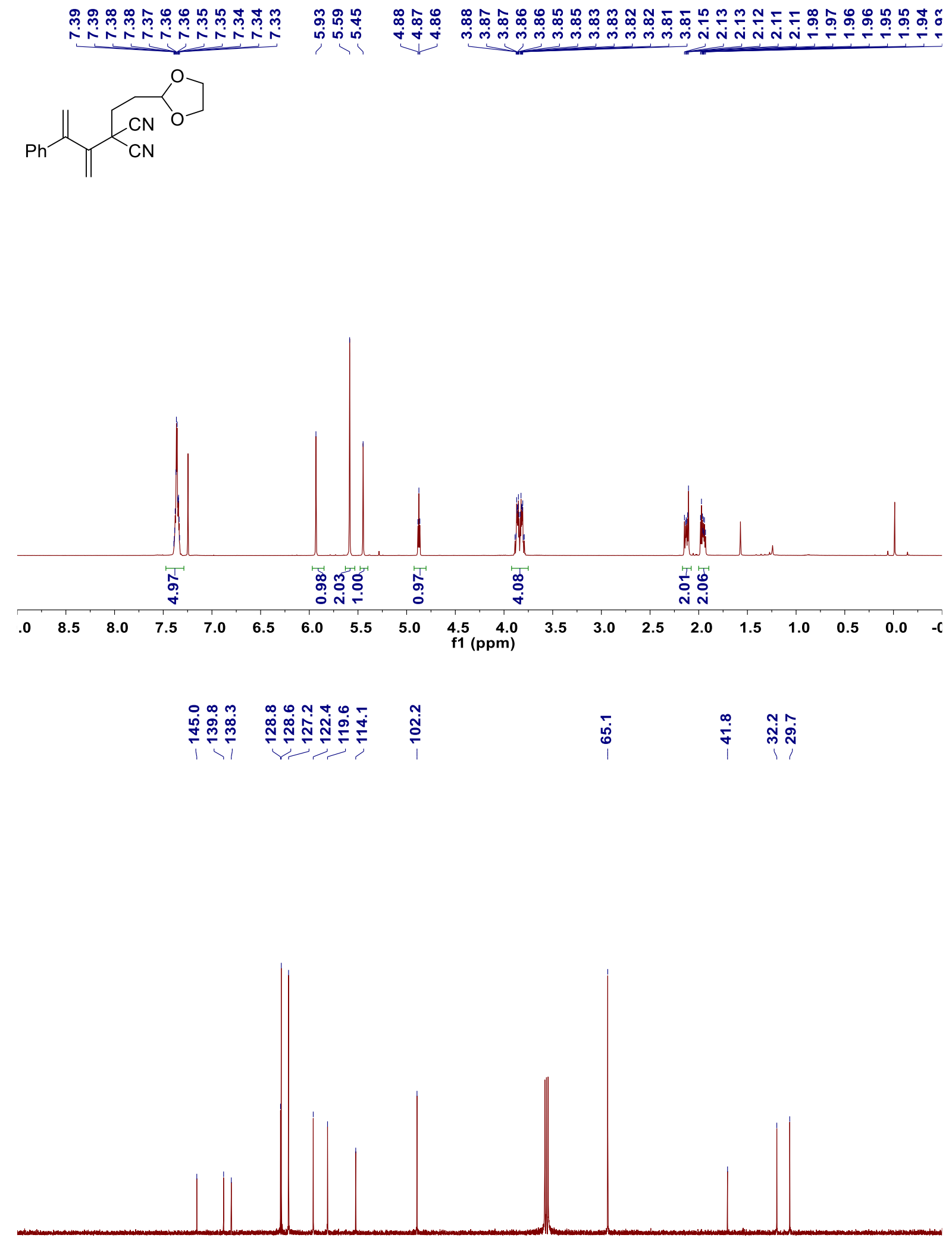

80

$\begin{array}{lllllllll}170 & 160 & 150 & 140 & 130 & 120 & 110 & 100 \underset{f 1}{90}(\mathrm{ppm}) & 80\end{array}$

${ }^{1} \mathrm{H}$ and ${ }^{13} \mathrm{C}$ NMR spectra for product $3 a p\left(400 \mathrm{MHz}, \mathrm{CDCl}_{3}\right)$ 


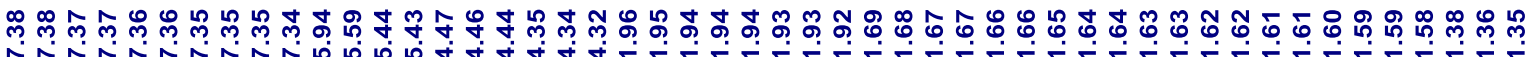

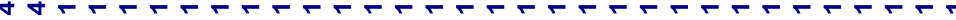<smiles>C=C(C(=C)C(C)(C#N)CCCCCF)c1ccccc1</smiles>

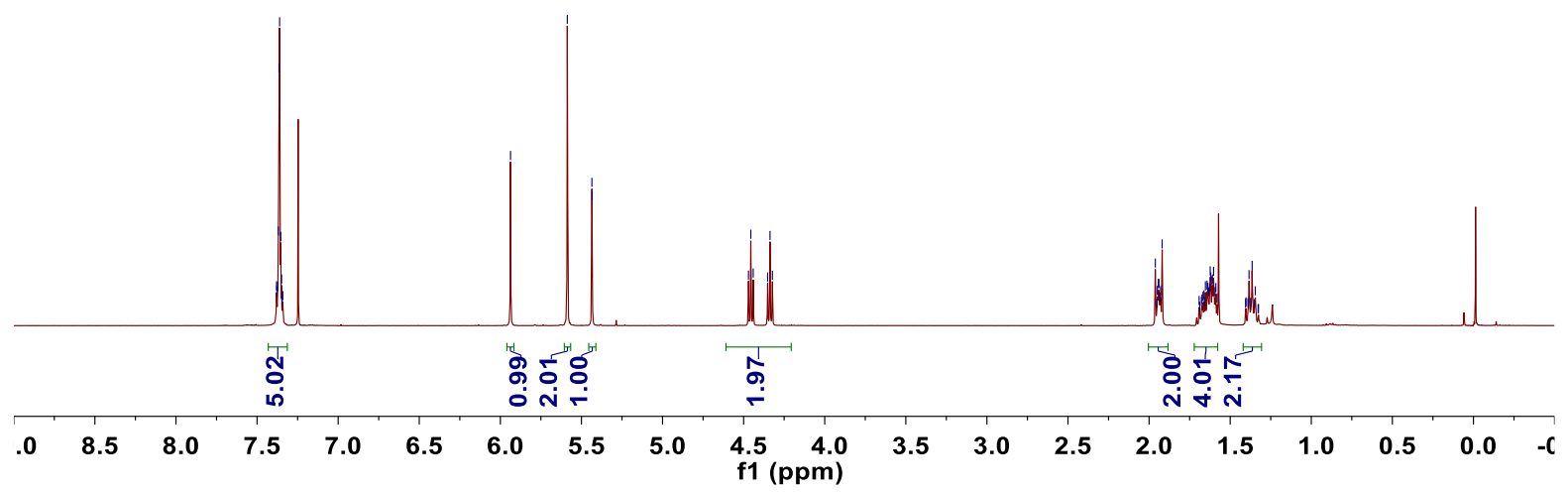

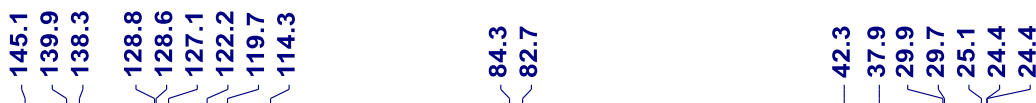

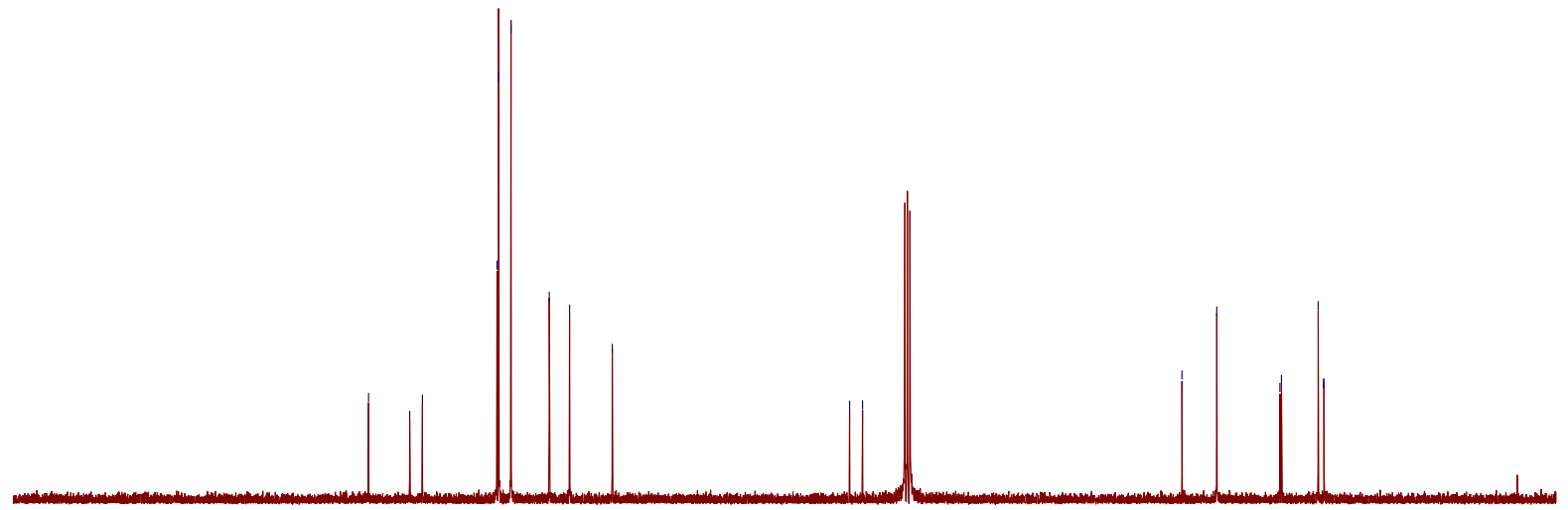

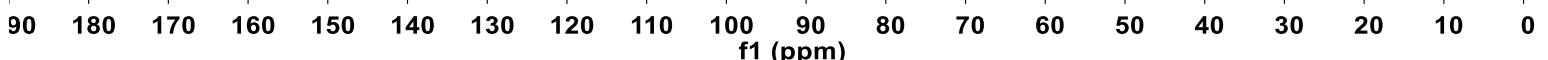




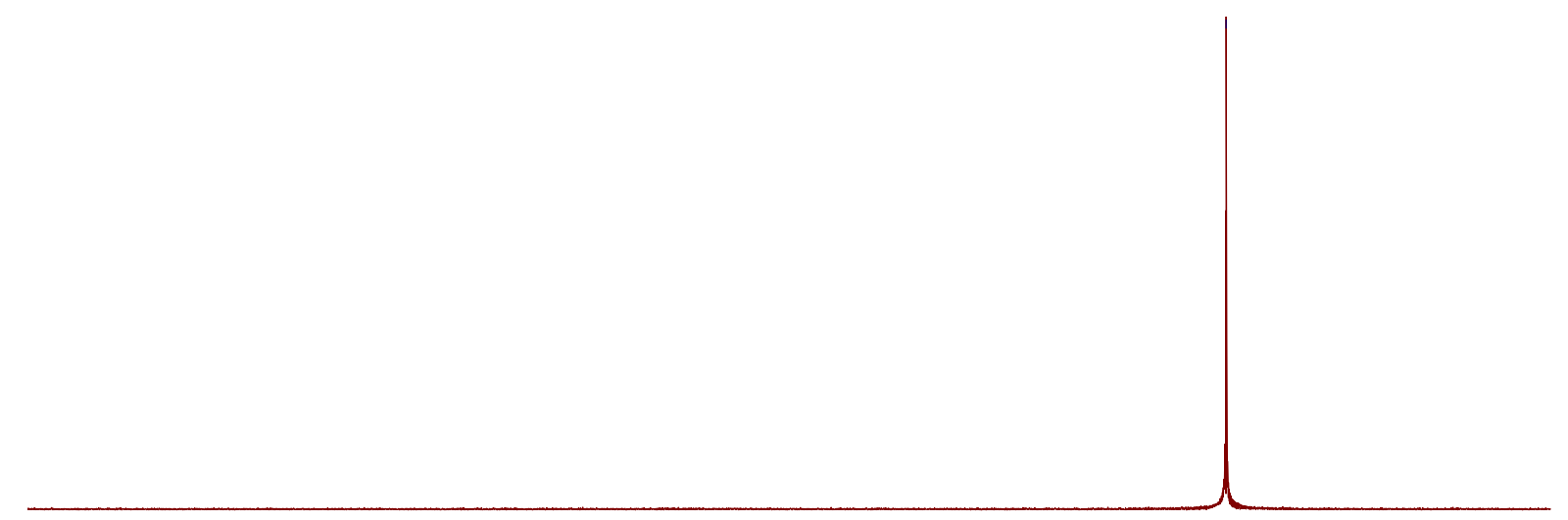

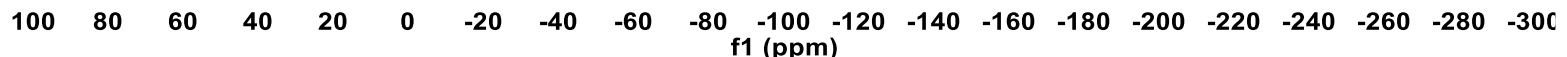

${ }^{1} \mathrm{H},{ }^{13} \mathrm{C}$ and ${ }^{19} \mathrm{~F}$ NMR spectra for product 3aq (400 $\left.\mathrm{MHz}, \mathrm{CDCl}_{3}\right)$ 


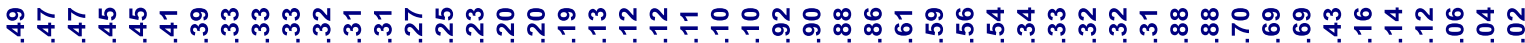

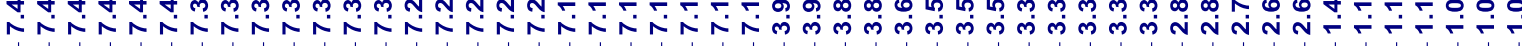<smiles>CCOC(=O)C(C)(CC)C1=C(c2ccccc2)CC2C(=O)N(c3ccccc3)C(=O)C2C1</smiles>

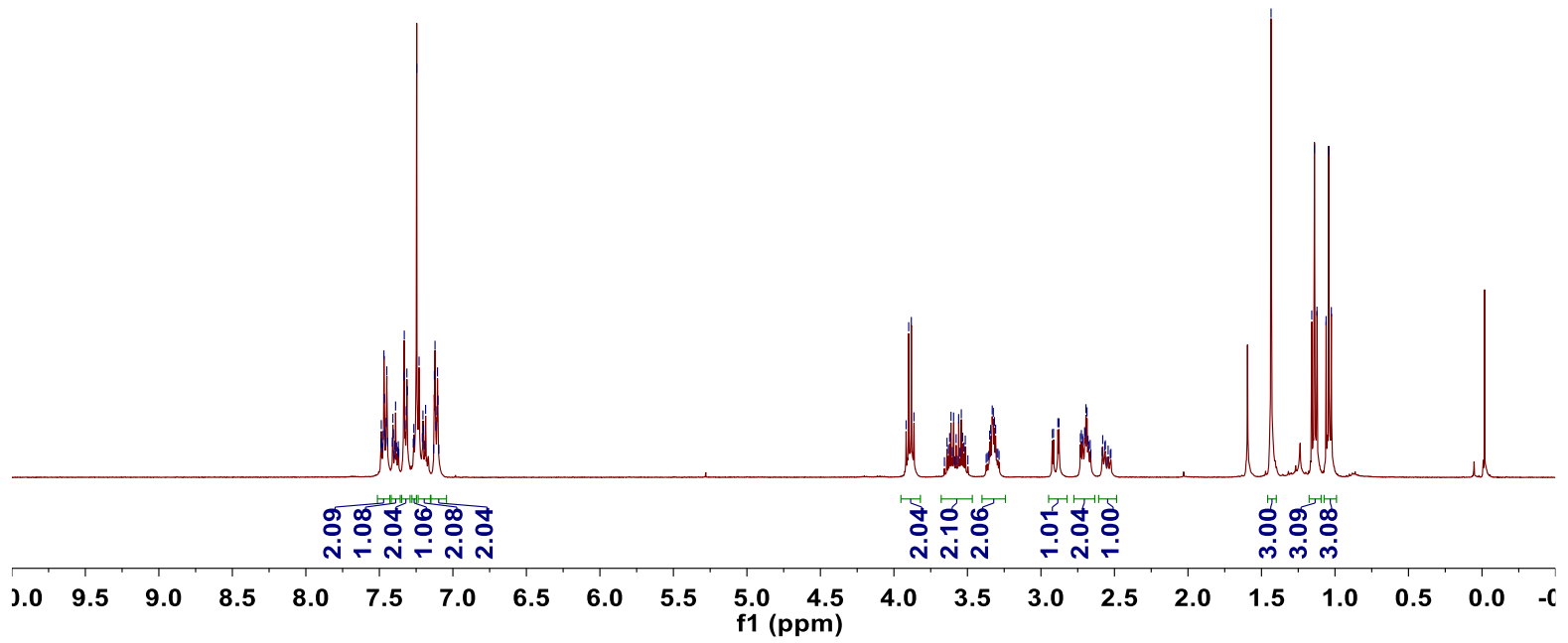

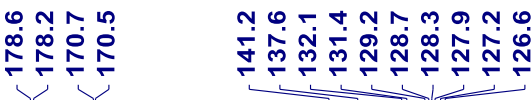

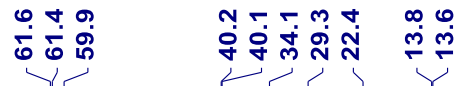

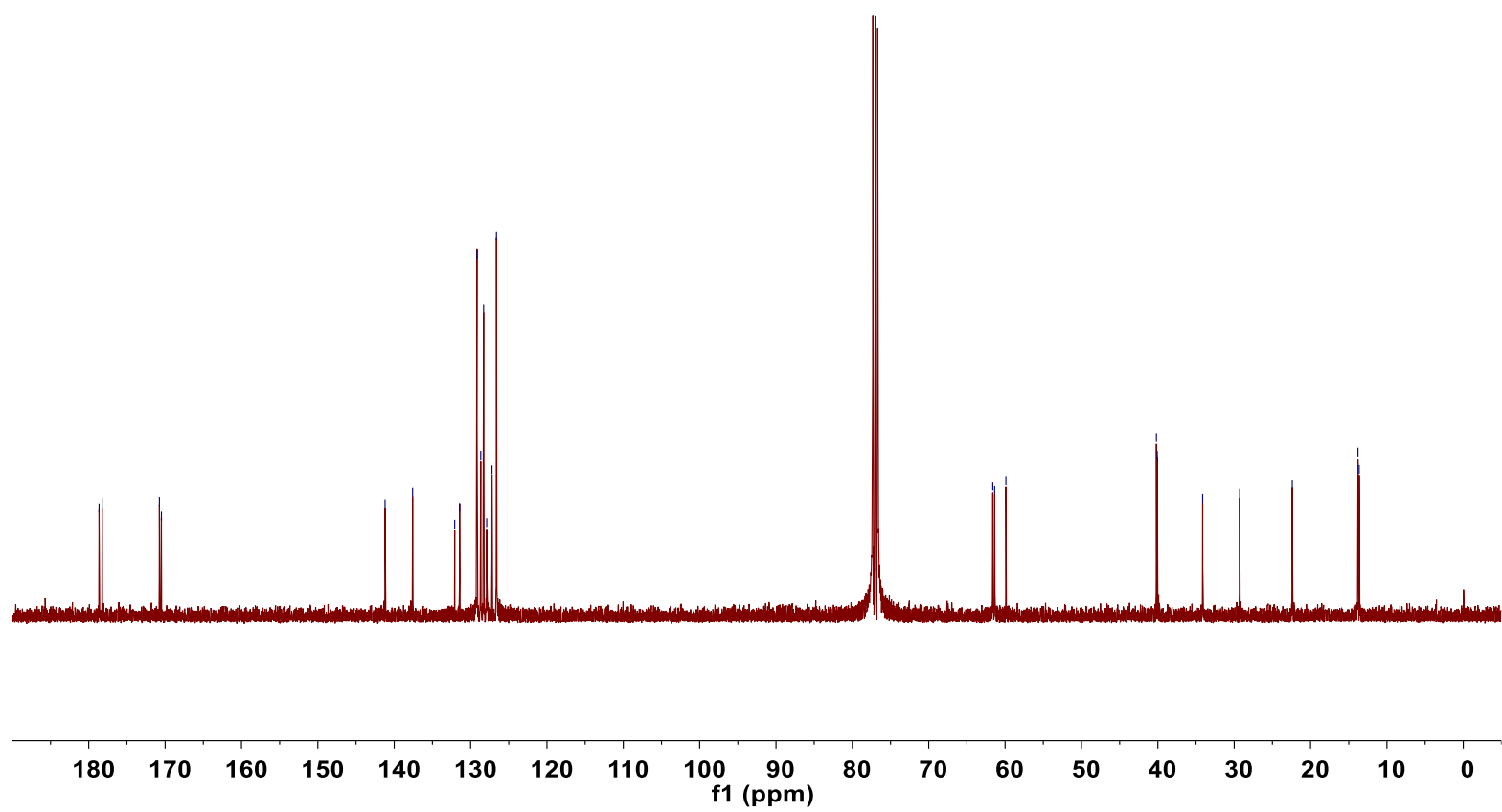

${ }^{1} \mathrm{H}$ and ${ }^{13} \mathrm{C}$ NMR spectra for product $4\left(400 \mathrm{MHz}, \mathrm{CDCl}_{3}\right)$ 


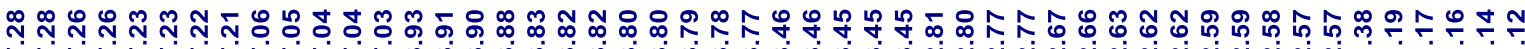

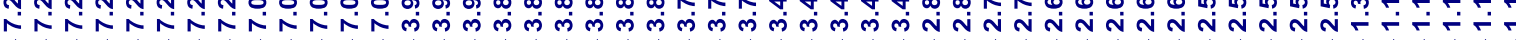<smiles>CCOC(=O)C(C)(C(=O)OCC)C1=C(c2ccccc2)CC2C(=O)OC(=O)C2C1</smiles>

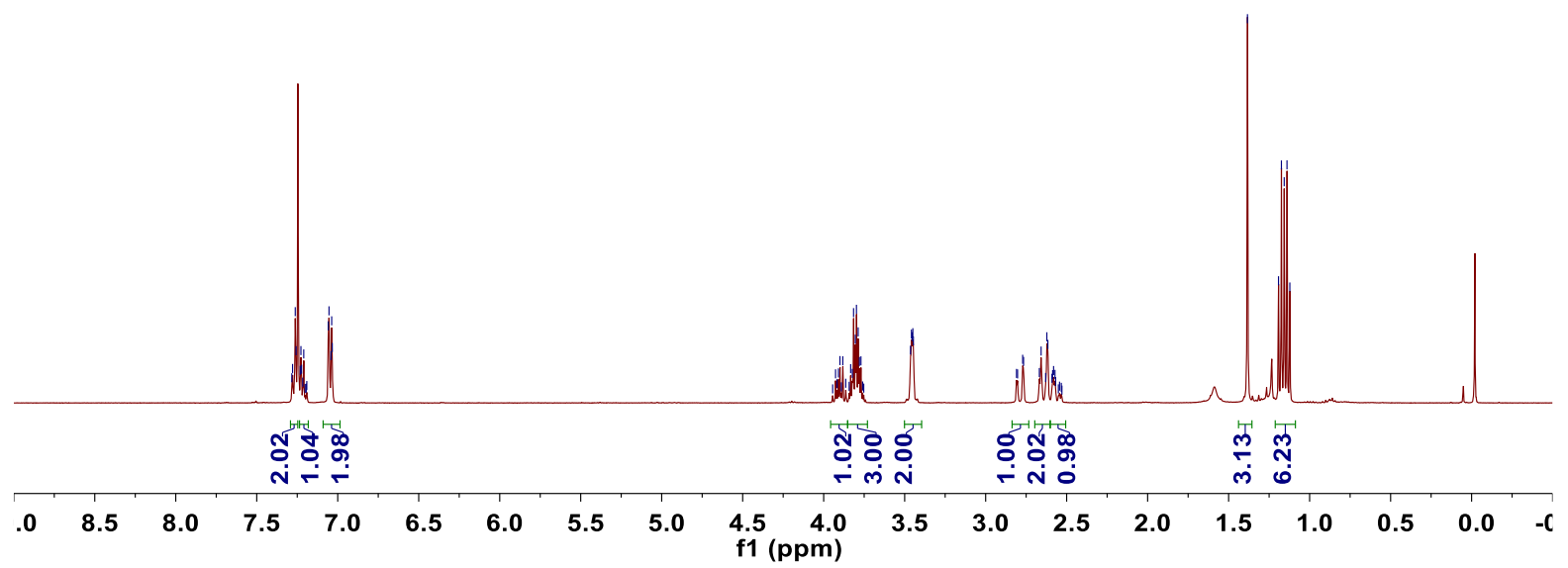

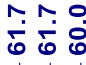

Vi

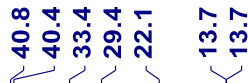

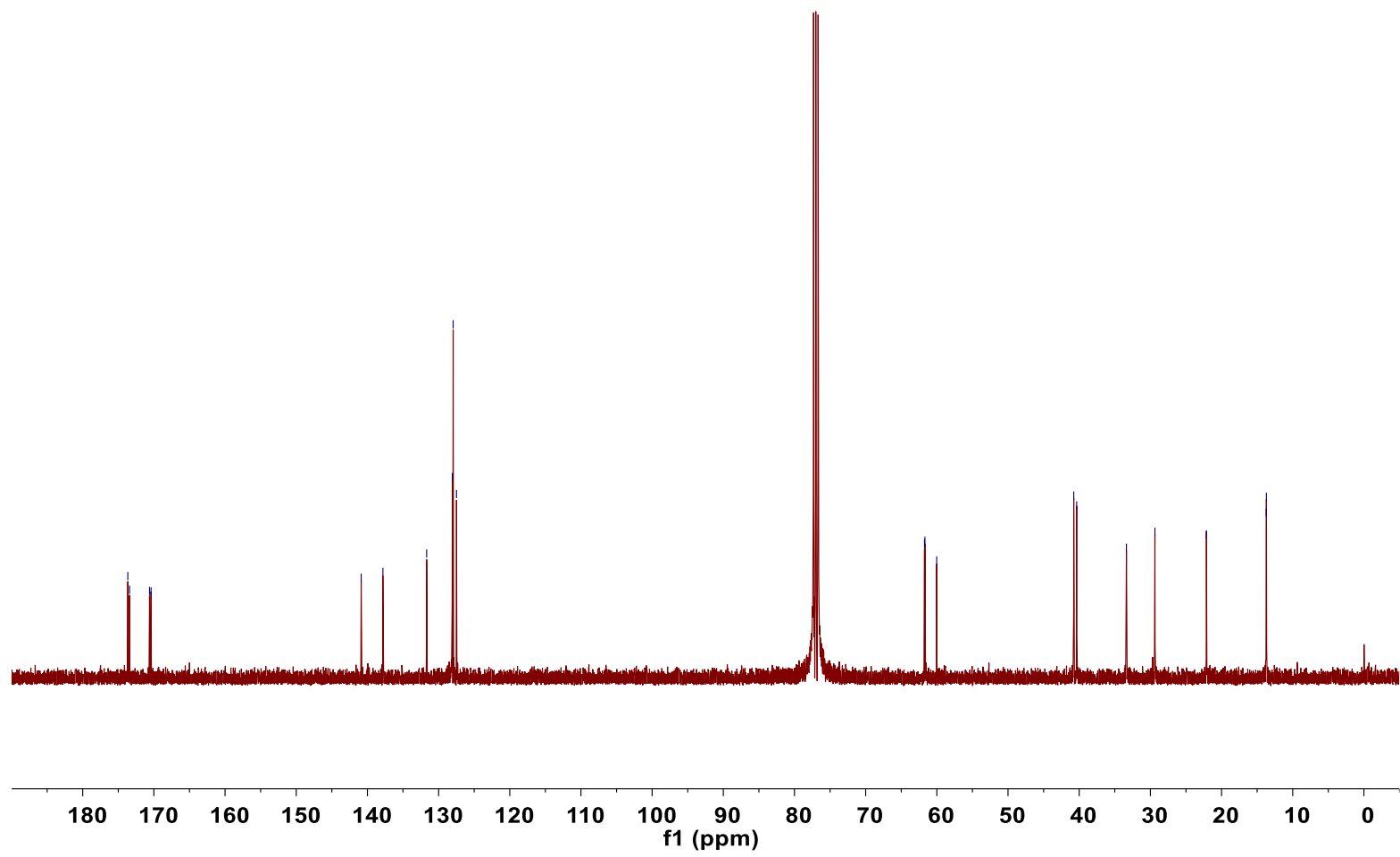

${ }^{1} \mathrm{H}$ and ${ }^{13} \mathrm{C}$ NMR spectra for product $5\left(400 \mathrm{MHz}, \mathrm{CDCl}_{3}\right)$ 

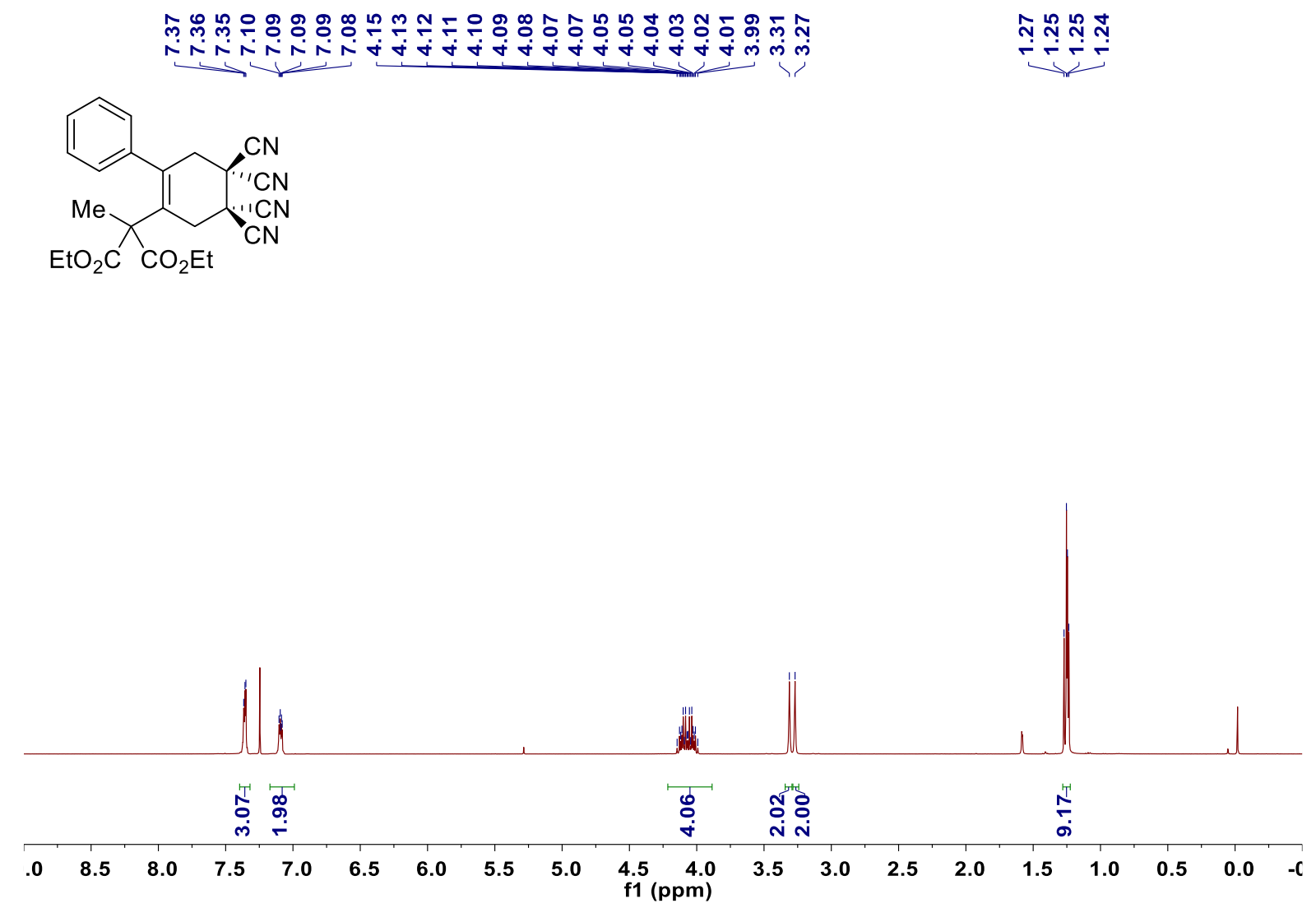

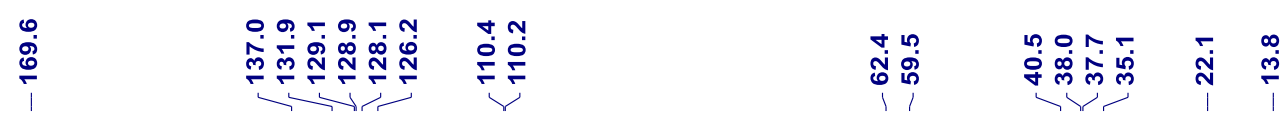

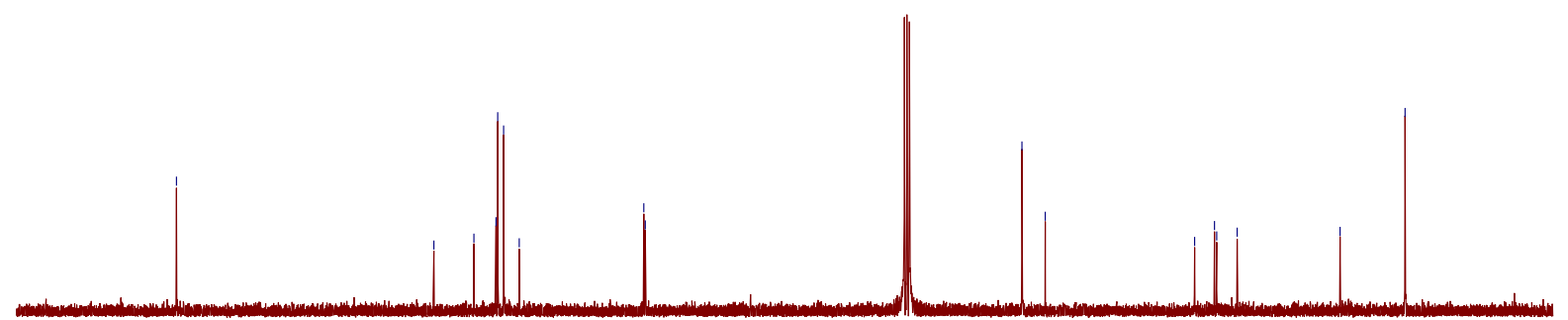

$\begin{array}{llllllllll}180 & 170 & 160 & 150 & 140 & 130 & 120 & 110 & \begin{array}{l}100 \\ \mathrm{f} 1(\mathrm{ppm})\end{array}\end{array}$

$\begin{array}{lllllllll}80 & 70 & 60 & 50 & 40 & 30 & 20 & 10 & 0\end{array}$

${ }^{1} \mathrm{H}$ and ${ }^{13} \mathrm{C}$ NMR spectra for product $6\left(400 \mathrm{MHz}, \mathrm{CDCl}_{3}\right)$ 


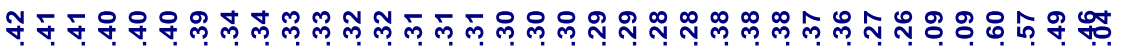

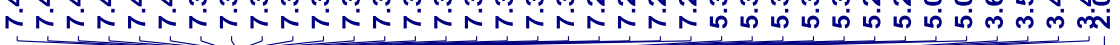<smiles>C=C(C(=C)C(C)(CO)CO)c1ccccc1</smiles>
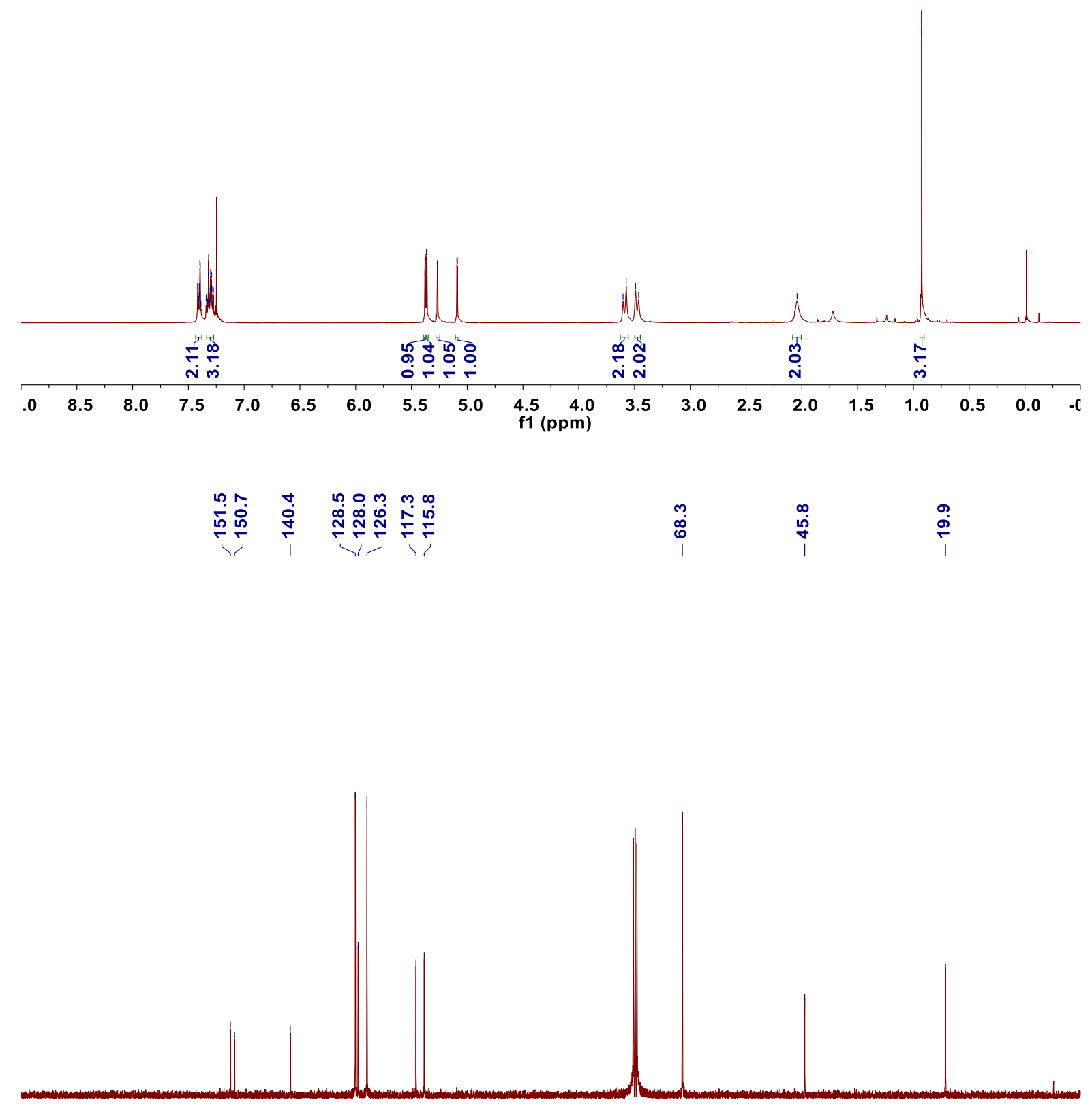

$\begin{array}{lllllllllll}90 & 180 & 170 & 160 & 150 & 140 & 130 & 120 & 110 & 100 & 90 \\ \mathrm{f} 1(\mathrm{ppm})\end{array}$

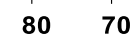

6050

$40 \quad 30$

$2010 \quad 0$

${ }^{1} \mathrm{H}$ and ${ }^{13} \mathrm{C}$ NMR spectra for product $7\left(400 \mathrm{MHz}, \mathrm{CDCl}_{3}\right)$ 

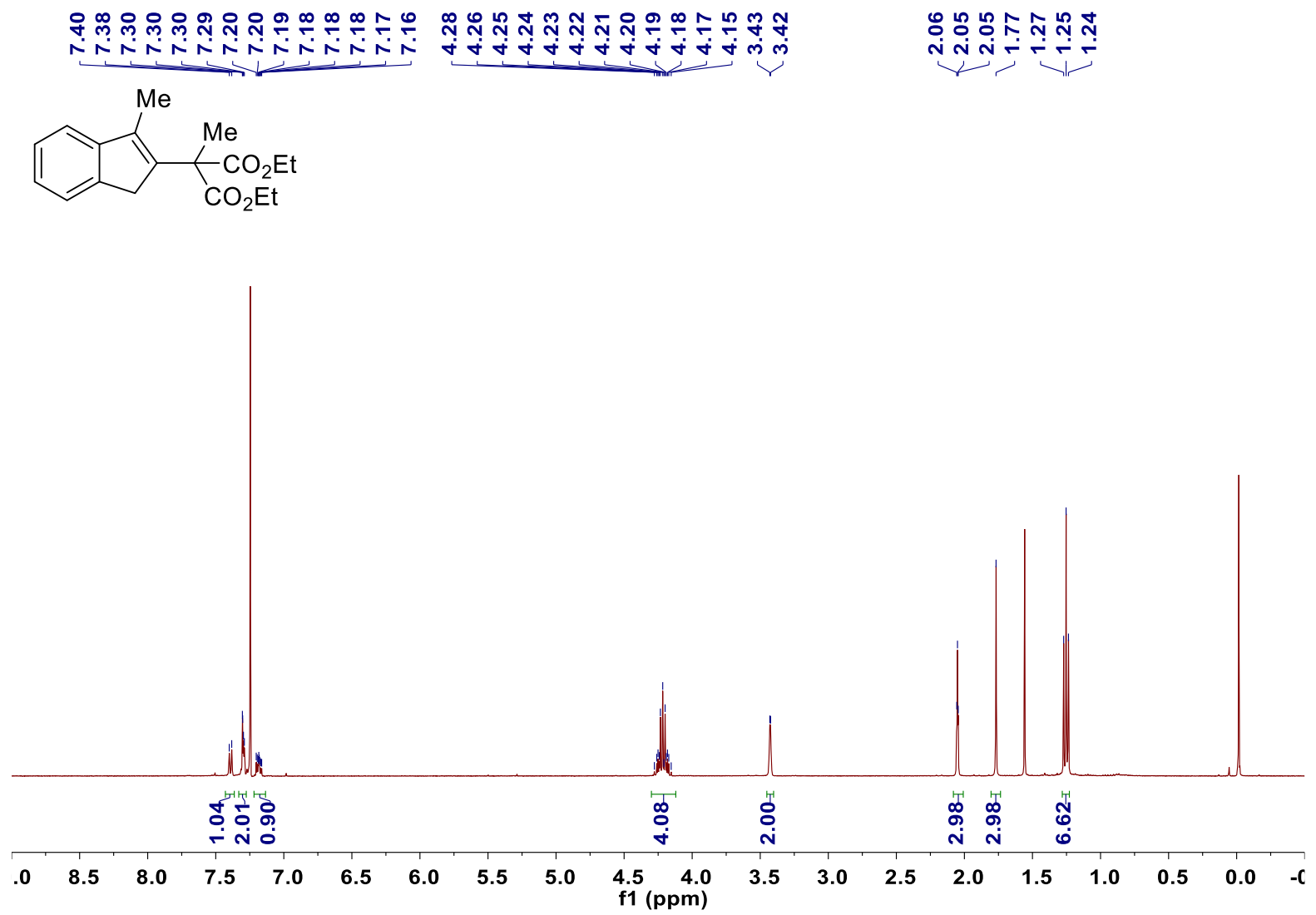

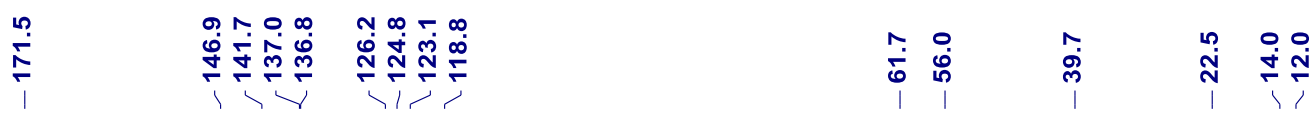

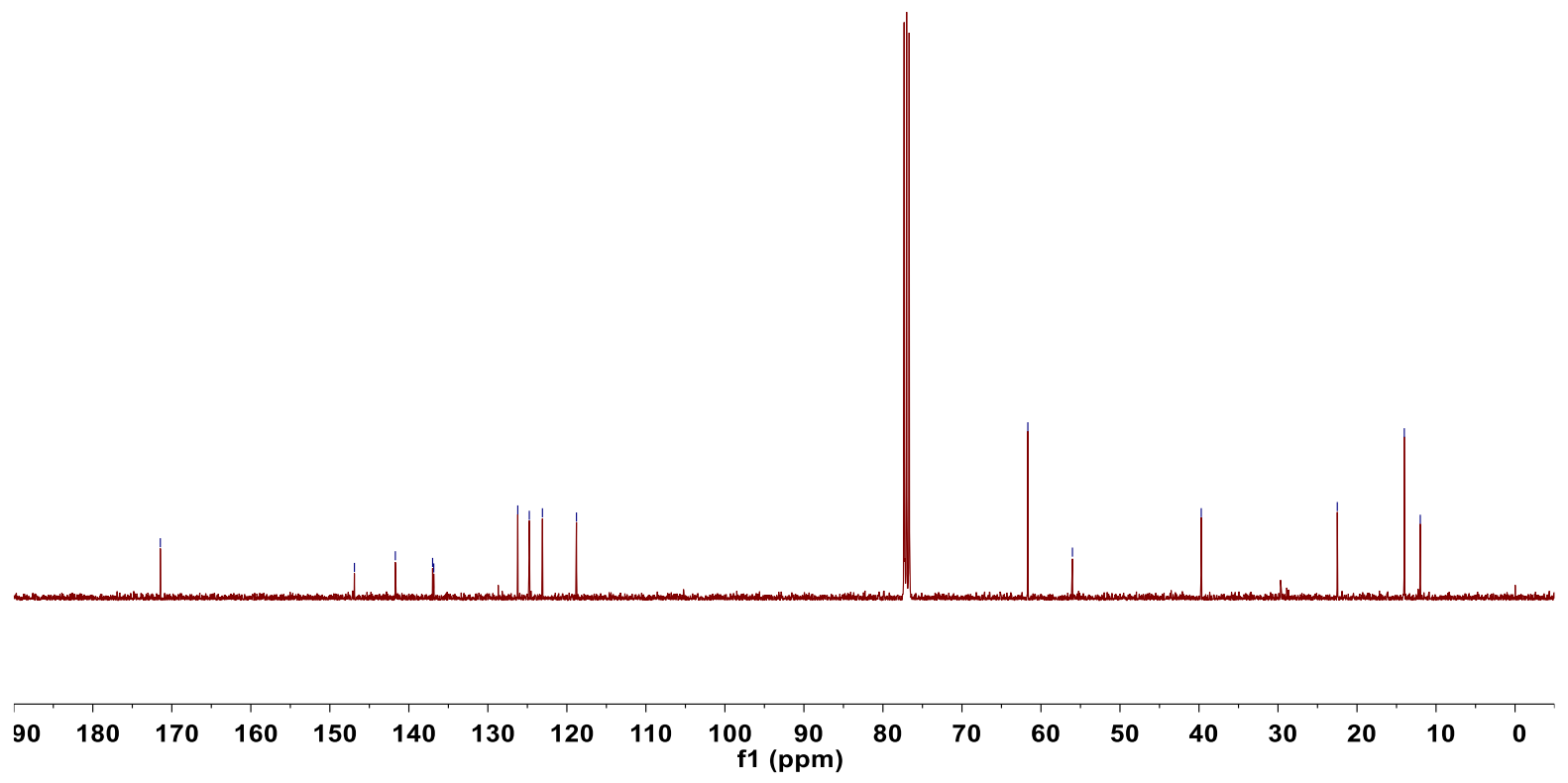

${ }^{1} \mathrm{H}$ and ${ }^{13} \mathrm{C}$ NMR spectra for product $8\left(400 \mathrm{MHz}, \mathrm{CDCl}_{3}\right)$ 


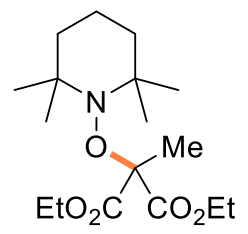

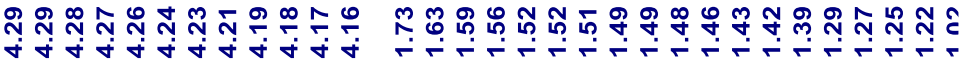

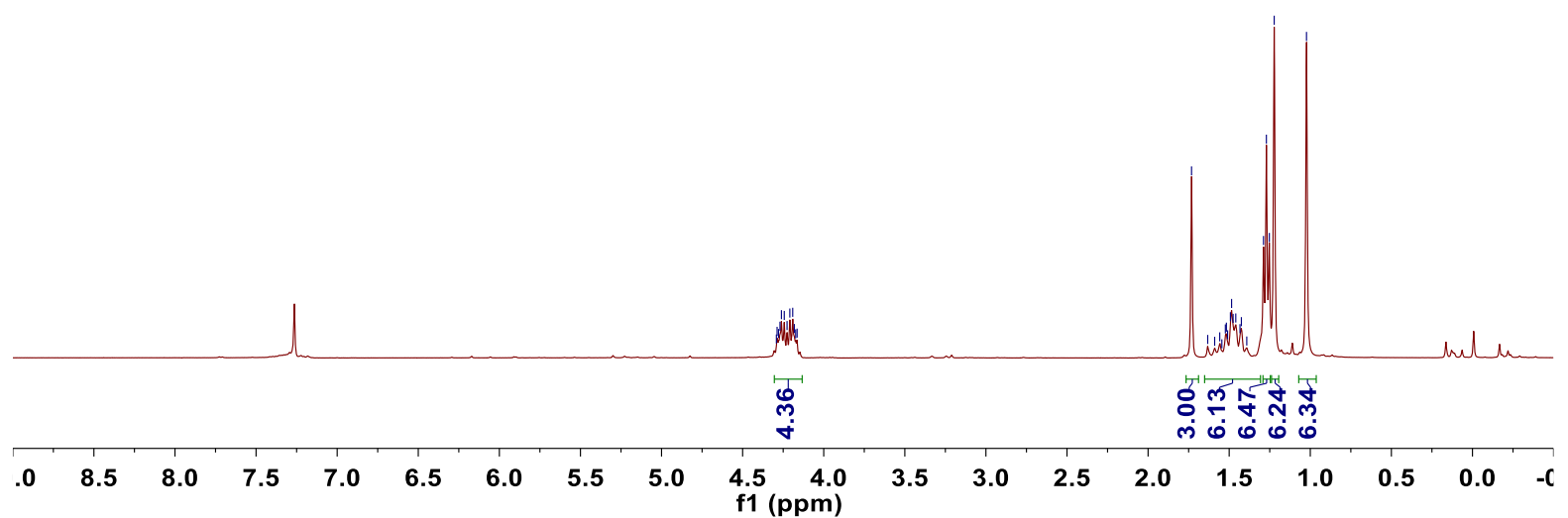

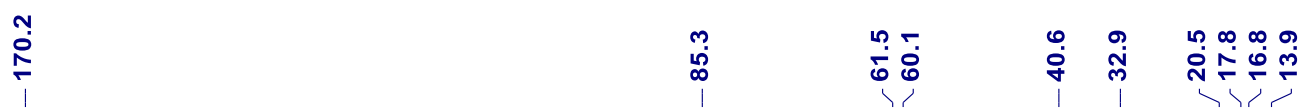

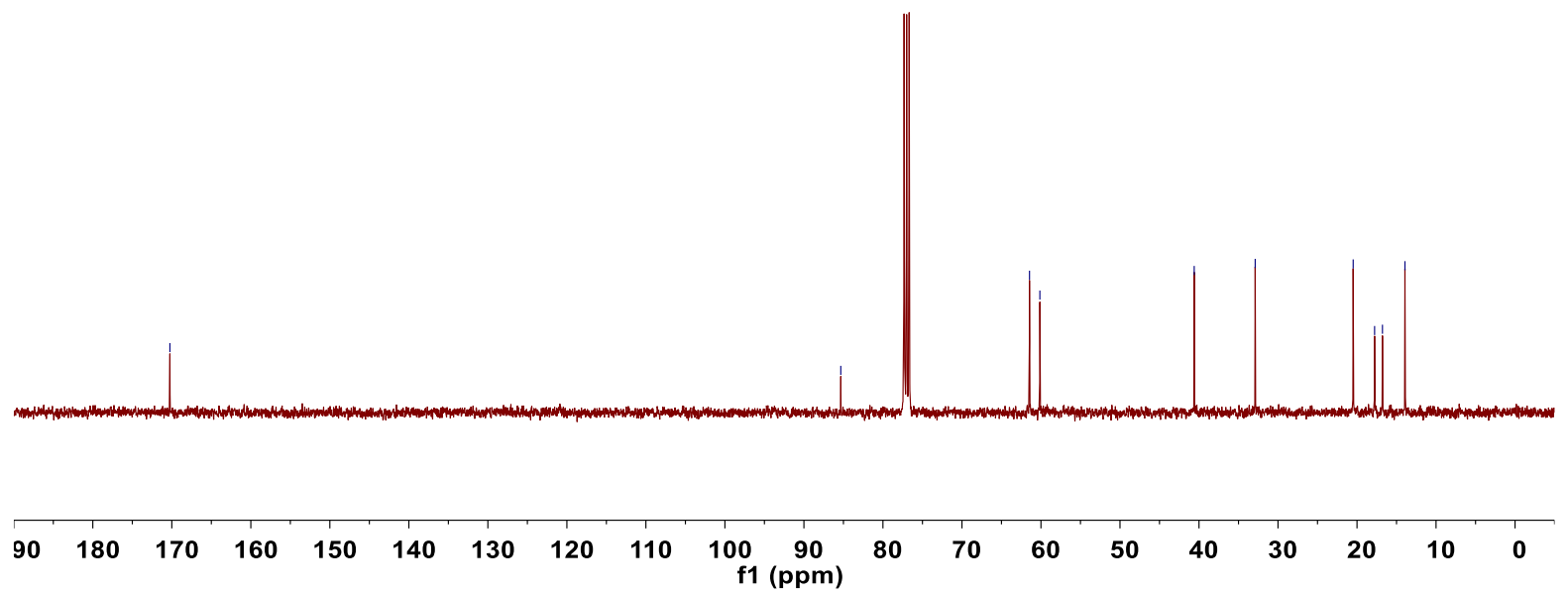

${ }^{1} \mathrm{H}$ and ${ }^{13} \mathrm{C}$ NMR spectra for product $9\left(400 \mathrm{MHz}, \mathrm{CDCl}_{3}\right)$ 